Supporting Information for

\title{
Radical Addition to Strained $\sigma$-bonds Enables the Stereocontrolled Synthesis of Cyclobutyl Boronic Esters
}

\author{
Mattia Silvi and Varinder K. Aggarwal \\ School of Chemistry, University of Bristol, Cantock's Close, \\ Bristol, BS8 1TS, United Kingdom
}

\section{Contents}

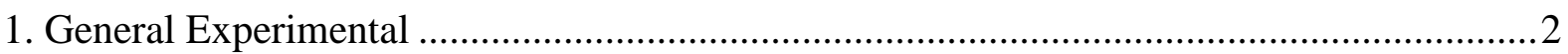

2. New Starting Materials Synthesis and Procedures …....................................................

3. Procedures \& Product Characterization ..................................................................... 7

3.1 General Procedure for the Preparation of Cyclobutyl Boronic Esters ........ 7

3.2 Reaction Products and Characterization …...................................... 8

3.3 General Procedure for the Reductive Desulfonylation ............................26

Product Functionalization Reactions............................................... 27

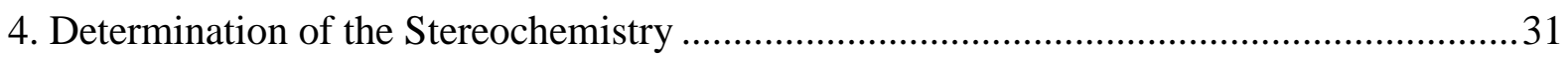

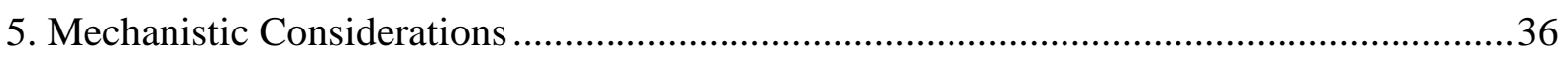

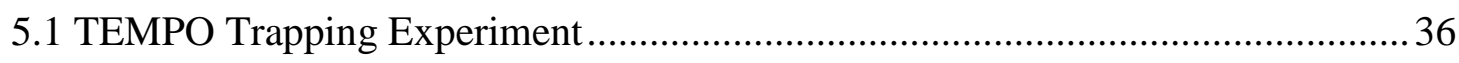

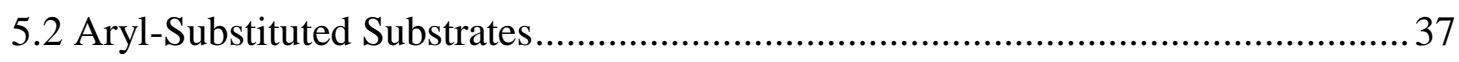

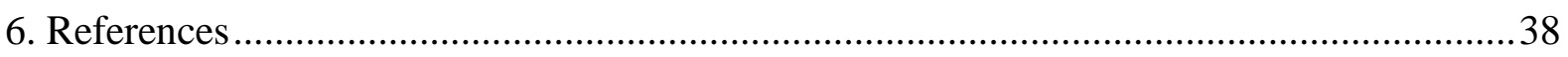

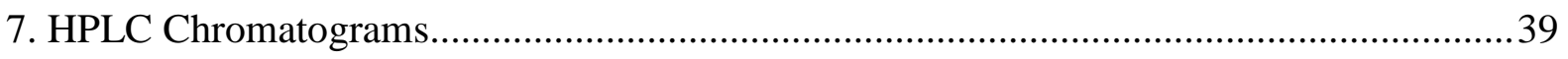

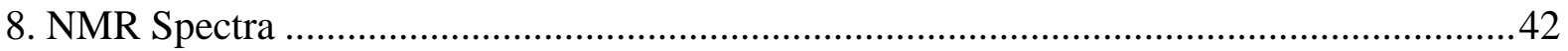




\section{General Experimental}

\section{Solvents, Reagents and Starting Materials}

All air and water-sensitive reactions were carried out in oven-dried glassware under a nitrogen atmosphere using standard Schlenk manifold technique. Bulk solutions were evaporated under reduced pressure using a Büchi rotary evaporator. All solvents were commercially supplied or provided by the communal stills of the School of Chemistry, University of Bristol. tert-BuLi was purchased from Sigma-Aldrich or Acros Organics. The molarity of organolithium solutions was determined by titration using $N$-benzyl benzamide as an indicator. Commercially available compounds were purchased from Sigma Aldrich, Alfa Aesar, Acros, Fluorochem or Santa Cruz Biotechnology and used as received. Iodosulfones 5t-5 $\mathbf{w}^{1}$ are known and were synthesized following procedures reported in the literature. Boronic esters $8 \mathbf{i},{ }^{2} \mathbf{8 n},{ }^{3} \mathbf{8 h},{ }^{4} \mathbf{8 m},{ }^{5}$ $\mathbf{8 0},{ }^{5} \mathbf{8 1},{ }^{6}$ are known and were synthesized according to literature procedures, $\mathbf{8 j},{ }^{5} \mathbf{8 k},{ }^{5,7}$ were also synthesised following literature procedures using (+)-sparteine. The synthesis of new compounds used as starting materials and their characterisation is detailed following. All other reagents were purchased from commercial sources and used as sold, unless noted.

\section{Chromatography and Instrumental Analysis}

Flash column chromatography (FCC) was carried out using Sigma-Aldrich silica gel LC60A$40(63 \mu \mathrm{m})$. All reactions were followed by thin-layer chromatography (TLC) when practical, using Merck Kieselgel $60 \mathrm{~F}_{254}$ fluorescent treated silica which was visualised under UV light or by staining with aqueous basic potassium permanganate.

${ }^{1} \mathbf{H}$ and ${ }^{13} \mathbf{C}$ NMR spectra were recorded using Jeol ECP (Eclipse) $300 \mathrm{MHz}$, Jeol ECS 400 MHz, Varian VNMR 400 MHz, Bruker 400 MHz, Varian VNMR 500 MHz and Bruker Cryo $500 \mathrm{MHz}$ spectrometers. Chemical shifts $(\delta)$ are given in parts per million (ppm), and coupling constants $(J)$ are given in Hertz $(\mathrm{Hz})$. The ${ }^{1} \mathrm{H}$ NMR spectra are reported as follows: ppm (multiplicity, coupling constants, number of protons). In most of the boronic esters ${ }^{13} \mathrm{C}$ NMR the carbon adjacent to the boron atom was not observed due to long relaxation time.

High resolution mass spectra (HRMS) were recorded on a Bruker Daltonics MicrOTOF II by Electrospray Ionisation (ESI). IR spectra were recorded on a Perkin Elmer Spectrum One FT-IR as a thin film. Only selected absorption maxima $\left(v_{\max }\right)$ are reported in wavenumbers $\left(\mathrm{cm}^{-1}\right)$. Melting points were recorded in degrees Celsius $\left({ }^{\circ} \mathrm{C}\right)$, using a Kofler hot-stage microscope apparatus and are reported uncorrected. 


\section{Naming of Compounds}

Compound names are those generated by ChemBioDraw 13.0 software (PerkinElmer), following the IUPAC nomenclature.

\section{New Starting Materials Synthesis and Procedures}

\section{Byciclobutyl-tolyl sulfoxide $(7)^{4}$}

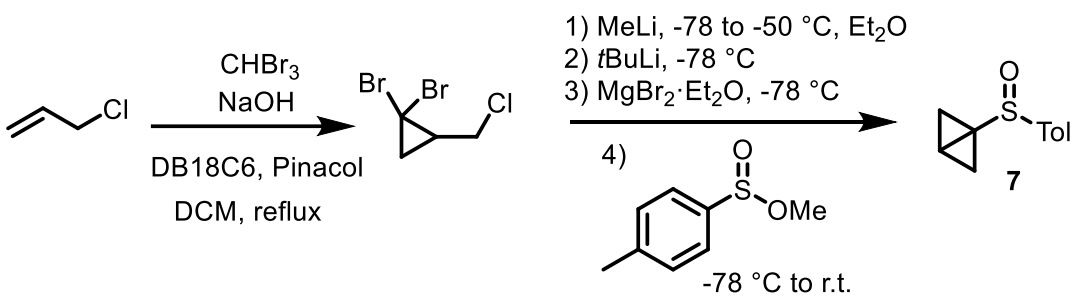

$250 \mathrm{~mL}$ flask was charged dibenzo-18-crown-6 (DB18C6) (2.14 g, $5.93 \mathrm{mmol}, 5 \mathrm{~mol} \%)$, pinacol (608 mg, $5.14 \mathrm{mmol}, 4 \mathrm{~mol} \%)$ under a $\mathrm{N}_{2}$ atmosphere. DCM $(75 \mathrm{~mL})$ was added to the solids followed by allyl chloride $(15.75 \mathrm{~mL}, 193 \mathrm{mmol}, 1.5$ equiv) and bromoform $(11.25 \mathrm{~mL}$, $129 \mathrm{mmol}, 1$ equiv). To the resulting solution, a solution of sodium hydroxide ( $48.9 \mathrm{~g}, 1.22$ mol, 9.5 equiv) in water ( $49 \mathrm{~mL})$ was added all at once. The flask was equipped with a reflux condenser and a $\mathrm{N}_{2}$ balloon and the reaction was stirred for $20 \mathrm{~h}$ at $40{ }^{\circ} \mathrm{C}$. After 20 hours GCMS of the crude reaction mixture revealed full conversion of bromoform (full consumption of bromoform is recommended, as the presence of this compound in the reaction mixture complicates the purification procedure).

The reaction mixture was allowed to cool down to r.t. and poured into a $500 \mathrm{~mL}$ beaker containing $250 \mathrm{~mL}$ of pentane. The mixture was gently stirred with a glass stick and sonicated for five minutes. The mixture was let to sediment for 20 minutes and the supernatant pentane was passed through a celite/silica plug, collecting the solution. Extra attention should be payed to avoid pouring the black precipitate or the water phase over the filter. The black precipitate was washed two times with $250 \mathrm{~mL}$ of pentane repeating the stirring-sonication-plug procedure.

The solvent was then evaporated, and the resulting residue was redissolved in $300 \mathrm{~mL}$ of pentane. The mixture was filtered through a thin layer of silica and the silica filter was washed with further $200 \mathrm{~mL}$ of pentane. Evaporation of solvent led to a turbid liquid, which was passed through a Pasteur containing a small amount of oven-dried $\mathrm{MgSO}_{4}$ to afford the pure 
intermediate trihalide as a colorless liquid (16.0 g, 51\% yield). ${ }^{1} \mathbf{H} \mathbf{~ N M R}\left(\mathrm{CDCl}_{3}, 400 \mathrm{MHz}\right) \delta$ (ppm): 3.65 (d, $J=7.4 \mathrm{~Hz}, 2 \mathrm{H}), 2.08-2.00$ (m, 1H), 1.93 (dd, $J=10.3,7.6 \mathrm{~Hz}, 1 \mathrm{H}), 1.48$ (app t, $J=7.5 \mathrm{~Hz}, 1 \mathrm{H}) ;{ }^{13} \mathbf{C ~ N M R}\left(\mathrm{CDCl}_{3}, 100 \mathrm{MHz}\right) \delta(\mathrm{ppm}): 46.3,32.3,29.1,25.8$. The spectral data were in accordance with the data reported in literature. ${ }^{4}$

Sulfoxide 7 was prepared from the intermediate trihalide following a literature procedure. ${ }^{4} \mathrm{~A}$ flame-dried nitrogen-flushed $500 \mathrm{~mL}$ three-necked flask was charged with 1,1-dibromo-2(chloromethyl)cyclopropane (3.85 g, $15.51 \mathrm{mmol}, 1.2$ equiv) and $\mathrm{Et}_{2} \mathrm{O}(80 \mathrm{~mL})$. The solution was cooled to $-78{ }^{\circ} \mathrm{C}$ (dry ice/acetone) then methyl lithium (in $\mathrm{Et}_{2} \mathrm{O}, 15.51 \mathrm{mmol}, 1.2$ equiv) was added at a rate of $0.6 \mathrm{~mL} / \mathrm{min}$ (syringe pump). The reaction was allowed to stir for $30 \mathrm{~min}$ at $-78{ }^{\circ} \mathrm{C}$ followed by $1 \mathrm{~h}$ at $-50{ }^{\circ} \mathrm{C}^{\mathrm{B}}$. The solution was then cooled back to $-78{ }^{\circ} \mathrm{C}$ (dry ice/acetone) and a high vacuum was applied for $2 \mathrm{~min}$ in order to remove the volatile $\mathrm{MeBr}$. tert-Butyl lithium (in pentane, $15.51 \mathrm{mmol}, 1.2$ equiv) ${ }^{\mathrm{C}}$ was then added at a rate of $0.6 \mathrm{~mL} / \mathrm{min}$ (syringe pump) and stirred for $20 \mathrm{~min}$. A solution of freshly prepared $\mathrm{MgBr}_{2} \bullet \mathrm{Et}_{2} \mathrm{O}^{\mathrm{D}}$ in a separate flask was then quickly added via a Teflon cannula ${ }^{\mathrm{E}}$. After stirring for $2 \mathrm{~h}$ at $-78{ }^{\circ} \mathrm{C}$, a solution of methyl 4-methylbenzenesulfinate ${ }^{4}(2.20 \mathrm{~g}, 12.92 \mathrm{mmol}, 1$ equiv) in THF (13 mL) was added at a rate of $3.5 \mathrm{~mL} / \mathrm{min}$ (syringe pump) and then the reaction mixture was stirred for 5 min at $-78{ }^{\circ} \mathrm{C}$ and finally for $30 \mathrm{~min}$ at ambient temperature. $\mathrm{NH}_{4} \mathrm{Cl}$ (saturated aqueous solution, $100 \mathrm{~mL}$ ) was added and the organic layer was collected. The aqueous phase was washed with EtOAc $(3 \times 100 \mathrm{~mL})$ and then the combined organic phases were dried $\left(\mathrm{MgSO}_{4}\right)$, filtered, and concentrated under reduced pressure. The crude residue was purified by flash column chromatography ( $\mathrm{SiO}_{2}$; gradient 40:60 - 60:40 EtOAc:pentane) to afford the BCBsulfoxide 7 (1.36 g, $55 \%)$ as a colorless oil which quickly solidifies to give a white - pale yellow crystalline solid ${ }^{\mathrm{F}} .{ }^{1} \mathbf{H}$ NMR $\left(\mathrm{CDCl}_{3}, 500 \mathrm{MHz}\right) \delta(\mathrm{ppm}): 7.56(\mathrm{~d}, J=8.1 \mathrm{~Hz}, 2 \mathrm{H}), 7.32$ $(\mathrm{d}, J=8.1 \mathrm{~Hz}, 2 \mathrm{H}), 2.42(\mathrm{~s}, 3 \mathrm{H}), 2.38-2.36(\mathrm{~m}, 1 \mathrm{H}), 2.11-2.08(\mathrm{~m}, 1 \mathrm{H}), 2.06-2.03(\mathrm{~m}$, 1H), $1.38-1.36(\mathrm{~m}, 1 \mathrm{H}), 1.21-1.19(\mathrm{~m}, 1 \mathrm{H}) .{ }^{13} \mathbf{C ~ N M R}\left(\mathrm{CDCl}_{3}, 126 \mathrm{MHz}\right) \delta(\mathrm{ppm}): 141.6$, $141.5,130.0,124.6,37.6,34.1,25.1,21.6,8.6$. The spectral data were in accordance with the data reported in literature. ${ }^{4} \mathrm{TLC}: \mathrm{R}_{\mathrm{f}}=0.24$ (60:40 EtOAc:pentane).

Notes: (A) Methyl lithium should be carefully titrated before use. (B) $\mathrm{A}-50{ }^{\circ} \mathrm{C}$ cooling bath is most conveniently prepared by adding chunks of dry ice to an acetone bath until the desired temperature is reached. The reaction is carefully, but quickly, transferred from the $-78{ }^{\circ} \mathrm{C}$ bath into the $-50{ }^{\circ} \mathrm{C}$ bath. (C) tert-Butyl lithium should be carefully titrated prior to use. (D) $\mathrm{MgBr}_{2} \bullet \mathrm{Et}_{2} \mathrm{O}$ was prepared as follows: A flame-dried nitrogen-flushed $250 \mathrm{~mL}$ flask, equipped 
with a reflux condenser, was charged with $\mathrm{Mg}$ turnings (3.06 g, $124 \mathrm{mmol}, 9.6$ equiv) and $\mathrm{Et}_{2} \mathrm{O}$ (40 mL). 1,2-dibromoethane (2.67 mL, $31.02 \mathrm{mmol}, 2.4$ equiv) was added dropwise until reflux was initiated. Reflux could be encouraged by gently heating up with a heating gun. The remaining 1,2-dibromoethane was added dropwise $(0.06 \mathrm{~mL} / \mathrm{min})$. After gas evolution had ceased, the mixture was stirred for additional $30 \mathrm{~min}$ at ambient temperature. (This should be prepared during the $1 \mathrm{~h}$ stir at $-50^{\circ} \mathrm{C}$.) (E) A Teflon cannula is not a necessity, but is the easiest to use. These can be prepared by cutting a piece of HPLC tubing to the required length and threading this through two appropriately sized suba-seals. We used BOLA PTFE tubing (ID $1.5 \mathrm{~mm}$, OD $2.5 \mathrm{~mm}$ ) purchased from Sigma-Aldrich. (F) The BCB-sulfoxide is slightly hygroscopic and should be stored in a freezer under an inert atmosphere.

\section{Methyl (2-iodoacetyl)-L-alanyl-L-phenylalaninate (5u)}

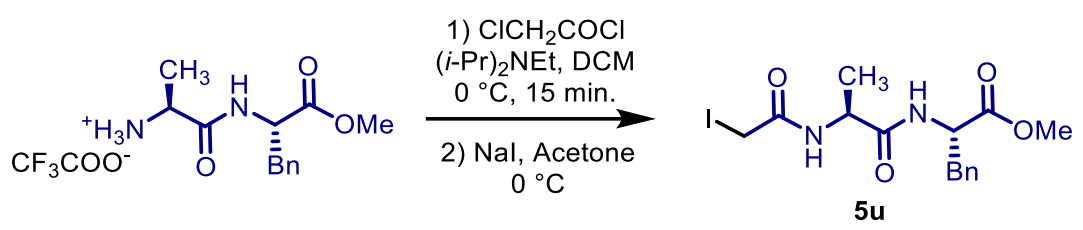

Protected dipeptide ${ }^{8}(213 \mathrm{mg}, 1.5 \mathrm{mmol})$ was suspended in $6 \mathrm{~mL}$ of dichloromethane and the temperature lowered down to $0{ }^{\circ} \mathrm{C} .288 \mu \mathrm{L}, 1.65 \mathrm{mmol}$ of Hünig's base were added, followed by dropwise addition of chloroacetyl chloride $(131 \mu \mathrm{L}, 1.65 \mathrm{mmol})$. The solution was stirred for 15 minutes at $0{ }^{\circ} \mathrm{C}$ and immediately quenched with the addition of $100 \mathrm{~mL}$ of $\mathrm{NH}_{4} \mathrm{Cl}$ sat. solution. The mixture was transferred in a separatory funnel, and the aqueous phase extracted twice with $50 \mathrm{~mL}$ of dichloromethane. The organic phase was collected and washed with brine $(50 \mathrm{~mL})$ and dried over magnesium sulfate. Solvents were removed under vacuo to afford a off-white solid.

The material was quickly purified by FCC (gradient hexanes:ethyl acetate 50:50 - 40:60) to afford $461 \mathrm{mg}$ of the intermediate chloride as a white solid.

The white solid was solubilised in $10 \mathrm{~mL}$ of acetone, placed in an ice bath, and sodium iodide (255 g, $1.7 \mathrm{mmol}$ ) was added to the solution. The solution was then allowed to stir at room temperature for 6 hours protected from light exposure. The solid was filtered out, and the solution was diluted with ethyl acetate $(100 \mathrm{~mL})$, transferred into a separatory funnel, and washed with sodium thiosulfate $10 \%$ solution $(50 \mathrm{~mL})$ and brine $(50 \mathrm{~mL})$. Solvents were removed under vacuo to afford compound $5 \mathbf{u}$ as a white solid (564 mg, $1.35 \mathrm{mmol}$, 90\% yield). $[\alpha]_{\mathbf{D}}{ }^{23}=+33.3(\mathrm{c}=0.95)$ MP: $141-143{ }^{\circ} \mathrm{C}($ EtOAc $) ; \mathbf{R}_{\mathbf{f}}(30 / 70 n$-hexane/EtOAc): 0.5; IR (film) $v_{\max } / \mathrm{cm}^{-1}: 3285,3062,2930,1742,1642,1535,1214,1171,700 .{ }^{1} \mathbf{H} \mathbf{~ N M R}\left(\mathrm{CDCl}_{3}\right.$, 
$500 \mathrm{MHz}) \delta(\mathrm{ppm}): 7.31-7.19(\mathrm{~m}, 3 \mathrm{H}), 7.13-7.07(\mathrm{~m}, 2 \mathrm{H}), 6.97(\mathrm{~d}, J=7.6 \mathrm{~Hz}, 1 \mathrm{H}), 6.71(\mathrm{~d}$, $J=7.9 \mathrm{~Hz}, 1 \mathrm{H}), 4.84(\mathrm{dt}, J=7.9,6.2 \mathrm{~Hz}, 1 \mathrm{H}), 4.50$ (p, $J=7.6 \mathrm{~Hz}, 1 \mathrm{H}), 3.72$ (s, 3H), 3.65 (s, 2H), $3.14(\mathrm{dd}, J=13.9,6.2 \mathrm{~Hz}, 1 \mathrm{H}), 3.08$ (dd, $J=13.9,6.2 \mathrm{~Hz}, 1 \mathrm{H}), 1.34$ (d, J = 7.6 Hz, 3H); ${ }^{13} \mathrm{C} \mathbf{N M R}\left(\mathrm{CDCl}_{3}, 125 \mathrm{MHz}\right) \delta(\mathrm{ppm}): 171.7,171.7,167.2,135.7,129.3,128.8,127.3,53.5$, 52.6, 49.5, 37.9, 18.3, -1.0; HRMS (ESI) mass calculated for $[\mathrm{M}+\mathrm{H}]^{+}\left(\mathrm{C}_{15} \mathrm{H}_{19} \mathrm{~N}_{2} \mathrm{O}_{4}{ }^{+}\right)$requires $m / z 419.0466$, found $m / z 419.0462$.

(2aS,4S,5' R,6aS,8aS,9S,10R,11aS)-5',6a,8a,9-tetramethyldocosahydrospiro[naphtho[2',1 ':4,5]indeno[2,1-b]furan-10,2'-pyran]-4-yl 2-iodoacetate (5v)

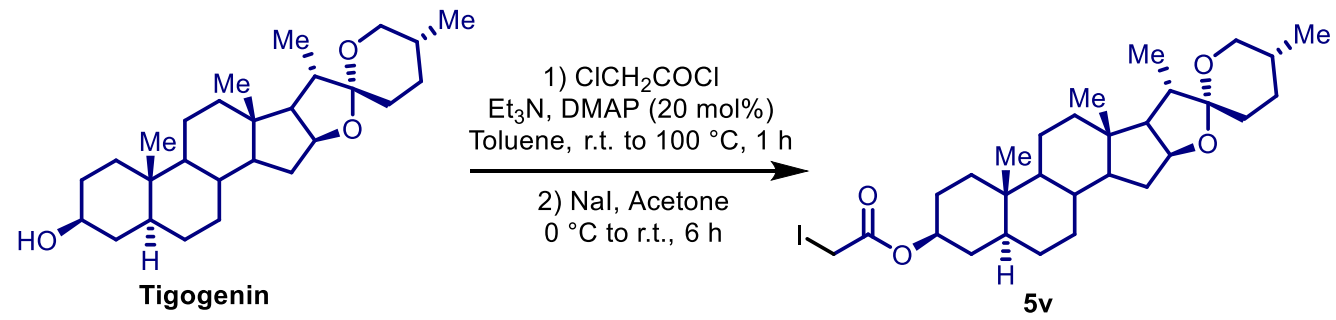

Tigogenin (645 mg, $1.5 \mathrm{mmol}$ ) was suspended in $20 \mathrm{~mL}$ of toluene, followed by the addition of 4-dimethylaminopyridine (36.6 mg, $0.03 \mathrm{mmol})$, triethylamine $(251 \mu \mathrm{L}, 1.8 \mathrm{mmol})$. To the solution, chloroacetyl chloride ( $143 \mu \mathrm{L}, 1.8 \mathrm{mmol}$ ) was added dropwise. The the reaction was equipped with a reflux condenser and heated at $100{ }^{\circ} \mathrm{C}$ for 1 hour, after which it was quenched with the addition of $100 \mathrm{~mL}$ of $\mathrm{NH}_{4} \mathrm{Cl}$ sat. solution. The mixture was transferred in a separatory funnel, and the aqueous phase extracted twice with $50 \mathrm{~mL}$ of ethyl acetate. The organic phase was collected and washed with brine $(50 \mathrm{~mL})$ and dried over magnesium sulfate. Solvents were removed under vacuo to afford a off-white solid that was purified by FCC (gradient hexane:ethyl acetate 100:0 - 95:5) to afford $581 \mathrm{mg}$ of intermediate chloride as a white solid. The powder was solubilised in $10 \mathrm{~mL}$ of acetone, placed in a ice bath, and sodium iodide (292 $\mathrm{mg}, 1.95 \mathrm{mmol}$ ) was added to the solution. The solution was then allowed to stir at room temperature for 6 hours protected from light exposure. The solid was filtered out, and the solution was diluted with ethyl acetate $(100 \mathrm{~mL})$, transferred into a separatory funnel, and washed with sodium thiosulfate $10 \%$ solution $(50 \mathrm{~mL})$ and brine $(50 \mathrm{~mL})$. Solvents were removed under vacuo to afford compound $\mathbf{5 v}$ as a white solid (634 $\mathrm{mg}, 1.08 \mathrm{mmol}, 72 \%$ yield). $[\alpha]_{\mathbf{D}^{24}}=-42.2(\mathrm{c}=0.95)$ MP: $162-164{ }^{\circ} \mathrm{C}($ EtOAc); Rf (30/70 $n$-hexane/EtOAc): 0.5; IR (film) $v_{\max } / \mathrm{cm}^{-1}: 2948,2870,1727,1451,1272,1052,1052,981,899,756 .{ }^{1} \mathbf{H} \mathbf{~ N M R}\left(\mathrm{CDCl}_{3}\right.$, $400 \mathrm{MHz}) \delta(\mathrm{ppm}): 4.71(\mathrm{tt}, J=11.1,5.0 \mathrm{~Hz}, 1 \mathrm{H}), 4.38(\mathrm{td}, J=7.7,6.3 \mathrm{~Hz}, 1 \mathrm{H}), 3.65(\mathrm{~s}, 2 \mathrm{H})$, $3.53-3.42(\mathrm{~m}, 1 \mathrm{H}), 3.36(\mathrm{t}, J=10.9 \mathrm{~Hz}, 1 \mathrm{H}), 2.02-1.92(\mathrm{~m}, 1 \mathrm{H}), 1.90-0.99(\mathrm{~m}, 24 \mathrm{H}), 0.95$ 
$(\mathrm{d}, J=7.0 \mathrm{~Hz}, 3 \mathrm{H}), 0.92-0.85(\mathrm{~m}, 1 \mathrm{H}), 0.84(\mathrm{~s}, 3 \mathrm{H}), 0.78(\mathrm{~d}, J=6.3 \mathrm{~Hz}, 3 \mathrm{H}), 0.76(\mathrm{~s}, 3 \mathrm{H})$, $0.70-0.61(\mathrm{~m}, 1 \mathrm{H}) ;{ }^{13} \mathbf{C ~ N M R}\left(\mathrm{CDCl}_{3}, 100 \mathrm{MHz}\right) \delta(\mathrm{ppm}): 168.6,109.4,80.9,75.6,67.0$, 62.3, 56.3, 54.3, 44.7, 41.7, 40.7, 40.1, 36.7, 35.7, 35.2, 33.6, 32.2, 31.9, 31.5, 30.4, 28.9, 28.6, 27.1, 21.1, 17.3, 16.6, 14.6, 12.4, -4.3; HRMS (ESI) mass calculated for $[\mathrm{M}+\mathrm{H}]^{+}$ $\left(\mathrm{C}_{15} \mathrm{H}_{19} \mathrm{~N}_{2} \mathrm{O}_{4}{ }^{+}\right)$requires $m / z$ 419.0466, found $\mathrm{m} / z 419.0462$.

\section{Procedures \& Product Characterization}

\subsection{General Procedure for the Preparation of Cyclobutyl Boronic Esters}

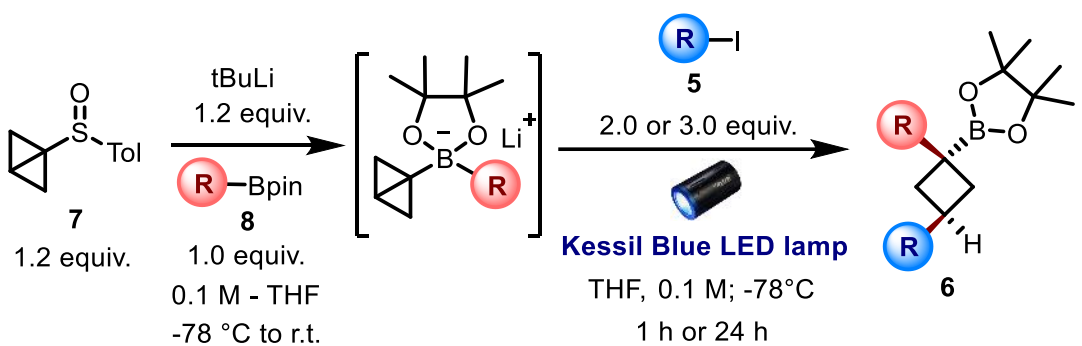

To a $-78{ }^{\circ} \mathrm{C}$ stirred solution of bicyclobutyl sulfoxide 7 (46.0 mg, $0.24 \mathrm{mmol}, 1.20$ equiv.) and boronic ester 8 (0.20 mmol, 1.0 equiv.) in THF (2.0 mL) under $\mathrm{N}_{2}$ atmosphere was added dropwise a pentane solution of tert-butyllithium ( $0.24 \mathrm{mmol}, 1.20$ equiv.). The solution was stirred for 5 minutes at $-78{ }^{\circ} \mathrm{C}$, and left to warm to r.t. under vigorous stirring over 15 minutes, after which it was cooled back to $-78{ }^{\circ} \mathrm{C}$ in a mirror-wall dewar using a cryostat. The alkyl iodide ( $0.40 \mathrm{mmol}, 2.0$ equiv. or $0.60 \mathrm{mmol}, 3.0$ equiv.) was quickly added to the top of the THF solution under a strong nitrogen flow and under irradiation. The vessel was sealed and the mixture was stirred vigorously for $1 \mathrm{~h}$ or $24 \mathrm{~h}$ at $-78{ }^{\circ} \mathrm{C}$ under a $\mathrm{N}_{2}$ atmosphere and constant irradiation.

After the stated time, the reaction was diluted with $10 \mathrm{~mL}$ of diethyl ether and filtered over a thin layer of silica. The solvents were removed under vacuo and the residue was purified by flash column chromatography to afford the desired cyclobutyl boronic esters 6. 


\section{Reaction set-up for irradiation of mixture with blue LEDs:}
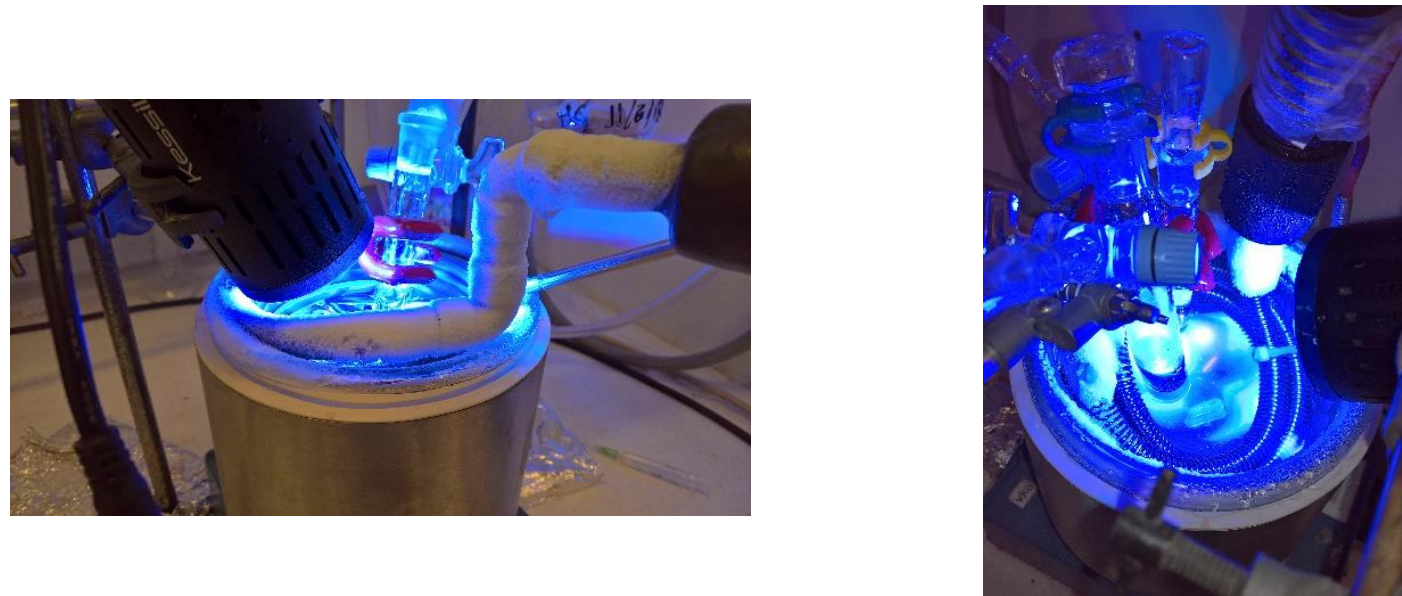

Figure S1. Reaction set-up. Light source: Kessil A160WE Tuna Blue.

\subsection{Reaction Products and Characterization}

2-(1-cyclohexyl-3-(trifluoromethyl)cyclobutyl)-4,4,5,5-tetramethyl-1,3,2-dioxaborolane (6a)

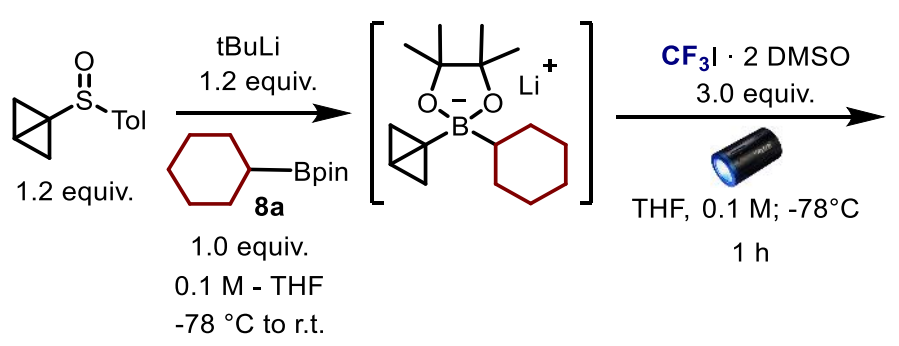

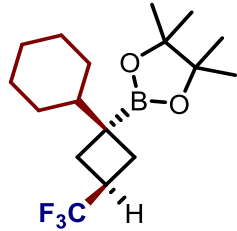

$6 \mathbf{a}$

According to the General Procedure, using cyclohexyl boronic acid pinacol ester 8a (44 $\mu \mathrm{L}$, $0.20 \mathrm{mmol}, 1.0$ equiv.) and trifluoromethyl iodide DMSO complex $(211 \mathrm{mg}, 138 \mu \mathrm{L}, 0.60$ mmol, 3.0 equiv.). The crude d.r. was measured to be $>20: 1$ by ${ }^{19} \mathrm{~F}-\mathrm{NMR}$ analysis. The product was purified by FCC (gradient hexane/dichloromethane $=100: 0-90: 10$ ) to afford the title compound $6 \mathbf{a}$ as a white solid in 74\% yield (49 $\mathrm{mg})$; d.r. of the purified product $>20: 1$.

MP: $76-77^{\circ} \mathrm{C}$ (hexane); $\mathbf{R}_{\mathbf{f}}\left(70 / 30 n\right.$-hexane/dichloromethane): 0.5; IR (film) $v_{\max } / \mathrm{cm}^{-1}: 2979$, 2924, 2852, 1378, 1276, 1140, 1089, 967, 838, 668; ${ }^{\mathbf{1}} \mathbf{H} \mathbf{N M R}\left(\mathrm{CDCl}_{3}, 400 \mathrm{MHz}\right) \delta(\mathrm{ppm}): \delta$ $2.84-2.65(\mathrm{~m}, 1 \mathrm{H}), 2.27-2.12(\mathrm{~m}, 2 \mathrm{H}), 1.92-1.80(\mathrm{~m}, 2 \mathrm{H}), 1.75-1.57(\mathrm{~m}, 5 \mathrm{H}), 1.32-1.20$ $(\mathrm{m}, 13 \mathrm{H}), 1.20-1.04(\mathrm{~m}, 3 \mathrm{H}), 1.01-0.87(\mathrm{~m}, 2 \mathrm{H}) .{ }^{13} \mathbf{C ~ N M R}\left(\mathrm{CDCl}_{3}, 125 \mathrm{MHz}\right) \delta(\mathrm{ppm})$ : $127.1(\mathrm{q}, J=126.4 \mathrm{~Hz}), 83.4,48.3,33.0(\mathrm{q}, J=31.0 \mathrm{~Hz}), 28.8(\mathrm{q}, J=3.2 \mathrm{~Hz}), 28.7,26.8(\mathrm{x} 2)$, 24.9. ${ }^{19} \mathbf{F ~ N M R}\left(\mathrm{CDCl}_{3}, 377 \mathrm{MHz}\right) \delta(\mathrm{ppm}):-73.40(\mathrm{~d}, J=9.0 \mathrm{~Hz})$. HRMS (MALDI) mass calculated for $[\mathrm{M}+\mathrm{Na}]^{+}\left(\mathrm{C}_{17} \mathrm{H}_{28} \mathrm{O}_{2} \mathrm{BF}_{3} \mathrm{Na}^{+}\right)$requires $m / z$ 355.2030, found $m / z$ 355.2039. 
4,4,5,5-tetramethyl-2-(1-methyl-3-(trifluoromethyl)cyclobutyl)-1,3,2-dioxaborolane (6b)
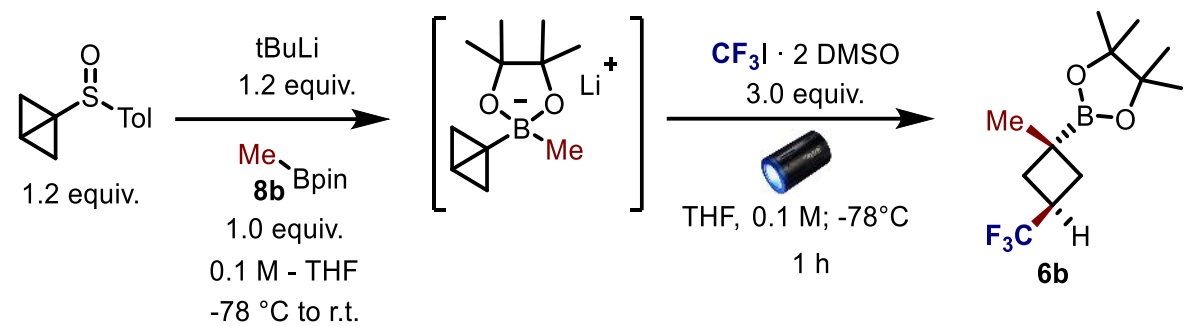

According to the General Procedure, using methyl boronic acid pinacol ester 8b $(33 \mu \mathrm{L}$, $0.20 \mathrm{mmol}, 1.0$ equiv.) and trifluoromethyl iodide DMSO complex $(211 \mathrm{mg}, 138 \mu \mathrm{L}, 0.60$ mmol, 3.0 equiv.). The crude d.r. was measured to be $11.1: 1$ by ${ }^{19} \mathrm{~F}-\mathrm{NMR}$ analysis. The product was purified by FCC (gradient pentane/dichloromethane $=100: 0-90: 10$ ) to afford the title compound $\mathbf{6 b}$ as a colourless oil in $66 \%$ yield $(35 \mathrm{mg}$ ); d.r. of the purified product $>20: 1$.

$\mathbf{R}_{\mathbf{f}}\left(80 / 20\right.$ pentane/dichloromethane): 0.6; IR (film) $v_{\max } / \mathrm{cm}^{-1}: 2980,2936,1372,1314,1277$, 1206, 1137, 1079, 967, 838, 667; ${ }^{1} \mathbf{H}$ NMR $\left(\mathrm{CDCl}_{3}, 500 \mathrm{MHz}\right) \delta(\mathrm{ppm}): 2.95-2.80(\mathrm{~m}, 1 \mathrm{H})$, $2.31-2.21(\mathrm{~m}, 2 \mathrm{H}), 1.86-1.75(\mathrm{~m}, 2 \mathrm{H}), 1.26(\mathrm{~s}, 12 \mathrm{H}), 1.14(\mathrm{~s}, 3 \mathrm{H}) ;{ }^{13} \mathbf{C} \mathbf{~ N M R}\left(\mathrm{CDCl}_{3}\right.$, $125 \mathrm{MHz}) \delta(\mathrm{ppm}): 127.1$ (q, $J=274.6 \mathrm{~Hz}), 83.6,32.9(\mathrm{q}, J=31.0 \mathrm{~Hz}), 31.4(\mathrm{q}, J=3.3 \mathrm{~Hz})$, 24.9, 24.8; ${ }^{19}$ F NMR $\left(\mathrm{CDCl}_{3}, 377 \mathrm{MHz}\right) \delta(\mathrm{ppm})$ : $-73.60(\mathrm{~d}, J=9.1 \mathrm{~Hz})$. HRMS (EI) mass calculated for $\left[\mathrm{M}-\mathrm{CH}_{3}\right]^{+}\left(\mathrm{C}_{12} \mathrm{H}_{21} \mathrm{O}_{3} \mathrm{BF}_{3}{ }^{+}\right)$requires $m / z, 291.1738$, found $m / z, 291.1738$.

\section{4,4,5,5-tetramethyl-2-(1-phenethyl-3-(trifluoromethyl)cyclobutyl)-1,3,2-dioxaborolane} $(6 c)$

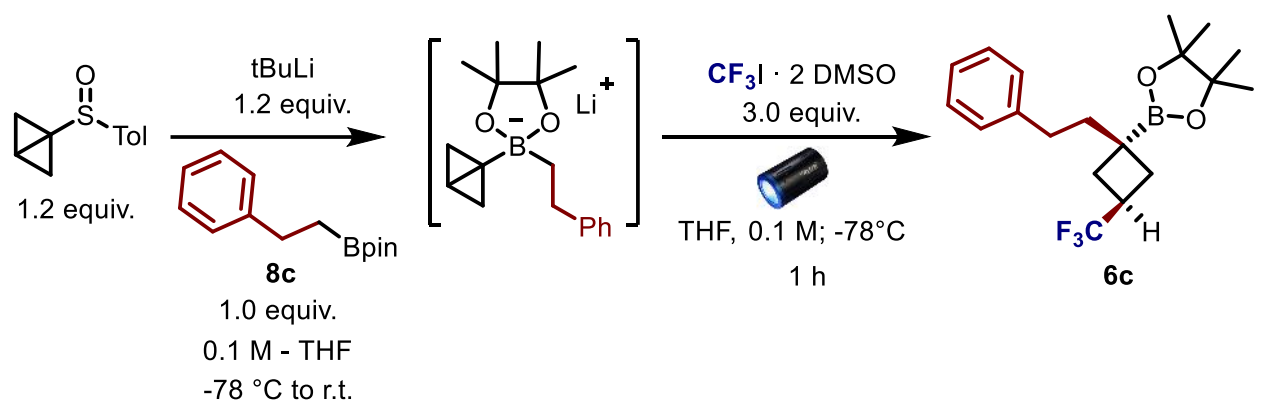

According to the General Procedure, using phenethyl boronic acid pinacol ester 8c $(46.4 \mathrm{mg}$, $0.20 \mathrm{mmol}, 1.0$ equiv.) and trifluoromethyl iodide DMSO complex $(211 \mathrm{mg}, 138 \mu \mathrm{L}, 0.60$ mmol, 3.0 equiv.). The crude d.r. was measured to be $13.6: 1$ by ${ }^{19} \mathrm{~F}-\mathrm{NMR}$ analysis. The product was purified by FCC (gradient hexane/dichloromethane $=100: 0-80: 20$ ) to afford the title compound $\mathbf{6 c}$ as a colourless oil in $73 \%$ yield (52 mg); d.r. of the purified product $>20: 1$.

$\mathbf{R}_{\mathbf{f}}$ (70/30 $n$-hexane/dichloromethane): 0.5; IR (film) $v_{\max } / \mathrm{cm}^{-1}: 2978,2939,1372,1316,1277$, 1208, 1139, 1082, 697; ${ }^{\mathbf{1}} \mathbf{H}$ NMR $\left(\mathrm{CDCl}_{3}, 400 \mathrm{MHz}\right) \delta(\mathrm{ppm}): 7.29-7.23$ (m, 2H), 7.20 - 7.12 $(\mathrm{m}, 3 \mathrm{H}), 2.99-2.81(\mathrm{~m}, \mathrm{~J}=9.1 \mathrm{~Hz}, 1 \mathrm{H}), 2.52-2.42(\mathrm{~m}, 2 \mathrm{H}), 2.30-2.20(\mathrm{~m}, 2 \mathrm{H}), 1.90-1.77$ 
$(\mathrm{m}, 4 \mathrm{H}), 1.28(\mathrm{~s}, 12 \mathrm{H}) \cdot{ }^{13} \mathbf{C} \mathbf{N M R}\left(\mathrm{CDCl}_{3}, 100 \mathrm{MHz}\right) \delta(\mathrm{ppm}): 142.6,128.4,127.1$ (q, $J=126.4$ $\mathrm{Hz}), 125.8,83.7,42.0,33.3(\mathrm{q}, J=31.0 \mathrm{~Hz}), 32.9,29.9$ (q, $J=3.2 \mathrm{~Hz}), 24.9 .{ }^{19} \mathbf{F ~ N M R}\left(\mathrm{CDCl}_{3}\right.$, $377 \mathrm{MHz}) \delta(\mathrm{ppm}):-73.53(\mathrm{~d}, J=8.9 \mathrm{~Hz})$. HRMS (EI) mass calculated for $[\mathrm{M}+\mathrm{Na}]^{+}$ $\left(\mathrm{C}_{19} \mathrm{H}_{26} \mathrm{BO}_{2} \mathrm{~F}_{3}{ }^{+}\right)$requires $\mathrm{m} / \mathrm{z} 354.1972$, found $\mathrm{m} / \mathrm{z} 354.1968$.

\section{2-(1-(tert-butyl)-3-(trifluoromethyl)cyclobutyl)-4,4,5,5-tetramethyl-1,3,2-dioxaborolane}

(6d)

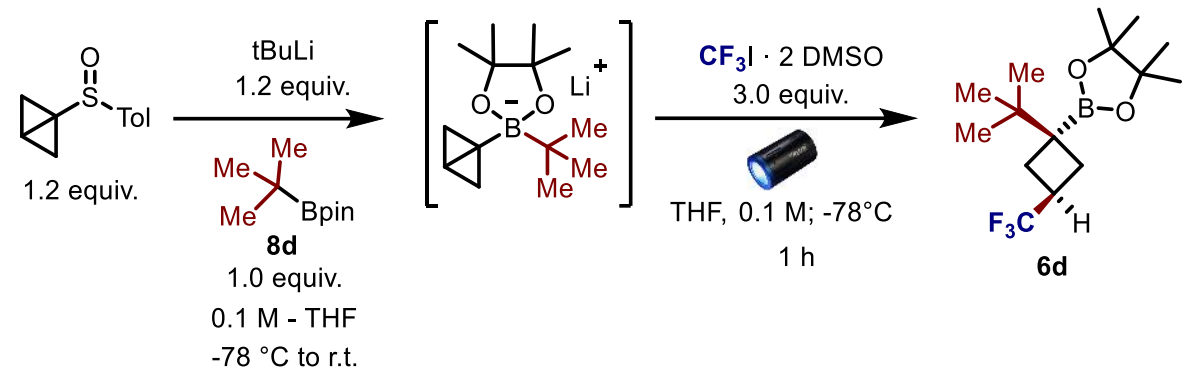

According to the General Procedure, using tert-butyl boronic acid pinacol ester $8 \mathbf{d}(44 \mu \mathrm{L}$, $0.20 \mathrm{mmol}, 1.0$ equiv.) and trifluoromethyl iodide DMSO complex $(211 \mathrm{mg}, 138 \mu \mathrm{L}, 0.60$ mmol, 3.0 equiv.). The crude d.r. was measured to be $>20: 1$ by ${ }^{19} \mathrm{~F}-\mathrm{NMR}$ analysis, although ${ }^{11} \mathrm{~B}-\mathrm{NMR}$ shown the formation of a 2:1 mixture boronic ester/borinic ester (35 ppm; $50 \mathrm{ppm}$ ). The mixture was warmed to $0{ }^{\circ} \mathrm{C}$ and $2 \mathrm{~mL}$ of methanol were added, followed by the addition of urea-hydrogen peroxide complex $(75 \mathrm{mg}, 0.8 \mathrm{mmol})$. The reaction was warmed to r.t. and stirred for $1 \mathrm{~h}$ after which it was quenched with the addition of $100 \mathrm{~mL}$ of $10 \% \mathrm{w} / \mathrm{w}$ sodium thiosulfate solution, and extracted with pentane $(3 \times 100 \mathrm{~mL})$. The organic phase was washed with brine and dried over $\mathrm{MgSO}_{4}$. Solvents were evaporated and the product was purified by FCC (gradient pentane/dichloromethane $=100: 0-90: 10$ ) to afford the title compound $6 \mathbf{d}$ as a colourless oil in $33 \%$ yield $(20 \mathrm{mg})$; d.r. of the purified product $>20: 1$.

$\mathbf{R}_{\mathbf{f}}\left(80 / 20\right.$ pentane/dichloromethane): 0.60; IR (film) $v_{\max } / \mathrm{cm}^{-1}: 2973,1368,1353,1192,1146$, 1123, 1042, 967, 839, 661; ${ }^{1} \mathbf{H}$ NMR $\left(\mathrm{CDCl}_{3}, 500 \mathrm{MHz}\right) \delta(\mathrm{ppm}): 2.70$ - $2.54(\mathrm{~m}, 1 \mathrm{H}), 2.14-$ $1.98(\mathrm{~m}, 4 \mathrm{H}), 1.27(\mathrm{~s}, 12 \mathrm{H}), 0.88(\mathrm{~s}, 9 \mathrm{H}) ;{ }^{13} \mathbf{C ~ N M R}\left(\mathrm{CDCl}_{3}, 125 \mathrm{MHz}\right) \delta(\mathrm{ppm}): 127.3$ (q, $J=$ $126.4 \mathrm{~Hz}), 83.5,33.1$ (q, $J=31.0 \mathrm{~Hz}), 31.9,29.9,26.3,25.1(\mathrm{q}, J=3.2 \mathrm{~Hz}), 24.9 ;{ }^{19} \mathbf{F ~ N M R}$ $\left(\mathrm{CDCl}_{3}, 377 \mathrm{MHz}\right) \delta(\mathrm{ppm}):-73.15(\mathrm{~d}, J=9.0 \mathrm{~Hz})$. HRMS (ESI) mass calculated for $[\mathrm{M}+\mathrm{H}]^{+}$ $\left(\mathrm{C}_{19} \mathrm{H}_{23} \mathrm{O}_{3}{ }^{+}\right)$requires $m / z$ 299.1642, found $m / z$ 299.1645. 
4,4,5,5-tetramethyl-2-(1-phenyl-3-(trifluoromethyl)cyclobutyl)-1,3,2-dioxaborolane (6e)

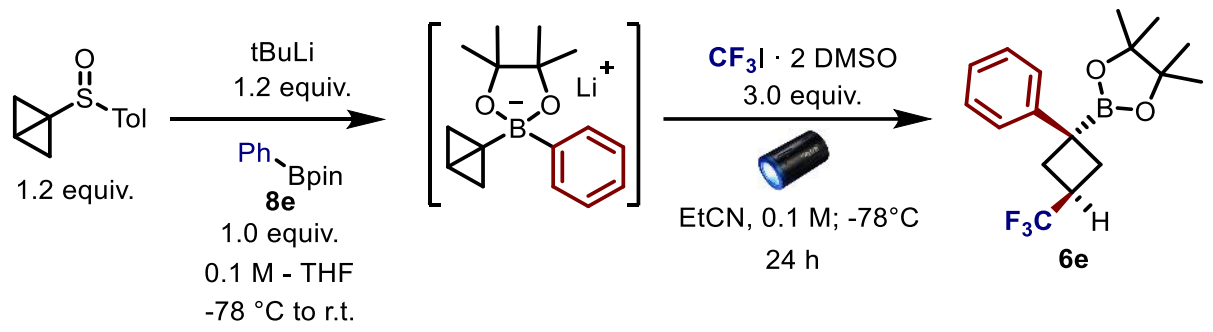

Following a modification of the General Procedure, using phenyl boronic acid pinacol ester $8 \mathbf{e}$ (40.8 mg, $0.20 \mathrm{mmol}, 1.0$ equiv.) and trifluoromethyl iodide DMSO complex (211 mg, $138 \mu \mathrm{L}$, $0.60 \mathrm{mmol}, 3.0$ equiv.). After boronate complex formation, the solvent was removed under vacuum, followed by the addition of dry proprionitrile. The reaction was cooled down to $-78^{\circ} \mathrm{C}$ and trifluoromethyl iodide DMSO complex was added on the surface of the solution under a nitrogen flow and under irradiation. The reaction was stirred under irradiation at $-78{ }^{\circ} \mathrm{C}$ in propionitrile for 24 hours. The crude d.r. was measured to be $4.5: 1$ by ${ }^{19} \mathrm{~F}-\mathrm{NMR}$ analysis. The reaction was worked up following the General Procedure and purified by FCC (gradient hexane/ethyl acetate $=100: 0-95: 5)$ to afford the title compound $\mathbf{6 e}$ as a white solid in $75 \%$ yield (49 mg); d.r. of the purified product 4.5:1.

MP: $61-63{ }^{\circ} \mathrm{C}$ ( $n$-hexane/ethyl acetate); $\mathbf{R} \mathbf{f}\left(85 / 15 n\right.$-hexane/EtOAc): 0.6; IR (film) $v_{\max } / \mathrm{cm}^{-1}$ : 2979, 1354, 1319, 1144, 1093, 837, 698; ${ }^{1} \mathbf{H}$ NMR (Major diastereoisomer, $\mathrm{CDCl}_{3}, 400 \mathrm{MHz}$ ) $\delta$ (ppm): 7.25 - $7.15(\mathrm{~m}, 2 \mathrm{H}), 7.13-6.96(\mathrm{~m}, 3 \mathrm{H}), 3.01-2.84(\mathrm{~m}, 1 \mathrm{H}), 2.66-2.55(\mathrm{~m}, 2 \mathrm{H})$, $2.37-2.23(\mathrm{~m}, 2 \mathrm{H}), 1.12(\mathrm{~s}, 12 \mathrm{H}) ;{ }^{13} \mathbf{C ~ N M R}$ (Major diastereoisomer, $\mathrm{CDCl}_{3}, 100 \mathrm{MHz}$ ) $\delta$ (ppm): 147.2, 128.3, 126.7 (q, $J=274.6 \mathrm{~Hz}), 125.4,125.2,84.1,33.9$ (q, $J=31.5 \mathrm{~Hz}), 31.0$ (q, $J=3.3 \mathrm{~Hz}$ ), 24.6; ${ }^{19} \mathbf{F}$ NMR (Major diastereoisomer, $\left.\mathrm{CDCl}_{3}, 377 \mathrm{MHz}\right) \delta(\mathrm{ppm}):-73.3(\mathrm{~d}$, $\mathrm{J}=8.7 \mathrm{~Hz})$. HRMS $(\mathrm{ESI})$ mass calculated for $[\mathrm{M}+\mathrm{H}]^{+}\left(\mathrm{C}_{17} \mathrm{H}_{22} \mathrm{BF}_{3} \mathrm{O}_{2}{ }^{+}\right)$requires $\mathrm{m} / \mathrm{z}$ 327.1738, found $m / z 327.1748$.

\section{2-(1-(4-methoxyphenyl)-3-(trifluoromethyl)cyclobutyl)-4,4,5,5-tetramethyl-1,3,2-dioxab orolane (6f)}

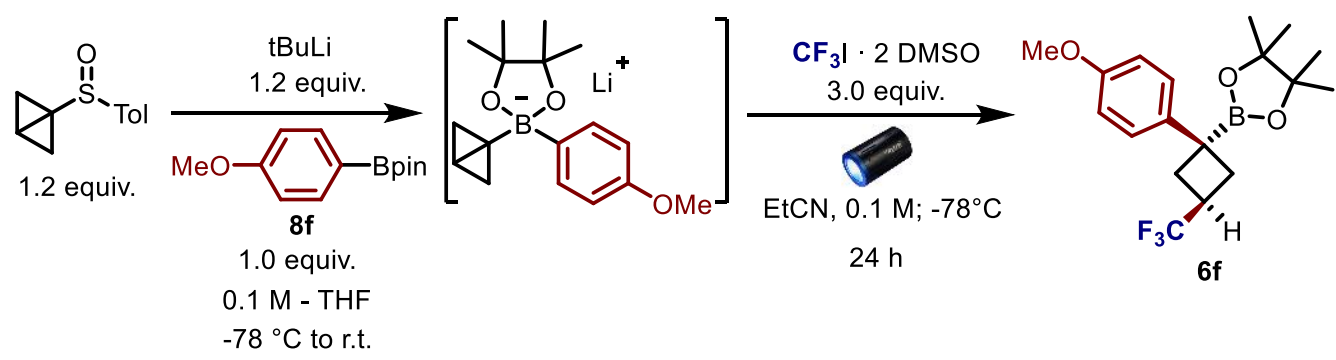

Following a modification of the General Procedure, using 4-methoxyphenyl boronic acid pinacol ester $\mathbf{8 f}$ (46.8 mg, $0.20 \mathrm{mmol}, 1.0$ equiv.) and trifluoromethyl iodide DMSO complex 
(211 mg, $138 \mu \mathrm{L}, 0.60 \mathrm{mmol}, 3.0$ equiv.). After boronate complex formation, the solvent was removed under vacuum, followed by the addition of dry proprionitrile. The reaction was cooled down to $-78{ }^{\circ} \mathrm{C}$ and trifluoromethyl iodide DMSO complex was added on the surface of the solution under a nitrogen flow and under irradiation. The reaction was stirred under irradiation at $-78{ }^{\circ} \mathrm{C}$ in propionitrile for 24 hours. The crude d.r. was measured to be 5.8:1 by ${ }^{19} \mathrm{~F}-\mathrm{NMR}$ analysis. The reaction was worked up following the General Procedure and purified by FCC (gradient hexane/dichloromethane $=70: 30-60: 40$ ) to afford the title compound $\mathbf{6 f}$ as a colourless oil in $62 \%$ yield (44 mg); d.r. of the purified product $>20: 1$.

$\mathbf{R}_{\mathbf{f}}$ (50/50 $n$-hexane/EtOAc): 0.7; IR (film) $v_{\max } / \mathrm{cm}^{-1}: 2978,1600,1510,1250,1145,829,673$; ${ }^{1} \mathbf{H}$ NMR $\left(\mathrm{CDCl}_{3}, 500 \mathrm{MHz}\right) \delta(\mathrm{ppm}): 7.05$ - 6.94 (m, 2H), 6.87 - 6.77 (m, 2H), $3.78(\mathrm{~s}, 3 \mathrm{H})$, $3.07-2.83(\mathrm{~m}, 1 \mathrm{H}), 2.72-2.54(\mathrm{~m}, 2 \mathrm{H}), 2.39-2.24(\mathrm{~m}, 2 \mathrm{H}), 1.20(\mathrm{~s}, 12 \mathrm{H}) ;{ }^{13} \mathbf{C ~ N M R}\left(\mathrm{CDCl}_{3}\right.$, $125 \mathrm{MHz}) \delta(\mathrm{ppm}): \delta 157.3,139.2,126.8(\mathrm{q}, J=277.0 \mathrm{~Hz}), 126.4,113.8,84.0,55.3,33.7(\mathrm{q}$, $J=32.8 \mathrm{~Hz}), 31.1(\mathrm{q}, J=3.3 \mathrm{~Hz}), 29.9,24.6 . ;{ }^{19} \mathbf{F} \mathbf{N M R}\left(\mathrm{CDCl}_{3}, 470 \mathrm{MHz}\right) \delta(\mathrm{ppm}):-73.33$ $(\mathrm{d}, \mathrm{J}=8.6 \mathrm{~Hz})$. HRMS (ESI) mass calculated for $\left[\mathrm{M}+\mathrm{NH}_{4}\right]^{+}\left(\mathrm{C}_{18} \mathrm{H}_{28} \mathrm{BF}_{3} \mathrm{NO}_{3}{ }^{+}\right)$requires $\mathrm{m} / z$ 374.2112 , found $\mathrm{m} / \mathrm{z} 374.2110$.

\section{2-(1-(4-chlorophenyl)-3-(trifluoromethyl)cyclobutyl)-4,4,5,5-tetramethyl-1,3,2-dioxabor olane (6g)}
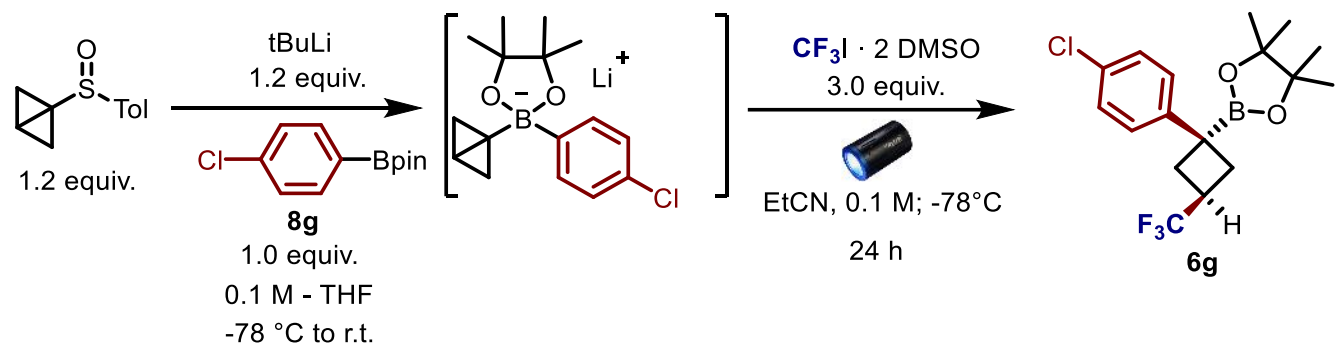

Following a modification of the General Procedure, using 4-chlorophenyl boronic acid pinacol ester $8 \mathrm{~g}$ (47.6 mg, $0.20 \mathrm{mmol}, 1.0$ equiv.) and trifluoromethyl iodide DMSO complex (211 mg, $138 \mu \mathrm{L}, 0.60 \mathrm{mmol}, 3.0$ equiv.). After boronate complex formation, the solvent was removed under vacuum, followed by the addition of dry proprionitrile. The reaction was cooled down to $-78^{\circ} \mathrm{C}$ and trifluoromethyl iodide DMSO complex was added on the surface of the solution under a nitrogen flow and under irradiation. The reaction was stirred under irradiation at $-78{ }^{\circ} \mathrm{C}$ in propionitrile for 24 hours. The crude d.r. was measured to be $2: 1$ by ${ }^{19} \mathrm{~F}-\mathrm{NMR}$ analysis. The reaction was worked up following the General Procedure and purified by FCC (gradient hexane/dichloromethane $=80: 20-70: 30$ ) to afford the title compound $\mathbf{6 g}$ as a white solid in $51 \%$ yield (37 mg); d.r. of the purified product $>20: 1$. 
MP: $112-113{ }^{\circ} \mathrm{C}$ ( $n$-hexane/EtOAc); $\mathbf{R}_{\mathbf{f}}\left(50 / 50 n\right.$-hexane/EtOAc): $0.65 ; \mathbf{I R}$ (film) $v_{\max } / \mathrm{cm}^{-1}$ : 2981, 1350, 1323, 1144, 1126, 1087, 841, 755; ${ }^{1} \mathbf{H} \mathbf{~ N M R}\left(\mathrm{CDCl}_{3}, 400 \mathrm{MHz}\right) \delta$ (ppm): $7.26-$ $7.22(\mathrm{~m}, 2 \mathrm{H}), 7.02-6.97(\mathrm{~m}, 2 \mathrm{H}), 3.10-2.87(\mathrm{~m}, 1 \mathrm{H}), 2.74-2.57(\mathrm{~m}, 2 \mathrm{H}), 2.41-2.19(\mathrm{~m}$, 2H), 1.19 (s, 12H); ${ }^{13} \mathbf{C ~ N M R}\left(\mathrm{CDCl}_{3}, 100 \mathrm{MHz}\right) \delta(\mathrm{ppm}): 145.9,131.0,128.5,127.7,126.8$ $(\mathrm{q}, J=277.0 \mathrm{~Hz}), 84.2,33.7(\mathrm{q}, J=32.8 \mathrm{~Hz}), 31.1(\mathrm{q}, J=3.3 \mathrm{~Hz}), 24.6 ;{ }^{19} \mathbf{F ~ N M R}\left(\mathrm{CDCl}_{3}\right.$, $377 \mathrm{MHz}) \delta(\mathrm{ppm}):-73.48(\mathrm{~d}, J=8.7 \mathrm{~Hz})$. HRMS (APCI) mass calculated for $[\mathrm{M}+\mathrm{H}]^{+}$ $\left(\mathrm{C}_{17} \mathrm{H}_{21} \mathrm{BClF}_{3} \mathrm{O}_{2}{ }^{+}\right)$requires $m / z$ 361.1348, found $m / z 361.1359$.

\section{4,4,5,5-tetramethyl-2-((1s,1'r,3S,3'R)-1'-methyl-3'-phenyl-3-(trifluoromethyl)-[1,1'-} bi(cyclobutan)]-1-yl)-1,3,2-dioxaborolane (6h)

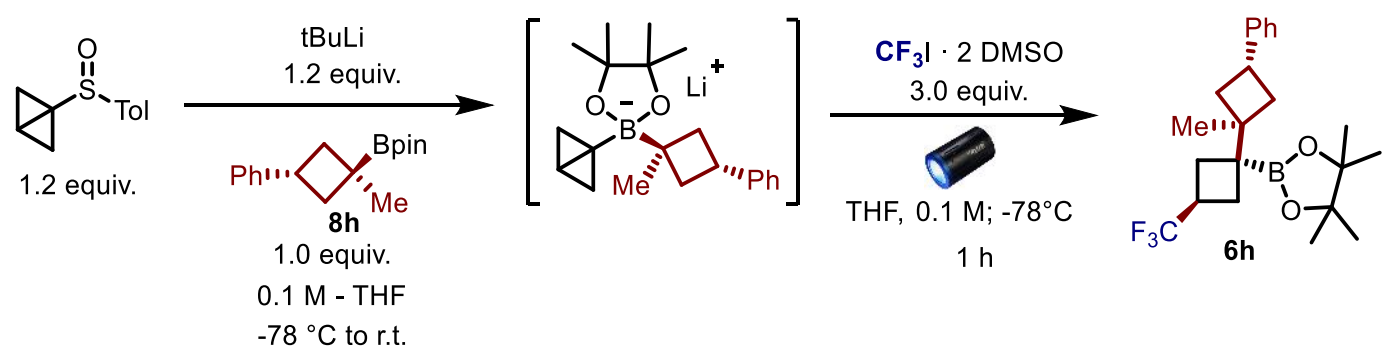

According to the General Procedure, using boronic ester $\mathbf{8 h}$ (54 mg, $0.20 \mathrm{mmol}, 1.0$ equiv.) and trifluoromethyl iodide DMSO complex (211 mg, $138 \mu \mathrm{L}, 0.60 \mathrm{mmol}, 3.0$ equiv.). The crude d.r. was measured to be $12.5: 1$ by ${ }^{19} \mathrm{~F}-\mathrm{NMR}$ analysis. The product was purified by FCC (gradient hexane/dichloromethane $=85: 15-80: 20$ ) to afford the title compound $\mathbf{6 h}$ as a white solid in $40 \%$ yield $(31 \mathrm{mg})$; d.r. of the purified product 12.5:1.

MP: 38 - $40{ }^{\circ} \mathrm{C}$ ( $n$-hexane); $\mathbf{R}_{\mathbf{f}}$ (70/30 $n$-hexane/EtOAc): 0.4; IR (film) $v_{\max } / \mathrm{cm}^{-1}: 2925,2855$, 1361, 1312, 1275, 1143, 1044, 750, 697; ${ }^{1} \mathbf{H}$ NMR $\left(\mathrm{CDCl}_{3}, 500 \mathrm{MHz}\right) \delta(\mathrm{ppm}): \delta$ 7.33-7.26 $(\mathrm{m}, 2 \mathrm{H}), 7.26-7.22(\mathrm{~m}, 2 \mathrm{H}), 7.19-7.14(\mathrm{~m}, 1 \mathrm{H}), 3.28(\mathrm{p}, J=9.0 \mathrm{~Hz}, 1 \mathrm{H}), 2.92-2.79(\mathrm{~m}, 1 \mathrm{H})$, $2.74-2.64(\mathrm{~m}, 2 \mathrm{H}), 2.23-2.11(\mathrm{~m}, 4 \mathrm{H}), 1.94-1.87(\mathrm{~m}, 2 \mathrm{H}), 1.27(\mathrm{~s}, 12 \mathrm{H}), 1.02(\mathrm{~s}, 3 \mathrm{H})$; ${ }^{13} \mathrm{C} \mathrm{NMR}\left(\mathrm{CDCl}_{3}, 125 \mathrm{MHz}\right) \delta(\mathrm{ppm}): 147.2,128.3,127.4(\mathrm{q}, J=262.3 \mathrm{~Hz}), 126.6,125.6$, 83.5, 37.9, 37.5, 32.87, 32.5 (q, $J=30.5 \mathrm{~Hz}), 27.0,25.8$ (q, $J=3.1 \mathrm{~Hz}), 24.8 ;{ }^{19} \mathbf{F} \mathbf{N M R}\left(\mathrm{CDCl}_{3}\right.$, $376 \mathrm{MHz}) \delta(\mathrm{ppm}):-72.78(\mathrm{~d}, J=8.9 \mathrm{~Hz})$. HRMS (ESI) mass calculated for $[\mathrm{M}+\mathrm{H}]^{+}$ $\left(\mathrm{C}_{22} \mathrm{H}_{30} \mathrm{~F}_{3} \mathrm{O}_{2} \mathrm{~B}^{+}\right)$requires $\mathrm{m} / z$ 395.2364, found $\mathrm{m} / \mathrm{z}$ 395.2375. 
4,4,5,5-tetramethyl-2-(1-((R)-1-phenylethyl)-3-(trifluoromethyl)cyclobutyl)-1,3,2dioxaborolane (6i)
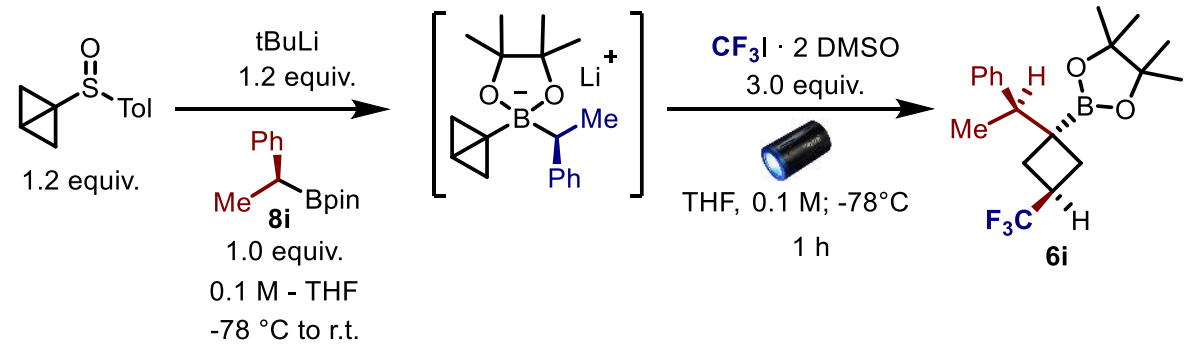

According to the General Procedure, using boronic ester $8 \mathbf{i}$ (46.4 mg, $0.20 \mathrm{mmol}, 1.0$ equiv.) and trifluoromethyl iodide DMSO complex (211 mg, $138 \mu \mathrm{L}, 0.60 \mathrm{mmol}, 3.0$ equiv.). The crude d.r. was measured to be $20: 1$ by ${ }^{19} \mathrm{~F}-\mathrm{NMR}$ analysis. The product was purified by FCC (gradient hexane/ethyl acetate $=99: 1-98: 2$ ) to afford the title compound $\mathbf{6 i}$ as a colourless oil in $90 \%$ yield (64 mg); d.r. of the purified product 20:1; 95\% enantiomeric excess, identical of that of the corresponding starting material (100\% enantiospecificity). HPLC analysis on a Daicel Chiralpak IB column: hexane eluent, flow rate $1.00 \mathrm{~mL} / \mathrm{min}, \lambda=215 \mathrm{~nm}: \tau_{\text {minor }}=5.5$ $\min , \tau_{\text {major }}=6.8 \mathrm{~min}$.

$[\boldsymbol{\alpha}]_{\mathbf{D}} \mathbf{2 4}^{24}+4.9(\mathbf{c}=0.98) \mathbf{R}_{\mathbf{f}}\left(96 / 4 n\right.$-hexane/EtOAc): 0.25; IR (film) $v_{\max } / \mathrm{cm}^{-1}: 2978,1373$, 1315, 1277, 1145, 1093, 700; ${ }^{1} \mathbf{H}$ NMR $\left(\mathrm{CDCl}_{3}, 400 \mathrm{MHz}\right) \delta(\mathrm{ppm}): 7.29$ - 7.21 (m, 2H), 7.21 - $7.14(\mathrm{~m}, 3 \mathrm{H}), 2.88-2.69(\mathrm{~m}, 2 \mathrm{H}), 2.20-1.96(\mathrm{~m}, 4 \mathrm{H}), 1.29$ (d, J = 7.1 Hz, 3H), 1.19 (s, $6 \mathrm{H}), 1.17(\mathrm{~s}, 6 \mathrm{H}) ;{ }^{13} \mathbf{C ~ N M R}\left(\mathrm{CDCl}_{3}, 100 \mathrm{MHz}\right) \delta(\mathrm{ppm}): 144.8,128.1,128.1,127.2(\mathrm{q}, J=$ $277.0 \mathrm{~Hz}), 126.3,83.5,46.7,32.7(\mathrm{q}, J=32.8 \mathrm{~Hz}), 28.2(\mathrm{q}, J=3.3 \mathrm{~Hz}), 28.1(\mathrm{q}, J=3.3 \mathrm{~Hz})$, 24.9, 24.7, 16.6; ${ }^{19} \mathbf{F}$ NMR $\left(\mathrm{CDCl}_{3}, 377 \mathrm{MHz}\right) \delta(\mathrm{ppm}):-73.30$ (d, $\left.J=8.8 \mathrm{~Hz}\right)$. HRMS (EI) mass calculated for $[\mathrm{M}]^{+}\left(\mathrm{C}_{19} \mathrm{H}_{26} \mathrm{O}_{2} \mathrm{BF}_{3}{ }^{+}\right)$requires $\mathrm{m} / \mathrm{z}$ 354.1972, found $\mathrm{m} / \mathrm{z} 354.1967$.

\section{tert-butyl (S)-6-phenyl-4-((1s,3R)-1-(4,4,5,5-tetramethyl-1,3,2-dioxaborolan-2-yl)-3-} (trifluoromethyl)cyclobutyl)hexanoate
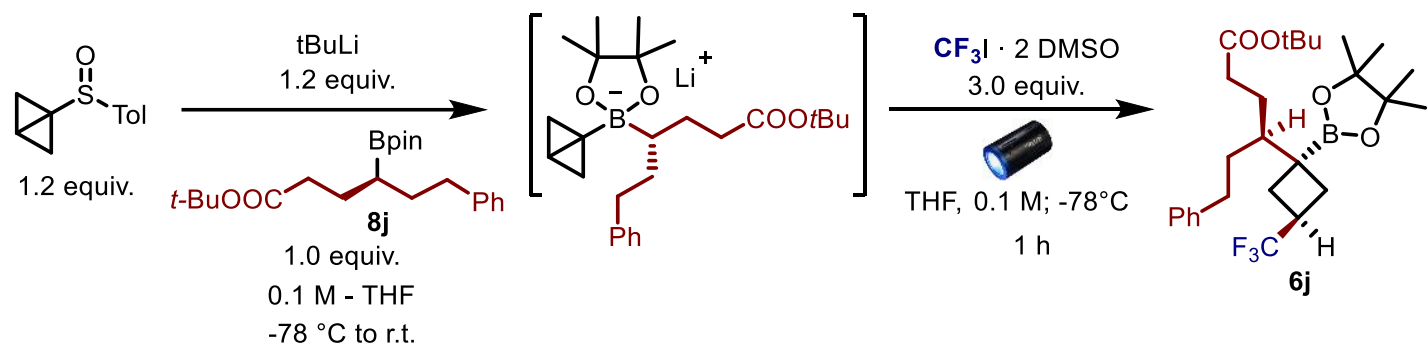

According to the General Procedure, using boronic ester 8j (74.8 mg, 0.20 mmol, 1.0 equiv.) and trifluoromethyl iodide DMSO complex (211 mg, $138 \mu \mathrm{L}, 0.60 \mathrm{mmol}, 3.0$ equiv.). The crude d.r. was measured to be $11: 1$ by ${ }^{19} \mathrm{~F}-\mathrm{NMR}$ analysis. The product was purified by FCC 
(gradient pentane/dichloromethane $=100: 0-90: 10$ ) to afford the title compound $\mathbf{6 j}$ as a colourless oil in $66 \%$ yield $(65 \mathrm{mg})$; d.r. of the purified product $11: 1 ; 92 \%$ enantiomeric excess identical of that of the corresponding starting material (100\% enantiospecificity). HPLC analysis on two consecutive Daicel Chiralpak IA columns: eluent 99.5:0.5 hexane/i-PrOH, flow rate $0.6 \mathrm{~mL} / \mathrm{min}, \lambda=220 \mathrm{~nm}$ : $\tau_{\text {minor }}=15.4 \mathrm{~min}, \tau_{\text {major }}=15.9 \mathrm{~min}$.

$[\boldsymbol{\alpha}]_{\mathbf{D}^{25}}=+5.25(\mathrm{c}=1.0) \mathbf{R}_{\mathbf{f}}\left(95 / 5 n\right.$-hexane/EtOAc): 0.2; IR (film) $v_{\max } / \mathrm{cm}^{-1}: 2978,1729,1379$, 1313, 1277, 1146, 1094, 843, 699; ${ }^{1} \mathbf{H}$ NMR $\left(\mathrm{CDCl}_{3}, 500 \mathrm{MHz}\right) \delta(\mathrm{ppm}): 7.31-7.22$ (m, 2H), $7.22-7.12(\mathrm{~m}, 3 \mathrm{H}), 2.87-2.72(\mathrm{~m}, 1 \mathrm{H}), 2.69-2.49(\mathrm{~m}, 2 \mathrm{H}), 2.36-2.14(\mathrm{~m}, 4 \mathrm{H}), 1.98-1.78$ $(\mathrm{m}, 2 \mathrm{H}), 1.74-1.49(\mathrm{~m}, 4 \mathrm{H}), 1.48-1.40(\mathrm{~m}, 10 \mathrm{H}), 1.26(\mathrm{~s}, 12 \mathrm{H}) ;{ }^{13} \mathbf{C ~ N M R}\left(\mathrm{CDCl}_{3}, 100 \mathrm{MHz}\right)$ $\delta$ (ppm): 173.3, 143.1, 128.4, 128.4, 128.0, $127.0(\mathrm{q}, J=277.0 \mathrm{~Hz}), 125.8,83.5,80.2$, 48.6, 35.0, 34.5, 34.4, 33.3 (q, $J=32.8 \mathrm{~Hz}), 31.1$ (q, $J=3.2 \mathrm{~Hz}), 31.1$ (q, $J=3.2 \mathrm{~Hz}), 28.3,27.5$, 24.9, 24.9; ${ }^{19}$ F NMR $\left(\mathrm{CDCl}_{3}, 377 \mathrm{MHz}\right) \delta(\mathrm{ppm}):-73.35(\mathrm{~d}, J=8.8 \mathrm{~Hz})$. HRMS (ESI) mass calculated for $[\mathrm{M}+\mathrm{Na}]^{+}\left(\mathrm{C}_{27} \mathrm{H}_{40} \mathrm{BF}_{3} \mathrm{NaO}_{4}{ }^{+}\right)$requires $m / z, 519.2869$, found $m / z$ 519.2872.

Tert-butyl (S)-2-((1s,3R)-1-(4,4,5,5-tetramethyl-1,3,2-dioxaborolan-2-yl)-3-(trifluoromet hyl)cyclobutyl)pyrrolidine-1-carboxylate (6k)

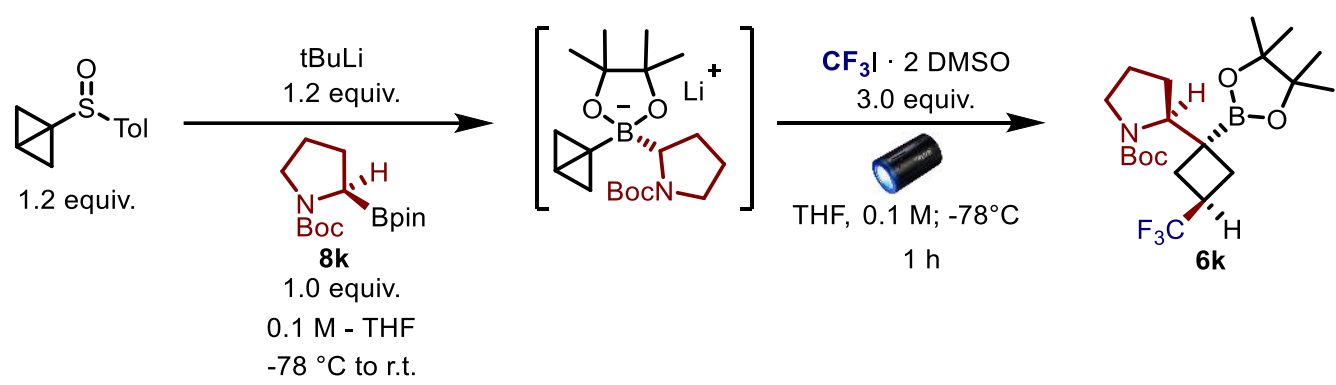

According to the General Procedure, using boronic ester 8k (59.4 mg, $0.20 \mathrm{mmol}, 1.0$ equiv.) and trifluoromethyl iodide DMSO complex (211 mg, $138 \mu \mathrm{L}, 0.60 \mathrm{mmol}, 3.0$ equiv.). The product was purified by FCC (gradient hexane/ethyl acetate $=100: 0-90: 10$ ) to afford the title compound $6 \mathbf{k}$ as a colourless oil in $51 \%$ yield $(43 \mathrm{mg})$.

The enantiomeric excess and the d.r. of compound $\mathbf{6 k}$ was determined by HPLC as detailed following the procedure described in the following section: $90 \%$ enantiomeric excess was measured, identical of that of the corresponding starting material (100\% enantiospecificity) and $>20: 1$ d.r. were observed.

$[\boldsymbol{\alpha}]_{\mathbf{D}}{ }^{25}=-20.2(\mathrm{c}=1.5) \mathbf{R}_{\mathbf{f}}\left(85 / 15 n\right.$-hexane/EtOAc): 0.35; IR (film) $v_{\max } / \mathrm{cm}^{-1}: 2975,1694$, 1381, 1277, 1148; ${ }^{1} \mathbf{H}$ NMR $\left(\mathrm{CDCl}_{3}, 500 \mathrm{MHz}\right)$ broad signals observed due to rotameric equilibria, $\delta(\mathrm{ppm}): 3.97-3.76(\mathrm{~m}, 1 \mathrm{H}), 3.71-3.42(\mathrm{~m}, 1 \mathrm{H}), 3.32-3.13(\mathrm{~m}, 1 \mathrm{H}), 2.95-2.57$ (m, 1H), $2.47-1.87$ (m, 5H), $1.87-1.60$ (m, 3H), 1.45 (bs, 9H), 1.25 (bs, 12H); ${ }^{13} \mathbf{C ~ N M R}$ 
$\left(\mathrm{CDCl}_{3}, 125 \mathrm{MHz}\right)$ spectrum greatly complicated due to rotameric equilibria $\delta(\mathrm{ppm})$ : 155.6, 128.5, 126.3, 83.7, 82.7, 80.0, 63.5, 62.0, 48.1, 32.8 (q, $J=31.7 \mathrm{~Hz}), 31.7,30.6,30.4,29.8$, 28.7, 28.6, 27.4, 25.2, 25.1, 24.9, 24.2, 23.9, 22.8, 22.8.; ${ }^{19} \mathbf{F} \mathbf{N M R}\left(\mathrm{CDCl}_{3}, 377 \mathrm{MHz}\right) \delta(\mathrm{ppm})$ : broad signals observed due to rotameric equilibria $-73.24(\mathrm{~d}, J=8.7 \mathrm{~Hz}$ ). HRMS (ESI) mass calculated for $[\mathrm{M}+\mathrm{H}]^{+}\left(\mathrm{C}_{20} \mathrm{H}_{34} \mathrm{BF}_{3} \mathrm{NO}_{4}{ }^{+}\right)$requires $\mathrm{m} / z$ 420.2531, found $\mathrm{m} / z$ 420.2546.

\section{Determination of enantiomeric excess of compound $\mathbf{6} \boldsymbol{k}-\mathbf{1}$ :}

In order to facilitate the measurement of the enantiomeric excess of compound $\mathbf{6 k}$, a chromophore was introduced by chemical derivatization.

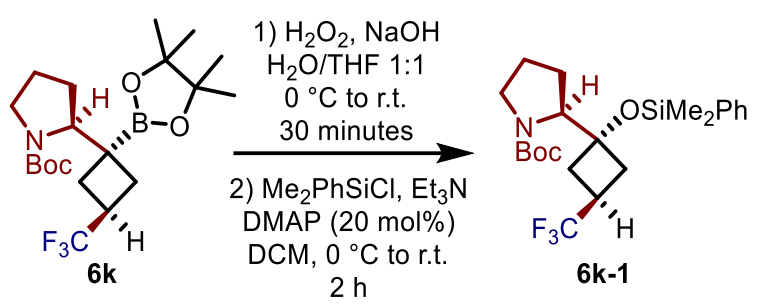

Synthesis of 6k-1: To a stirred solution of boronic ester $6 \mathbf{k}(9 \mathrm{mg}, 0.02 \mathrm{mmol})$ in $500 \mu \mathrm{L}$ of THF at $0{ }^{\circ} \mathrm{C}$ was added $500 \mu \mathrm{L}$ of a $2: 1$ solution of $\mathrm{H}_{2} \mathrm{O}_{2}\left(30 \%\right.$ w/w in $\left.\mathrm{H}_{2} \mathrm{O}\right)$ and $\mathrm{NaOH}(2 \mathrm{M}$ in $\mathrm{H}_{2} \mathrm{O}$ ). The reaction was stirred for 5 minutes at $0{ }^{\circ} \mathrm{C}$, and slowly warmed to room temperature under stirring for 15 minutes, after which it was poured into saturated $\mathrm{NH}_{4} \mathrm{Cl}$ solution $(20 \mathrm{~mL})$ and extracted twice with ethyl acetate $(20 \mathrm{~mL})$. The organic phase was collected, washed with brine $(20 \mathrm{~mL})$ and dried over magnesium sulfate. Solvents were removed under vacuo to afford the crude alcohol intermediate which was used in the next step without further purification.

The residue was placed in a young tube and dissolved in dichloromethane (200 $\mu \mathrm{L})$. Triethyl amine $(7.2 \mu \mathrm{L}, 0.05 \mathrm{mmol})$ and 4-dimethylaminopyridine (0.5 mg, $0.004 \mathrm{mmol}, 20 \mathrm{~mol} \%)$ were added. The solution was cooled to $0{ }^{\circ} \mathrm{C}$ and $\mathrm{Me}_{2} \mathrm{PhSiCl}(8.5 \mu \mathrm{L}, 0.05 \mathrm{mmol}, 2.5$ equiv.) was slowly added under stirring, after which the reaction was warmed to room temperature for 1 hour. The reaction was filtered over a thin layer of silica and eluted with diethyl ether. The solvents were removed under vacuo to afford the crude residue, which was purified by FCC (gradient hexane/ethyl acetate $=99: 1-98: 2)$ to afford silyl ether 6k-1 (8 mg, $0.018 \mathrm{mmol}, 90 \%$ yield for two steps) as a colourless oil, 90\% enantiomeric excess. HPLC analysis on a Daicel Chiralpak IC column: eluent 99.85:0.15 hexane/i-PrOH, flow rate $1.0 \mathrm{~mL} / \mathrm{min}, \lambda=215 \mathrm{~nm}$ : $\tau_{\text {minor }}=27.7 \mathrm{~min}, \tau_{\text {major }}=30.8 \mathrm{~min}$.

$[\boldsymbol{\alpha}] \mathbf{D}^{25}=-8.9(\mathbf{c}=0.4) \mathbf{R}_{\mathbf{f}}\left(85 / 15 n\right.$-hexane/EtOAc): 0.9; IR (film) $v_{\max } / \mathrm{cm}^{-1}: 2653,2929,1694$, 1387, 1270, 1152, 1008, 829; ${ }^{1} \mathbf{H}$ NMR ( $\left.\mathrm{CDCl}_{3}, 500 \mathrm{MHz}\right) \delta$ (ppm): broad signals observed due to rotameric equilibria, $7.67-7.54(\mathrm{~m}, 2 \mathrm{H}), 7.44-7.31(\mathrm{~m}, 3 \mathrm{H}), 4.02-3.86(\mathrm{~m}, 1 \mathrm{H}), 3.70$ 
- $3.39(\mathrm{~m}, 1 \mathrm{H}), 3.34-3.23(\mathrm{~m}, 1 \mathrm{H}), 2.77-2.47(\mathrm{~m}, 2 \mathrm{H}), 2.45-2.22(\mathrm{~m}, 1 \mathrm{H}), 2.19-2.03(\mathrm{~m}$, 2H), $1.96-1.64(\mathrm{~m}, 4 \mathrm{H}), 1.46(\mathrm{~s}, 9 \mathrm{H}), 0.49-0.37(\mathrm{~m}, 6 \mathrm{H}) ;{ }^{13} \mathbf{C} \mathbf{~ N M R}\left(\mathrm{CDCl}_{3}, 125 \mathrm{MHz}\right)$ $\delta(\mathrm{ppm})$ : complex spectrum due to rotameric equilibria 155.9, 155.2, 139.2, 138.9, 133.2, 131.0, 129.9, 129.8, 128.8, 128.2, 128.1, 126.6, 124.4, 80.1, 79.4, 78.9, 78.7, 63.9, 48.3, 47.9, 33.5, 33.3, 32.1, 31.7, 30.7, 30.4, 30.2, 30.1, 29.9, 29.6, 29.5, 28.6, 26.9, 26.3, 24.3, 23.5, 22.8, $14.3,0.7,0.6,0.5 ;{ }^{19} \mathbf{F ~ N M R}\left(\mathrm{CDCl}_{3}, 470 \mathrm{MHz}\right) \delta(\mathrm{ppm})$ : rotameric equilibria observed -73.2 - -73.6 (m). HRMS (ESI) mass calculated for $[\mathrm{M}+\mathrm{H}]^{+}\left(\mathrm{C}_{22} \mathrm{H}_{33} \mathrm{~F}_{3} \mathrm{NO}_{3} \mathrm{Si}^{+}\right)$requires $\mathrm{m} / \mathrm{z}$ 444.2182, found $\mathrm{m} / \mathrm{z} 444.2212$.

\section{2-((1s,3S)-1-((1R,2S,5R)-2-isopropyl-5-methylcyclohexyl)-3-(trifluoromethyl)cyclobutyl)}

\section{-4,4,5,5-tetramethyl-1,3,2-dioxaborolane (61)}

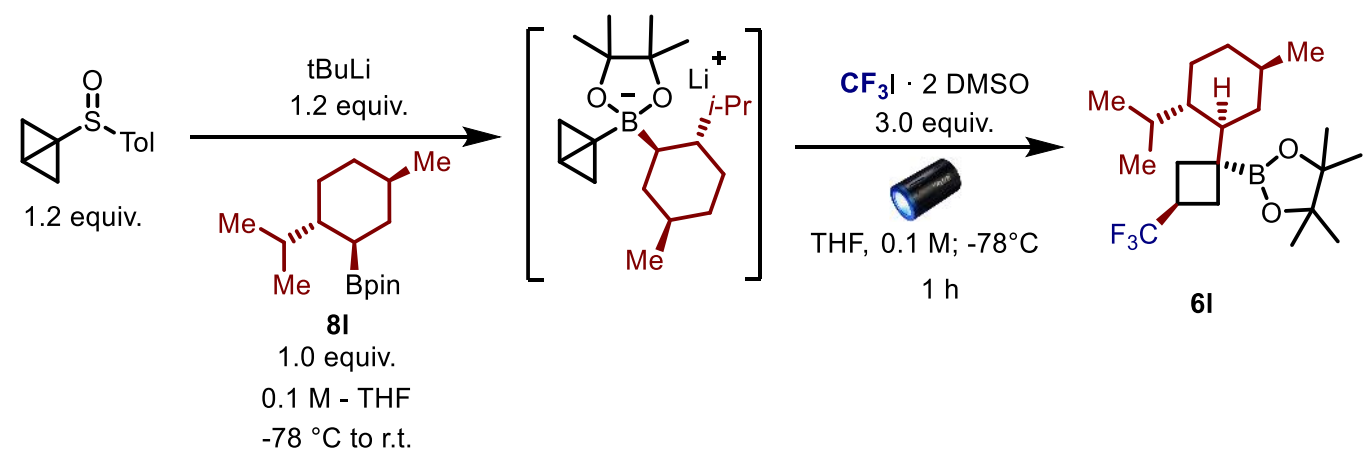

According to the General Procedure, using boronic ester 81 (53 mg, $0.20 \mathrm{mmol}, 1.0$ equiv.) and trifluoromethyl iodide DMSO complex (211 mg, $138 \mu \mathrm{L}, 0.60 \mathrm{mmol}, 3.0$ equiv.). The crude d.r. was measured to be $>20: 1$ by ${ }^{19} \mathrm{~F}-\mathrm{NMR}$ analysis. The product was purified by FCC (gradient hexane/dichloromethane $=90: 10-85: 15$ ) to afford the title compound $\mathbf{6} \mathbf{l}$ as a white solid in $61 \%$ yield $(47 \mathrm{mg})$; d.r. of the purified product $>20: 1$.

$[\boldsymbol{\alpha}]_{\mathbf{D}}{ }^{24}=-12.6(\mathrm{c}=1.1)$ MP: $75-76{ }^{\circ} \mathrm{C}(n$-hexane $) ; \mathbf{R}_{\mathbf{f}}(70 / 30 n$-hexane/dichloromethane): 0.4; IR (film) $v_{\max } / \mathrm{cm}^{-1}: 2951,2929,1371,1309,1274,1141,1094 ;{ }^{1} \mathbf{H} \mathbf{~ N M R}\left(\mathrm{CDCl}_{3}, 500 \mathrm{MHz}\right)$ $\delta(\mathrm{ppm}): 2.76-2.60(\mathrm{~m}, 1 \mathrm{H}), 2.43-2.32(\mathrm{~m}, 1 \mathrm{H}), 2.20-2.10(\mathrm{~m}, 1 \mathrm{H}), 1.97(\mathrm{t}, J=10.4 \mathrm{~Hz}$ $1 \mathrm{H}), 1.88(\mathrm{t}, J=10.6 \mathrm{~Hz}, 1 \mathrm{H}), 1.78$ (heptd, $J=6.9,1.8 \mathrm{~Hz}, 1 \mathrm{H}), 1.72-1.63(\mathrm{~m}, 1 \mathrm{H}), 1.63-$ $1.52(\mathrm{~m}, 2 \mathrm{H}), 1.36-1.20(\mathrm{~m}, 15 \mathrm{H}), 1.11-1.03(\mathrm{~m}, 1 \mathrm{H}), 0.95-0.83(\mathrm{~m}, 7 \mathrm{H}), 0.83-0.68(\mathrm{~m}$, 5H). ${ }^{13} \mathbf{C ~ N M R}\left(\mathrm{CDCl}_{3}, 125 \mathrm{MHz}\right) \delta(\mathrm{ppm}): 127.0(\mathrm{q}, J=275.7 \mathrm{~Hz}), 83.3,50.9,48.5,37.6$, $35.7,33.8(\mathrm{q}, J=30.5 \mathrm{~Hz}), 33.8(\mathrm{q}, J=3.1 \mathrm{~Hz}), 33.4,30.2(\mathrm{q}, J=3.1 \mathrm{~Hz}), 27.6,25.1,24.9$, 24.8, 22.9, 22.0, 16.3; ${ }^{19} \mathbf{F ~ N M R}\left(\mathrm{CDCl}_{3}, 376 \mathrm{MHz}\right) \delta$ (ppm): -73.17 (d, $\left.J=8.9 \mathrm{~Hz}\right) . \mathbf{H R M S}$ (APCI) mass calculated for $[\mathrm{M}+\mathrm{H}]^{+}\left(\mathrm{C}_{21} \mathrm{H}_{36} \mathrm{~F}_{3} \mathrm{O}_{2} \mathrm{~B}^{+}\right)$requires $\mathrm{m} / \mathrm{z} 389.2833$ found $\mathrm{m} / \mathrm{z}$ 389.2844 . 
4,4,5,5-tetramethyl-2-((1s,3S)-3-(trifluoromethyl)-1-((1S,2R,3S,6R)-3,7,7-trimethylbicycl o[4.1.0]heptan-2-yl)cyclobutyl)-1,3,2-dioxaborolane (6m)

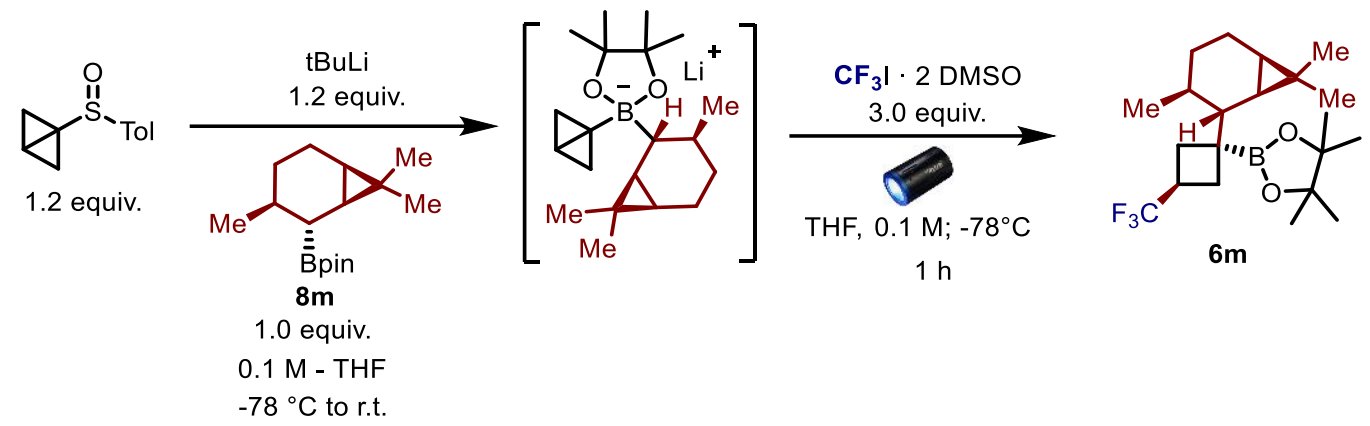

According to the General Procedure, using boronic ester $\mathbf{8 m}$ (52.8 mg, $0.20 \mathrm{mmol}$, 1.0 equiv.) and trifluoromethyl iodide DMSO complex (211 mg, $138 \mu \mathrm{L}, 0.60 \mathrm{mmol}, 3.0$ equiv.). The crude d.r. was measured to be $5: 1$ by ${ }^{19} \mathrm{~F}-\mathrm{NMR}$ analysis. The product was purified by FCC (gradient hexane/dichloromethane $=95: 5-90: 10$ ) to afford the title compound $\mathbf{6 m}$ as a white solid in $71 \%$ yield $(55 \mathrm{mg})$; d.r. of the purified product $>20: 1$.

$[\alpha]_{\mathbf{D}^{25}}=+5.2(\mathrm{c}=0.8)$ MP: $92-94{ }^{\circ} \mathrm{C}\left(n\right.$-hexane); $\mathbf{R}_{\mathbf{f}}(70 / 30 n$-hexane/dichloromethane $): 0.4$; IR (film) $v_{\max } / \mathrm{cm}^{-1}: 2925,2862,1378,1371,1277,1142,1089 ;{ }^{1} \mathbf{H} \mathbf{~ N M R}\left(\mathrm{CDCl}_{3}, 500 \mathrm{MHz}\right)$ $\delta(\mathrm{ppm}): \delta 2.86-2.71(\mathrm{~m}, 1 \mathrm{H}), 2.42-2.32(\mathrm{~m}, 1 \mathrm{H}), 2.30-2.22(\mathrm{~m}, 1 \mathrm{H}), 1.91(\mathrm{t}, J=10.5 \mathrm{~Hz}$, $1 \mathrm{H}), 1.84(\mathrm{t}, J=10.6 \mathrm{~Hz}, 1 \mathrm{H}), 1.80-1.71(\mathrm{~m}, 1 \mathrm{H}), 1.61-1.56(\mathrm{~m}, 1 \mathrm{H}), 1.40-1.32(\mathrm{~m}, 1 \mathrm{H})$, $1.26(\mathrm{~s}, 6 \mathrm{H}), 1.26(\mathrm{~s}, 6 \mathrm{H}), 1.23-1.18(\mathrm{~m}, 1 \mathrm{H}), 0.98(\mathrm{~s}, 3 \mathrm{H}), 0.93(\mathrm{~s}, 3 \mathrm{H}), 0.89-0.81(\mathrm{~m}, 2 \mathrm{H})$, $0.76(\mathrm{~d}, J=6.7 \mathrm{~Hz}, 3 \mathrm{H}), 0.73-0.66(\mathrm{~m}, 1 \mathrm{H}), 0.48-0.42(\mathrm{~m}, 1 \mathrm{H}), 0.35(\mathrm{dd}, J=9.3,5.5 \mathrm{~Hz}$, $1 \mathrm{H}) ;{ }^{13} \mathrm{C} \mathrm{NMR}\left(\mathrm{CDCl}_{3}, 125 \mathrm{MHz}\right) \delta(\mathrm{ppm}): 127.0(\mathrm{q}, J=277.1 \mathrm{~Hz}), 83.3,50.3,33.9,33.7$, $33.5(\mathrm{q}, J=30.9 \mathrm{~Hz}), 29.5$ (q, $J=3.4 \mathrm{~Hz}), 29.1,25.5,25.0,24.7,20.5,19.7,19.4$, 17.5, 16.3; ${ }^{19} \mathbf{F}$ NMR $\left(\mathrm{CDCl}_{3}, 470 \mathrm{MHz}\right) \delta(\mathrm{ppm}):-73.5(\mathrm{~d}, J=9.1 \mathrm{~Hz})$. HRMS (APCI) mass calculated for $[\mathrm{M}+\mathrm{H}]^{+}\left(\mathrm{C}_{21} \mathrm{H}_{36} \mathrm{~F}_{3} \mathrm{O}_{2} \mathrm{~B}^{+}\right)$requires $m / z, 387.2677$ found $m / z$ 387.2662.

tert-butyl(((2S,4S,6S)-2,4-dimethyl-6-((1s,3R)-1-(4,4,5,5-tetramethyl-1,3,2-dioxaborolan2-yl)-3-(trifluoromethyl)cyclobutyl)heptyl)oxy)diphenylsilane (6n)

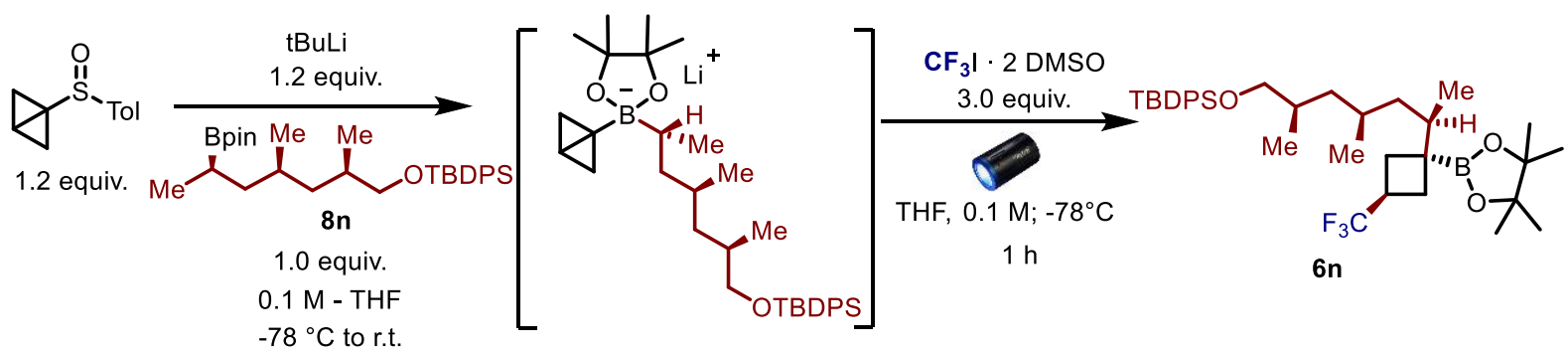

According to the General Procedure, using boronic ester 8n (102 mg, $0.20 \mathrm{mmol}, 1.0$ equiv.) and trifluoromethyl iodide DMSO complex (211 mg, $138 \mu \mathrm{L}, 0.60 \mathrm{mmol}, 3.0$ equiv.). The 
crude d.r. was measured to be $>20: 1$ by ${ }^{19} \mathrm{~F}-\mathrm{NMR}$ analysis. The product was purified by FCC (gradient hexane/dichloromethane $=85: 15-80: 20$ ) to afford the title compound $\mathbf{6 n}$ as a colourless oil in $76 \%$ yield (96 mg); d.r. of the purified product $>20: 1$.

$[\boldsymbol{\alpha}] \mathbf{D}^{25}=+17.9(\mathrm{c}=1.1) \mathbf{R}_{\mathbf{f}}\left(70 / 30 n\right.$-hexane/dichloromethane): 0.4; IR (film) $v_{\max } / \mathrm{cm}^{-1}: 2958$, 2858, 1379, 1143, 1112, 701; ${ }^{1} \mathbf{H}$ NMR $\left(\mathrm{CDCl}_{3}, 400 \mathrm{MHz}\right) \delta(\mathrm{ppm}): \delta 7.77-7.57(\mathrm{~m}, 4 \mathrm{H})$, $7.53-7.30(\mathrm{~m}, 6 \mathrm{H}), 3.50(\mathrm{dd}, J=9.8,5.1 \mathrm{~Hz}, 1 \mathrm{H}), 3.43(\mathrm{dd}, J=9.8,6.4 \mathrm{~Hz}, 1 \mathrm{H}), 2.87-2.66$ (m, 1H), 2.30-2.15 (m, 2H)), $1.97-1.66(\mathrm{~m}, 3 \mathrm{H}), 1.57-1.43(\mathrm{~m}, 2 \mathrm{H}), 1.33-1.28(\mathrm{~m}, 1 \mathrm{H})$, $1.25(\mathrm{~s}, 12 \mathrm{H}), 1.15-1.09(\mathrm{~m}, 1 \mathrm{H}), 1.06(\mathrm{~s}, 9 \mathrm{H}), 1.00-0.96(\mathrm{~m}, 1 \mathrm{H}), 0.94(\mathrm{~d}, J=6.6 \mathrm{~Hz}, 3 \mathrm{H})$, $0.91-0.84(\mathrm{~m}, 1 \mathrm{H}), 0.84-0.78(\mathrm{~m}, 6 \mathrm{H}) ;{ }^{13} \mathbf{C} \mathbf{N M R}\left(\mathrm{CDCl}_{3}, 100 \mathrm{MHz}\right) \delta(\mathrm{ppm}): 135.7,135.7$, 134.2, 134.2, 129.6 (x2), 127.7 (x2), 127.0 (q, $J=277.0$ Hz), 83.4, 69.1, 42.4, 40.7, 39.6, 33.2, $32.6(\mathrm{q}, J=31.0 \mathrm{~Hz}), 29.9,29.6(\mathrm{q}, J=3.0 \mathrm{~Hz}), 29.1(\mathrm{q}, J=3.0 \mathrm{~Hz}), 27.3,27.0,24.9,19.8$, 19.5, 17.5, 14.6.; ${ }^{19} \mathbf{F}$ NMR $\left(\mathrm{CDCl}_{3}, 376 \mathrm{MHz}\right) \delta(\mathrm{ppm}):-73.3(\mathrm{~d}, J=9.0 \mathrm{~Hz})$. HRMS (APCI) mass calculated for $[\mathrm{M}+\mathrm{H}]^{+}\left(\mathrm{C}_{36} \mathrm{H}_{54} \mathrm{~F}_{3} \mathrm{O}_{3} \mathrm{BSi}^{+}\right)$requires $m / z$ 631.3960, found $m / z 631.3983$.

Tert-butyldimethyl(((3S,5R,8S,10S,13R,14R,17R)-8,10,13-trimethyl-17-((R)-6-methylhe ptan-2-yl)-6-((1s,3S)-1-(4,4,5,5-tetramethyl-1,3,2-dioxaborolan-2-yl)-3-(trifluoromethyl) cyclobutyl)hexadecahydro-1H-cyclopenta[a]phenanthrene-3-yl)oxy)silane (6o)

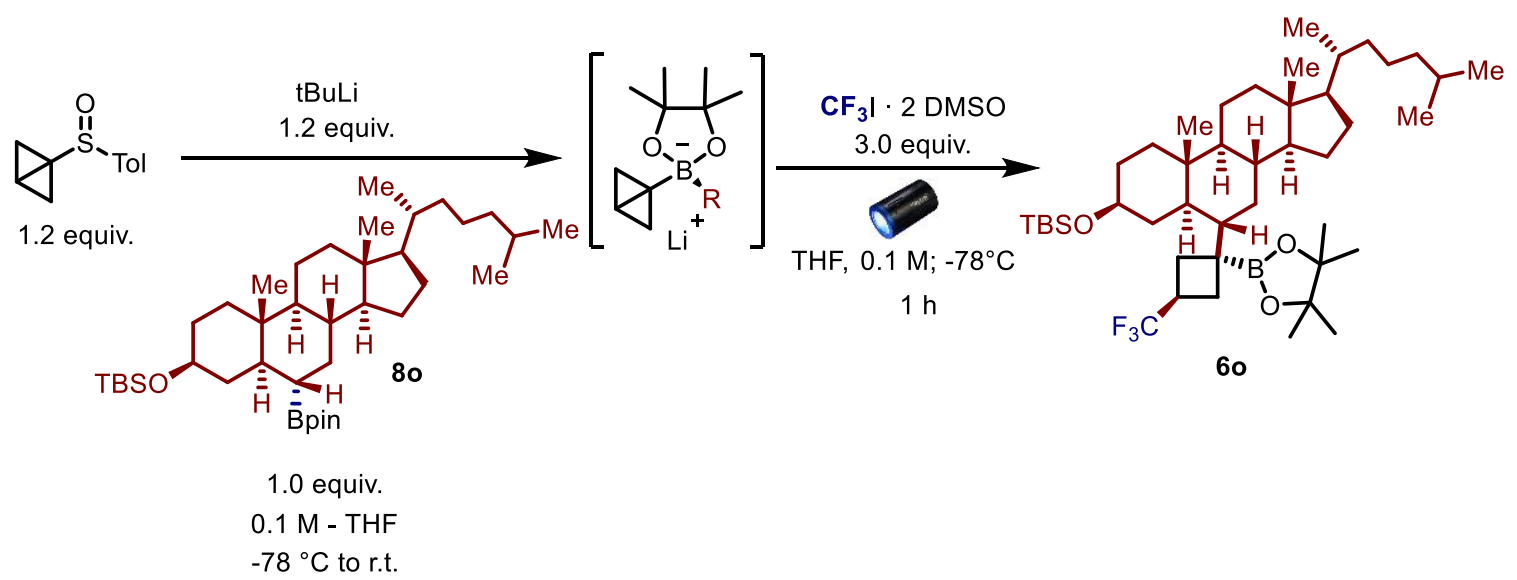

According to the General Procedure, using boronic ester 80 (125.8 mg, $0.20 \mathrm{mmol}, 1.0$ equiv.) and trifluoromethyl iodide DMSO complex (211 mg, $138 \mu \mathrm{L}, 0.60 \mathrm{mmol}, 3.0$ equiv.). The crude d.r. was measured to be $>20: 1$ by ${ }^{19} \mathrm{~F}-\mathrm{NMR}$ analysis. The product was purified by FCC (gradient hexane/dichloromethane $=95: 5-85: 15$ ) to afford the title compound $\mathbf{6 0}$ as a white solid in $67 \%$ yield $(100 \mathrm{mg})$; d.r. of the purified product $>20: 1$.

$[\alpha]_{\mathbf{D}}{ }^{25}=+20.5(\mathrm{c}=1.1)$ MP: $81-83{ }^{\circ} \mathrm{C}$ ( $n$-hexane); $\mathbf{R}_{\mathbf{f}}(70 / 30 n$-hexane/dichloromethane): 0.4; IR (film) $v_{\max } / \mathrm{cm}^{-1}: 2930,2855,1378,1151,1091,835,758,670 ;{ }^{1} \mathbf{H} \mathbf{~ N M R}\left(\mathrm{CDCl}_{3}, 500 \mathrm{MHz}\right)$ $\delta$ (ppm): 3.42 (tt, $J=10.4,4.7 \mathrm{~Hz}, 1 \mathrm{H}), 2.83-2.65(\mathrm{~m}, 1 \mathrm{H}), 2.40-2.09$ (m, 2H), $2.00-1.75$ (m, 4H), $1.68-1.42(\mathrm{~m}, 8 \mathrm{H}), 1.42-1.19(\mathrm{~m}, 21 \mathrm{H}), 1.19-0.92(\mathrm{~m}, 11 \mathrm{H}), 0.92-0.82(\mathrm{~m}$, 
19H), $0.81(\mathrm{~s}, 3 \mathrm{H}), 0.66-0.46(\mathrm{~m}, 5 \mathrm{H}), 0.07-0.03(\mathrm{~m}, 6 \mathrm{H}) .{ }^{13} \mathbf{C ~ N M R}\left(\mathrm{CDCl}_{3}, 125 \mathrm{MHz}\right)$ $\delta$ (ppm): $\delta 127.0(\mathrm{q}, J=276.1 \mathrm{~Hz}), 83.3,72.4,56.5,56.4,54.8,49.5,48.7,42.8,40.2,39.7$, 37.6, 36.4, 35.9, 35.9, 35.7, 35.5, 34.0, 33.8 (q, $J=31.5 \mathrm{~Hz}), 31.6,28.4$, 28.2, 26.2, 25.0, 24.8, 24.4, 24.0, 23.0, 22.7, 21.5, 18.9, 18.4, 13.6, 12.2, -4.2, -4.4.; ${ }^{\mathbf{1}} \mathbf{F} \mathbf{N M R}\left(\mathrm{CDCl}_{3}, 376 \mathrm{MHz}\right)$ $\delta(\mathrm{ppm}):-73.18(\mathrm{~d}, J=8.8 \mathrm{~Hz})$. HRMS (MALDI) mass calculated for $[\mathrm{M}+\mathrm{Na}]^{+}$ $\left(\mathrm{C}_{44} \mathrm{H}_{78} \mathrm{~F}_{3} \mathrm{O}_{3} \mathrm{SiBNa}^{+}\right)$requires $m / z 773.5694$ found $m / z, 773.5701$.

2-((1s,3s)-1-cyclohexyl-3-((1,1,1,2,3,3,4,4,5,5,6,6,7,7,8,8-hexadecafluoro-8 $\lambda^{3}$-octan-2-yl)$\lambda^{2}$-fluoraneyl)cyclobutyl)-4,4,5,5-tetramethyl-1,3,2-dioxaborolane (6p)

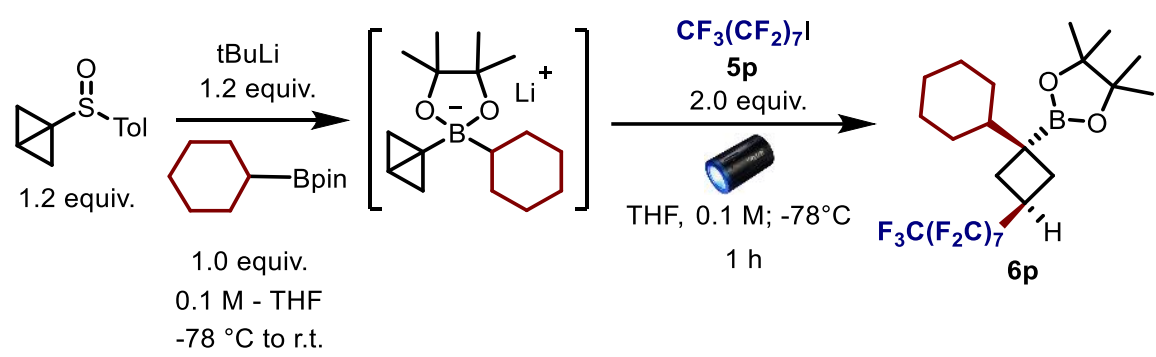

According to the General Procedure, using cyclohexyl boronic acid pinacol ester $(45 \mu \mathrm{L}$, $0.20 \mathrm{mmol}, 1.0$ equiv.) and perfluorooctyl iodide $5 \mathbf{p}(218 \mathrm{mg}, 105 \mu \mathrm{L}, 0.40 \mathrm{mmol}, 2.0$ equiv.). The photochemical reaction was found to be significantly more efficient when performed in the presence of $1 \mathrm{~mol} \%$ of $\mathrm{Ru}(\mathrm{bpy})_{3}\left(\mathrm{PF}_{6}\right)_{2}(1.7 \mathrm{mg}, 0.002 \mathrm{mmol})$, which was added as a solid to the cold mixture prior to the addition of perfluorooctyl iodide. The crude d.r. was measured to be $>20: 1$ by ${ }^{19} \mathrm{~F}-\mathrm{NMR}$ analysis. The product was purified by FCC (gradient hexane/dichloromethane $=99: 1-98: 2$ ) to afford the title compound $\mathbf{6 p}$ as a white solid in 74\% yield (51 mg); d.r. of the purified product $>20: 1$.

MP: $71-72{ }^{\circ} \mathrm{C}$ ( $n$-hexane); $\mathbf{R}_{\mathbf{f}}$ (90:10 $n$-hexane/dichloromethane): 0.8 ; IR (film) $v_{\max } / \mathrm{cm}^{-1}$ : 2923, 2852, 1202, 1143, 754, 656; ${ }^{1} \mathbf{H}$ NMR $\left(\mathrm{CDCl}_{3}, 500 \mathrm{MHz}\right) \delta(\mathrm{ppm}): 2.97$ - $2.72(\mathrm{~m}, 1 \mathrm{H})$, $2.26-2.11(\mathrm{~m}, 2 \mathrm{H}), 1.99-1.83(\mathrm{~m}, 2 \mathrm{H}), 1.78-1.54(\mathrm{~m}, 5 \mathrm{H}), 1.32-1.23(\mathrm{~m}, 13 \mathrm{H}), 1.23-$ $1.02(\mathrm{~m}, 3 \mathrm{H}), 0.96(\mathrm{qd}, J=12.5,3.2 \mathrm{~Hz}, 2 \mathrm{H}) \cdot{ }^{13} \mathbf{C} \mathbf{N M R}\left(\mathrm{CDCl}_{3}, 125 \mathrm{MHz}\right) \delta(\mathrm{ppm}): 83.5,48.4$, $31.41(\mathrm{t}, J=25.0 \mathrm{~Hz}), 28.8(\mathrm{t}, J=4.5 \mathrm{~Hz}), 28.7,26.8(\mathrm{x} 2), 24.9 ;{ }^{19} \mathbf{F} \mathbf{N M R}\left(\mathrm{CDCl}_{3}, 470 \mathrm{MHz}\right)$ $\delta$ (ppm): -80.56 - -81.04 (m, 3F), -120.00 (d, J = 14.9 Hz, 1F), -120.1 (d, J = 14.9 Hz, 1F), $121.54--122.34(\mathrm{~m}, 6 \mathrm{~F}),-122.68--122.84(\mathrm{~m}, 2 \mathrm{~F}),-122.90--123.12(\mathrm{~m}, 2 \mathrm{~F}),-125.91--$ $126.58(\mathrm{~m}, 2 \mathrm{~F})$. HRMS (EI) mass calculated for $\left[\mathrm{M}-\mathrm{CH}_{3}\right]^{+}\left(\mathrm{C}_{24} \mathrm{H}_{28} \mathrm{~F}_{17} \mathrm{O}_{2} \mathrm{~B}^{+}\right)$requires $\mathrm{m} / \mathrm{z}$ 667.1671 found $\mathrm{m} / \mathrm{z} 667.1700$. 
2-((1s,3s)-3-cyclohexyl-3-(4,4,5,5-tetramethyl-1,3,2-dioxaborolan-2-yl)cyclobutyl)acetoni trile (6q)

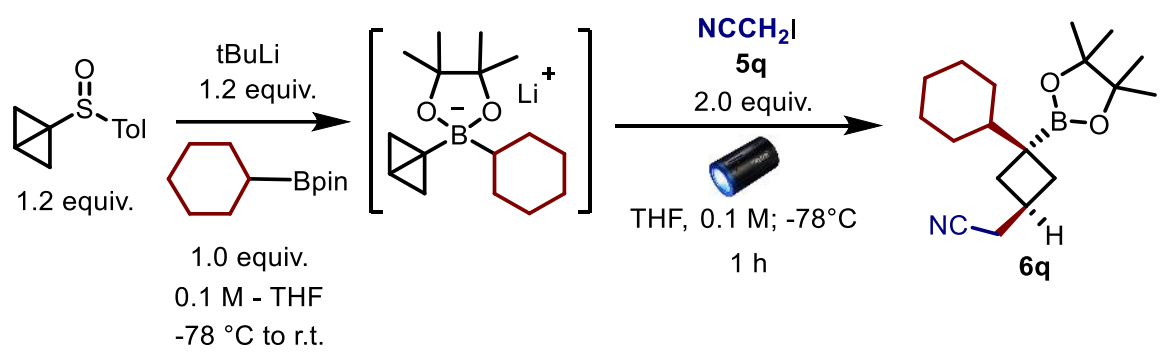

According to the General Procedure, using cyclohexyl boronic acid pinacol ester $(45 \mu \mathrm{L}$, $0.20 \mathrm{mmol}, 1.0$ equiv.) and iodoacetonitrile $\mathbf{5 q}$ ( $29 \mu \mathrm{L}, 0.40 \mathrm{mmol}, 2.0$ equiv.). The crude d.r. was measured to be $3: 1$ by ${ }^{1} \mathrm{H}-\mathrm{NMR}$ analysis and confirmed by GC-MS. The product was purified by FCC (gradient hexane/ethyl acetate $=100: 0-95: 5$ ). Traces of iodoacetonitrile were distilled out $\left(50{ }^{\circ} \mathrm{C} ; 1 \mathrm{mBar}\right)$ to afford the title compound $\mathbf{6 q}$ as a in $75 \%$ yield $(46 \mathrm{mg})$ as a white solid; d.r. of the purified product 3:1.

MP: $52-54{ }^{\circ} \mathrm{C}$ ( $n$-hexane/EtOAc); $\mathbf{R}_{\mathbf{f}}$ (85:15 $n$-hexane/ethyl acetate): 0.4; IR (film) $v_{\max } / \mathrm{cm}^{-1}$ : 2922, 2850, 2246, 1386, 1309, 1142, 965, 859; ${ }^{1} \mathbf{H}$ NMR $\left(\mathrm{CDCl}_{3}, 500 \mathrm{MHz}\right) \delta(\mathrm{ppm})$ (major distereoisomer): $2.43-2.36(\mathrm{~m}, 1 \mathrm{H}), 2.34(\mathrm{~d}, J=6.2 \mathrm{~Hz}, 2 \mathrm{H}), 2.33-2.26(\mathrm{~m}, 2 \mathrm{H}), 1.77-1.50$ $(\mathrm{m}, 7 \mathrm{H}), 1.30-1.21(\mathrm{~m}, 13 \mathrm{H}), 1.23-1.06(\mathrm{~m}, 3 \mathrm{H}), 0.93(\mathrm{qd}, J=12.6,3.5 \mathrm{~Hz}, 2 \mathrm{H}) ;{ }^{13} \mathbf{C ~ N M R}$ $\left(\mathrm{CDCl}_{3}, 125 \mathrm{MHz}\right) \delta(\mathrm{ppm})$ (major distereoisomer): 118.9, 83.3, 48.9, 35.4, 29.0, 27.5, 26.8, 24.9 (x2), 24.1; HRMS (ESI) mass calculated for $[\mathrm{M}+\mathrm{H}]^{+}\left(\mathrm{C}_{18} \mathrm{H}_{31} \mathrm{NBO}_{2}{ }^{+}\right)$requires $\mathrm{m} / \mathrm{z}$ 304.2446, found $\mathrm{m} / \mathrm{z} 304.2448$.

Ethyl 2-((1s,3s)-3-cyclohexyl-3-(4,4,5,5-tetramethyl-1,3,2-dioxaborolan-2-yl)cyclobutyl)a cetate $(6 \mathbf{r})$
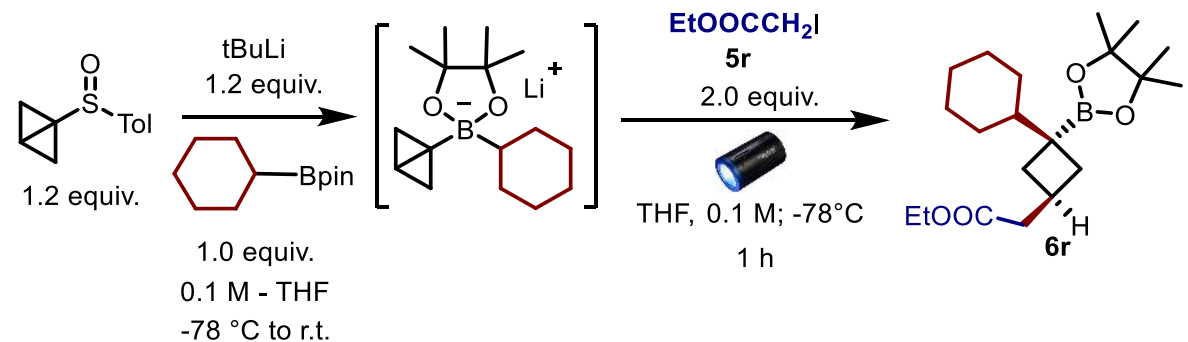

According to the General Procedure, using cyclohexyl boronic acid pinacol ester (45 $\mu \mathrm{L}$, $0.20 \mathrm{mmol}, 1.0$ equiv.) and ethyl iodoacetate ( $47 \mu \mathrm{L}, 0.40 \mathrm{mmol}, 2.0$ equiv.). The crude d.r. was measured to be 5.3:1 by ${ }^{1} \mathrm{H}-\mathrm{NMR}$ analysis and confirmed by GC-MS. The product was purified by FCC (gradient hexane/ethyl acetate $=100: 0-97.5: 2.5$ ) to afford the title compound 6r as a colourless oil in 64\% yield (45 mg); d.r. of the purified product 5.3:1. 
$\mathbf{R}_{\mathbf{f}}$ (90:10 $n$-hexane/ethyl acetate): 0.3; IR (film) $v_{\max } / \mathrm{cm}^{-1}: 2922,2850,1734,1384,1307$, 1142, 859; ${ }^{1} \mathbf{H}$ NMR $\left(\mathrm{CDCl}_{3}, 500 \mathrm{MHz}\right) \delta$ (ppm) (major diastereoisomer): 4.09 (q, $J=7.1 \mathrm{~Hz}$, $2 \mathrm{H}), 2.47-2.36(\mathrm{~m}, 1 \mathrm{H}), 2.33-2.25(\mathrm{~m}, 4 \mathrm{H}), 1.74-1.58(\mathrm{~m}, 5 \mathrm{H}), 1.50-1.33(\mathrm{~m}, 2 \mathrm{H}), 1.27$ - 1.20 (m, 16H), 1.20 - 0.99 (m, 3H), $0.97-0.86(\mathrm{~m}, 2 \mathrm{H}) ;{ }^{13} \mathbf{C} \mathbf{~ N M R}\left(\mathrm{CDCl}_{3}, 125 \mathrm{MHz}\right)$ $\delta(\mathrm{ppm})$ (major diastereoisomer): 173.4, 83.6, 60.6, 49.8, 42.7, 36.7, 29.6, 28.5, 27.4, 27.3, 25.3, 14.9; HRMS (ESI) mass calculated for $[\mathrm{M}+\mathrm{H}]^{+}\left(\mathrm{C}_{20} \mathrm{H}_{36} \mathrm{O}_{4}{ }^{+}\right)$requires $\mathrm{m} / \mathrm{z}$ 351.2705, found $m / z 351.2696$.

2-((1s,3s)-3-cyclohexyl-3-(4,4,5,5-tetramethyl-1,3,2-dioxaborolan-2-yl)cyclobutyl)acetam ide (6s)

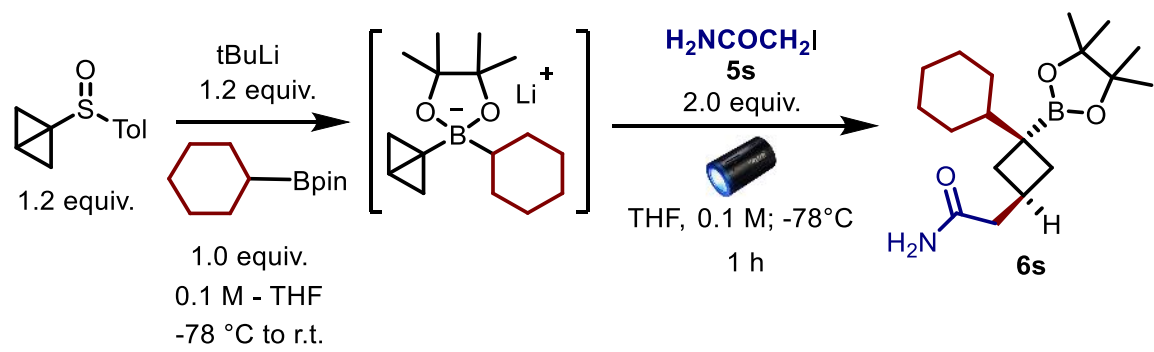

According to the General Procedure, using cyclohexyl boronic acid pinacol ester (45 $\mu \mathrm{L}$, $0.20 \mathrm{mmol}, 1.0$ equiv.) and iodoacetamide (74 mg, $5 \mathrm{~s} \mu \mathrm{L}, 0.40 \mathrm{mmol}, 2.0$ equiv.). After the reaction, the silica plug was substituted with the following work-up. The reaction mixture was diluted with ethyl acetate $(100 \mathrm{~mL})$, washed with water $(50 \mathrm{~mL} \times 2), 10 \%$ sodium thiosulfate solution $(50 \mathrm{~mL})$ and brine $(50 \mathrm{~mL})$. The organic phase was dried over magnesium sulfate and the volatiles were removed under vacuo. The crude d.r. of the product was measured by ${ }^{1} \mathrm{H}$ NMR and found to be 6.5:1. The crude product was purified by FCC (hexane/ethyl acetate $=65: 35-50: 50)$ to afford the title compound $\mathbf{6 s}$ as a white solid in 51\% yield (33 mg); d.r. of the purified product $6.5: 1$.

MP: $175-177{ }^{\circ} \mathrm{C}$ ( $n$-hexane/EtOAc); Rf (70:30 $n$-hexane/ethyl acetate): 0.15; IR (neat) $v_{\max } / \mathrm{cm}^{-1}: 3350,3183,2921,1660,1384,1291,1141,857,674 ;{ }^{1} \mathbf{H} \mathbf{~ N M R}\left(\mathrm{CDCl}_{3}, 400 \mathrm{MHz}\right)$ $\delta(\mathrm{ppm})$ (major diastereoisomer): 5.75 (bs, 1H), 5.42 (bs, 1H), $2.44-2.25(\mathrm{~m}, 3 \mathrm{H}), 2.22$ (d, $J$ $=6.8 \mathrm{~Hz}, 2 \mathrm{H}), 1.74-1.53(\mathrm{~m}, 5 \mathrm{H}), 1.47-1.35(\mathrm{~m}, 2 \mathrm{H}), 1.23(\mathrm{~s}, 12 \mathrm{H}), 1.18-1.01(\mathrm{~m}, 4 \mathrm{H})$, $0.80-0.95(\mathrm{~m}, 2 \mathrm{H}) ;{ }^{13} \mathbf{C ~ N M R}\left(\mathrm{CDCl}_{3}, 100 \mathrm{MHz}\right) \delta(\mathrm{ppm})$ (major diastereoisomer): 174.9, 83.2, 49.4, 44.2, 36.4, 29.1, 28.3, 26.8, 26.8, 24.9; HRMS (ESI) mass calculated for $[\mathrm{M}+\mathrm{H}]^{+}$ $\left(\mathrm{C}_{18} \mathrm{H}_{32} \mathrm{NO}_{3} \mathrm{~B}^{+}\right)$requires $m / z$ 322.2548, found $m / z$ 322.2563. 
2-((1s,3s)-1-cyclohexyl-3-((phenylsulfonyl)methyl)cyclobutyl)-4,4,5,5-tetramethyl-1,3,2dioxaborolane $(6 \mathrm{t})$

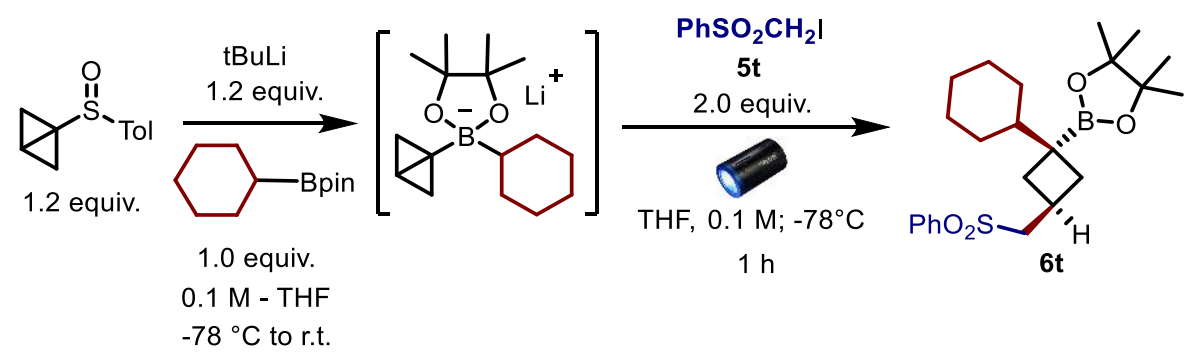

According to the General Procedure, using cyclohexyl boronic acid pinacol ester $(45 \mu \mathrm{L}$, $0.20 \mathrm{mmol}, 1.0$ equiv.) and iodomethyl phenylsulfone (112.8 $\mathrm{mg}, 0.40 \mathrm{mmol}, 2.0$ equiv.). The crude d.r. was measured to be $8.4: 1$ by ${ }^{1} \mathrm{H}-\mathrm{NMR}$ analysis. The product was purified by FCC (gradient hexane/ethyl acetate $=100: 0-90: 10)$ to afford the title compound $6 \mathbf{t}$ as a white solid in $69 \%$ yield $(58 \mathrm{mg})$; d.r. of the purified product $8.4: 1$.

MP: $141-143{ }^{\circ} \mathrm{C}$ (n-hexane/EtOAc); $\mathbf{R}_{\mathbf{f}}$ (70:30 $n$-hexane/ethyl acetate): 0.6; IR (film) $v_{\max } / \mathrm{cm}^{-1}: 2922,2850,1446,1381,1305,1140,747,688 ;{ }^{1} \mathbf{H} \mathbf{~ N M R}\left(\mathrm{CDCl}_{3}, 400 \mathrm{MHz}\right) \delta(\mathrm{ppm})$ (major diastereoisomer): $7.91-7.84(\mathrm{~m}, 2 \mathrm{H}), 7.66-7.59(\mathrm{~m}, 1 \mathrm{H}), 7.57-7.50(\mathrm{~m}, 2 \mathrm{H}), 3.13$ $(\mathrm{d}, \mathrm{J}=7.2 \mathrm{~Hz}, 2 \mathrm{H}), 2.40$ (hept, $J=7.2 \mathrm{~Hz}, 1 \mathrm{H}), 2.27-2.13(\mathrm{~m}, 2 \mathrm{H}), 1.72-1.49$ (m, 5H), 1.45 - $1.35(\mathrm{~m}, 2 \mathrm{H}), 1.27-0.97(\mathrm{~m}, 16 \mathrm{H}), 0.92-0.77(\mathrm{~m}, 2 \mathrm{H}) ;{ }^{13} \mathbf{C} \mathbf{~ N M R}\left(\mathrm{CDCl}_{3}, 100 \mathrm{MHz}\right)$ $\delta$ (ppm) (major diastereoisomer): 139.7, 133.6, 129.2, 128.2, 83.2, 62.8, 49.1, 36.3, 28.9, 26.8, 26.7, 25.7, 24.8; HRMS (ESI) mass calculated for $[\mathrm{M}+\mathrm{H}]^{+}\left(\mathrm{C}_{23} \mathrm{H}_{35} \mathrm{O}_{4} \mathrm{BS}^{+}\right)$requires $\mathrm{m} / \mathrm{z}$ 419.2422, found $m / z 419.2426$.

2-((1S,3s)-1-cyclohexyl-3-((R)-((4-fluorophenyl)sulfonyl)(phenyl)methyl)cyclobutyl)-4,4, 5,5-tetramethyl-1,3,2-dioxaborolane (6w)

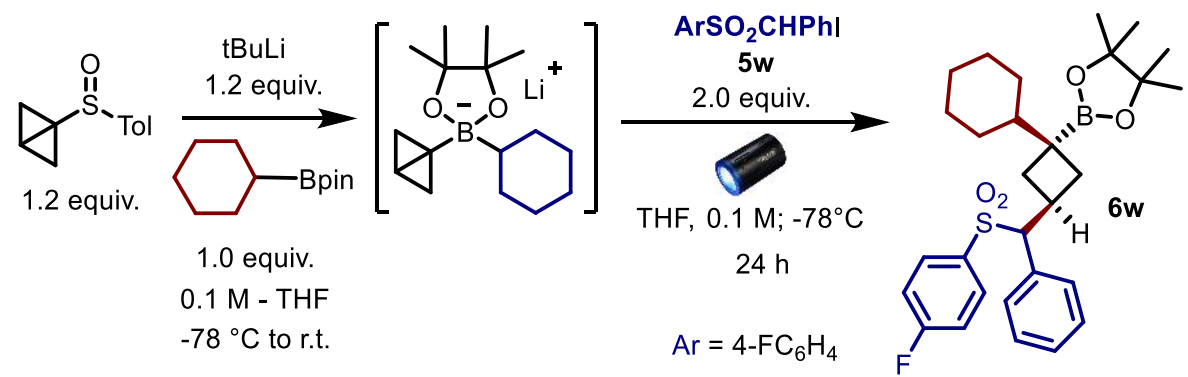

According to the General Procedure, using cyclohexyl boronic acid pinacol ester $(45 \mu \mathrm{L}$, $0.20 \mathrm{mmol}, 1.0$ equiv.) and iodobenzyl 4-fluorophenylsulfone $\mathbf{5 w}$ (150.4 $\mathrm{mg}, 0.40 \mathrm{mmol}, 2.0$ equiv.) for $24 \mathrm{~h}$. The crude d.r. was measured to be 3:1 by ${ }^{1} \mathrm{H}-\mathrm{NMR}$ analysis. The product was purified by FCC (gradient toluene/ethyl acetate $=99: 1-98: 2$ ) to afford the title compound $\mathbf{6 w}$ as a colourless oil in $83 \%$ yield $(85 \mathrm{mg})$; d.r. of the purified product $3: 1$. 
$\mathbf{R}_{\mathbf{f}}$ (80:20 $n$-hexane/ethyl acetate): 0.4; IR (film) $v_{\max } / \mathrm{cm}^{-1}: 2924,2851,1493,1312,1289$, 1141, 1083, 839, 697; ${ }^{1} \mathbf{H}$ NMR $\left(\mathrm{CDCl}_{3}, 500 \mathrm{MHz}\right) \delta$ (ppm) (major diastereoisomer): 7.52 7.44 (m, 2H), $7.26-7.16(\mathrm{~m}, 3 \mathrm{H}), 7.05-6.96(\mathrm{~m}, 4 \mathrm{H}), 4.28$ (d, J=11.4 Hz, 1H), $3.36-3.26$ (m, 1H), $2.38-2.20(\mathrm{~m}, 2 \mathrm{H}), 1.88-1.53(\mathrm{~m}, 7 \mathrm{H}), 1.53-1.37(\mathrm{~m}, 1 \mathrm{H}), 1.22(\mathrm{~s}, 12 \mathrm{H}), 1.16-$ $0.78(\mathrm{~m}, 5 \mathrm{H}) . ;{ }^{13} \mathbf{C ~ N M R}\left(\mathrm{CDCl}_{3}, 125 \mathrm{MHz}\right) \delta(\mathrm{ppm})$ (major diastereoisomer): 165.7 (d, $J=$ $256.6 \mathrm{~Hz}), 134.4$ (d, $J=3.1 \mathrm{~Hz}), 132.34,131.9$ (d, $J=9.8 \mathrm{~Hz}), 130.1(\mathrm{bp}), 128.7,128.4,115.9$ $(\mathrm{d}, J=22.4 \mathrm{~Hz}), 83.1,77.0,45.1,33.3,31.7,30.1,29.2,29.1,27.0,26.8,24.9,24.9$; HRMS (ESI) mass calculated for $[\mathrm{M}+\mathrm{H}]^{+}\left(\mathrm{C}_{23} \mathrm{H}_{35} \mathrm{O}_{4} \mathrm{BS}^{+}\right)$requires $m / z$ 419.2422, found $m / z$ 419.2426.

\section{Methyl (2-((1s,3R)-3-cyclohexyl-3-(4,4,5,5-tetramethyl-1,3,2-dioxaborolan-2-yl)cyclobut} yl)acetyl)-L-alanyl-L-phenylalaninate (6u)

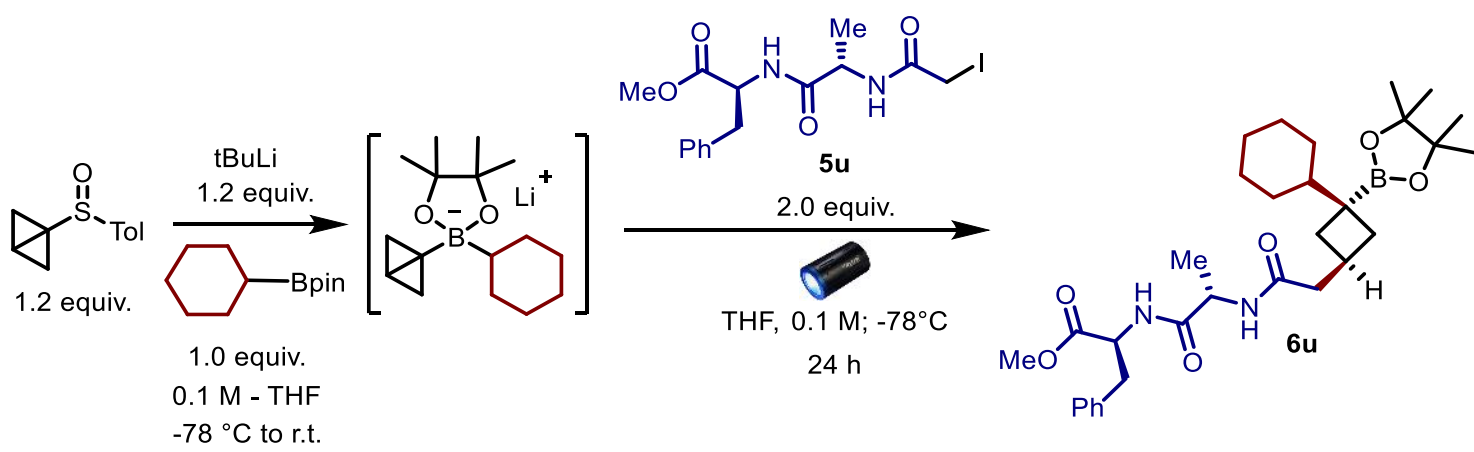

According to the General Procedure, using cyclohexyl boronic acid pinacol ester $(45 \mu \mathrm{L}$, $0.20 \mathrm{mmol}, 1.0$ equiv.) and iodoacetopeptide $5 \mathbf{u}(167 \mathrm{mg}, 0.40 \mathrm{mmol}, 2.0$ equiv.) for $24 \mathrm{~h}$. The crude d.r. of the product was measured by ${ }^{1} \mathrm{H}-\mathrm{NMR}$ and found to be $6.7: 1$. The crude product was purified by FCC (toluene:ethyl acetate $=80: 20$, after the elution of the sulfoxide byproduct the eluent was changed to hexane:acetone 80:20 to elute the desired product) to afford the title compound $6 \mathbf{u}$ as a white solid in $60 \%$ yield $(67 \mathrm{mg}), 6.7: 1$ d.r.

$[\boldsymbol{\alpha}]_{\mathbf{D}}{ }^{25}=+8.6(\mathrm{c}=1.0)$ MP: $48-50{ }^{\circ} \mathrm{C}\left(n\right.$-hexane/EtOAc); $\mathbf{R}_{\mathbf{f}}(40: 60 n$-hexane/ethyl acetate): 0.4; IR (film) $v_{\max } / \mathrm{cm}^{-1}: 3285,2924,2850,1748,1639,1544,1384,1143,966,700 ;{ }^{1} \mathbf{H}$ NMR $\left(\mathrm{CDCl}_{3}, 400 \mathrm{MHz}\right) \delta(\mathrm{ppm})$ (major diastereoisomer): $7.31-7.19(\mathrm{~m}, 3 \mathrm{H}), 7.13-7.05(\mathrm{~m}, 2 \mathrm{H})$, 6.57 (bs, 1H), 5.93 (bs, 1H), 4.80 (q, $J=6.5 \mathrm{~Hz}, 1 \mathrm{H}), 4.43$ (p, $J=7.0 \mathrm{~Hz}, 1 \mathrm{H}), 3.70$ (s, 3H), $3.13(\mathrm{dd}, \mathrm{J}=13.9,5.8 \mathrm{~Hz}, 1 \mathrm{H}), 3.05(\mathrm{dd}, J=13.9,6.5 \mathrm{~Hz}, 1 \mathrm{H}), 2.47-2.25$ (m, 3H), 2.26 $2.10(\mathrm{~m}, 2 \mathrm{H}), 1.75-1.55(\mathrm{~m}, 5 \mathrm{H}), 1.47-1.37(\mathrm{~m}, 2 \mathrm{H}), 1.30(\mathrm{~d}, J=6.5 \mathrm{~Hz}, 3 \mathrm{H}), 1.27-1.20$ $(\mathrm{m}, 13 \mathrm{H}), 1.20-1.02(\mathrm{~m}, 4 \mathrm{H}), 0.97-0.79(\mathrm{~m}, 2 \mathrm{H}) ;{ }^{13} \mathbf{C ~ N M R}\left(\mathrm{CDCl}_{3}, 100 \mathrm{MHz}\right) \delta(\mathrm{ppm})$ (major diastereoisomer): 172.0 (x2), 171.7, 135.8, 129.3, 128.7, 127.3, 83.2, 53.4, 52.5, 49.5, 48.6, 44.6, 37.9, 36.4, 29.1, 28.3, 26.9, 26.8, 24.9, 18.3; HRMS (ESI) mass calculated for $[\mathrm{M}+\mathrm{Na}]^{+}\left(\mathrm{C}_{31} \mathrm{H}_{47} \mathrm{~N}_{2} \mathrm{O}_{6} \mathrm{~B}^{+}\right)$requires $m / z$ 555.3600, found $m / z 355.3620$. 
(2aS,4S,5' R,6aS,6bS,8aS,8bR,9S,10R,11aS,12aS,12bR)-5',6a,8a,9-tetramethyldocosahyd rospiro[naphtho[2',1':4,5]indeno[2,1-b]furan-10,2'-pyran]-4-yl 2-((1s,3R)-3-cyclohexyl3$(4,4,5,5$-tetramethyl-1,3,2-dioxaborolan-2-yl)cyclobutyl)acetate (6v)

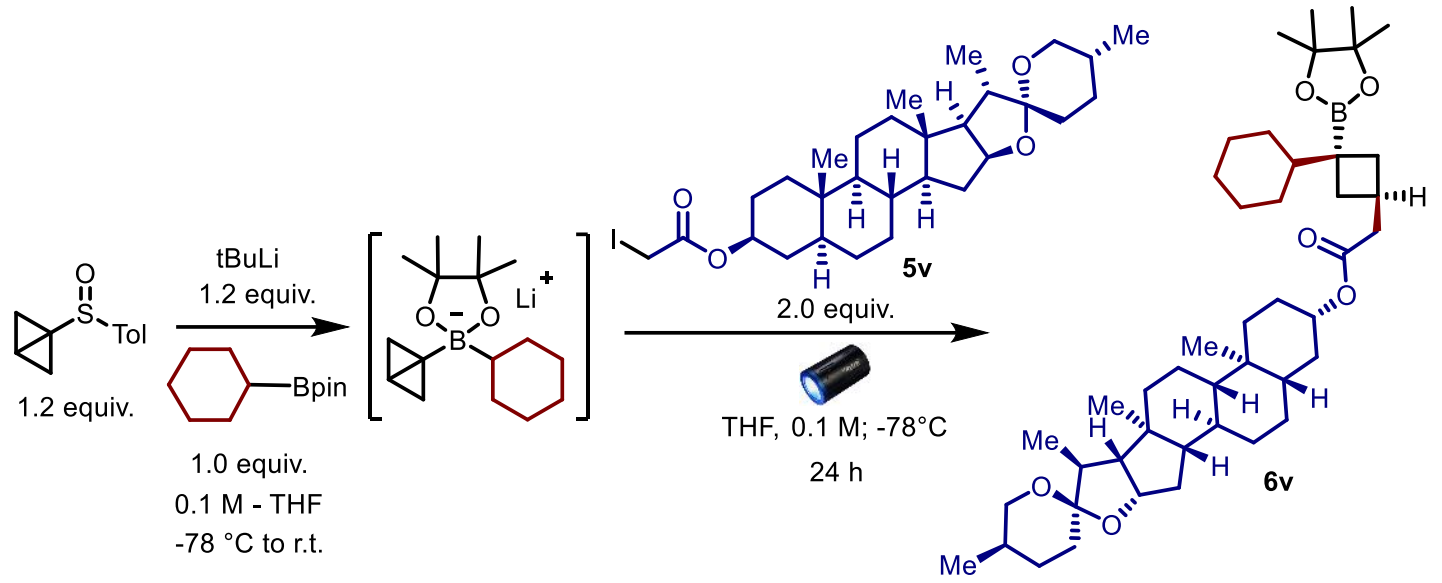

According to the General Procedure, using cyclohexyl boronic acid pinacol ester $(45 \mu \mathrm{L}$, $0.20 \mathrm{mmol}, 1.0$ equiv.) and alkyl iodide $5 \mathrm{v}$ (239.2 $\mathrm{mg}, 0.40 \mathrm{mmol}, 2.0$ equiv.) for $24 \mathrm{~h}$. The crude d.r. of the product was measured by ${ }^{1} \mathrm{H}-\mathrm{NMR}$ and found to be $5: 1$. The crude product was purified by FCC (gradient toluene:ethyl acetate $=99: 1-98: 2$ ) to afford the title compound 6v as a white solid in 78\% yield (110 mg), 5:1 d.r.

$[\boldsymbol{\alpha}]_{\mathbf{D}}{ }^{25}=-30.9(\mathrm{c}=1.4)$ MP: $65-66{ }^{\circ} \mathrm{C}$ ( $n$-hexane); $\mathbf{R}_{\mathbf{f}}(95: 5$ toluene/ethyl acetate): 0.4 ; IR (film) $v_{\max } / \mathrm{cm}^{-1}:$ 2924, 2849, 1731, 1450, 1379, 1307, 1240, 1174, 1144, 1053, 981; ${ }^{1} \mathbf{H}$ NMR $\left(\mathrm{CDCl}_{3}, 500 \mathrm{MHz}\right) \delta(\mathrm{ppm})$ (major diastereoisomer): $4.73-4.56(\mathrm{~m}, 1 \mathrm{H}), 4.37(\mathrm{q}, J=7.5 \mathrm{~Hz}$, $1 \mathrm{H}), 3.51-3.41(\mathrm{~m}, 1 \mathrm{H}), 3.36(\mathrm{t}, J=10.9 \mathrm{~Hz}, 1 \mathrm{H}), 2.45-2.35(\mathrm{~m}, 1 \mathrm{H}), 2.32-2.23(\mathrm{~m}, 3 \mathrm{H})$, $2.08-1.90(\mathrm{~m}, 1 \mathrm{H}), 1.90-0.68(\mathrm{~m}, 63 \mathrm{H}), 0.69-0.61(\mathrm{~m}, 1 \mathrm{H}) ;{ }^{13} \mathbf{C} \mathbf{N M R}\left(\mathrm{CDCl}_{3}, 125 \mathrm{MHz}\right)$ $\delta(\mathrm{ppm})$ (major diastereoisomer): 172.5, 109.3, 83.1, 81.0, 73.4, 67.0, 62.3, 56.4, 54.3, 49.4, $44.8,42.5,41.8,40.7,40.1,36.9,36.3,36.3,35.7,35.2,34.2,32.3,31.9,31.5,30.4,29.2$, 28.9, 28.6, 28.3 27.7, 26.9, 26.9, 24.9, 21.1, 17.3, 16.6, 14.6, 12.4; HRMS (ESI) mass calculated for $[\mathrm{M}+\mathrm{H}]^{+}\left(\mathrm{C}_{45} \mathrm{H}_{73} \mathrm{O}_{6} \mathrm{~B}^{+}\right)$requires $m / z$. 721.5573, found $m / z$ 721.5571. 


\subsection{General Procedure for the Reductive Desulfonylation}

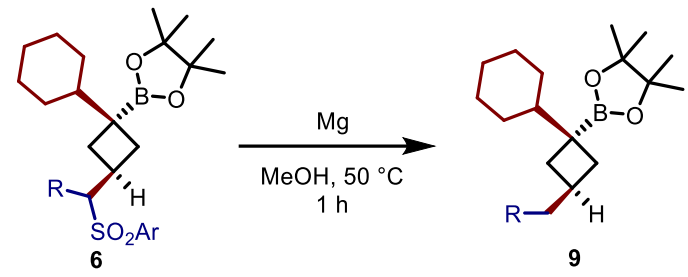

To a stirred solution of compounds $6(0.1 \mathrm{mmol})$ in dry methanol $(5.0 \mathrm{~mL})$ under $\mathrm{N}_{2}$ atmosphere was added freshly activated magnesium flakes ${ }^{\mathrm{A}}(97 \mathrm{mg}, 4.0 \mathrm{mmol})$ and the mixture was vigorously stirred at $50{ }^{\circ} \mathrm{C}$ for one hour. The reaction was then diluted with $50 \mathrm{~mL}$ of ammonium chloride saturated solution and extracted three times with pentane $(50 \mathrm{ml})$. The solvents were removed under vacuo and the residue was purified by flash column chromatography to afford the desired cyclobutyl boronic esters 9 .

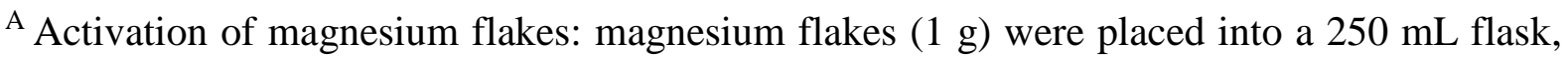
and $10 \mathrm{~mL}$ of dry diethyl ether were added. Few crystals of iodine were added to the mixture, the yellowish mixture was subjected to sonication (2-3 minutes) and vigorously stirred, gently heating with a heating gun until full decolouration occurred. The process was repeated twice, and the solvent was removed under vacuum. The flakes were used immediately avoiding prolonged exposure to air.

2-((1s,3s)-1-cyclohexyl-3-methylcyclobutyl)-4,4,5,5-tetramethyl-1,3,2-dioxaborolane (9a)
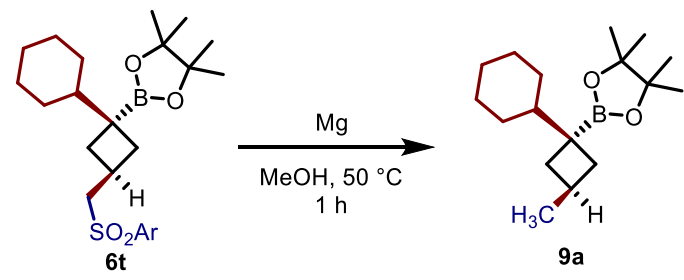

According to the General Procedure, using compound 6t. The crude d.r. of the product was measured by GC-MS and found to be 8:1. The crude product was purified by FCC (gradient hexane:dichloromethane $=98: 2-95: 5)$ to afford the title compound 9a as a colourless oil in $76 \%$ yield $(21 \mathrm{mg})$.

$\mathbf{R}_{\mathbf{f}}$ (95:5 toluene/ethyl acetate): 0.4; IR (film) $v_{\max } / \mathrm{cm}^{-1}: 2921,2850,1384,1296,1143,967$, 864; ${ }^{1} \mathbf{H}$ NMR $\left(\mathrm{CDCl}_{3}, 400 \mathrm{MHz}\right) \delta$ (ppm) (major diastereoisomer): $2.31-2.18(\mathrm{~m}, 2 \mathrm{H}), 2.15$ - $2.03(\mathrm{~m}, 1 \mathrm{H}), 1.79-1.56(\mathrm{~m}, 5 \mathrm{H}), 1.34-1.28(\mathrm{~m}, 2 \mathrm{H}), 1.26(\mathrm{~s}, 12 \mathrm{H}), 1.24-1.07(\mathrm{~m}, 4 \mathrm{H})$, $0.96(\mathrm{~d}, J=6.5 \mathrm{~Hz}, 3 \mathrm{H}), 0.93-0.86(\mathrm{~m}, 2 \mathrm{H}) ;{ }^{13} \mathbf{C ~ N M R}\left(\mathrm{CDCl}_{3}, 100 \mathrm{MHz}\right) \delta(\mathrm{ppm})$ (major diastereoisomer): 83.0, 49.4, 38.3, 29.3, 27.0, 27.0, 26.7, 24.9, 22.7; HRMS (APCI) mass calculated for $[\mathrm{M}+\mathrm{H}]^{+}\left(\mathrm{C}_{17} \mathrm{H}_{31} \mathrm{O}_{2} \mathrm{~B}^{+}\right)$requires $m / z$ 279.2490, found $m / z$ 279.2488. 

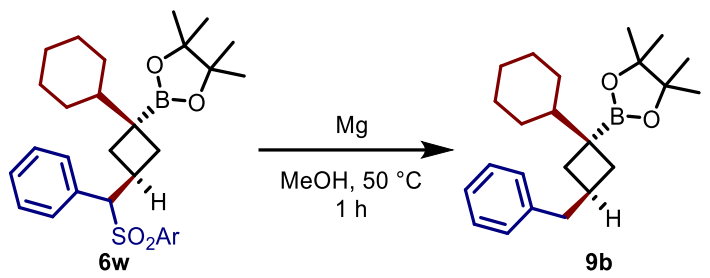

According to the General Procedure, using compound 6w. The crude d.r. of the product was measured by GC-MS and found to be 3:1. The crude product was purified by FCC (gradient pentane:ethyl acetate $=98: 2$ ) to afford the title compound $\mathbf{9 b}$ as a colourless oil in $96 \%$ yield (33 mg).

$\mathbf{R}_{\mathbf{f}}$ (95:5 toluene/ethyl acetate): 0.4; IR (film) $v_{\max } / \mathrm{cm}^{-1}: 2921,2849,1383,1296,1141,860$, 698; ${ }^{1} \mathbf{H}$ NMR $\left(\mathrm{CDCl}_{3}, 400 \mathrm{MHz}\right) \delta$ (ppm) (major diastereoisomer): 7.30 - 7.21 (m, 2H), 7.20 $-7.09(\mathrm{~m}, 3 \mathrm{H}), 2.71(\mathrm{~d}, J=7.9 \mathrm{~Hz}, 2 \mathrm{H}), 2.52-2.38(\mathrm{~m}, 1 \mathrm{H}), 1.98-1.83(\mathrm{~m}, 4 \mathrm{H}), 1.76-1.60$ $(\mathrm{m}, 5 \mathrm{H}), 1.49-1.35(\mathrm{~m}, 1 \mathrm{H}), 1.33-1.04(\mathrm{~m}, 15 \mathrm{H}), 1.00-0.84(\mathrm{~m}, 2 \mathrm{H}) ;{ }^{13} \mathbf{C ~ N M R}\left(\mathrm{CDCl}_{3}\right.$, $100 \mathrm{MHz}) \delta(\mathrm{ppm})$ (major diastereoisomer): 141.7, 128.8, 128.3, 125.7, 83.1, 47.1, 43.0, 33.4, 32.3, 29.4, 27.1, 27.0, 25.0; HRMS (ESI) mass calculated for $[\mathrm{M}+\mathrm{H}]^{+}\left(\mathrm{C}_{23} \mathrm{H}_{31} \mathrm{O}_{2} \mathrm{~B}^{+}\right)$requires $m / z, 377.2622$, found $m / z 377.2627$.

\subsection{Product Functionalization Reactions}

\subsubsection{Oxidation to Alcohol}
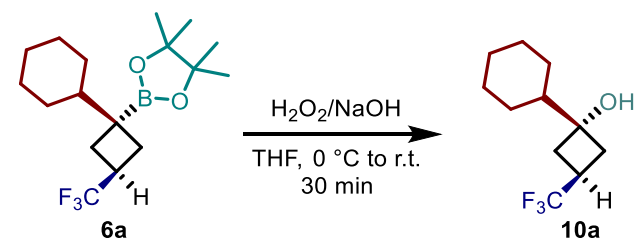

Boronic ester 6a $(33.2 \mathrm{mg}, 0.1 \mathrm{mmol})$ was placed in a test tube and dissolved in THF (1 mL). The solution was cooled down to $0{ }^{\circ} \mathrm{C}$ and a $2: 1 \mathrm{NaOH}(2 \mathrm{M}): \mathrm{H}_{2} \mathrm{O}_{2}(33 \%$ aq.) mixture $(1 \mathrm{~mL})$ was added dropwise. The reaction was stirred 10 minutes at $0{ }^{\circ} \mathrm{C}$, followed by 20 minutes at room temperature. The reaction was dissolved in diethyl ether $(100 \mathrm{~mL})$ and washed with $\mathrm{NH}_{4} \mathrm{Cl}$ saturated solution $(50 \mathrm{~mL})$. The aqueous phase was extracted twice with $50 \mathrm{~mL}$ of diethyl ether. The combined organic phase was washed with brine $(50 \mathrm{~mL})$, dried over $\mathrm{MgSO}_{4}$ and evaporated to afford the crude alcohol, which was purified by FCC (gradient $n$ pentane:diethyl ether $=85: 15-70: 30$ ) to afford the title compound 10a as a colourless oil in $95 \%$ yield $(21 \mathrm{mg})$. 
Rf (60:40 $n$-pentane:diethyl ether): 0.5; IR (film) $v_{\max } / \mathrm{cm}^{-1}: 3409,2987,2855,1451,1387$, 1270, 1149, 1094, 970, 919, 725; ${ }^{1} \mathbf{H} \mathbf{~ N M R}\left(\mathrm{CDCl}_{3}, 500 \mathrm{MHz}\right) \delta(\mathrm{ppm}): 3.18-3.01(\mathrm{~m}, 1 \mathrm{H})$, $2.24-2.13(\mathrm{~m}, 2 \mathrm{H}), 2.10-2.00(\mathrm{~m}, 2 \mathrm{H}), 1.86-1.75(\mathrm{~m}, 2 \mathrm{H}), 1.74-1.60(\mathrm{~m}, 3 \mathrm{H}), 1.48(\mathrm{~s}$, 1H), 1.35 (tt, $J=12.1,3.2 \mathrm{~Hz}, 1 \mathrm{H}), 1.29-1.06$ (m, 3H), 0.98 (qd, $J=12.5,3.4 \mathrm{~Hz}, 2 \mathrm{H})$; ${ }^{13} \mathrm{C} \mathrm{NMR}\left(\mathrm{CDCl}_{3}, 125 \mathrm{MHz}\right) \delta(\mathrm{ppm}): 127.8(\mathrm{q}, J=277 \mathrm{~Hz}), 76.06,47.4,33.3(\mathrm{q}, J=3.6 \mathrm{~Hz})$, $30.8(\mathrm{q}, J=31 \mathrm{~Hz}), 26.4,26.2,25.3 ;{ }^{19} \mathbf{F ~ N M R}\left(\mathrm{CDCl}_{3}, 376 \mathrm{MHz}\right) \delta(\mathrm{ppm}):-73.4(\mathrm{~d}, J=9.2$ $\mathrm{Hz})$. HRMS (APCI) mass calculated for $[\mathrm{M}+\mathrm{H}]^{+}\left(\mathrm{C}_{11} \mathrm{H}_{17} \mathrm{O}_{2} \mathrm{~F}_{3}{ }^{+}\right)$requires $m / z$ 223.1304, found $m / z 223.1297$.

\subsubsection{Synthesis of Potassium Trifluoroborate Salt}

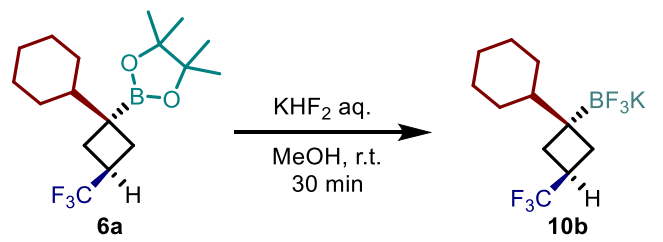

According to a literature procedure, ${ }^{4}$ boronic ester $\mathbf{6 a}(33.2 \mathrm{mg}, 0.1 \mathrm{mmol})$ was dissolved in $\mathrm{MeOH}(1.7 \mathrm{~mL})$ and an aqueous solution of $\mathrm{KHF}_{2}(4.5 \mathrm{M}, 130 \mu \mathrm{L})$ was added dropwise. Solvents were evaporated and the residue was redissolved in a 3:2 mixture $\mathrm{MeOH}: \mathrm{H}_{2} \mathrm{O}(10$ $\mathrm{mL}$ ), followed by evaporation. The cycle solubilization - evaporation was repeated 10 times, to remove all the pinacol byproduct. The residue was triturated with diethyl ether $(20 \mathrm{~mL})$, collecting the liquid phase and the process repeated twice. The combined organic phases were filtered to remove solid particles and evaporated to afford the title compound $\mathbf{1 0 b}$ as a white solid in $77 \%$ yield $(24 \mathrm{mg})$.

MP : $230{ }^{\circ} \mathrm{C}$ (decomp.); IR (film) $v_{\max } / \mathrm{cm}^{-1}: 2929,2853,1385,1285,1171,1145,1124,1082$ 995, 945, 888; ${ }^{1} \mathbf{H}$ NMR (CD $\left.3 \mathrm{CN}, 500 \mathrm{MHz}\right) \delta(\mathrm{ppm}): 3.00-2.79(\mathrm{~m}, 1 \mathrm{H}), 1.99-1.94(\mathrm{~m}$, $2 \mathrm{H}), 1.72-1.64(\mathrm{~m}, 2 \mathrm{H}), 1.64-1.45(\mathrm{~m}, 5 \mathrm{H}), 1.18-1.00(\mathrm{~m}, 6 \mathrm{H}) ;{ }^{13} \mathrm{C} \mathrm{NMR}\left(\mathrm{CD}_{3} \mathrm{CN}\right.$, $125 \mathrm{MHz}) \delta(\mathrm{ppm}): 129.6(\mathrm{q}, J=274.9 \mathrm{~Hz}), 51.8,33.2(\mathrm{q}, J=29 \mathrm{~Hz}), 29.5,29.0,28.4,27.9$; ${ }^{19} \mathbf{F}$ NMR $\left(\mathrm{CD}_{3} \mathrm{CN}, 376 \mathrm{MHz}\right) \delta(\mathrm{ppm}):-73.76(\mathrm{~d}, \mathrm{~J}=10.3 \mathrm{~Hz}, 3 \mathrm{~F}),-145.16$ (bs, 3F). ${ }^{11} \mathbf{B}$ NMR $\left(\mathrm{CD}_{3} \mathrm{CN}, 128 \mathrm{MHz}\right) \delta 4.3(\mathrm{q}, J=64.1 \mathrm{~Hz})$. HRMS (ESI) mass calculated for $[\mathrm{M}-\mathrm{K}]^{-}$ $\left(\mathrm{C}_{11} \mathrm{H}_{16} \mathrm{~F}_{6} \mathrm{~B}^{-}\right)$requires $m / z$ 273.1249, found $m / z$ 273.1261. 


\subsubsection{Zweifel Olefination}
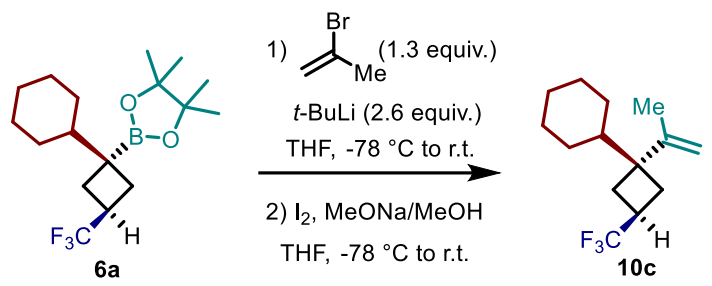

Freshly distilled 2-bromopropene $(0.13 \mathrm{mmol}, 11.6 \mu \mathrm{L})$ was dissolved in THF $(1 \mathrm{~mL})$. The solution was cooled down to $-78{ }^{\circ} \mathrm{C}$ and a freshly titrated pentane tert-butyllithium solution (0.26 mmol) was added dropwise. After stirring at $-78{ }^{\circ} \mathrm{C}$ for $1 \mathrm{~h}$, a solution of boronic ester 6a (33.2 $\mathrm{mg}, 0.1 \mathrm{mmol}$ in $0.7 \mathrm{~mL}$ of THF) was added dropwise at the same temperature. The reaction was stirred 5 minutes at $-78{ }^{\circ} \mathrm{C}$, then warmed up to room temperature and stirred for $1 \mathrm{~h}$. The reaction was cooled down to $-78{ }^{\circ} \mathrm{C}$ and a solution of iodine ( $32.9 \mathrm{mg}$ in $0.7 \mathrm{~mL}$ THF) was added dropwise. The solution was stirred 15 minutes at $-78{ }^{\circ} \mathrm{C}$, followed by further 15 minutes at room temperature. The solution was cooled down again to $-78{ }^{\circ} \mathrm{C}$ and $\mathrm{NaOMe}(16$ $\mathrm{mg}, 0.3 \mathrm{mmol}$ ) was added under nitrogen flow, followed by anhydrous methanol (1 mL). The reaction was stirred 10 minutes at $-78{ }^{\circ} \mathrm{C}$ followed by $1 \mathrm{~h}$ at room temperature. The reaction was diluted with pentane $(150 \mathrm{~mL})$, washed with a saturated $\mathrm{Na}_{2} \mathrm{~S}_{2} \mathrm{O}_{3}$ solution $(50 \mathrm{~mL})$ and brine $(50 \mathrm{~mL})$. The organic phase was dried over magnesium sulfate and solvents evaporated. The residue was passed through a Pasteur/silica plug $(6.5 \mathrm{~cm})$ using pure pentane as eluent. Solvents were evaporated to afford the title compound 10c as a colourless oil in $93 \%$ yield (23 $\mathrm{mg})$.

Rf: (n-pentane): 0.9; IR (film) $v_{\max } / \mathrm{cm}^{-1}: 2926,2854,1385,1637,1449,1389,1275,1148$, 1086, 1007, 890; ${ }^{1} \mathbf{H}$ NMR $\left(\mathrm{CDCl}_{3}, 500 \mathrm{MHz}\right) \delta(\mathrm{ppm}): \delta 5.04(\mathrm{p}, J=1.4 \mathrm{~Hz}, 1 \mathrm{H}), 4.95-4.79$ $(\mathrm{m}, 1 \mathrm{H}), 2.77-2.62(\mathrm{~m}, 1 \mathrm{H}), 2.18-2.10(\mathrm{~m}, 2 \mathrm{H}), 2.10-2.02(\mathrm{~m}, 2 \mathrm{H}), 1.81-1.74(\mathrm{~m}, 2 \mathrm{H})$, $1.72(\mathrm{~d}, J=1.4 \mathrm{~Hz}, 3 \mathrm{H}), 1.69-1.56(\mathrm{~m}, 3 \mathrm{H}), 1.35(\mathrm{tt}, J=12.1,3.1 \mathrm{~Hz}, 1 \mathrm{H}), 1.23-1.02(\mathrm{~m}$, $3 \mathrm{H}), 0.86(\mathrm{qd}, J=12.6,3.3 \mathrm{~Hz}, 2 \mathrm{H}) ;{ }^{13} \mathbf{C} \mathbf{N M R}\left(\mathrm{CDCl}_{3}, 125 \mathrm{MHz}\right) \delta(\mathrm{ppm}): 147.0,127.7(\mathrm{q}, J$ $=275.8 \mathrm{~Hz}), 112.4,47.7,44.4,31.8$ (q, $J=31.6 \mathrm{~Hz}), 27.9$ (q, $J=3.1 \mathrm{~Hz}), 27.6,26.9$, 26.6, $20.1 ;{ }^{19} \mathbf{F}$ NMR $\left(\mathrm{CD}_{3} \mathrm{CN}, 376 \mathrm{MHz}\right) \delta(\mathrm{ppm}):-73.0(\mathrm{~d}, J=8.9 \mathrm{~Hz})$. HRMS (APCI) mass calculated for $[\mathrm{M}]^{+}\left(\mathrm{C}_{14} \mathrm{H}_{21} \mathrm{~F}_{3}{ }^{+}\right)$requires $m / z$ 246.1590, found $m / z$ 246.1584. 


\subsubsection{Matteson Homologation}
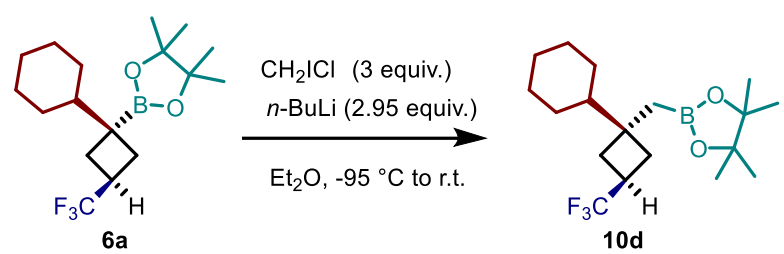

According to a literature procedure, ${ }^{9}$ boronic ester $6 \mathbf{6 a}(33.2 \mathrm{mg}, 0.1 \mathrm{mmol})$ and iodochloromethane $(21.9 \mu \mathrm{L}, 0.3 \mathrm{mmol})$ were dissolved in diethyl ether $(1 \mathrm{~mL})$ and cooled to $-95{ }^{\circ} \mathrm{C}$ (methanol/liquid nitrogen bath). $n$-butyllithium $(0.295 \mathrm{mmol}, 1.6 \mathrm{M}$ solution in $n$ hexane) was added dropwise and the solution was stirred 10 minutes at $-95{ }^{\circ} \mathrm{C}$, followed by additional $1 \mathrm{~h}$ at room temperature. The whole mixture was plugged through a thin layer of silica and eluted with $\mathrm{Et}_{2} \mathrm{O}$. Solvents were evaporated to afford the crude boronic ester, containing impurities of starting material and overhomologated product. The crude mixture was purified by FCC (gradient $n$-pentane:dichloromethane $=95: 5-85: 15$ ) to afford the title compound $10 \mathrm{~d}$ as a colourless oil in $66 \%$ yield $(23 \mathrm{mg})$.

$\mathbf{R}_{\mathbf{f}}\left(70: 30 n\right.$-hexane:dichloromethane): 0.25; IR (film) $v_{\max } / \mathrm{cm}^{-1}: 2977,2925,2854,1450,1358$, 1327, 1273, 1145, 1097, 1005, 967, 889, 850; ${ }^{1} \mathbf{H} \mathbf{~ N M R}\left(\mathrm{CDCl}_{3}, 400 \mathrm{MHz}\right) \delta(\mathrm{ppm}): 3.04-$ $2.85(\mathrm{~m}, 1 \mathrm{H}), 2.03-1.89(\mathrm{~m}, 4 \mathrm{H}), 1.81-1.70(\mathrm{~m}, 2 \mathrm{H}), 1.70-1.60(\mathrm{~m}, 1 \mathrm{H}), 1.59-1.50(\mathrm{~m}$, 2H), 1.25 (s, 16H), 0.93 (s, 2H), $0.92-0.82(\mathrm{~m}, 2 \mathrm{H}) ;{ }^{13} \mathbf{C ~ N M R}\left(\mathrm{CDCl}_{3}, 125 \mathrm{MHz}\right) \delta(\mathrm{ppm})$ : $128.2(\mathrm{q}, J=275.6 \mathrm{~Hz}), 83.1,49.6,38.9,31.8(\mathrm{q}, J=3.1 \mathrm{~Hz}), 30.7(\mathrm{q}, J=30.2 \mathrm{~Hz}), 26.9,26.8$, 26.8, 25.0; ${ }^{19} \mathbf{F ~ N M R}\left(\mathrm{CDCl}_{3}, 376 \mathrm{MHz}\right) \delta(\mathrm{ppm})$ : -73.2 (d, J = 9.7 Hz). HRMS (APCI) mass calculated for $[\mathrm{M}+\mathrm{H}]^{+}\left(\mathrm{C}_{18} \mathrm{H}_{30} \mathrm{O}_{2} \mathrm{~F}_{3} \mathrm{~B}^{+}\right)$requires $m / z$ 347.2364, found $m / z$ 347.2353. 


\section{Determination of the Stereochemistry}

The relative configuration of products 6 was unambiguously inferred by ${ }^{1} \mathrm{H}-\mathrm{NMR}$ NOE analysis. The results of our investigation are detailed below.

Investigation on the model compound 6 a:

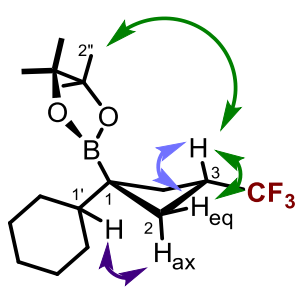

$6 a$

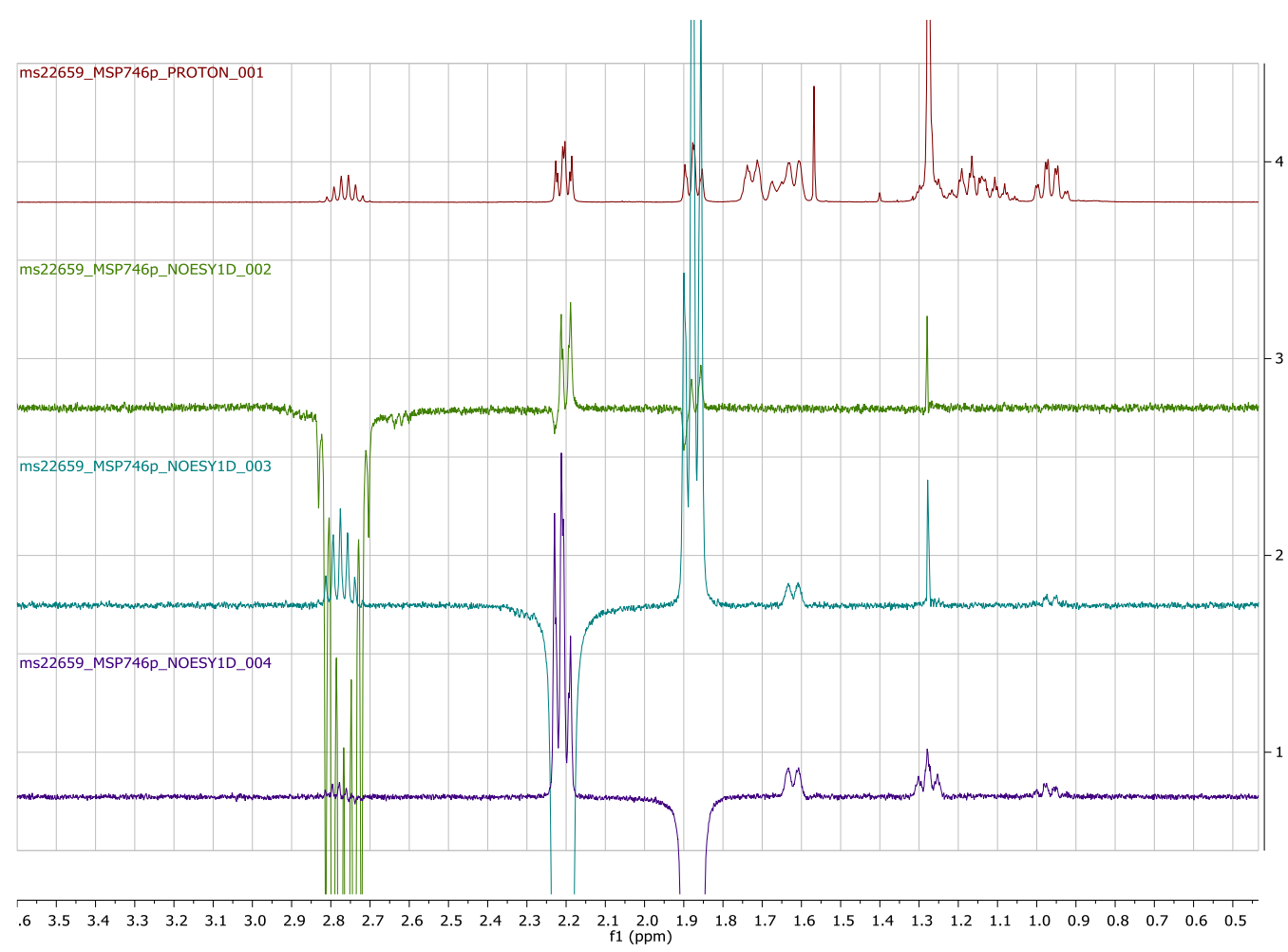

Figure S2. NOE study and stereochemistry inferred for compound $\mathbf{6 a}$.

Significant NOE correlations observed in compound 6a:

1. Irradiation of $\boldsymbol{H}-3(2.76 \mathrm{ppm})$ : NOE correlation was observed with H-2" (1.28 ppm) and with the equatorial $\mathrm{H}-2$ (2.21 ppm).

2. Irradiation of equatorial $\mathbf{H}-2(2.21 \mathrm{ppm})$ : NOE correlation was observed with $\mathrm{H}-3$ (2.76 ppm), with H-2" (1.28 ppm, not shown in the molecular structure for clarity) and with the axial H-2 (1.88 ppm).

3. Irradiation of axial $\mathbf{H}-2(\mathbf{1 . 8 8} \mathbf{~ p p m})$ : NOE correlation was observed with equatorial H-2 (2.21 ppm) and with H-1' (1.28 ppm). 
Comment on the NOE study of 6a: The NOE study described above, in particular the strong NOE correlation identified between H-3 and the pinacol H-2" proves the stereochemistry described in Fig. S2.

\section{Investigation on compound 6p:}

A further investigation was carried out on compound 6p. The aim of this second NOE investigation was to carry out a full study on the two opposite diastereoisomers of the product to further confirm the results obtained with the previous investigation. In order to obtain $\mathbf{6 p}$ as a mixture of diastereoisomers, the photochemical reaction was carried out following the procedure as presented in section 3.2, but using 1,3-dimethyl-2-imidazolidinone as solvent in the photochemical step, which was carried out at room temperature (6p obtained in $72 \%$ yield, 3.5:1 d.r.). Compound $\mathbf{6 p}$ was specifically used for this investigation because of the particular ease of purification of the two diastereoisomers, which ensures a reliable study.

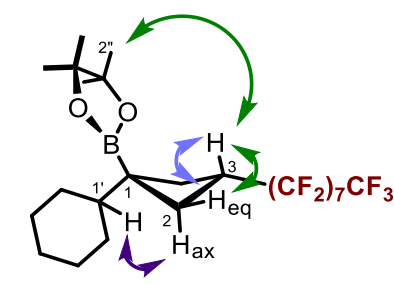

$6 p$ - major

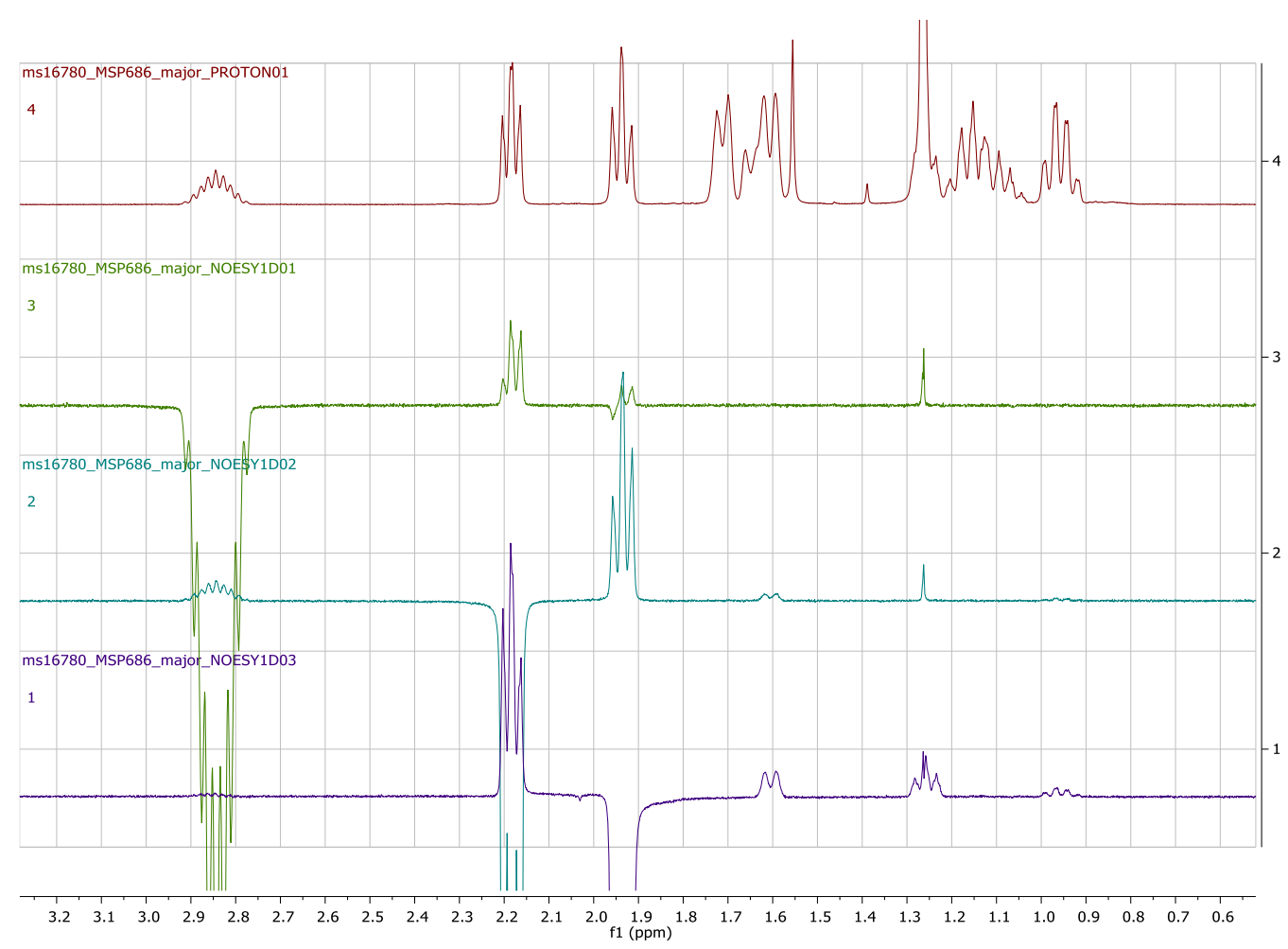

Figure S3. NOE study and stereochemistry inferred for compound $\mathbf{6 p}$ major. 
Significant on NOE correlations observed in compound 6p major:

1. Irradiation of $\boldsymbol{H}-3$ (2.84 ppm): NOE correlation was observed with H-2" (1.26 ppm) and with the equatorial $\mathrm{H}-2$ (2.18 ppm).

2. Irradiation of equatorial $\mathbf{H}-2(2.18 \mathrm{ppm})$ : NOE correlation was observed with $\mathrm{H}-3$ (2.84 ppm), with H-2" (1.26 ppm, not shown in the molecular structure for clarity) and with the axial H-2 (1.93 ppm).

3. Irradiation of axial $\mathbf{H}-2(1.93 \mathrm{ppm})$ : NOE correlation was observed with equatorial $\mathrm{H}-$ 2 (2.21 ppm) and with H-1' (1.26 ppm).
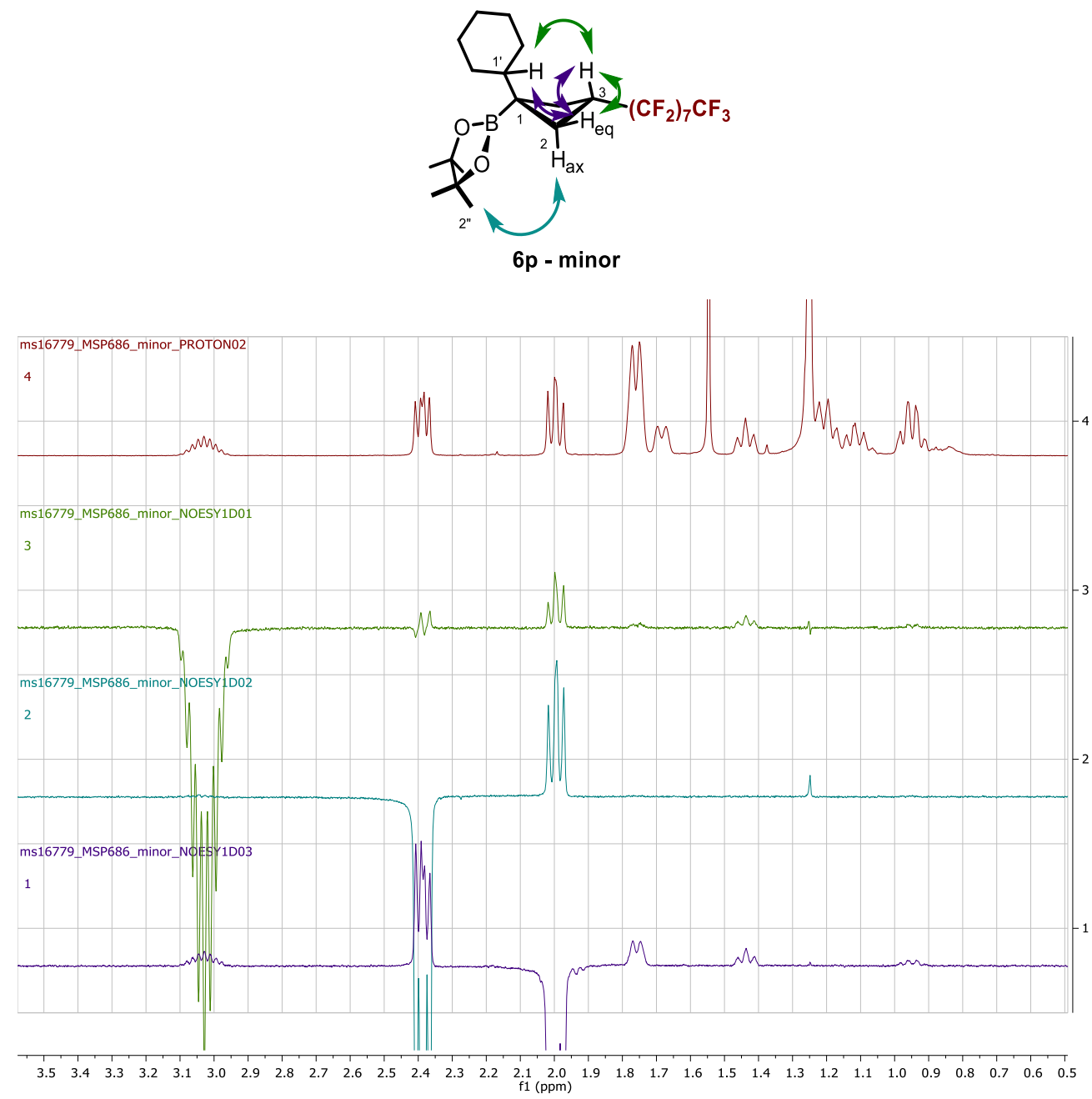

Figure S4. NOE study and stereochemistry inferred for compound $\mathbf{6 p}$ minor.

Significant NOE correlations observed in compound $\mathbf{6 p}$ minor:

1. Irradiation of $\boldsymbol{H}-3$ (3.02 ppm): NOE correlation was observed with H-1' (1.43 ppm) and with the equatorial $\mathrm{H}-2(2.00 \mathrm{ppm})$.

2. Irradiation of axial $\mathbf{H}-2$ (2.39 ppm): NOE correlation was observed with H-2" (1.25 ppm), and with equatorial H-2 (1.99 ppm). 
3. Irradiation of equatorial $\mathbf{H}-2$ (1.99 ppm): NOE correlation was observed with H-3 (3.02 ppm), with H-1' (1.43 pppm) and with the axial H-2 (2.39 ppm).

Comment on the combined NOE study of the two diastereoisomers of $6 p$ : The combined NOE study of the two diastereoisomers of compound $\mathbf{6 p}$ confirmed the expected stereochemistry. The most remarkable evidence is that a NOE correlation between $\mathrm{H}-3$ and the pinacol H-2" (and the absence of correlation with $\mathrm{H}^{-1} 1^{\prime}$ ) was observed in the major diastereoisomer, while a NOE correlation between H-3 and H-1' (and the absence of correlation with the H-2") was observed in the minor diastereoisomer.

\section{Investigation on the aryl substituted compound $6 \mathrm{~g}$ :}

As better detailed in section 5.2, we consider likely that aryl substituted BCB-boronates follow a mechanistically distinct pathway compared to their alkyl analogues, so a study was carried on $\mathbf{6 g}$ to determine the stereochemistry. $\mathbf{6 g}$ was specifically used for this investigation because samples enriched with the two diastereoisomers could be obtained, ensuring a reliable study.
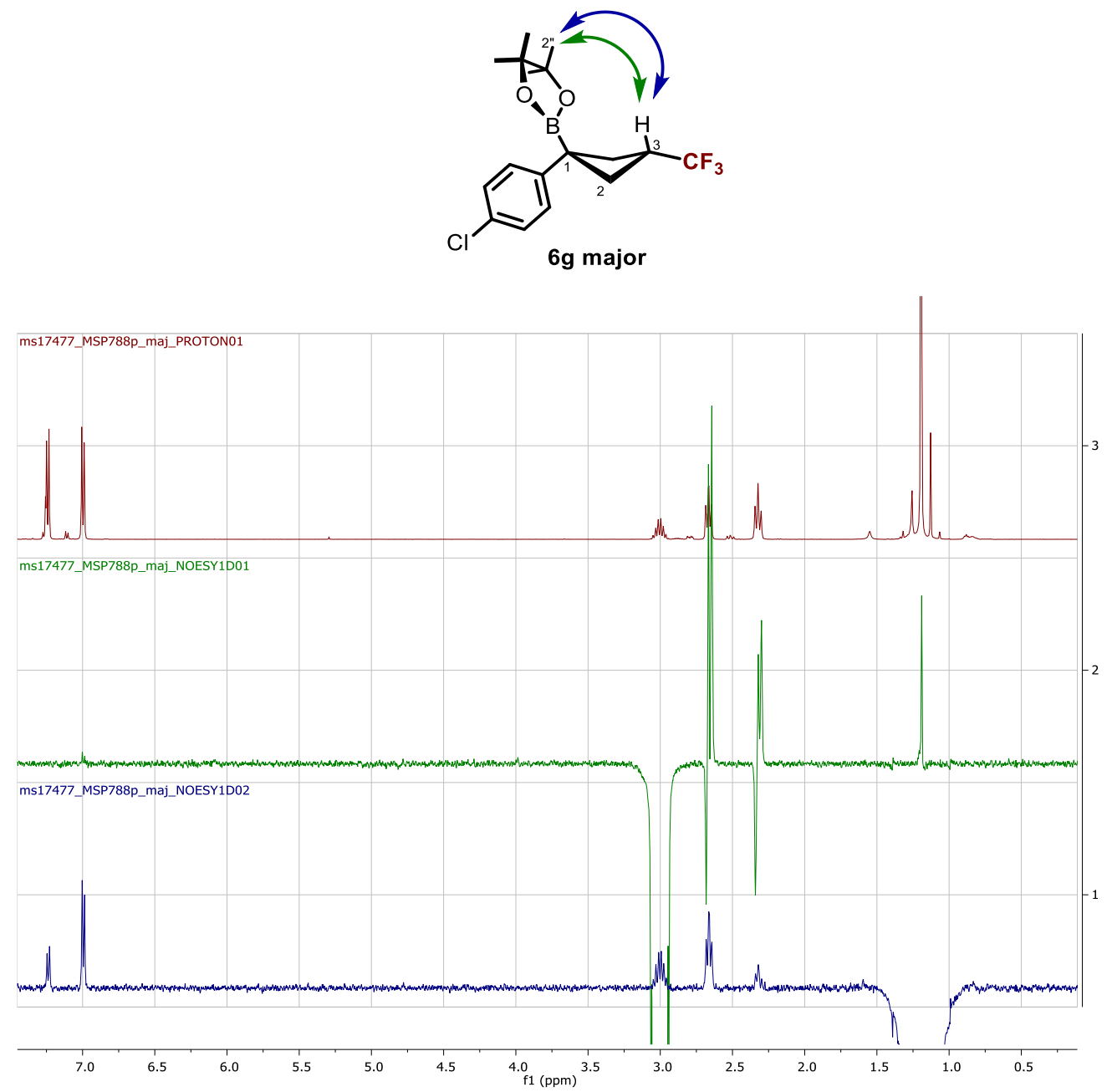

Figure S5. NOE study and stereochemistry inferred for compound $\mathbf{6 g}$ major. 
Significant NOE correlations observed in compound $6 \mathrm{~g}$ major:

1. Irradiation of $\boldsymbol{H}-3$ (3.00 ppm): NOE correlation was observed with H-2" (1.19 ppm).

2. Irradiation of $\boldsymbol{H - 2}$ "(1.19 ppm): NOE correlation was observed with H-3 (3.00 ppm), in consonance with the results obtained irradiating H-3.
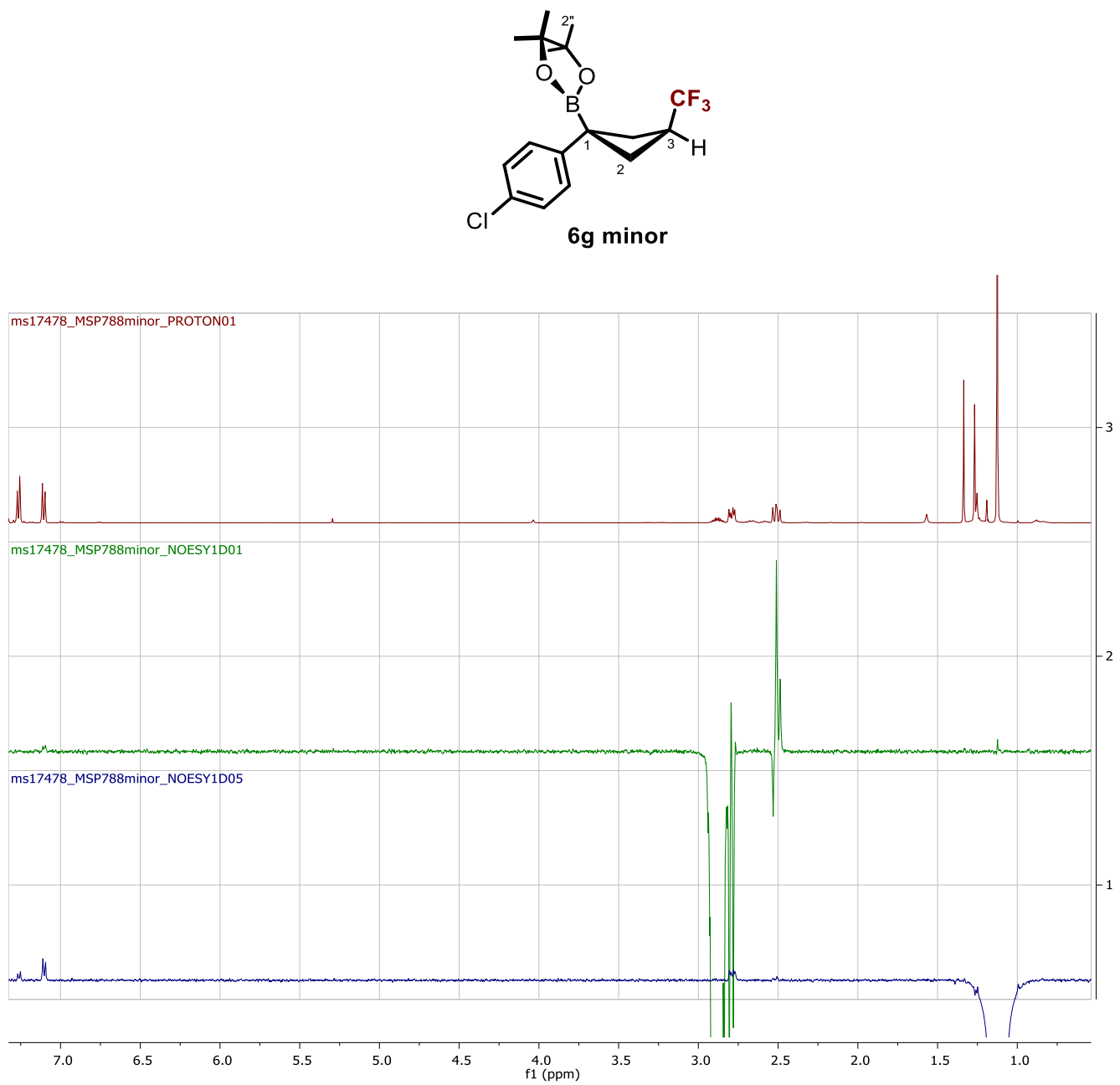

Figure S6. NOE study and stereochemistry inferred for compound $\mathbf{6 g}$ minor.

Analysis of the spectra of $\mathbf{6 g}$ minor:

1. Irradiation of $\mathbf{H}-3$ (2.87 ppm): Absence of significant correlation was observed with $\mathrm{H}-2$ " (1.12 ppm). The residual very weak NOE signal at $1.12 \mathrm{ppm}$ is due to partial undesired irradiation of the H-2 (m, $2.73-2.82)$, which partially overlaps with H-3.

2. Irradiation of $\mathbf{H - 2}$ "(1.12 ppm): Absence of detectable NOE correlation was observed with H-3 (2.87 ppm), in consonance with the results obtained irradiating H-3. 
Comment on the combined NOE study of the two diastereoisomers of $6 \mathrm{~g}$ : The combined NOE study of the two diastereoisomers of compound $\mathbf{6 g}$ confirmed that carrying out the reaction on aryl-substituted BCB boronate complexes affords the same major diastereoisomer than their alkyl-substituted analogues. The most remarkable evidence is that a NOE correlation between H-3 and the pinacol H-2" was observed in the major diastereoisomer, while absence of this NOE correlation was observed in the minor diastereoisomer.

\section{Mechanistic Considerations}

\subsection{TEMPO Trapping Experiment}

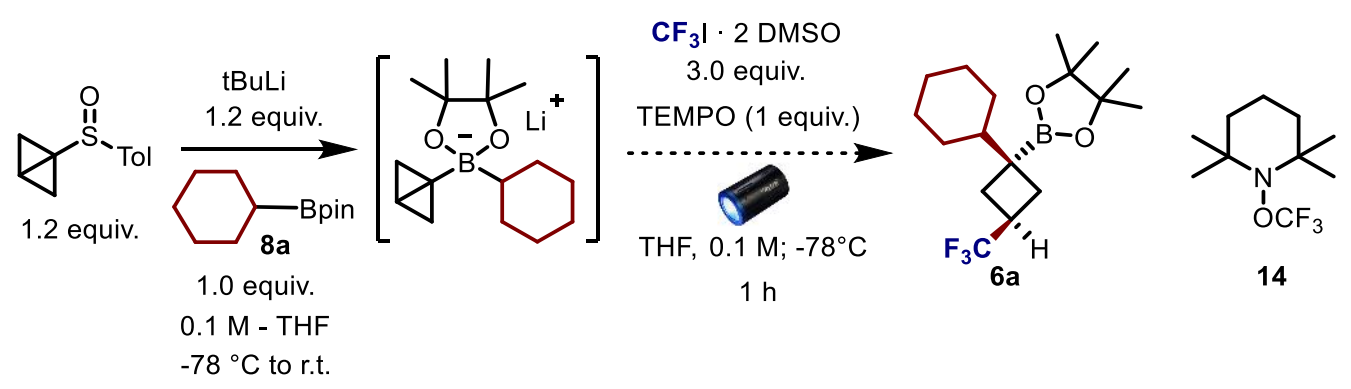

To a $-78{ }^{\circ} \mathrm{C}$ stirred solution of bicyclobutyl sulfoxide 7 (46.0 $\mathrm{mg}, 0.24 \mathrm{mmol}, 1.20$ equiv.) and boronic ester $8 \mathbf{a}\left(0.2 \mathrm{mmol}, 1.0\right.$ equiv.) in THF $(2.0 \mathrm{~mL})$ under $\mathrm{N}_{2}$ atmosphere was added dropwise a pentane solution of tert-butyllithium ( $0.24 \mathrm{mmol}, 1.20$ equiv.). The solution was stirred for 5 minutes at $-78{ }^{\circ} \mathrm{C}$, and left to warm to r.t. under vigorous stirring over 15 minutes, after which it was cooled back to $-78{ }^{\circ} \mathrm{C}$ in a mirror-wall dewar using a cryostat. $31.2 \mathrm{mg}(0.2$ mmol, 1 equiv.) of 2,2,6,6-tetramethyl-1-piperidinyloxy free radical (TEMPO) was added to the cold solution, followed by trifluoromethyl iodide DMSO complex $(211 \mathrm{mg}, 138 \mu \mathrm{L}, 0.60$ mmol, 3.0 equiv.) under a strong $\mathrm{N}_{2}$ flow and under irradiation. The vessel was sealed and the mixture was stirred vigorously for $1 \mathrm{~h}$ or $24 \mathrm{~h}$ at $-78^{\circ} \mathrm{C}$.

After the stated time, benzotrifluoride $(24.5 \mu \mathrm{L}, 0.2 \mathrm{mmol}, 1$ equiv.) was added and an aliquote of the reaction was analysed by ${ }^{19} \mathrm{~F}-\mathrm{NMR}$. Although no product was observed in the crude reaction mixture, compound $\mathbf{1 4}^{\mathbf{1 0}}$ was formed in $8 \%$ yield. 


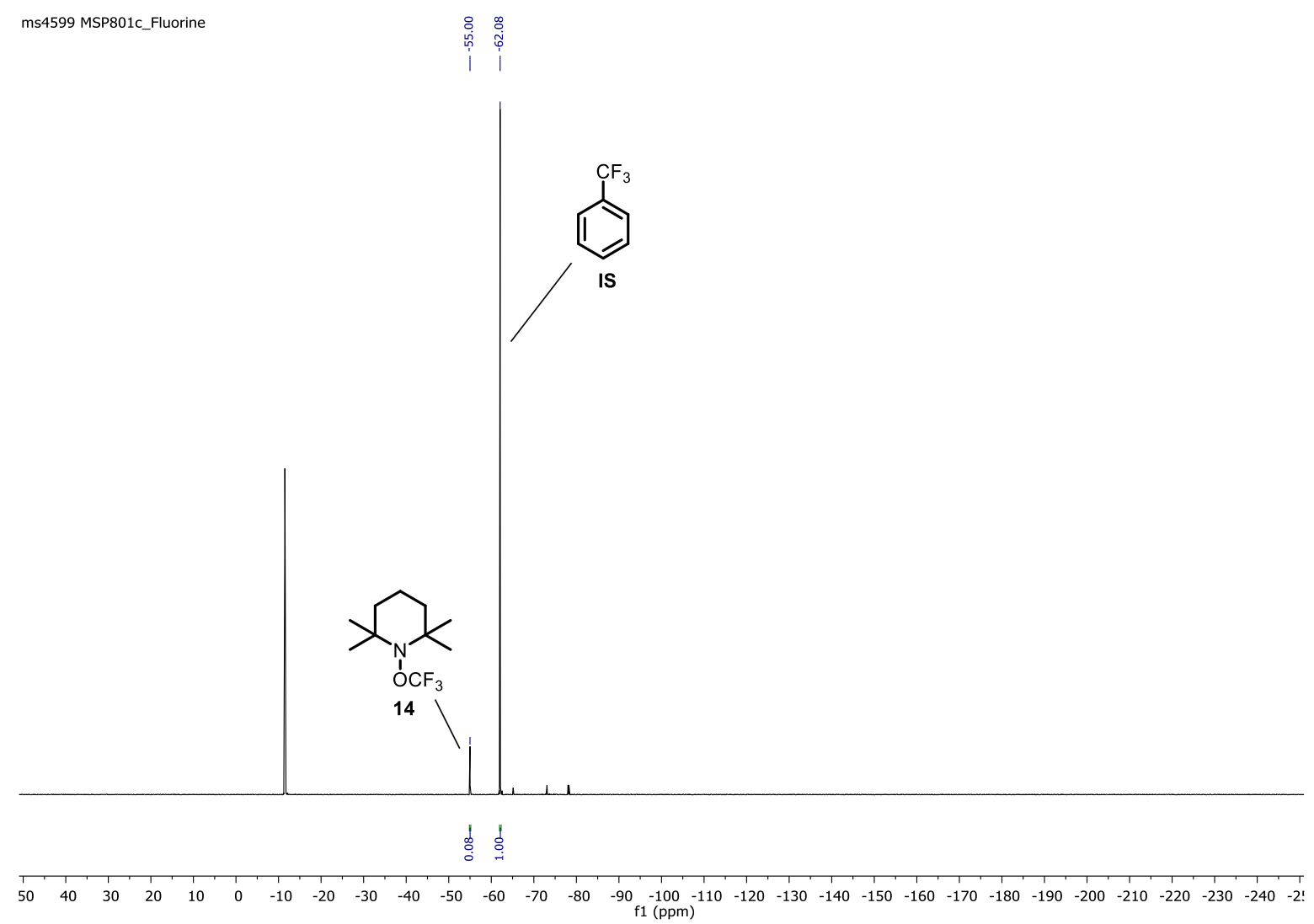

Figure S7. ${ }^{19}$ F-NMR analysis of the reaction crude. Benzotrifluoride (IS) was used as internal standard and the spectrum of compound $\mathbf{1 4}$ was compared to the literature. ${ }^{10}$

\subsection{Aryl-Substituted Substrates}

Compared to their alkyl-substituted analogues, aryl-substituted bicyclobutyl boronate complexes show significantly different behavior. The reactions of these substrates are significantly slower and occur with moderate diastereoselectivity (compare entries 6e-g with 6a-d in the main text). In order to achieve synthetically useful results, for these substrates the reaction time must to be prolonged from 1 to $24 \mathrm{~h}$ and the solvent exchanged to proprionitrile to achieve better stereoselectivity.

Based on the well-known tendency of aromatic groups to undergo 1,2-shift when positioned $\alpha$ to a radical center (neophyl rearrangement), ${ }^{11}$ we believe that an alternative mechanism is likely to be operative with these type of substrates (Scheme S1). 


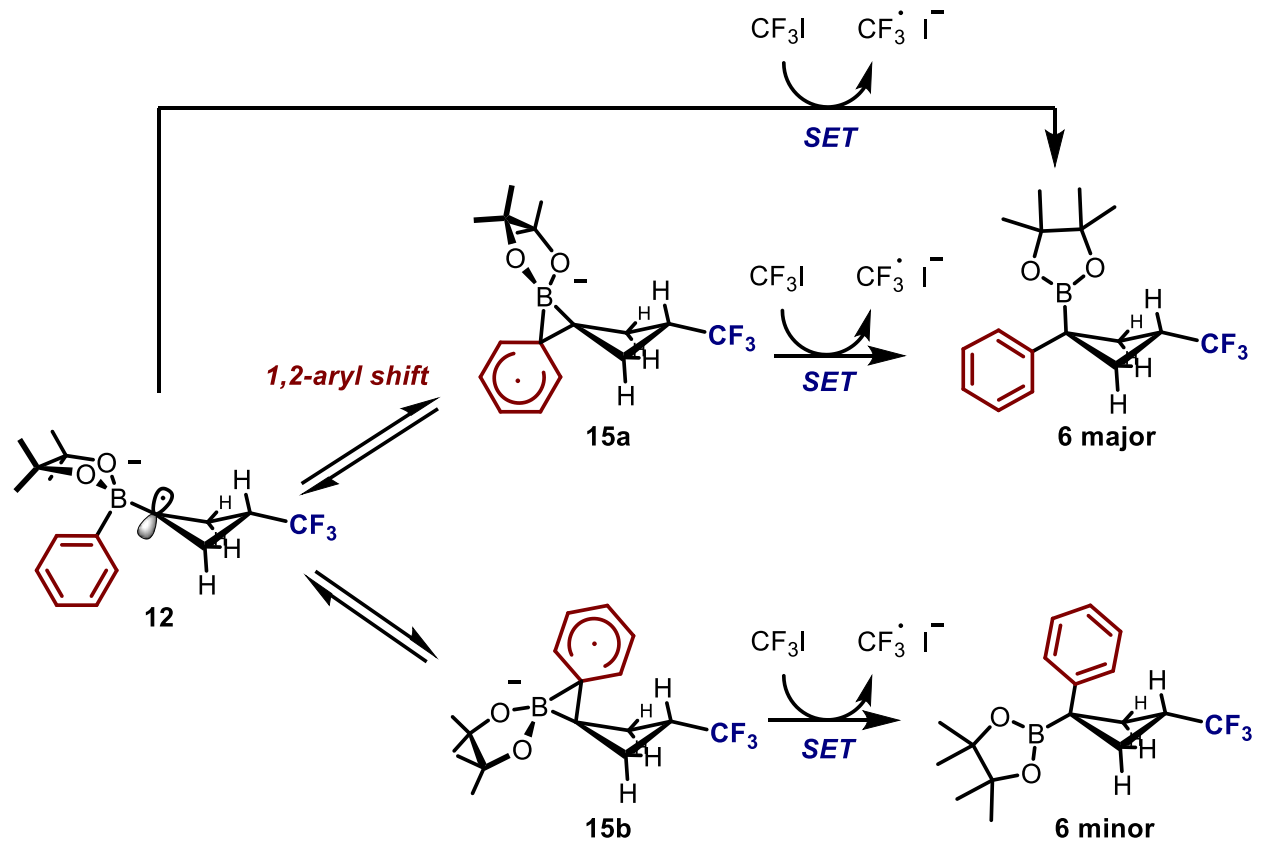

Scheme S1. Mechanistic proposal for aromatic boronate complexes.

For aryl-substituted substrates the radical anion intermediate $\mathbf{1 2}$ is expected to undergo fast rearrangement leading to radical anion intermediates $\mathbf{1 5 a - b}$, which could undergo SET enabling a radical chain mechanism as reported in Scheme 6 (main text). The selectivity of the reaction will be determined by the equilibrium ratio of the radical anion intermediates $\mathbf{1 2} / \mathbf{1 5} \mathbf{a}$ b and their relative rates of SET. The different mechanism operating with aryl substrates compared to alkyl substrates accounts for the different reactivity and selectivity observed.

\section{References}

(1) Filippini, G.; Silvi, M.; Melchiorre, P. Angew. Chem. Int. Ed. 2017, 56, 4447.

(2) D. Noh, H. Chea, J. Ju, J. Yun Angew. Chem. Int. Ed. 2009, 48, 6062.

(3) Aichhorn, S.; Bigler, R.; Myers, E. L.; Aggarwal, V. K. J. Am. Chem. Soc. 2017, 139, 9519.

(4) Fawcett, A.; Biberger, T.; Aggarwal, V. K. Nat. Chem. 2019, 11, 117.

(5) Bonet, A.; Odachowski, M.; Leonori, D.; Essafi, S.; Aggarwal, V. K. Nat. Chem. 2014, 6, 584.

(6) Ito, H.; Kubota, K. Org. Lett. 2012, 14, 890-893.

(7) A. S. Batsanov, C. Grosjean, T. Schutz, A. Whiting J. Org. Chem. 2007, 72, 6276.

(8) Mohamed, N.; Bhatt, U.; Just, G. Tetrahedron Lett. 1998, 39, 8213.

(9) Balieu, S.; Hallett, G. E.; Burns, M.; Bootwicha, T.; Studley, J.; Aggarwal, V. K. J. Am. Chem. Soc. 2015, 137, 4398.

(10) Ji, G.; Wang, X.; Zhang, S.; Xu, Y.; Ye, Y.; Li, M.; Zhang, Y.; Wang, J. Chem. Commun. 2014, 50, 4361.

(11) a) Seubold, F. H. J. Am. Chem. Soc. 1953, 75, 2532. b) Chen, Z.-M.; Zhang, X.-M.; Tu, Y.-Q. Chem. Soc. Rev. 2015, 44, 5220. 


\section{HPLC Chromatograms}

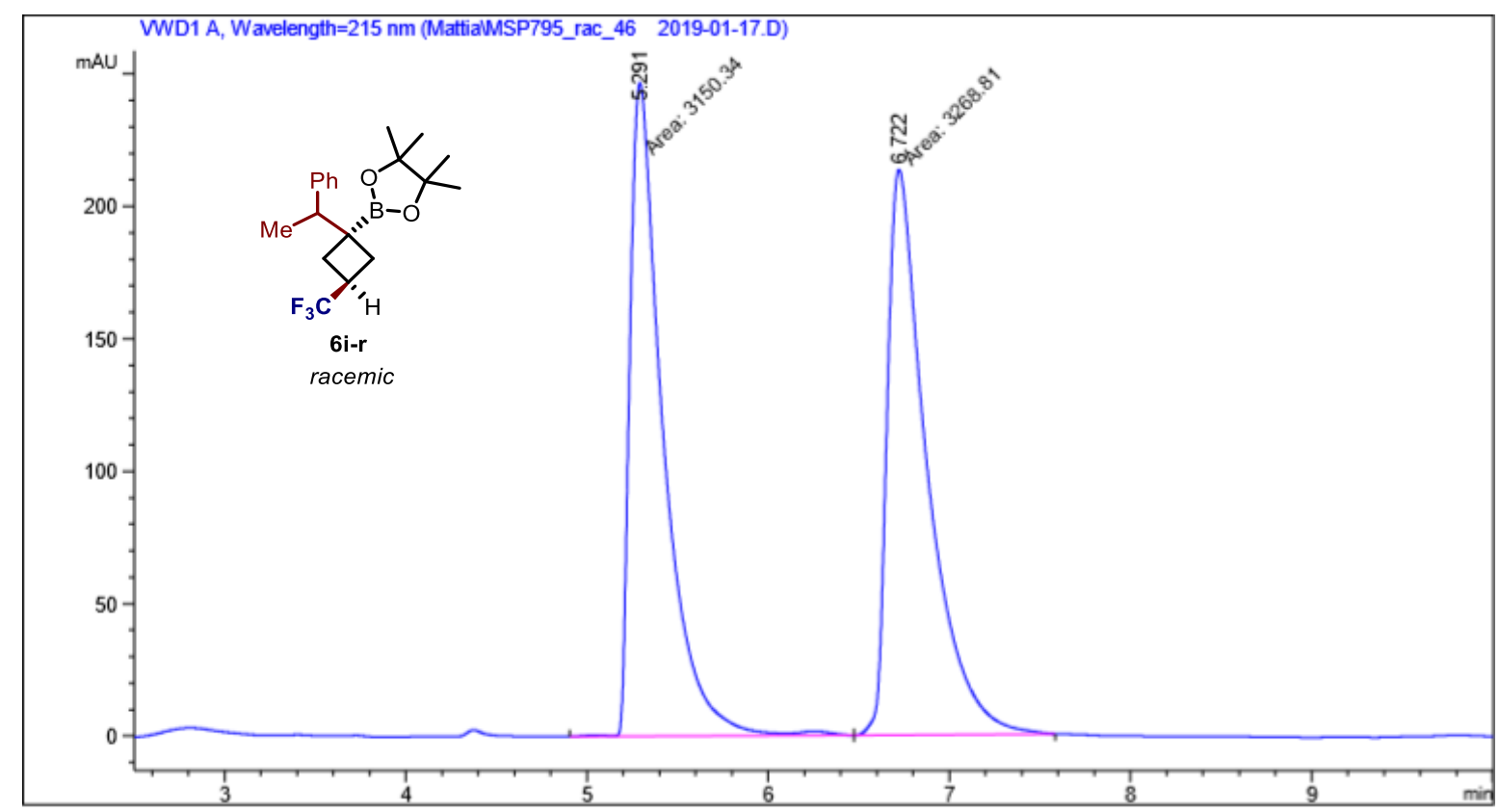

Signal 1: VWD1 $\mathrm{A}$, Wavelength $=215 \mathrm{~nm}$
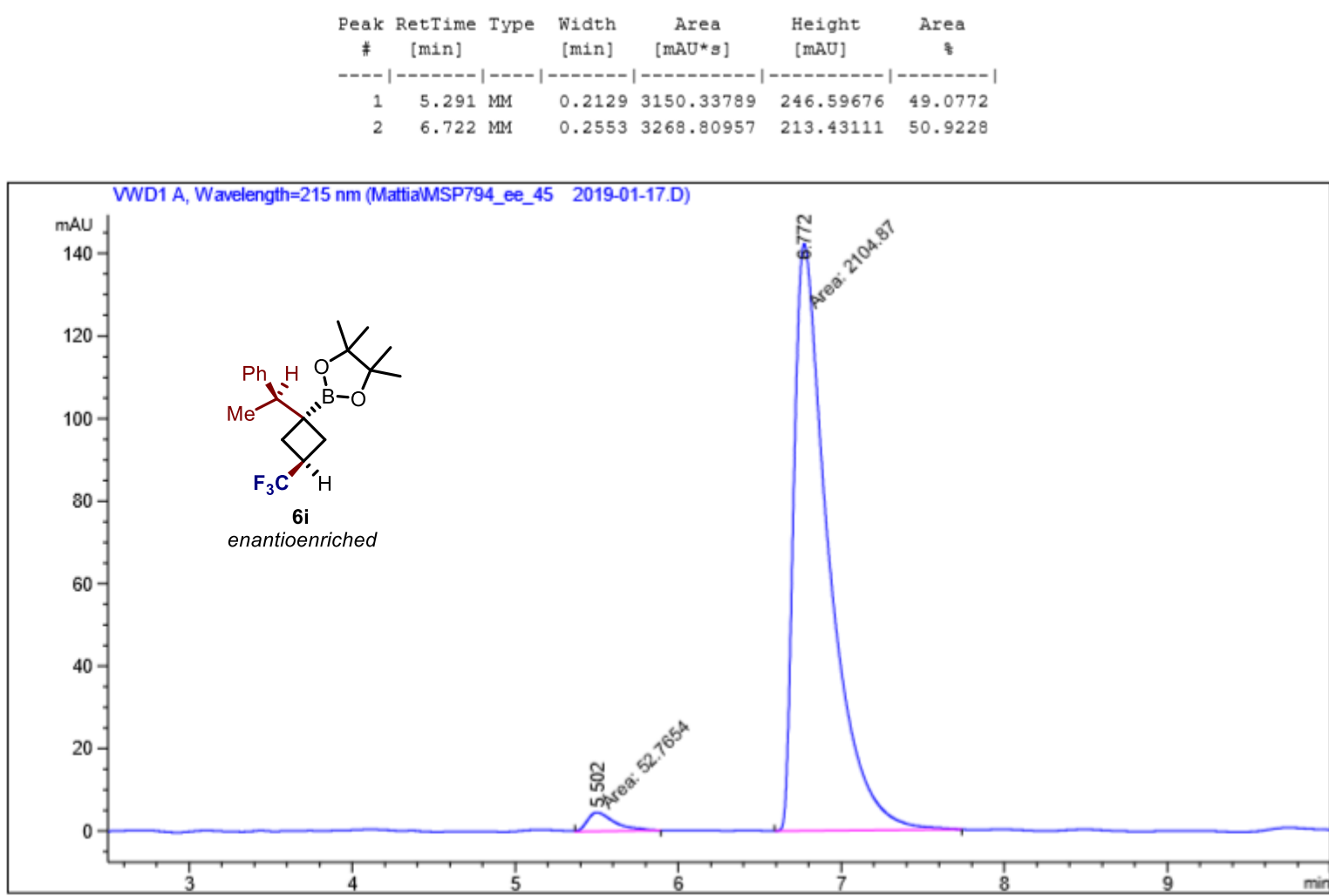

Signal 1: VWD1 $\mathrm{A}$, Wavelength=215 nm

\begin{tabular}{|c|c|c|c|c|c|c|}
\hline $\begin{array}{c}\text { Peak } \\
\#\end{array}$ & $\begin{array}{c}\text { RetTime } \\
\text { [min] }\end{array}$ & Type & $\begin{array}{l}\text { Width } \\
\text { [min] }\end{array}$ & $\begin{array}{c}\text { Area } \\
{[\mathrm{mAU} * \mathrm{~s}]}\end{array}$ & $\begin{array}{l}\text { Height } \\
\text { [mAU] }\end{array}$ & $\underset{*}{\text { Area }}$ \\
\hline & -- & & -- & - - & ------1 & 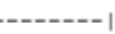 \\
\hline 1 & 5.502 & MM & 0.1947 & 52.76536 & 4.51740 & 2.4455 \\
\hline 2 & 6.772 & MM & 0.2467 & 2104.87085 & 142.18610 & 97.5545 \\
\hline
\end{tabular}




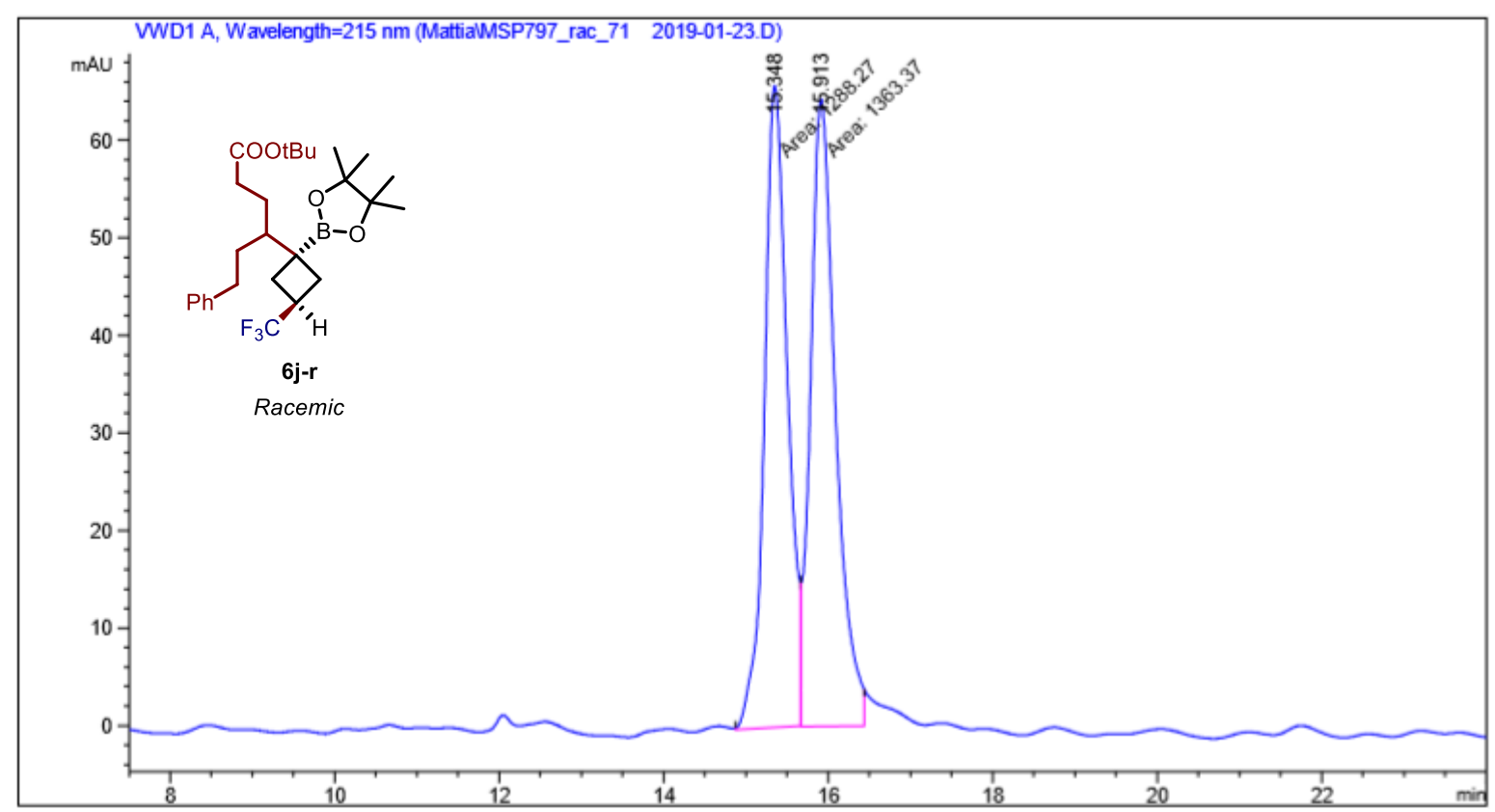

Signal 1: VWD1 A, Wavelength=215 nm

\begin{tabular}{|c|c|c|c|c|c|c|}
\hline $\begin{array}{c}\text { Peak } \\
\#\end{array}$ & $\begin{array}{c}\text { RetTime } \\
\text { [min] }\end{array}$ & Type & $\begin{array}{c}\text { Width } \\
\text { [min] }\end{array}$ & $\begin{array}{c}\text { Area } \\
{\left[\mathrm{mAU} \mathbf{U}^{*} \mathrm{~s}\right]}\end{array}$ & $\begin{array}{l}\text { Height } \\
\text { [mAU] }\end{array}$ & $\begin{array}{c}\text { Area } \\
\text { \& }\end{array}$ \\
\hline & & & - & $------m$ & --n-n-- & 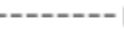 \\
\hline 1 & 15.348 & $\mathrm{MM}$ & 0.3265 & 1288.27478 & 65.75306 & 48.5839 \\
\hline 2 & 15.913 & MM & 0.3535 & 1363.37231 & 64.27234 & 51.4161 \\
\hline
\end{tabular}

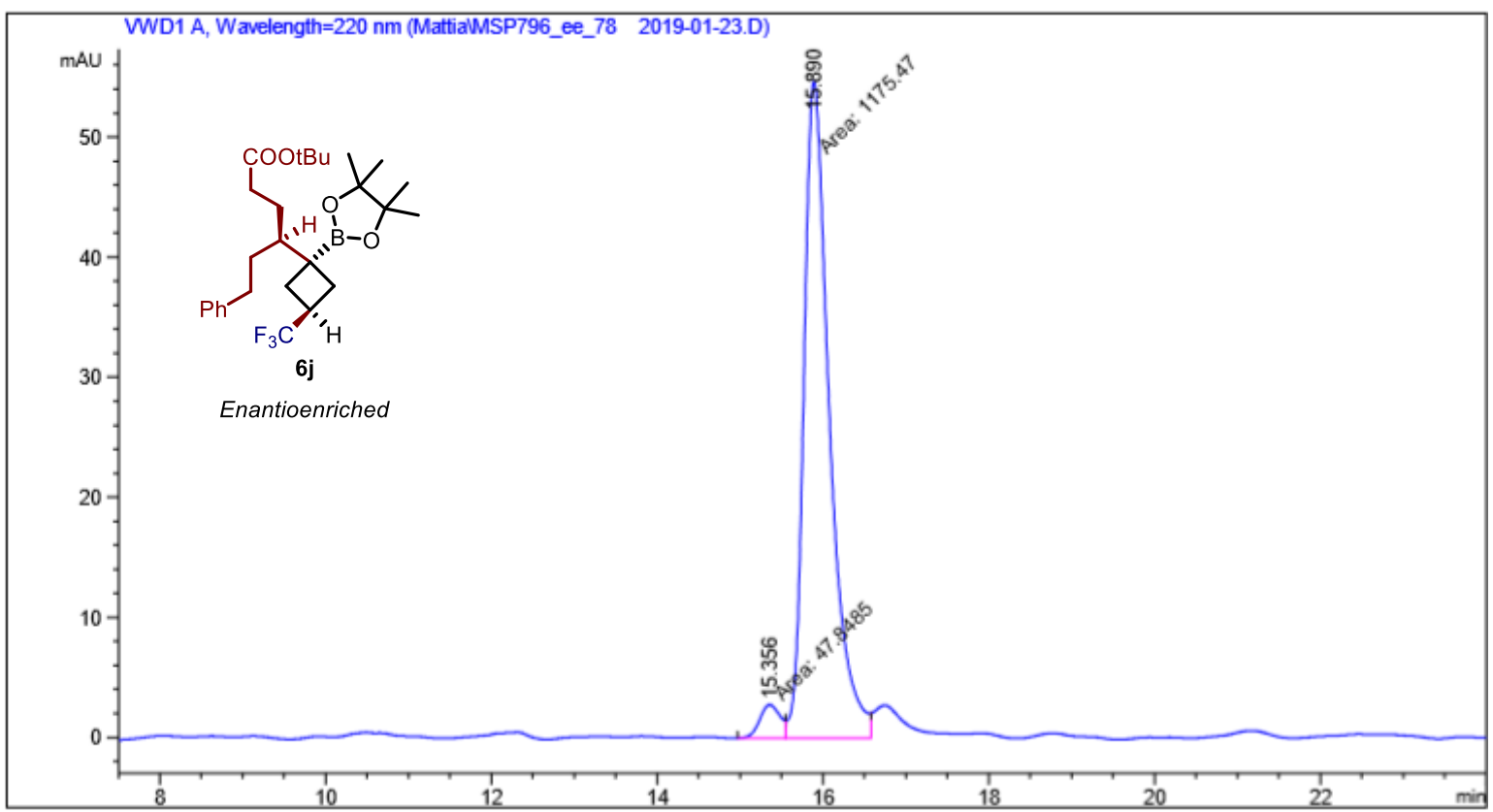

Signal 1: VWD1 A, Wavelength=220 nm

\begin{tabular}{cccccc}
$\begin{array}{c}\text { Peak RetTime Type } \\
\text { \# } \\
\text { [min] }\end{array}$ & $\begin{array}{c}\text { Width } \\
\text { [min] }\end{array}$ & $\begin{array}{c}\text { Area } \\
\text { [mAU*s] }\end{array}$ & $\begin{array}{c}\text { Height } \\
\text { [mAU] }\end{array}$ & $\begin{array}{c}\text { Area } \\
\text { * }\end{array}$ \\
\hline-1 & $15.356 \mathrm{MM}$ & 0.2877 & 47.84850 & 2.77208 & 3.9114 \\
2 & $15.890 \mathrm{MM}$ & 0.3584 & 1175.46936 & 54.66457 & 96.0886
\end{tabular}




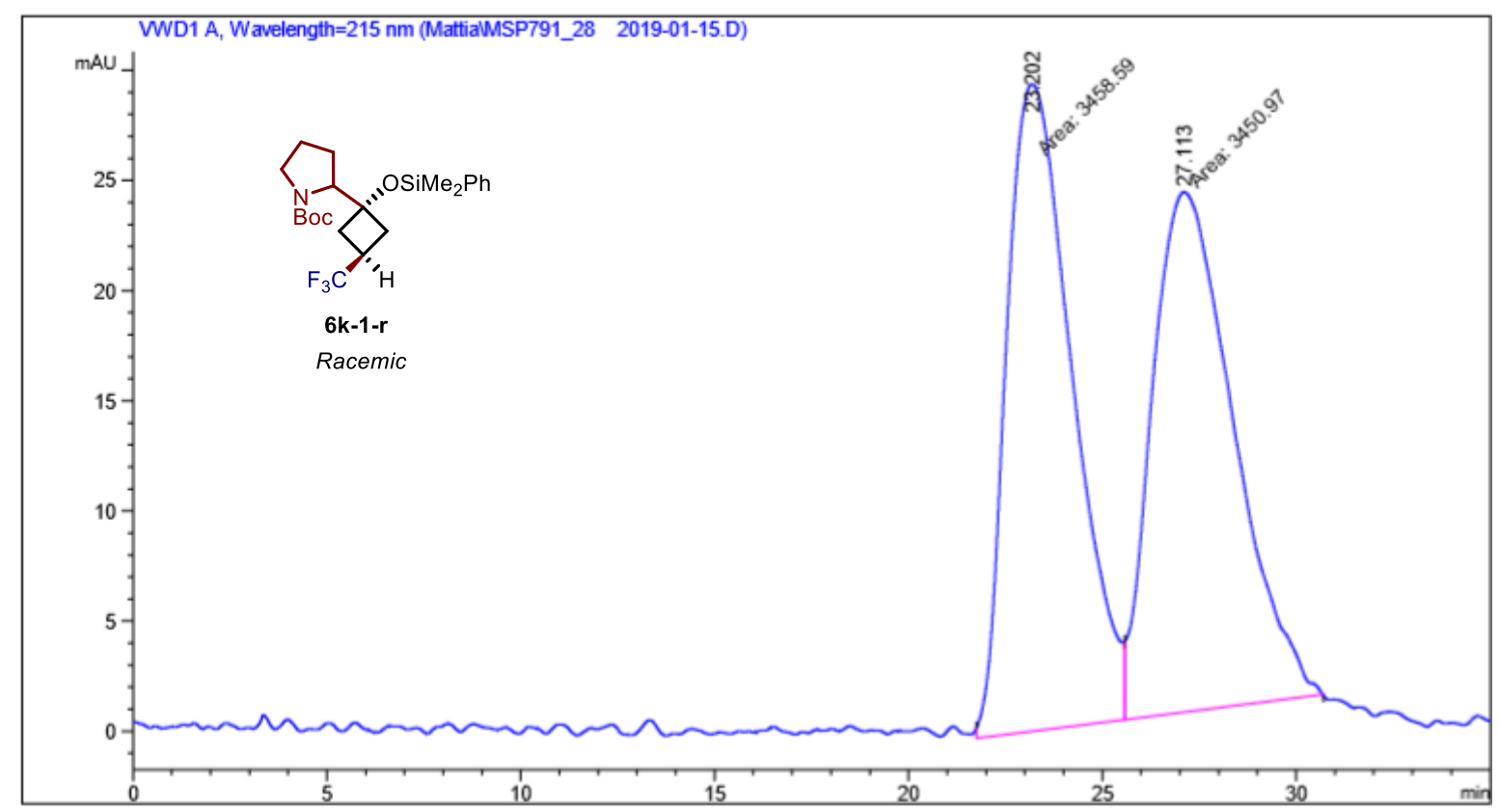

Signal 1: VWD1 A, Wavelength=215 nm

\begin{tabular}{|c|c|c|c|c|c|c|}
\hline $\begin{array}{c}\text { Peak } \\
\#\end{array}$ & $\begin{array}{c}\text { RetTime } \\
\text { [min] }\end{array}$ & Type & $\begin{array}{l}\text { Width } \\
\text { [min] }\end{array}$ & $\begin{array}{c}\text { Area } \\
{\left[\mathrm{mAU}^{*} 3\right]}\end{array}$ & $\begin{array}{l}\text { Height } \\
\text { [mAU] }\end{array}$ & Area \\
\hline & & & 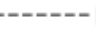 & 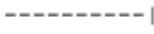 & -2.0 & ---1 \\
\hline 1 & 23.202 & MM & 1.9630 & 3458.59033 & 29.36535 & 50.0552 \\
\hline 2 & 27.113 & MM & 2.4369 & 3450.96631 & 23.60169 & 49.9448 \\
\hline
\end{tabular}

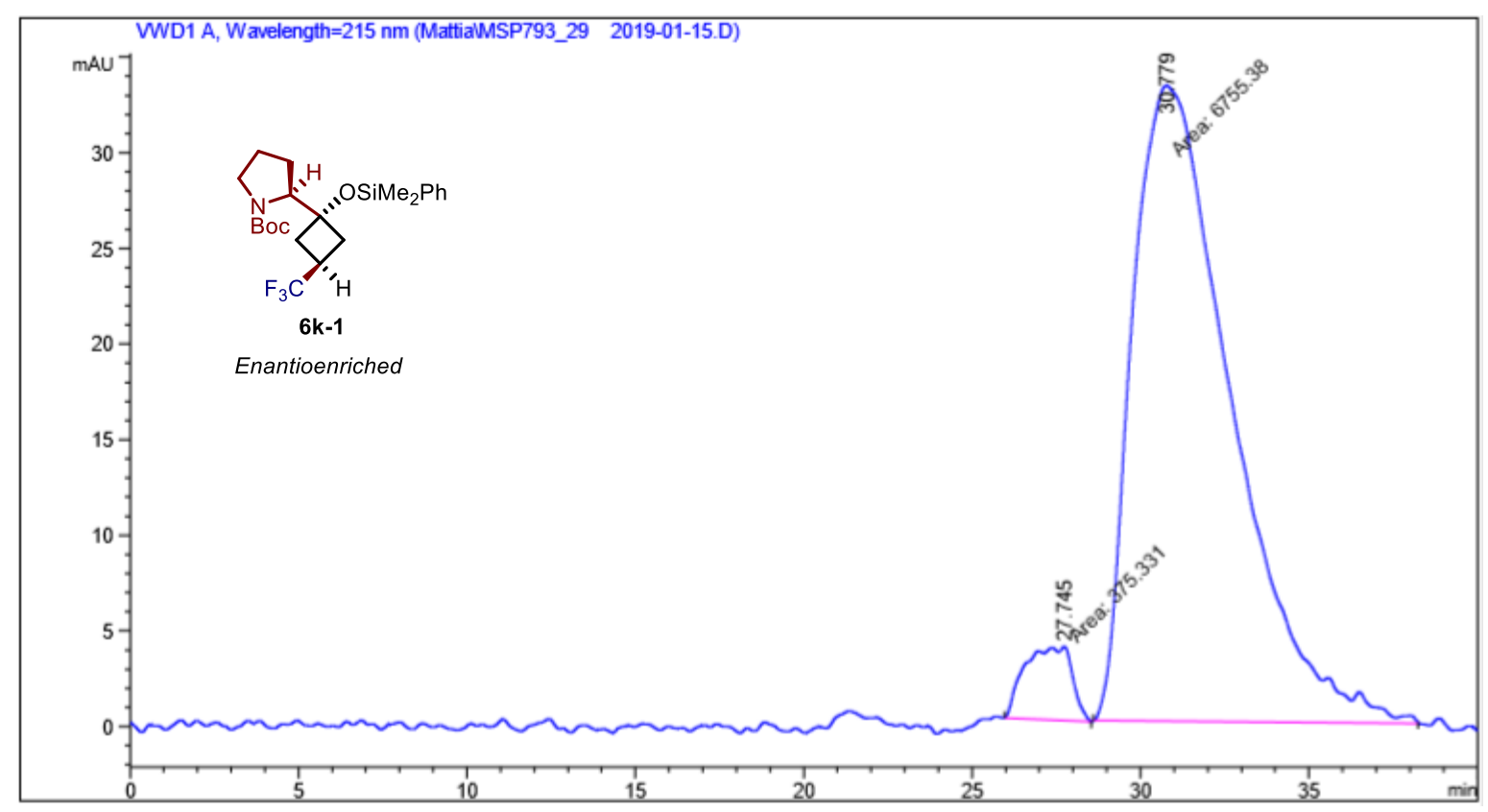

Signal 1: VWD1 A, Wavelength=215 nm

\begin{tabular}{|c|c|c|c|c|c|c|}
\hline $\begin{array}{c}\text { Peak } \\
\#\end{array}$ & $\begin{array}{l}\text { RetTime } \\
\text { [min] }\end{array}$ & Type & $\begin{array}{l}\text { Width } \\
\text { [min] }\end{array}$ & $\begin{array}{c}\text { Area } \\
{\left[\mathrm{mAO}{ }^{*} \mathrm{~s}\right]}\end{array}$ & $\begin{array}{l}\text { Height } \\
\text { [mAU] }\end{array}$ & $\underset{\text { A }}{\text { Area }}$ \\
\hline & & & & & & \\
\hline 1 & 27.745 & MM & 1.6137 & 375.33081 & 3.87660 & 5.2636 \\
\hline 2 & 30.779 & MM & 3.3878 & 6755.38184 & 33.23375 & 94.7364 \\
\hline
\end{tabular}




\section{NMR Spectra}

${ }^{13} \mathrm{C}$ NMR of compound $5 \mathbf{u}\left(500 \mathrm{MHz}, \mathrm{CDCl}_{3}\right)$

ms22520_MSP813WU_PROTON_001<smiles>COC(=O)[C@H](Cc1ccccc1)NC(=O)[C@H](C)NC(=O)CI</smiles>
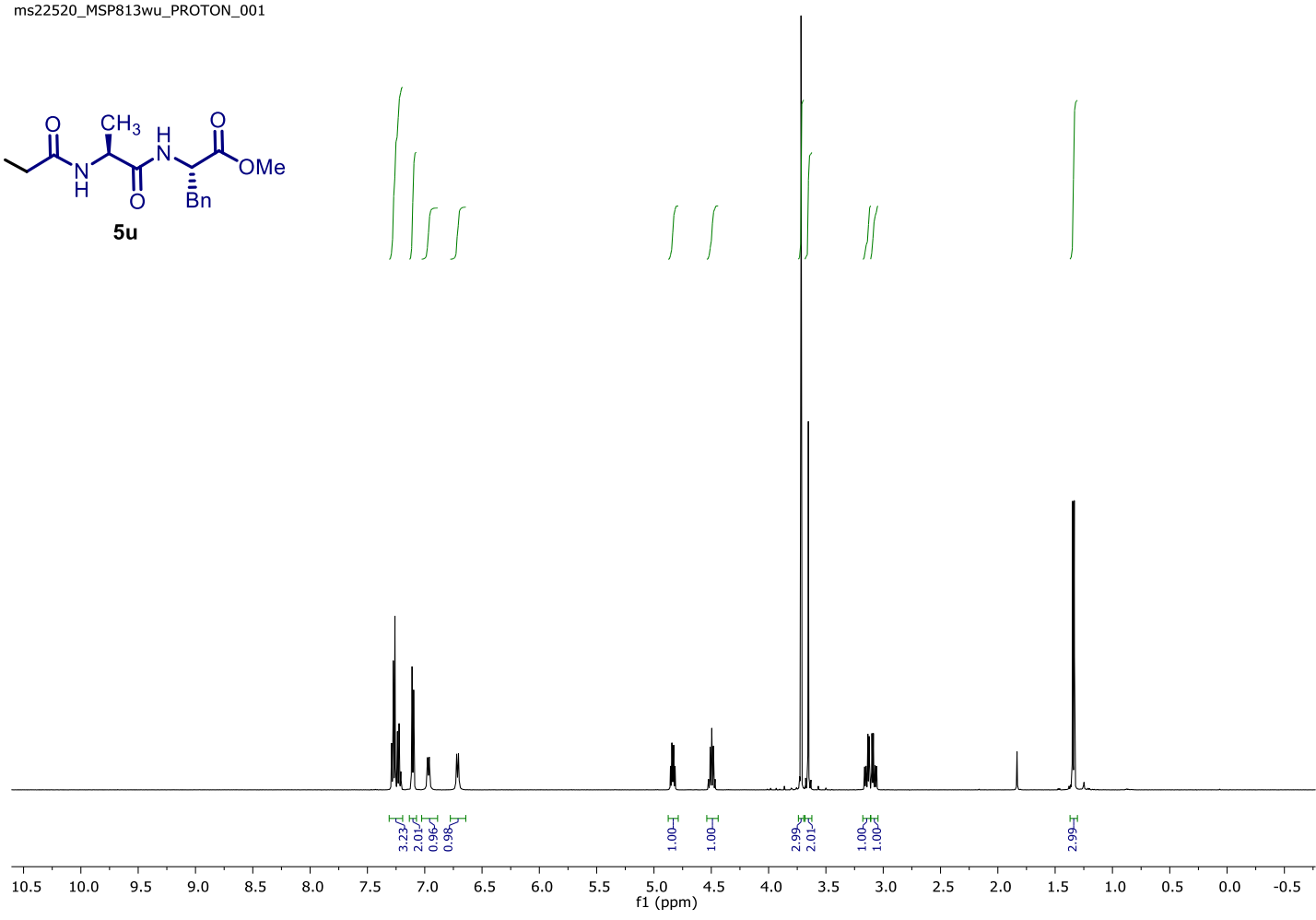

${ }^{13} \mathrm{C}$ NMR of compound $5 \mathbf{u}\left(125 \mathrm{MHz}, \mathrm{CDCl}_{3}\right)$ ms22520_MSP813WU_CARBON_001-

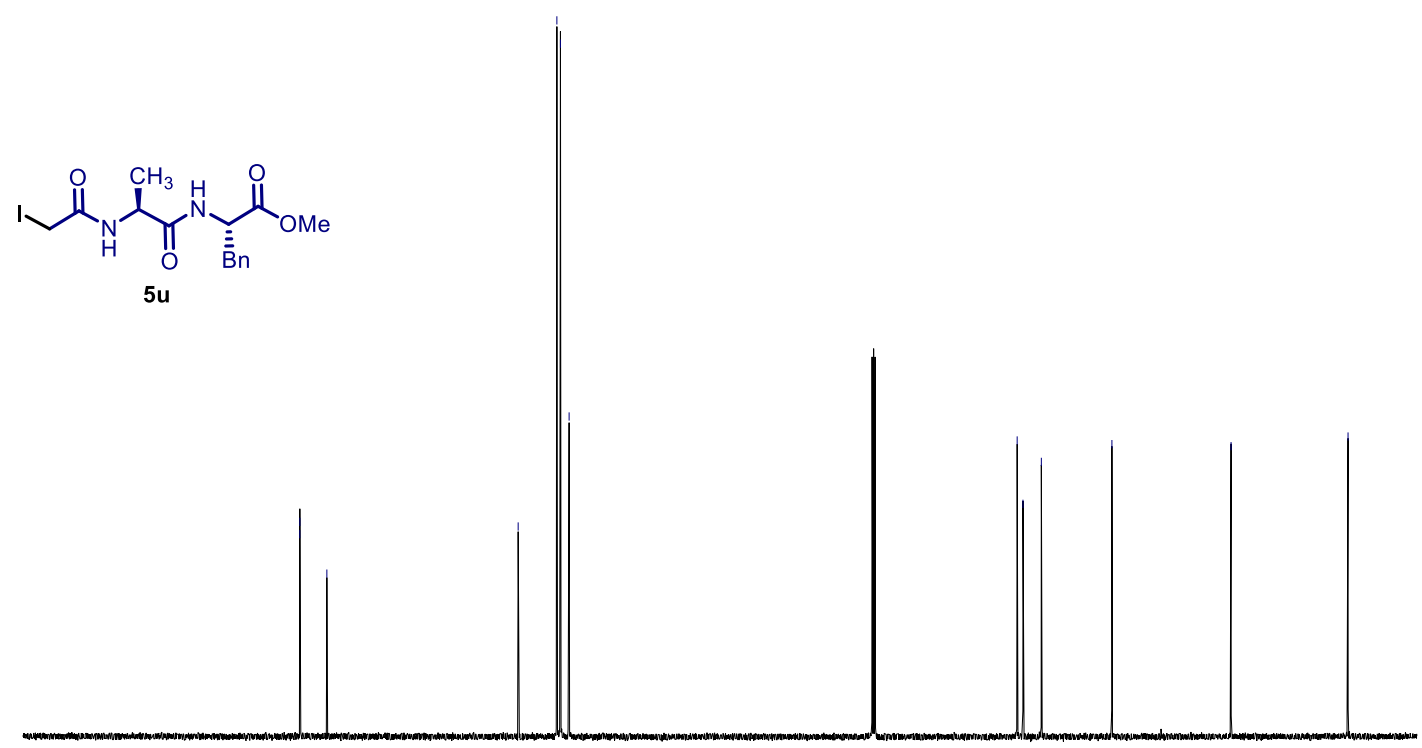

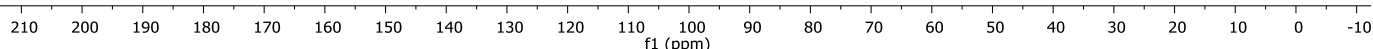


${ }^{1} \mathrm{H}$ NMR of compound $\mathbf{5 v}\left(500 \mathrm{MHz}, \mathrm{CDCl}_{3}\right)$

va/ms6264 MSP818wu
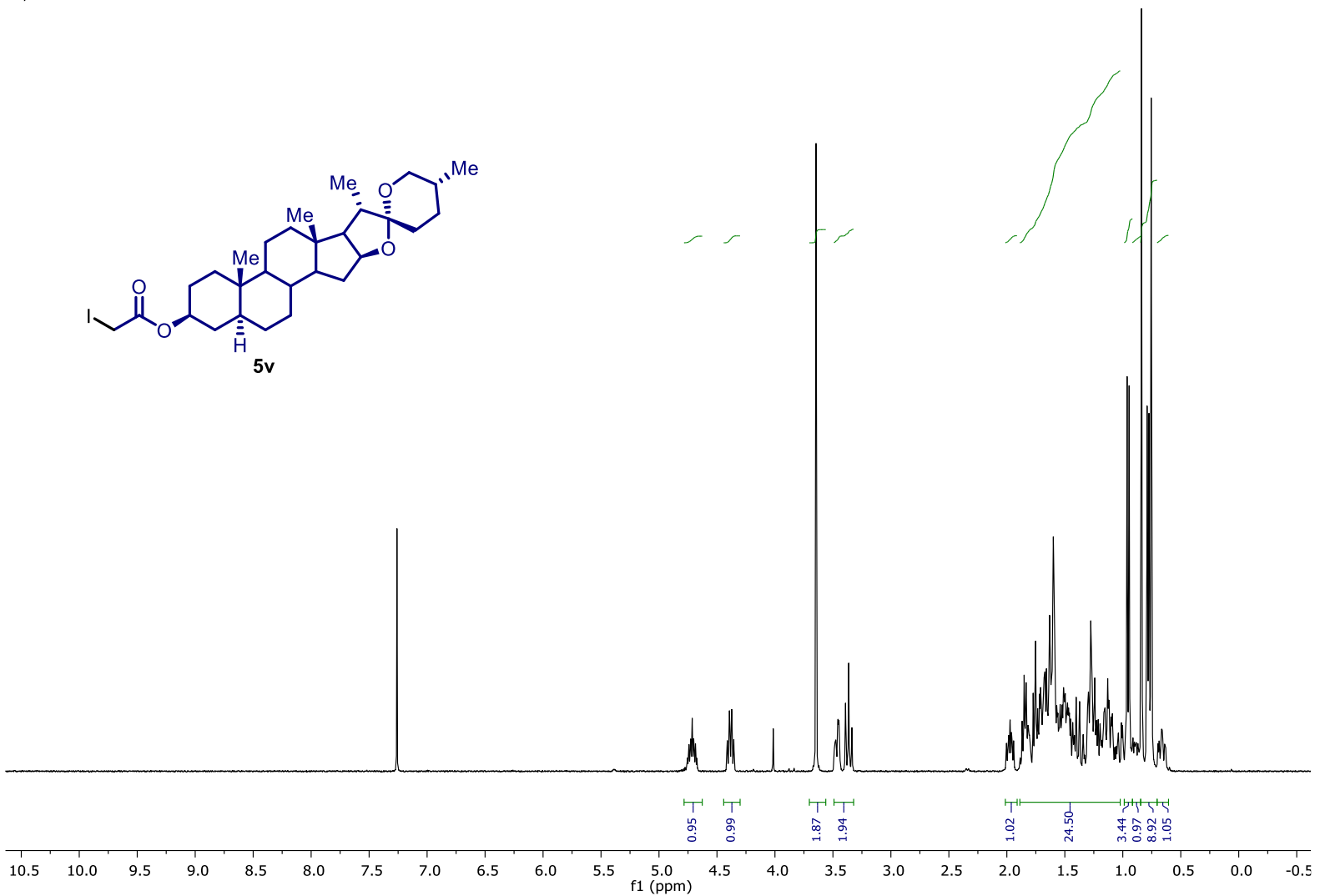

${ }^{13} \mathrm{C}$ NMR of compound $\mathbf{5 v}\left(125 \mathrm{MHz}, \mathrm{CDCl}_{3}\right)$

va/ms6264 MSP818wu

$$
\underset{\substack{n \\ \infty}}{\substack{0 \\ \stackrel{1}{1}}}
$$

|
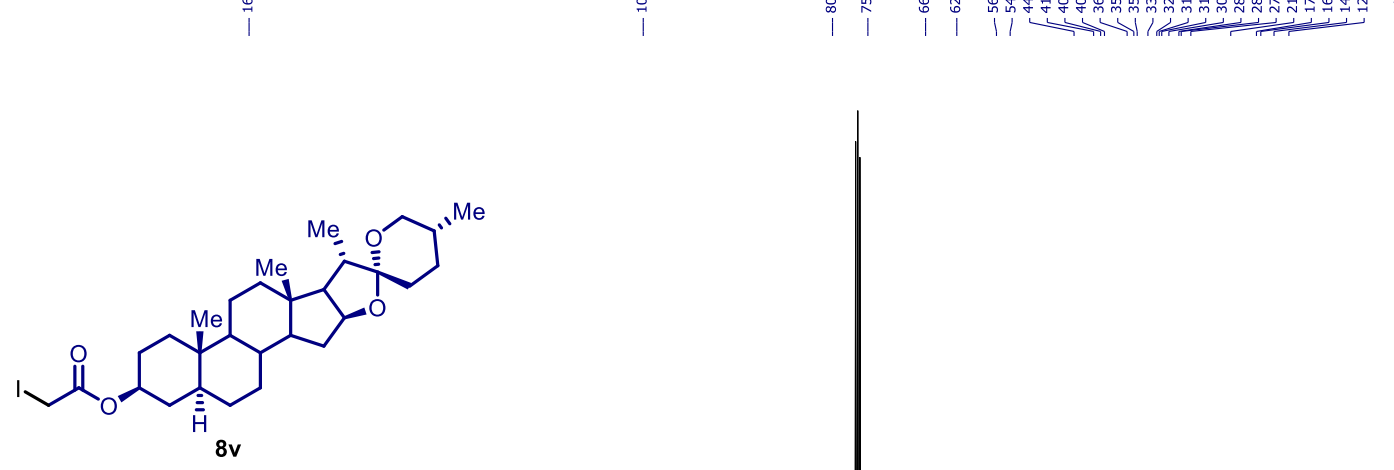

$\begin{array}{lllllllllll}210 & 200 & 190 & 180 & 170 & 160 & 150 & 140 & 130 & 120 & 110 \\ \mathrm{f} 1(\mathrm{ppm})\end{array}$ 
${ }^{1} \mathrm{H}$ NMR of compound $\mathbf{6 a}\left(500 \mathrm{MHz}, \mathrm{CDCl}_{3}\right)$

$\mathrm{va} / \mathrm{ms} 78468$ MSP746P

single_pulse
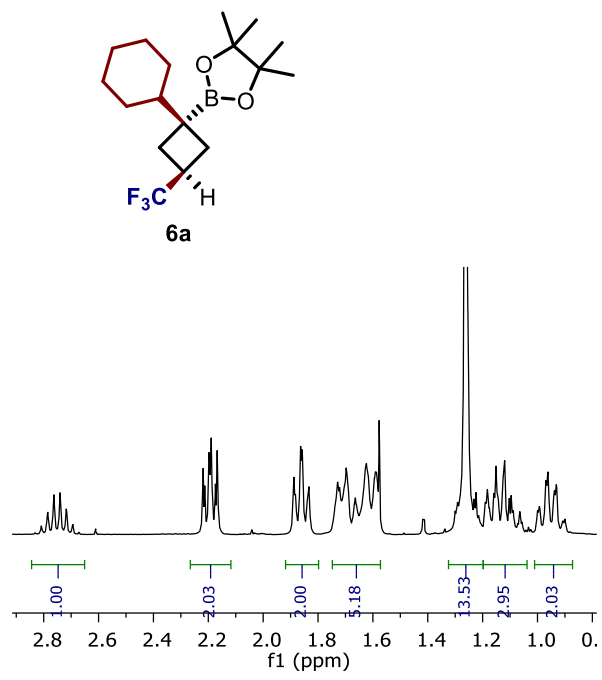

M. M M M d

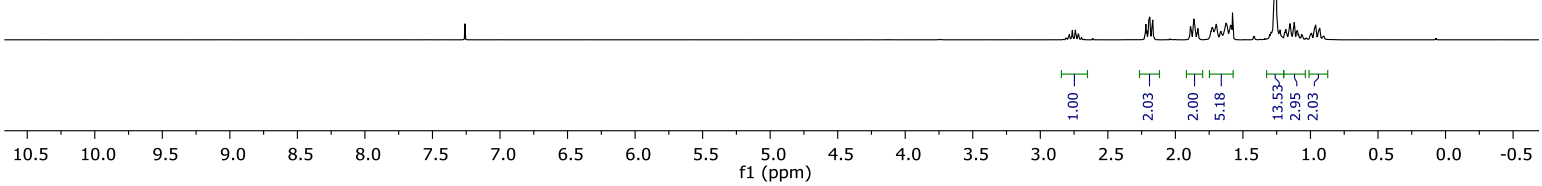

${ }^{13} \mathrm{C}$ NMR of compound $6 \mathbf{6 a}\left(125 \mathrm{MHz}, \mathrm{CDCl}_{3}\right)$

ms26598_MSP715p_CF3_CARBON_001

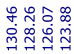

111

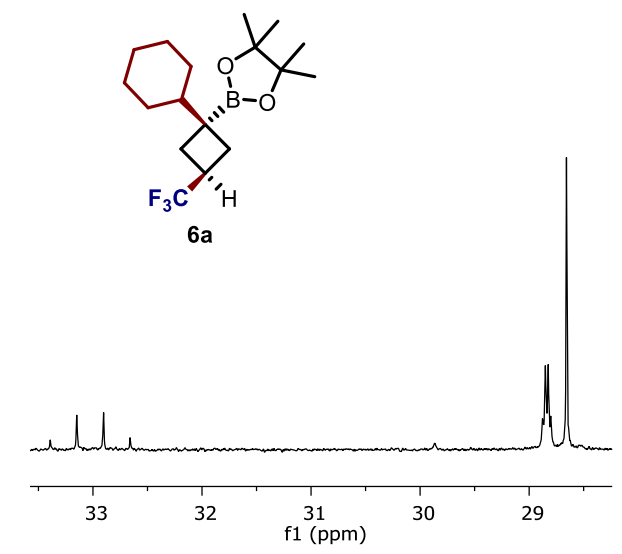

$6 a$

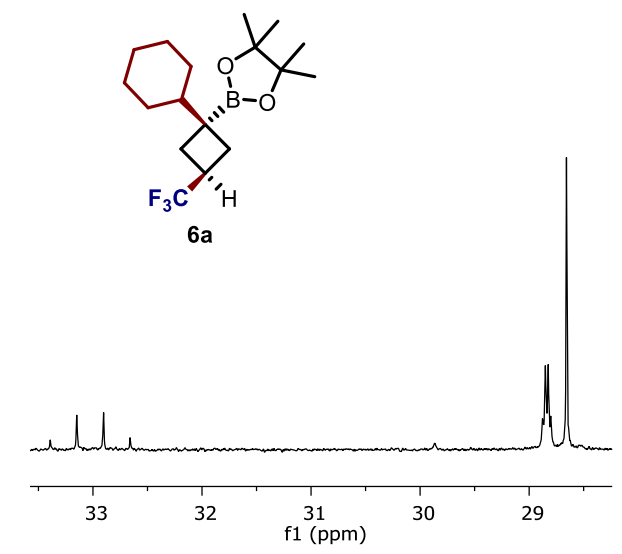

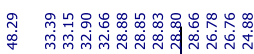
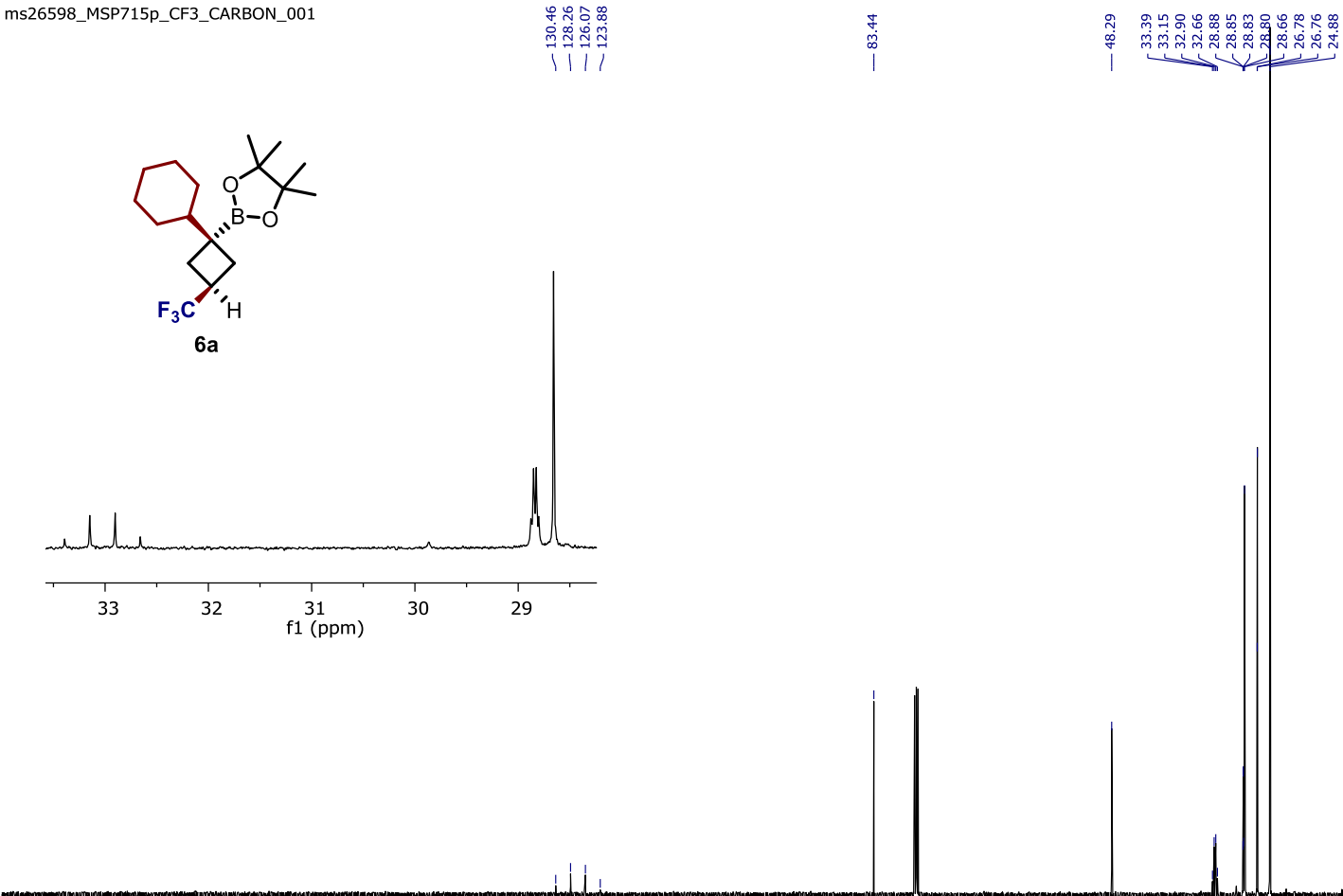

$\begin{array}{llllllllllll}210 & 200 & 190 & 180 & 170 & 160 & 150 & 140 & 130 & 120 & 110 & 100\end{array}$ 
${ }^{19} \mathrm{~F}$ NMR of compound $\mathbf{6 a}\left(\mathrm{CDCl}_{3}, 377 \mathrm{MHz}\right)$

va/ms78468 MSP746p

19F_single_pulse

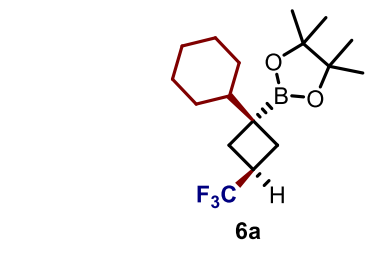

竞表

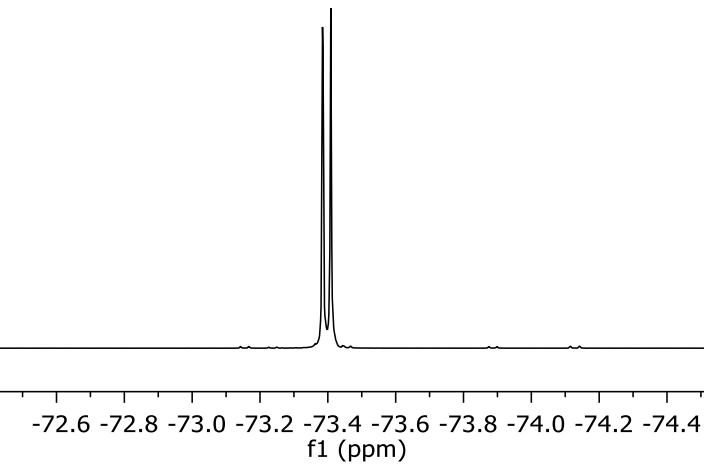

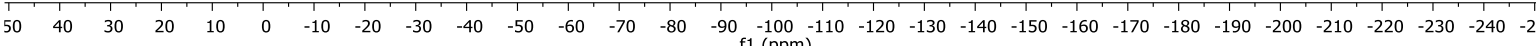


${ }^{1} \mathrm{H}$ NMR of compound $\mathbf{6 b}\left(500 \mathrm{MHz}, \mathrm{CDCl}_{3}\right)$

ms17100_MSP754P_PROTON01
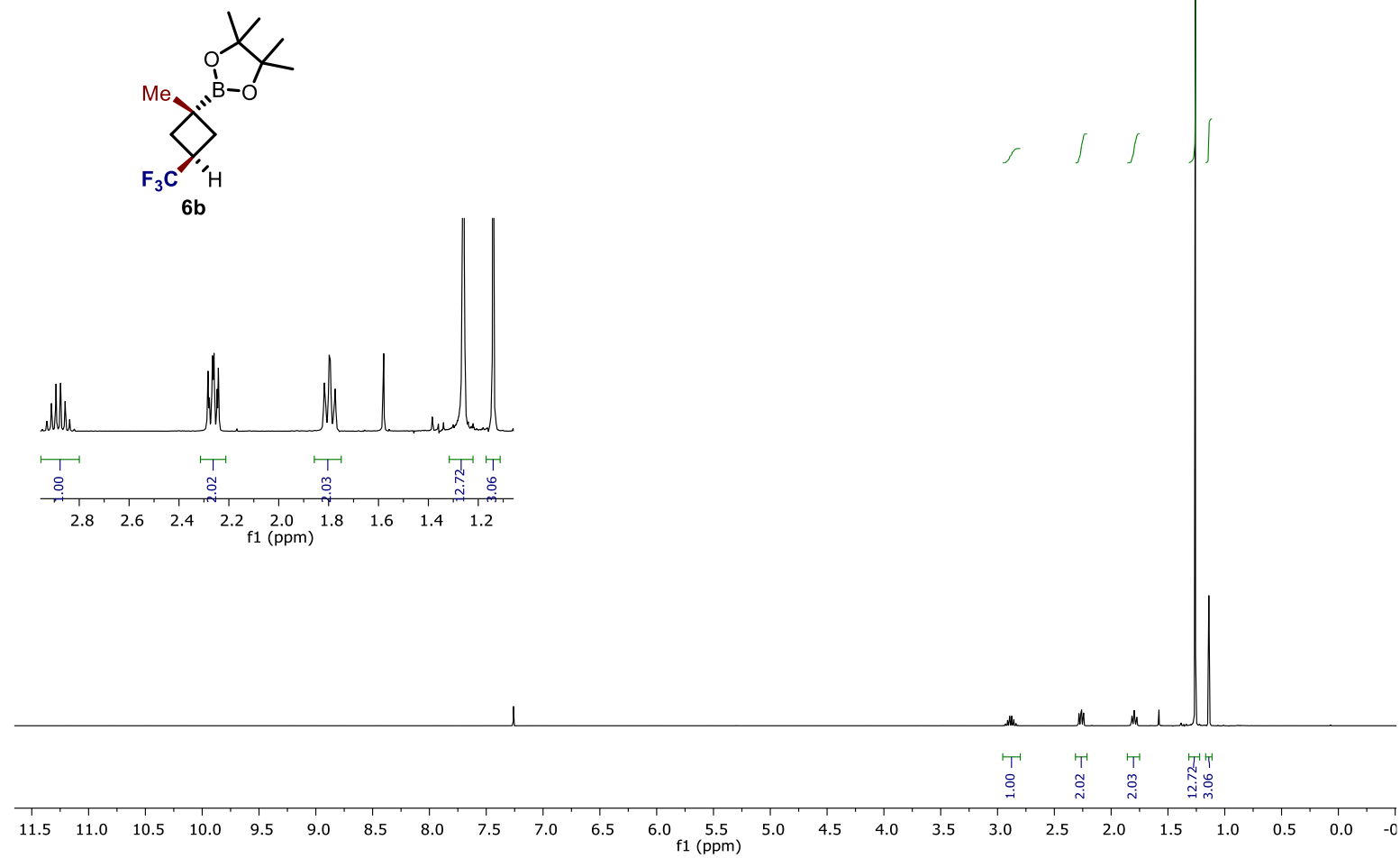

${ }^{13} \mathrm{C}$ NMR of compound $6 \mathbf{b}\left(125 \mathrm{MHz}, \mathrm{CDCl}_{3}\right)$

ms17100_MSP754p_CARBON01

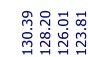

१1

$\stackrel{\substack{\hat{n} \\ \infty}}{\substack{\infty \\ \mid}}$

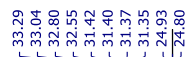
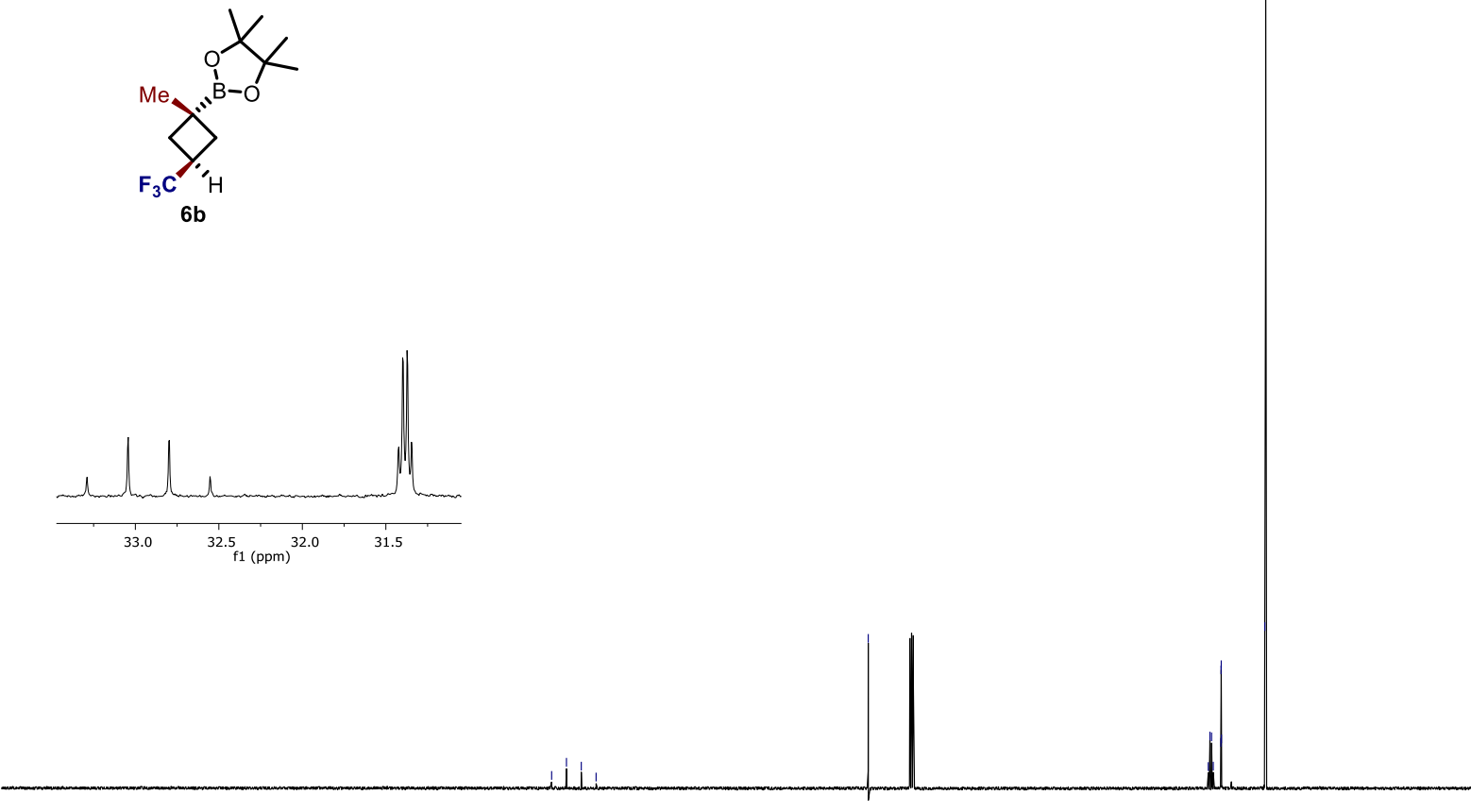

$\begin{array}{lllllllllll}210 & 200 & 190 & 180 & 170 & 160 & 150 & 140 & 130 & 120 & 110 \\ \mathrm{f} 1(\mathrm{ppm}) & 100\end{array}$ 
${ }^{19} \mathrm{~F}$ NMR of compound $\mathbf{6 b}\left(377 \mathrm{MHz}, \mathrm{CDCl}_{3}\right)$

$\mathrm{va} / \mathrm{ms} 7884$ MSP754p 19F_single_pulse

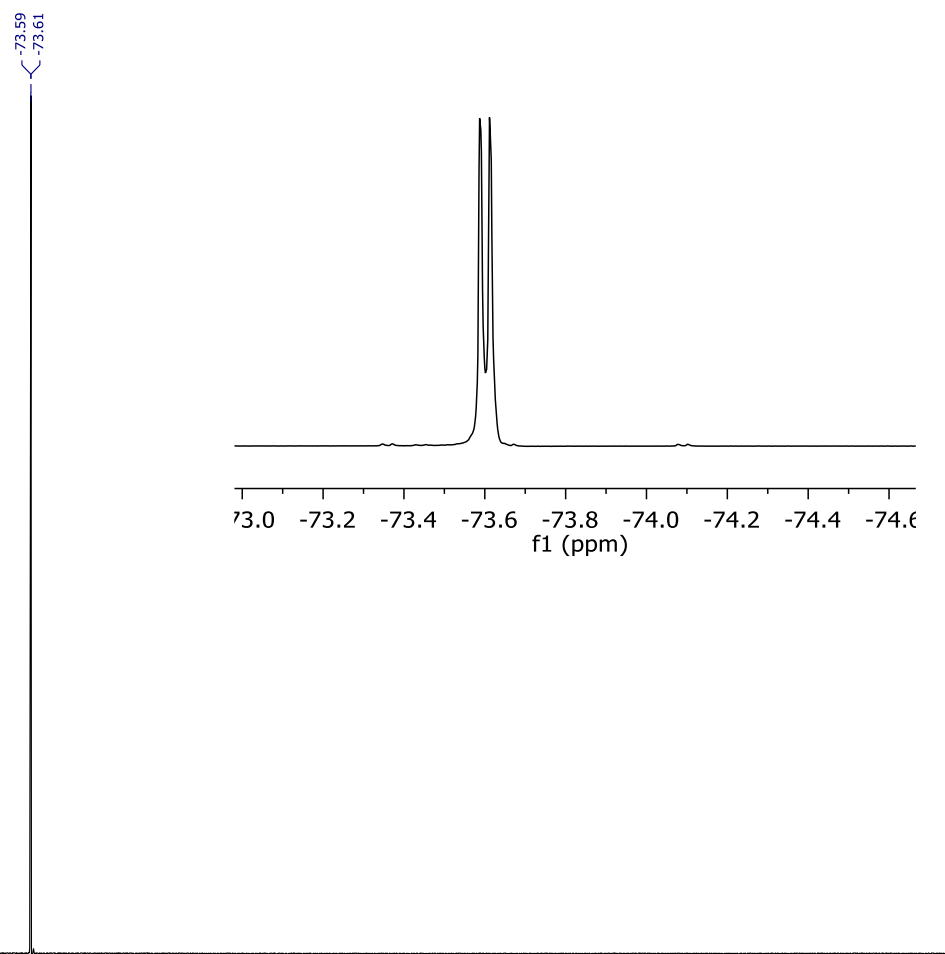

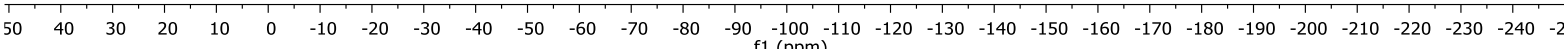


${ }^{1} \mathrm{H}$ NMR of compound $\mathbf{6 c}\left(400 \mathrm{MHz}, \mathrm{CDCl}_{3}\right)$

ms43124_MSP747p_PROTON_01
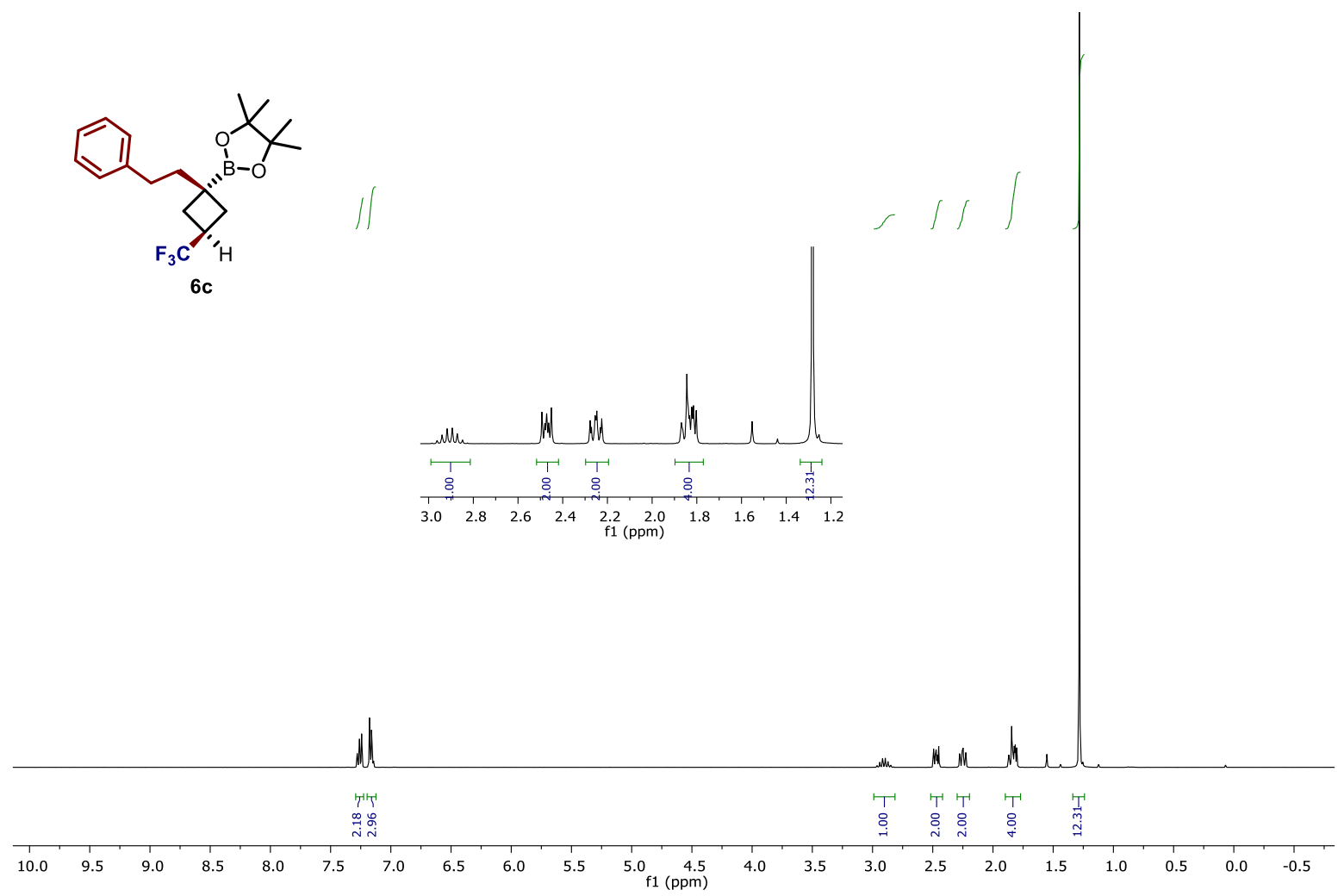

${ }^{13} \mathrm{C}$ NMR of compound $\mathbf{6 c}\left(100 \mathrm{MHz}, \mathrm{CDCl}_{3}\right)$

ms43124_MSP747p_CARBON_01

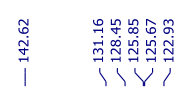

$\underset{\substack{p \\ \infty}}{\substack{n \\ \text { I }}}$

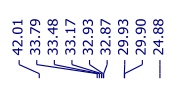
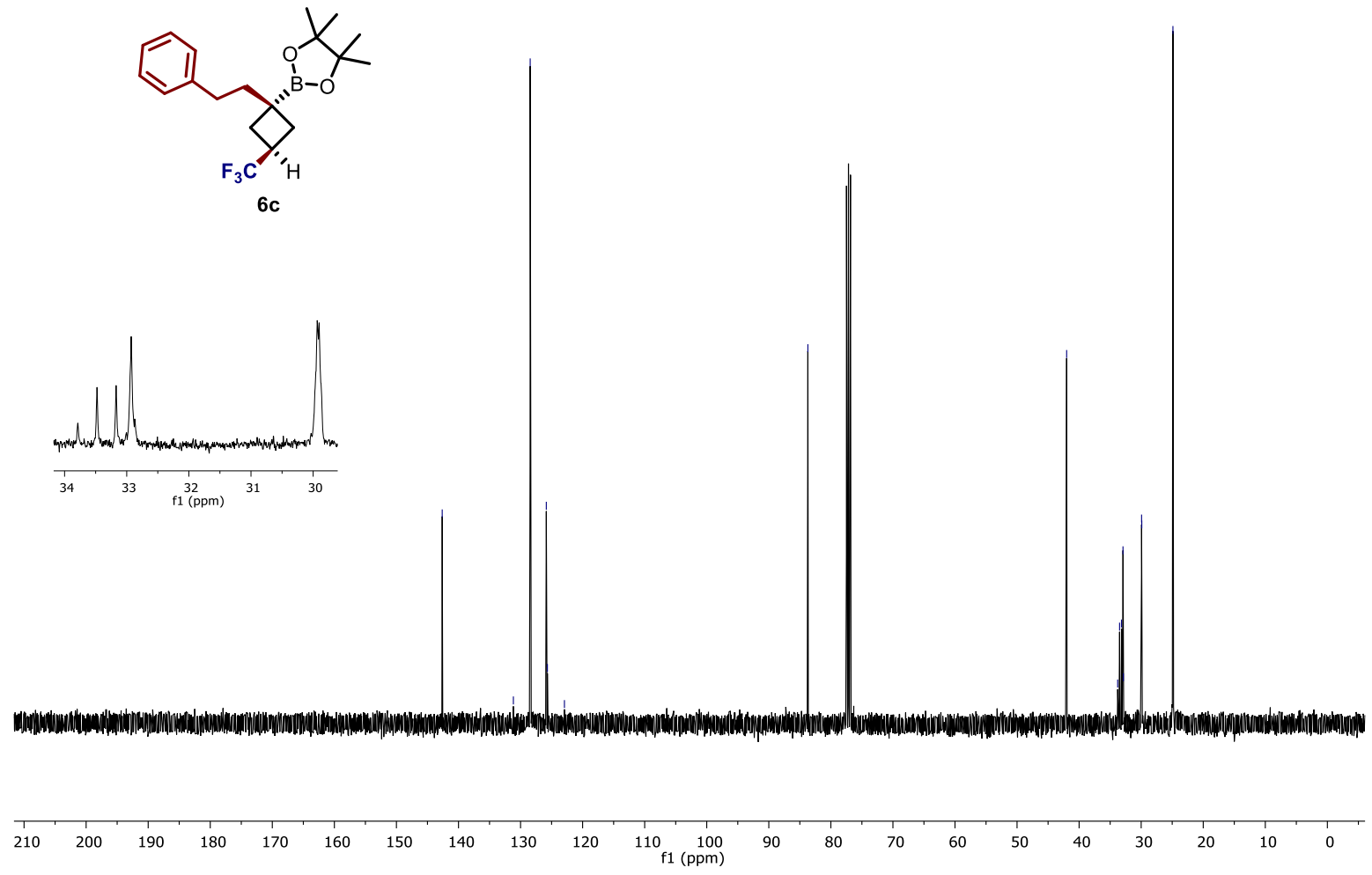

S48 
${ }^{19} \mathrm{~F}$ NMR of compound $\mathbf{6 c}\left(377 \mathrm{MHz}, \mathrm{CDCl}_{3}\right)$

$\mathrm{va} / \mathrm{ms} 78565$ MSP747 19F_single_pulse
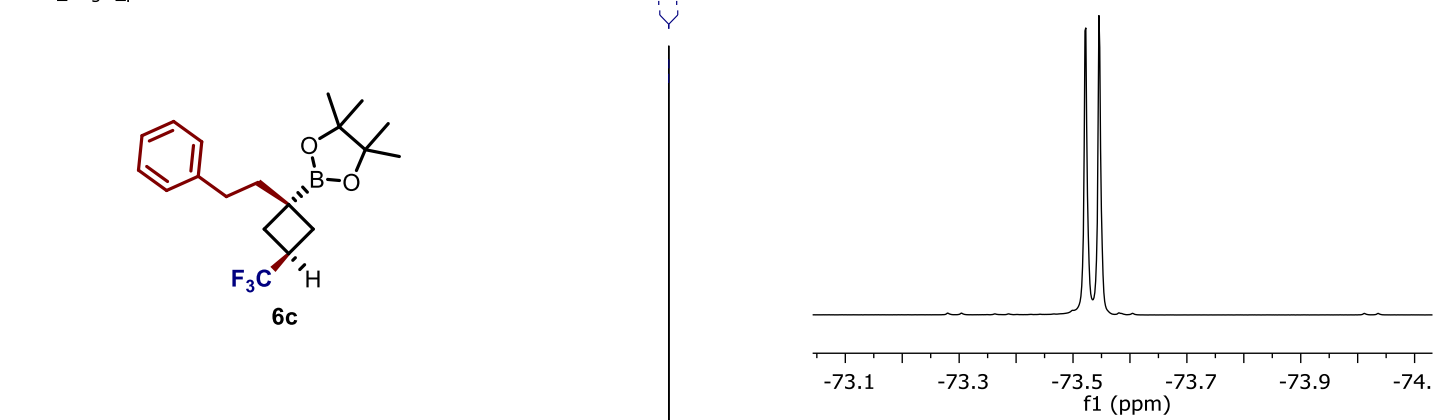

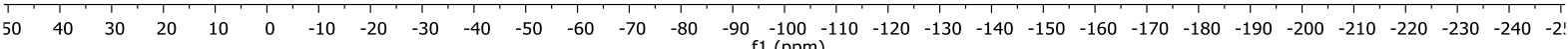


${ }^{1} \mathrm{H}$ NMR of compound $\mathbf{6 d}\left(500 \mathrm{MHz}, \mathrm{CDCl}_{3}\right)$

ms17103_MSP755p_PROTON01
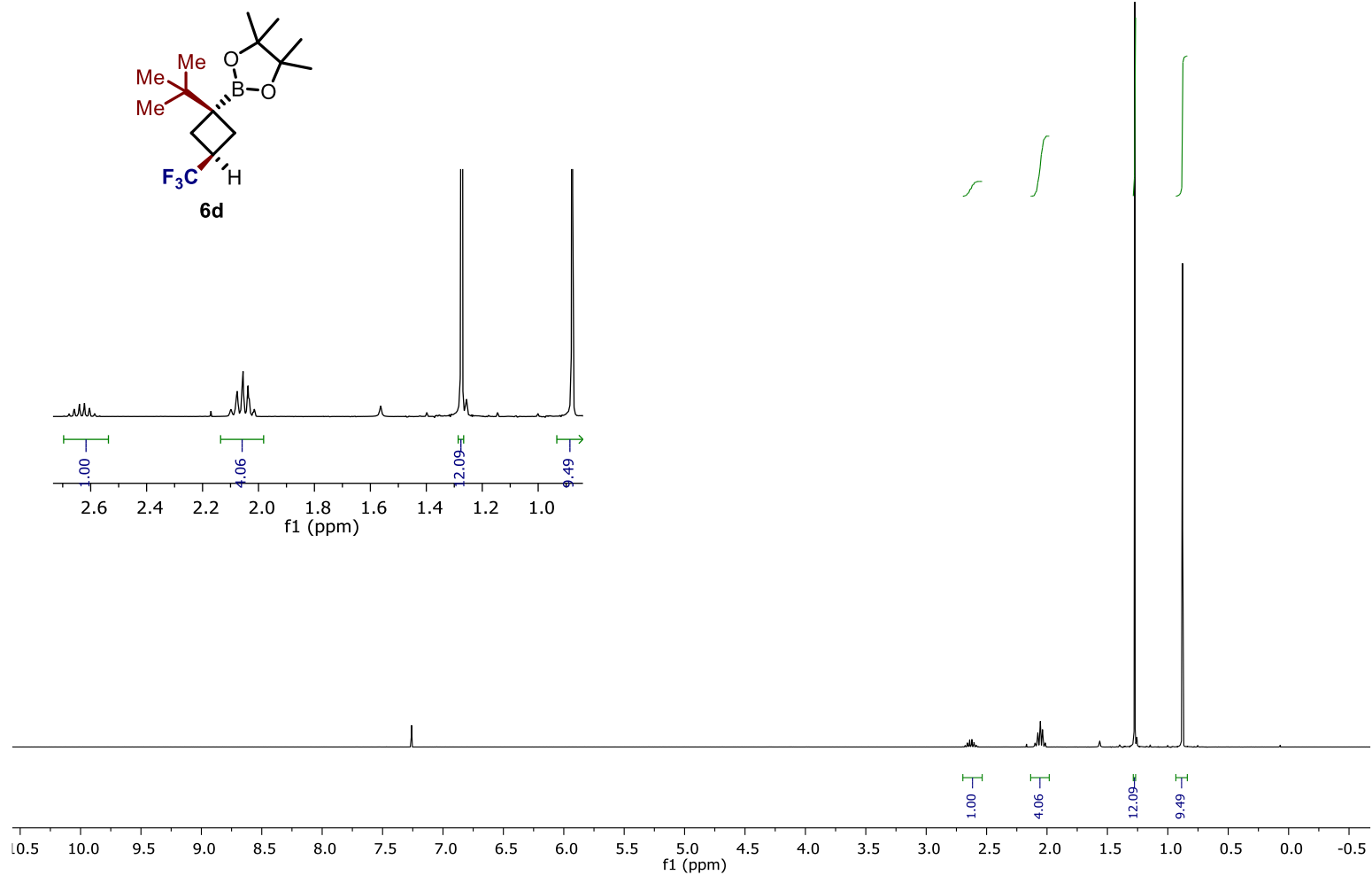

${ }^{13} \mathrm{C}$ NMR of compound $\mathbf{6 d}\left(125 \mathrm{MHz}, \mathrm{CDCl}_{3}\right)$

ms17103_MSP755p_CARBONO

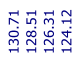

111

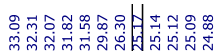

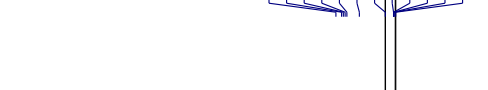

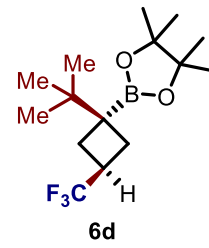

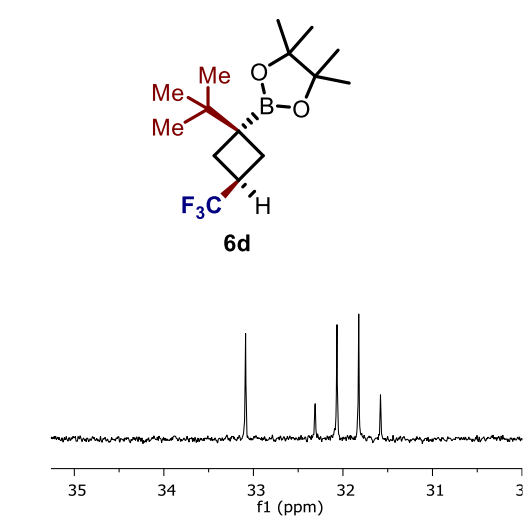

$\begin{array}{lllllllllll}200 & 190 & 180 & 170 & 160 & 150 & 140 & 130 & 120 & 110 & \begin{array}{c}100 \\ \mathrm{f} 1(\mathrm{ppm})\end{array}\end{array}$ 
${ }^{19} \mathrm{~F}$ NMR of compound $\mathbf{6 d}\left(377 \mathrm{MHz}, \mathrm{CDCl}_{3}\right)$

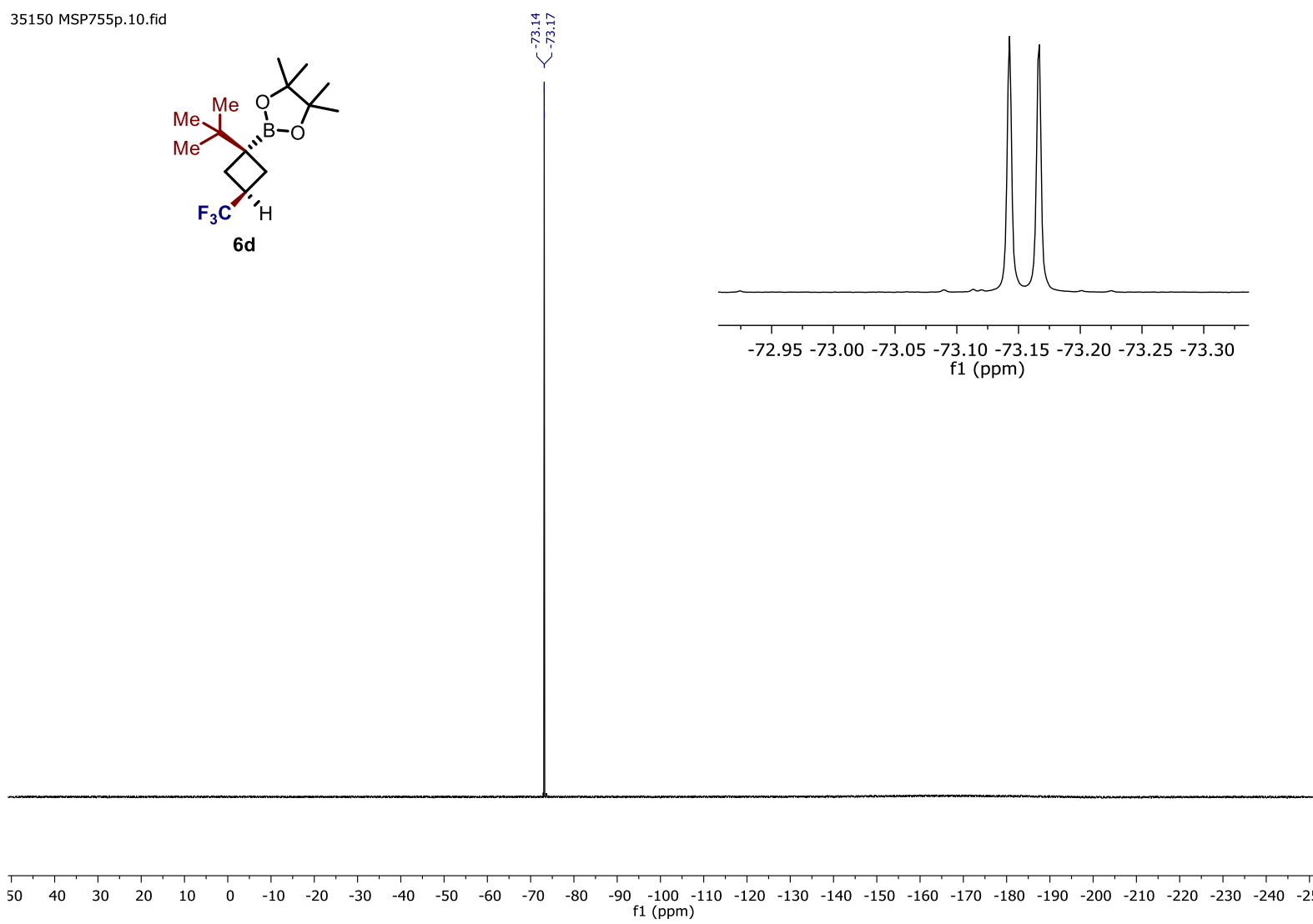


${ }^{1} \mathrm{H}$ NMR of compound $6 \mathbf{e}\left(500 \mathrm{MHz}, \mathrm{CDCl}_{3}\right)$

35432 MSP762p.10.fid
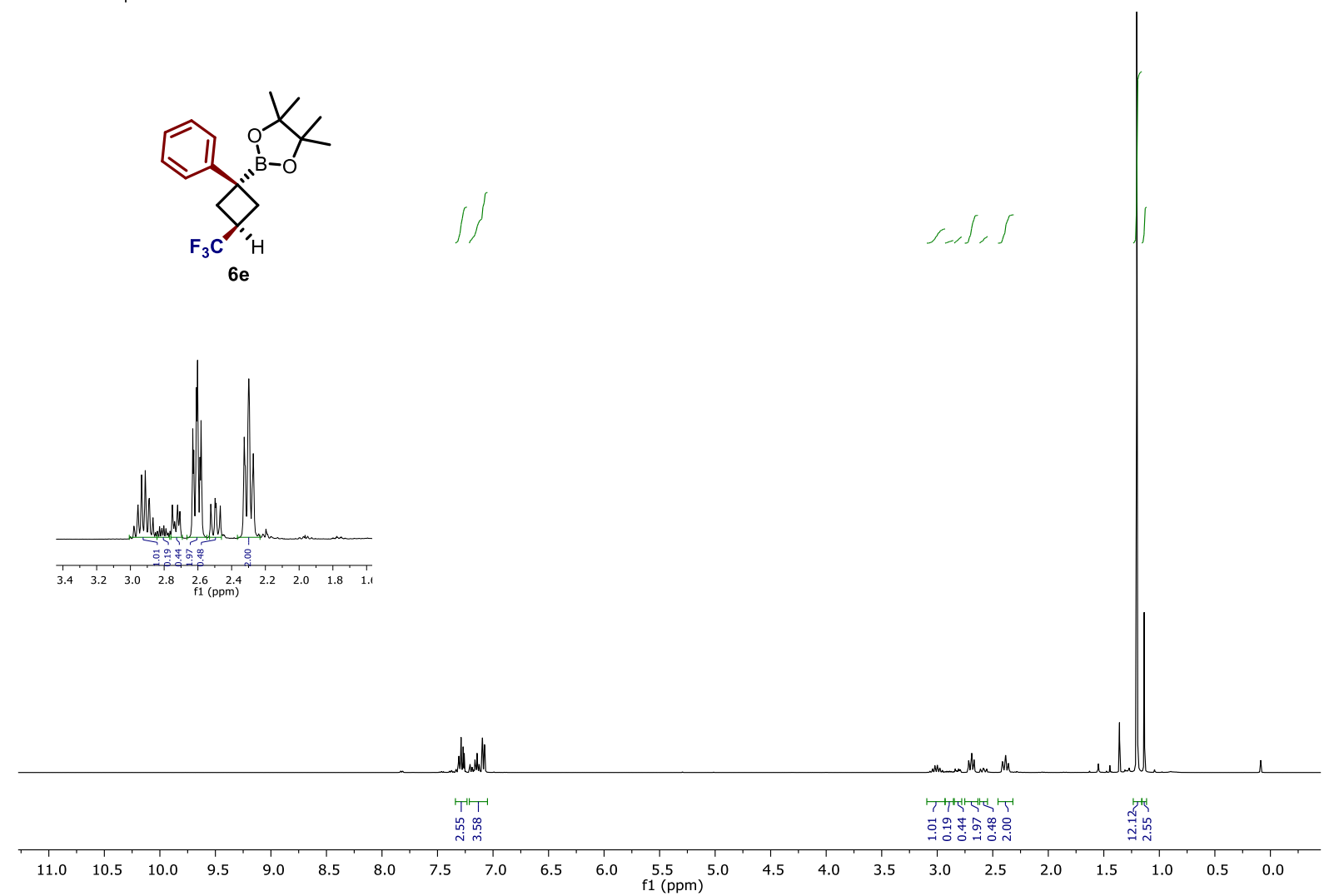

${ }^{13} \mathrm{C}$ NMR of compound $6 \mathbf{e}\left(125 \mathrm{MHz}, \mathrm{CDCl}_{3}\right)$

35432 MSP762p.12.fid
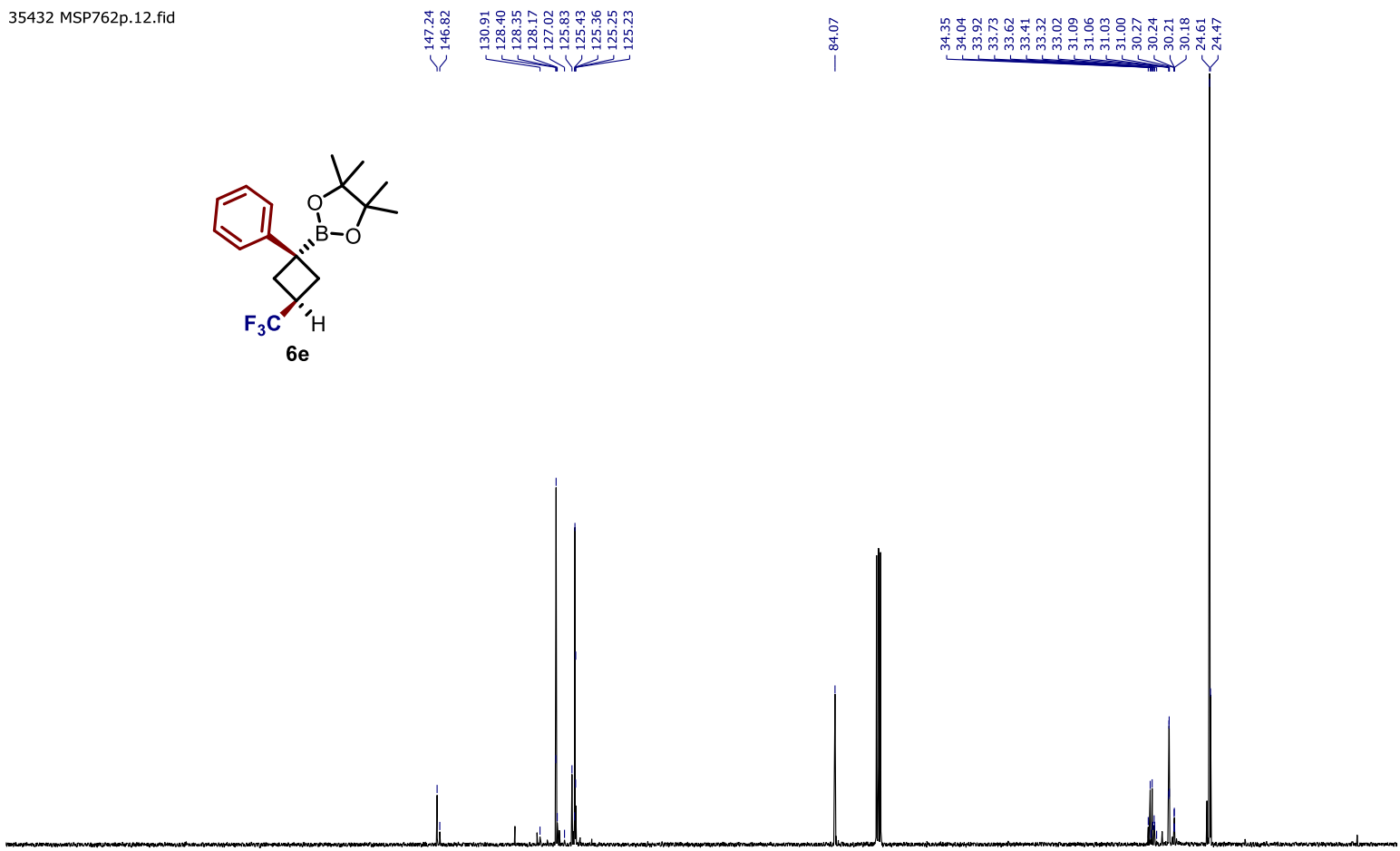

$\begin{array}{llllllllllll}210 & 200 & 190 & 180 & 170 & 160 & 150 & 140 & 130 & 120 & 110 & 100\end{array}$ 
${ }^{19} \mathrm{~F}$ NMR of compound $6 \mathbf{e}\left(377 \mathrm{MHz}, \mathrm{CDCl}_{3}\right)$

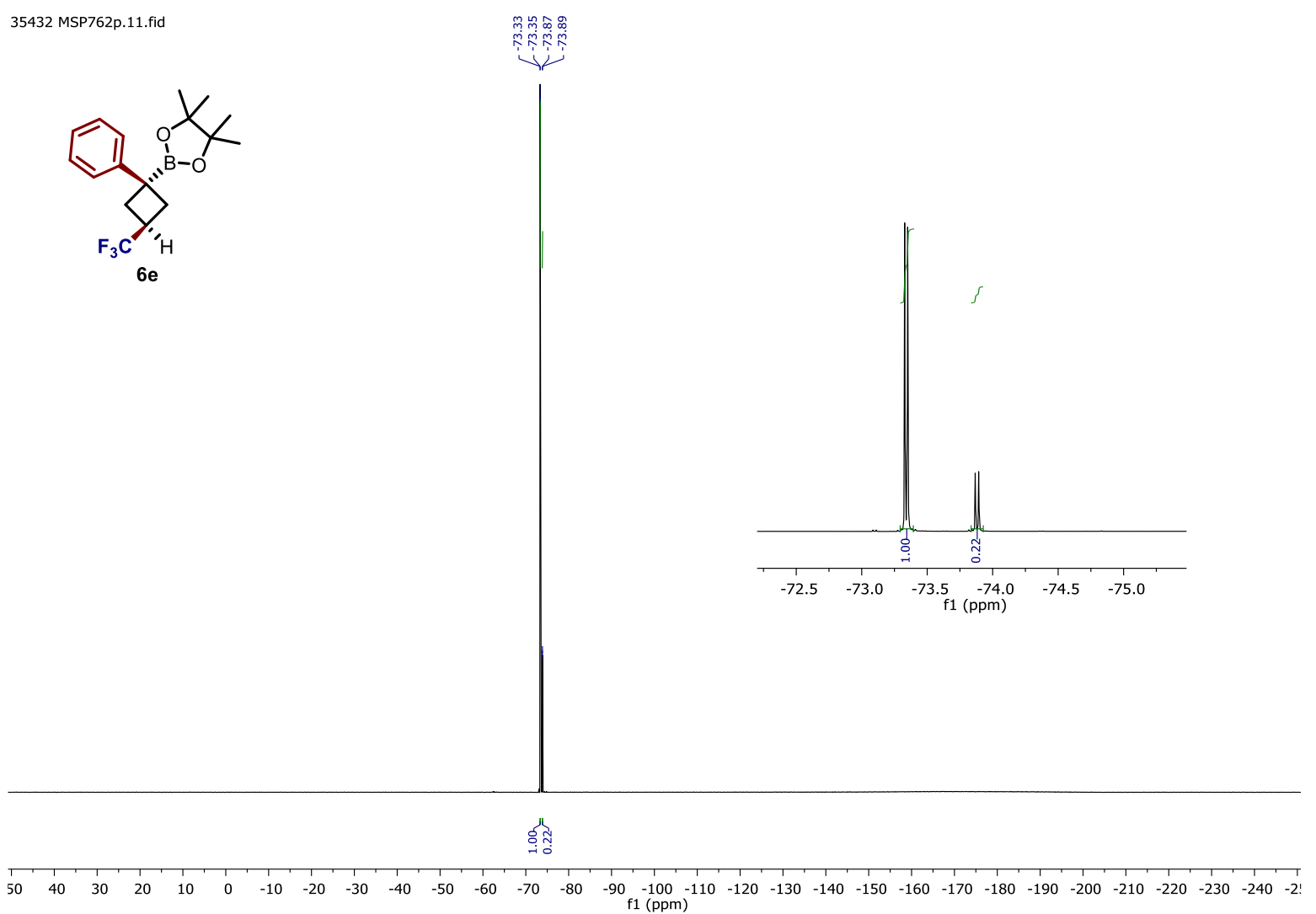


${ }^{1} \mathrm{H} \mathrm{NMR}$ of compound $\mathbf{6} \mathbf{f}\left(500 \mathrm{MHz}, \mathrm{CDCl}_{3}\right)$

ms45358_MSP763p_PROTON_01<smiles>[CH]C([CH])CC1(c2ccc(OC)cc2)OC(C)(C)O1</smiles>
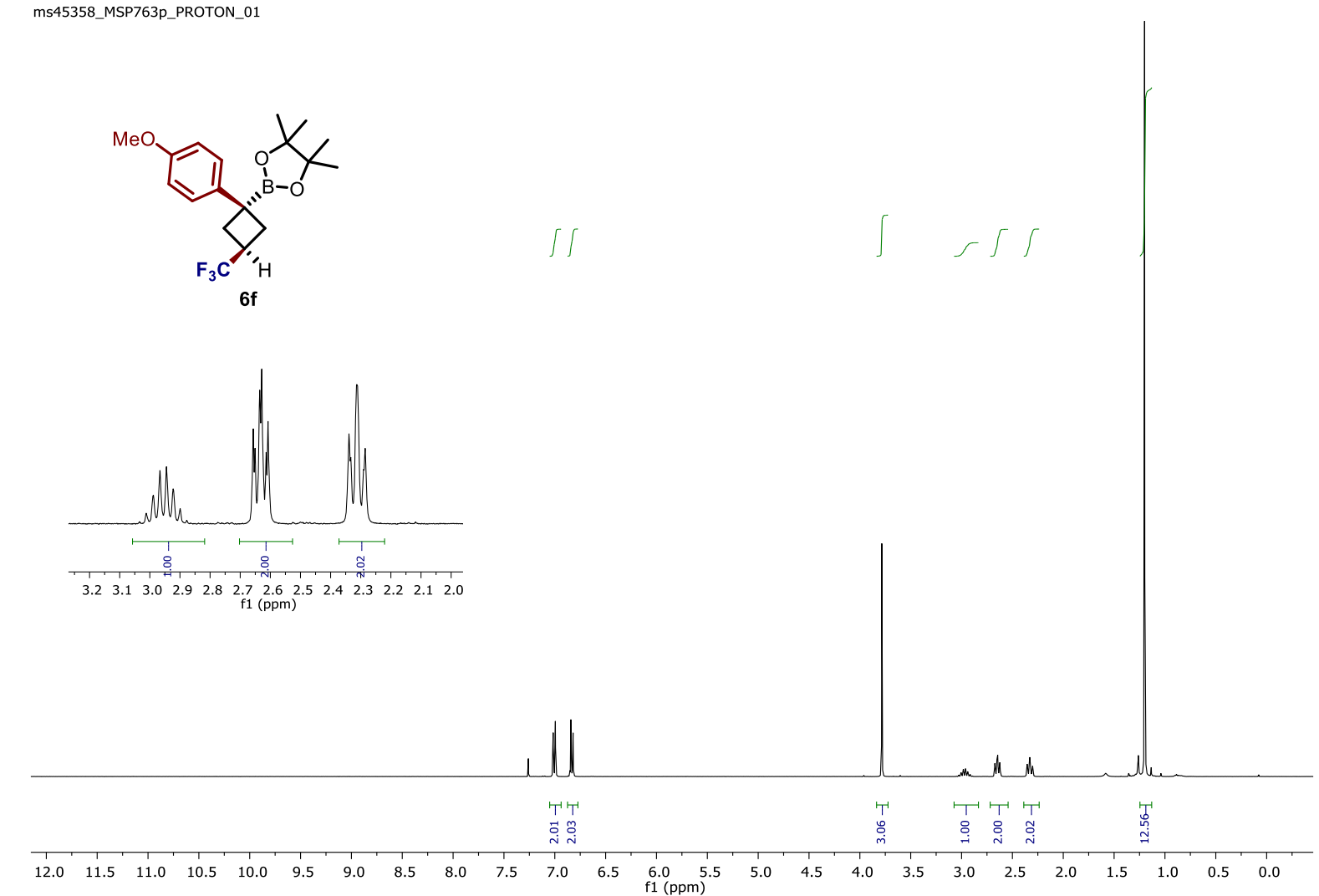

${ }^{13} \mathrm{C}$ NMR of compound $\mathbf{6 f}\left(125 \mathrm{MHz}, \mathrm{CDCl}_{3}\right)$

ms27039_MSP763p_CARBON_001
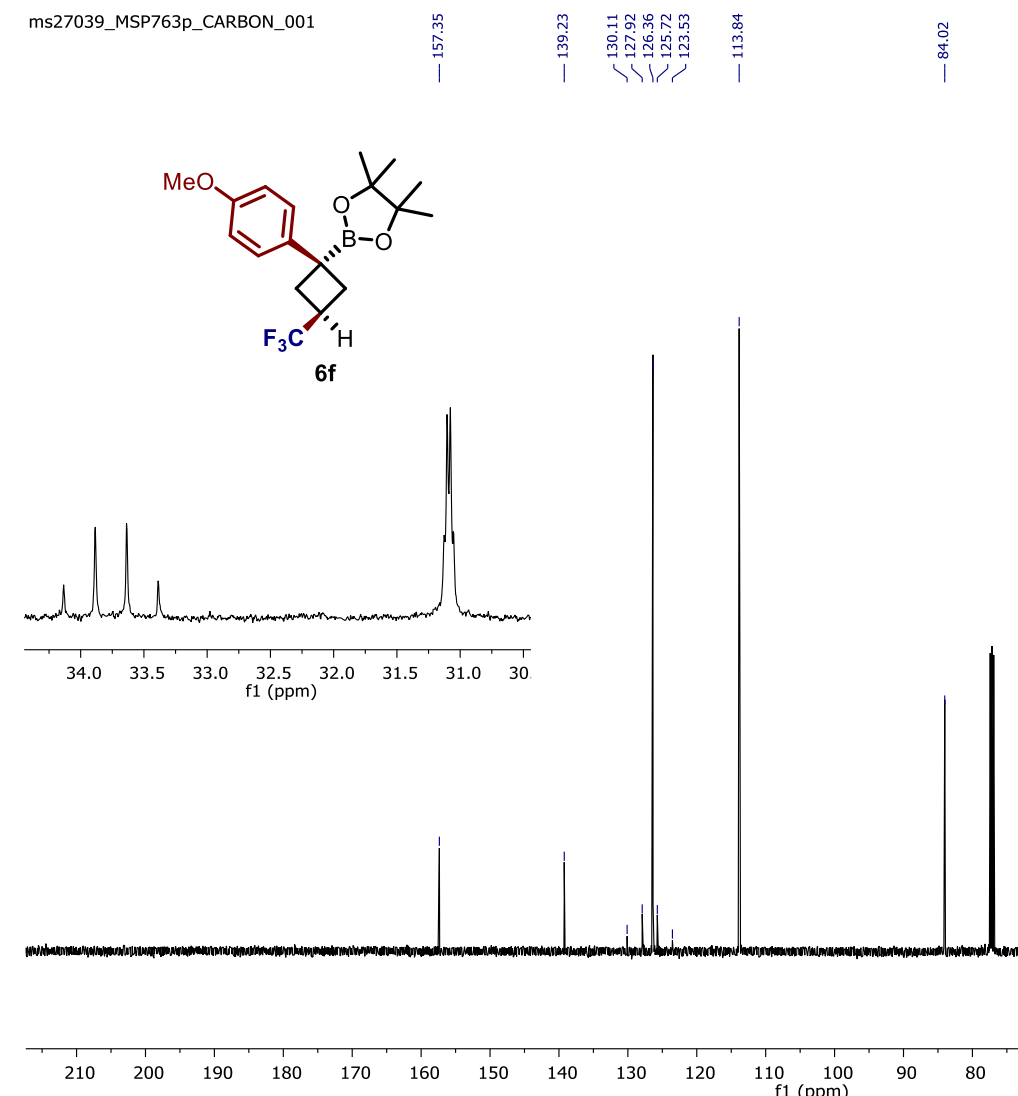

60

50

30 
${ }^{19} \mathrm{~F}$ NMR of compound $\mathbf{6 f}\left(470 \mathrm{MHz}, \mathrm{CDCl}_{3}\right)$

ms27039_MSP763P_FLUORINE_001 TIANDARD PROTON PARAMETERS
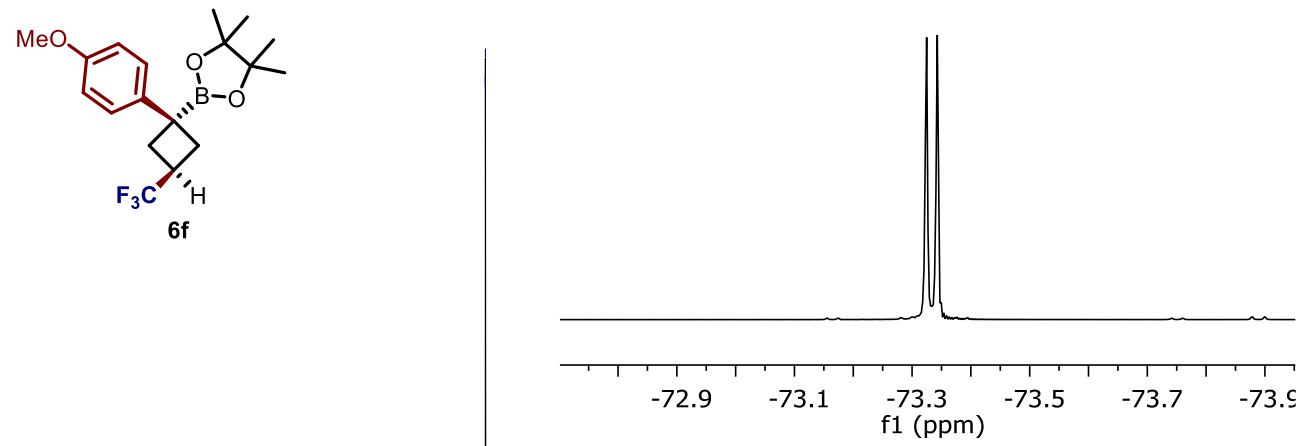

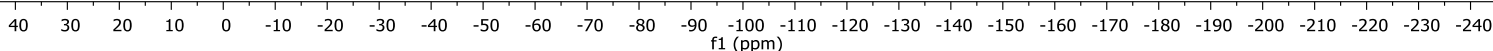


${ }^{1} \mathrm{H}$ NMR of compound $\mathbf{6 g}\left(500 \mathrm{MHz}, \mathrm{CDCl}_{3}\right)$

36195 MSP766_P2.10.fid
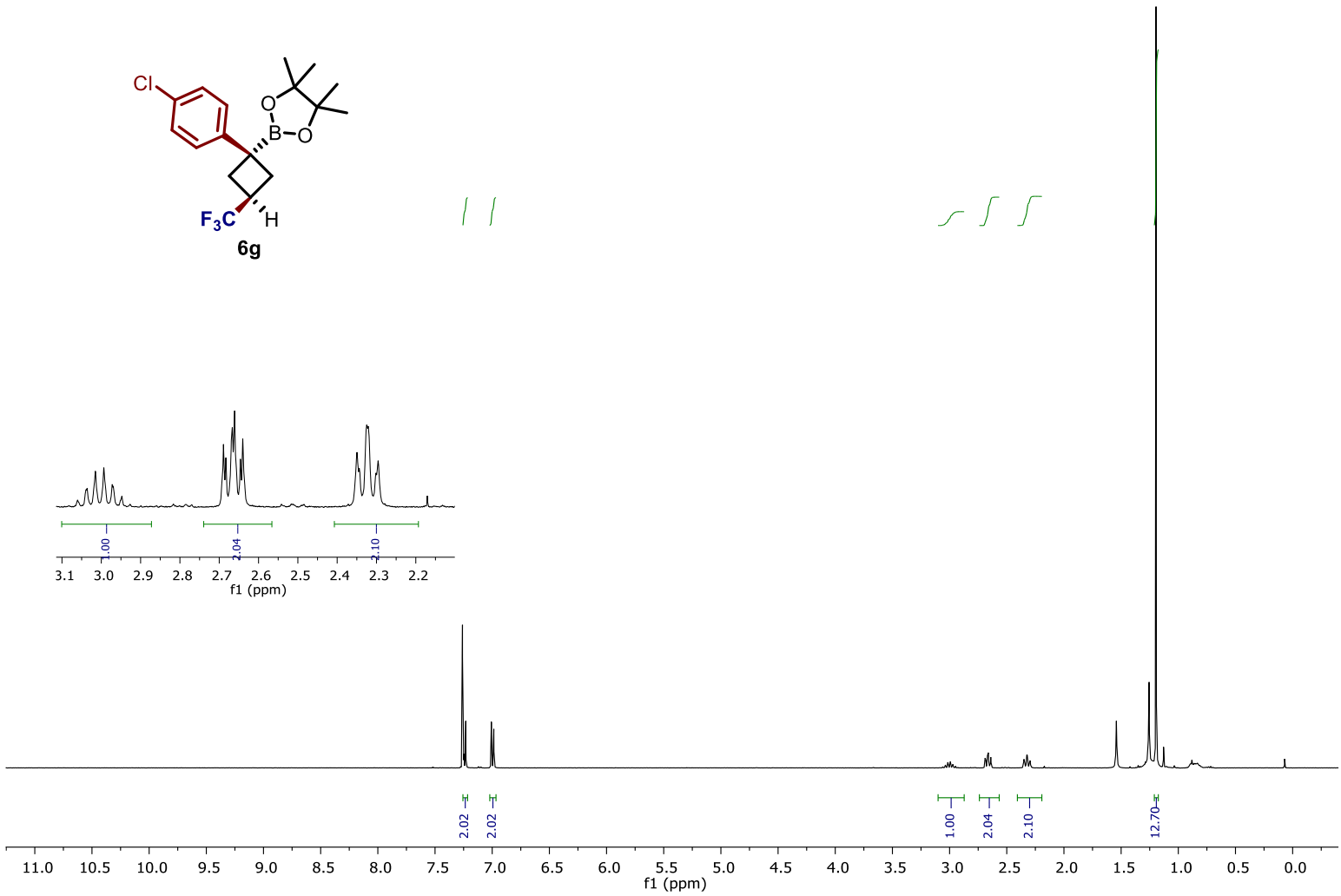

${ }^{13} \mathrm{C}$ NMR of compound $\mathbf{6 g}\left(125 \mathrm{MHz}, \mathrm{CDCl}_{3}\right)$

36156 MSP766p.12.fid
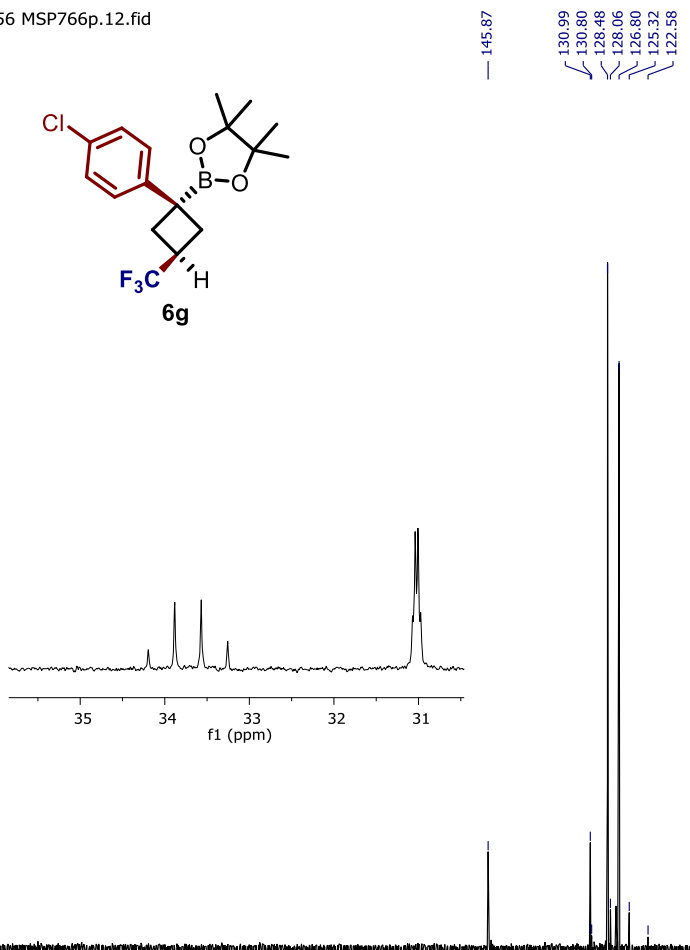

$220 \quad 210 \quad 200$
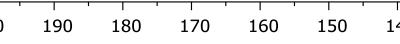

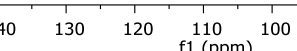

80

$50 \quad 40$ 
${ }^{19} \mathrm{~F}$ NMR of compound $\mathbf{6 g}\left(470 \mathrm{MHz}, \mathrm{CDCl}_{3}\right)$

36195 MSP766_P2.11.fid

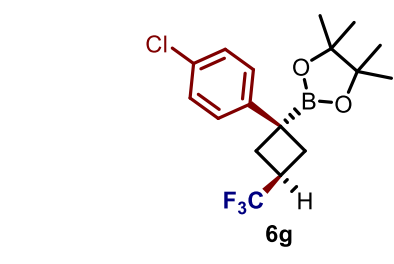

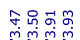

定

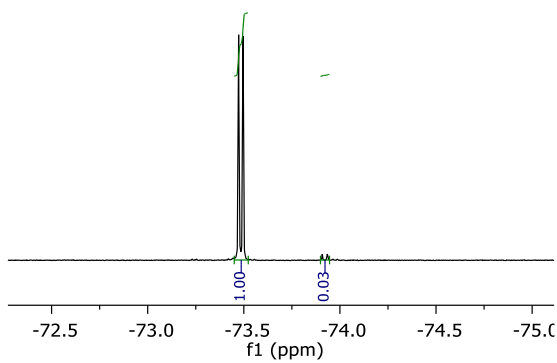

要

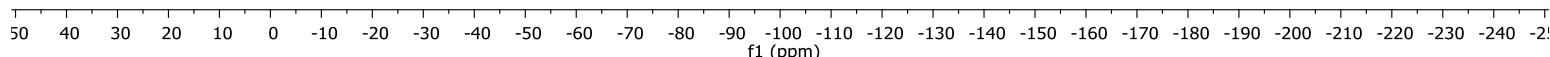


${ }^{1} \mathrm{H} \mathrm{NMR}$ of compound $\mathbf{6 h}\left(500 \mathrm{MHz}, \mathrm{CDCl}_{3}\right)$

ms17103_MSP798p
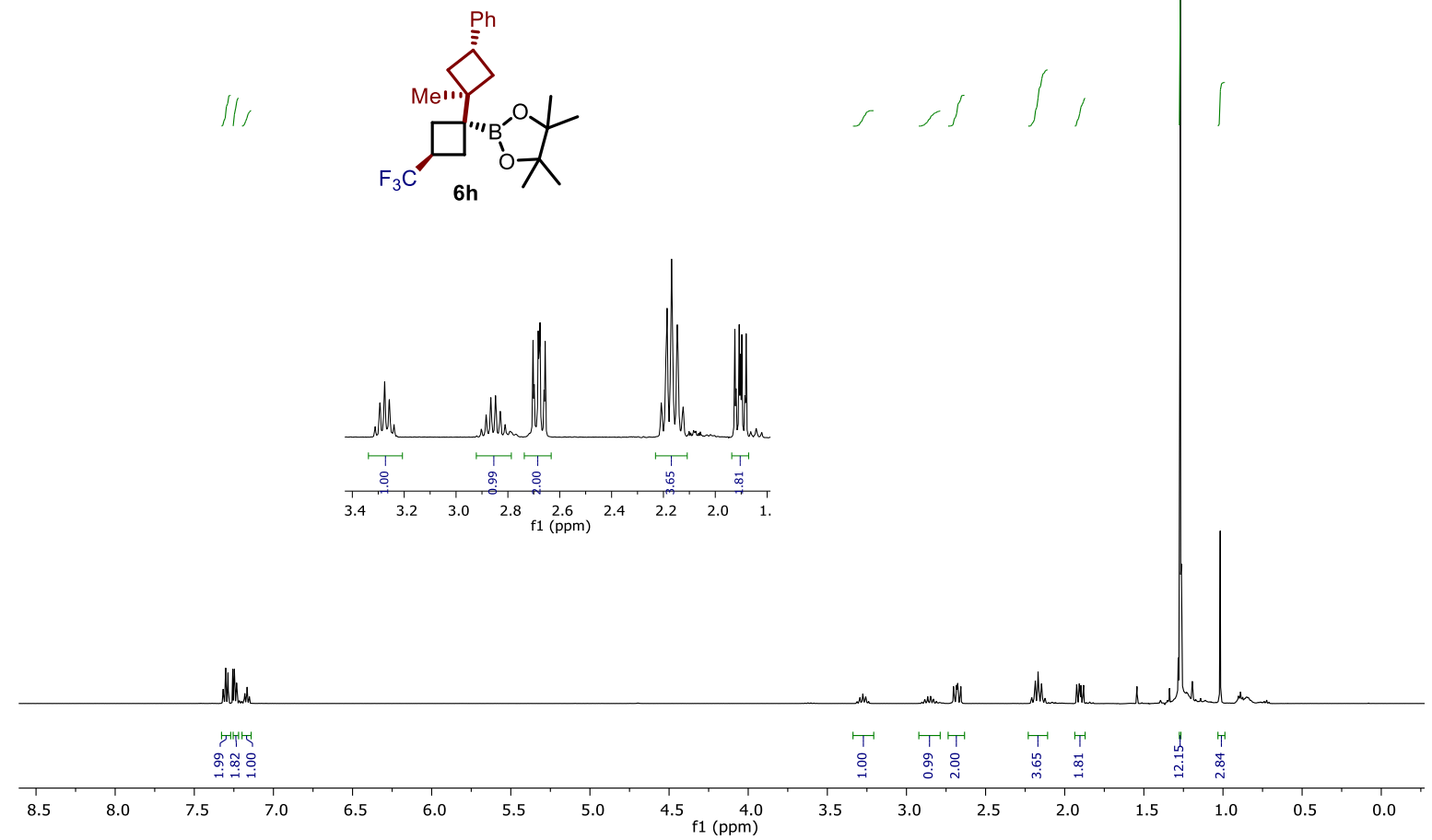

${ }^{13} \mathrm{C}$ NMR of compound $\mathbf{6 h}\left(125 \mathrm{MHz}, \mathrm{CDCl}_{3}\right)$

ms17103_MSP798p
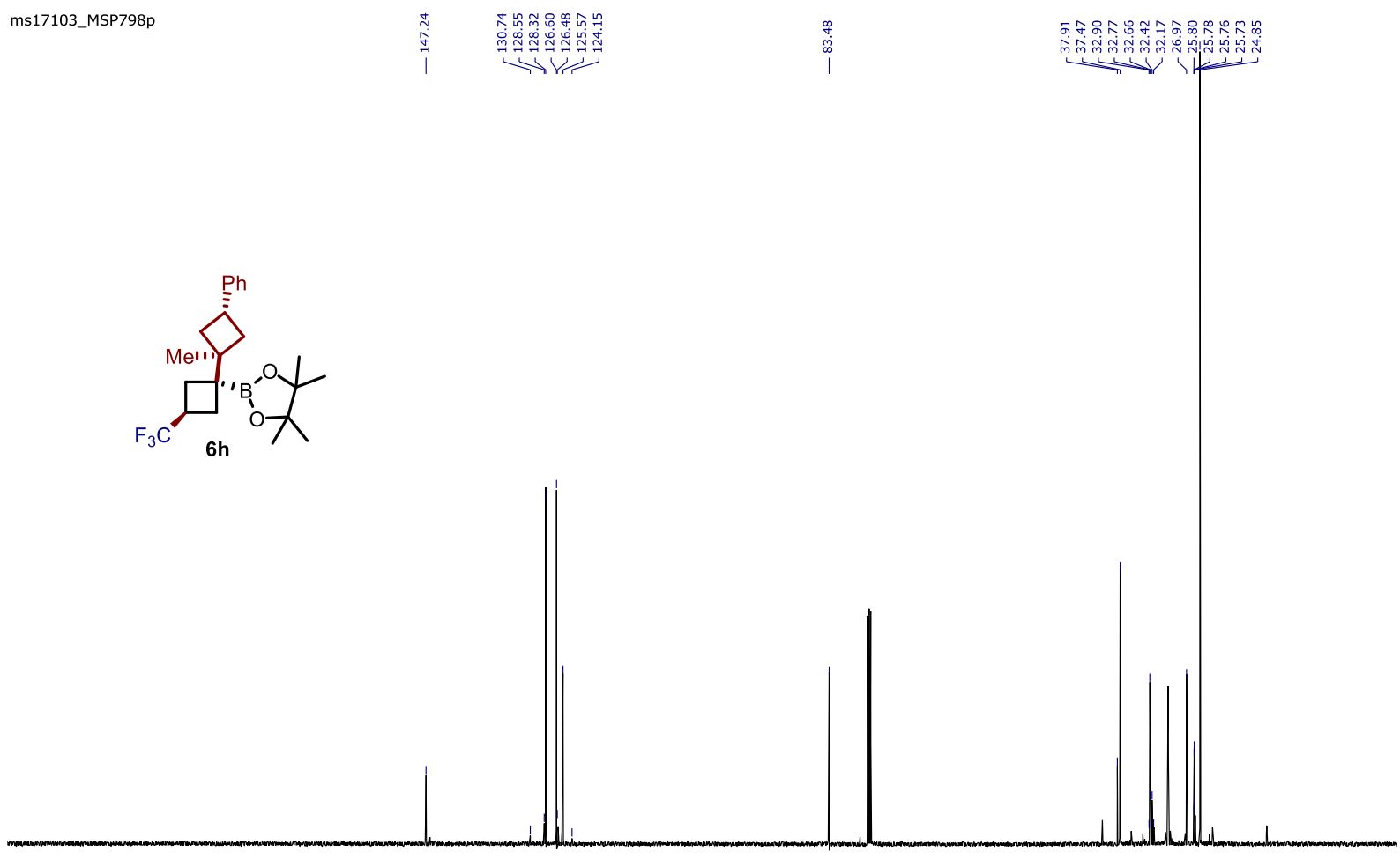

$\begin{array}{lllllllllll}210 & 200 & 190 & 180 & 170 & 160 & 150 & 140 & 130 & 120 & 110 \underset{\mathrm{f} 1(\mathrm{ppm})}{100}\end{array}$ 
${ }^{19} \mathrm{~F}$ NMR of compound $\mathbf{6 h}\left(376 \mathrm{MHz}, \mathrm{CDCl}_{3}\right)$

ms1056 MSP798p_Fluorin

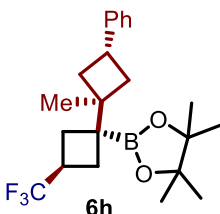

ำกำ

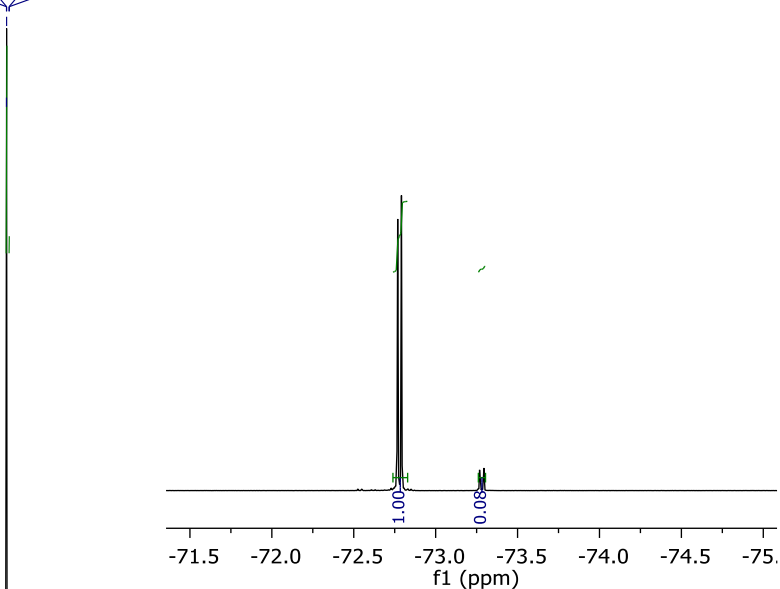

尊宫

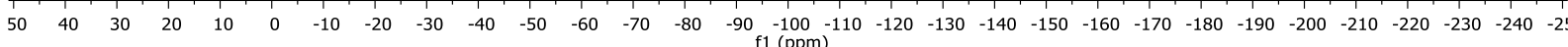


${ }^{1} \mathrm{H}$ NMR of compound $\mathbf{6} \mathbf{i}\left(400 \mathrm{MHz}, \mathrm{CDCl}_{3}\right)$

ms4286 MSP794p

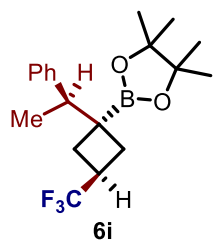
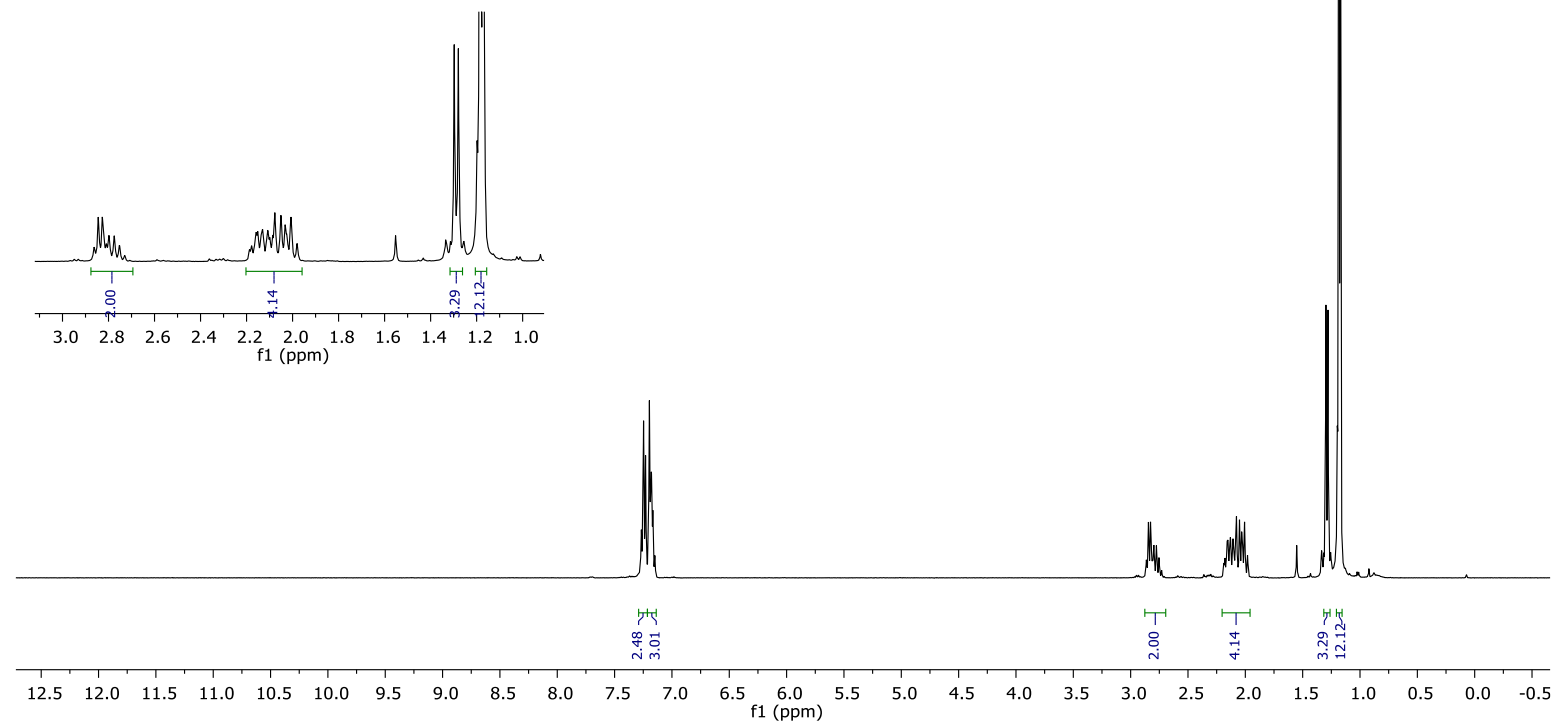

${ }^{13} \mathrm{C}$ NMR of compound $6 \mathbf{i}\left(100 \mathrm{MHz}, \mathrm{CDCl}_{3}\right)$

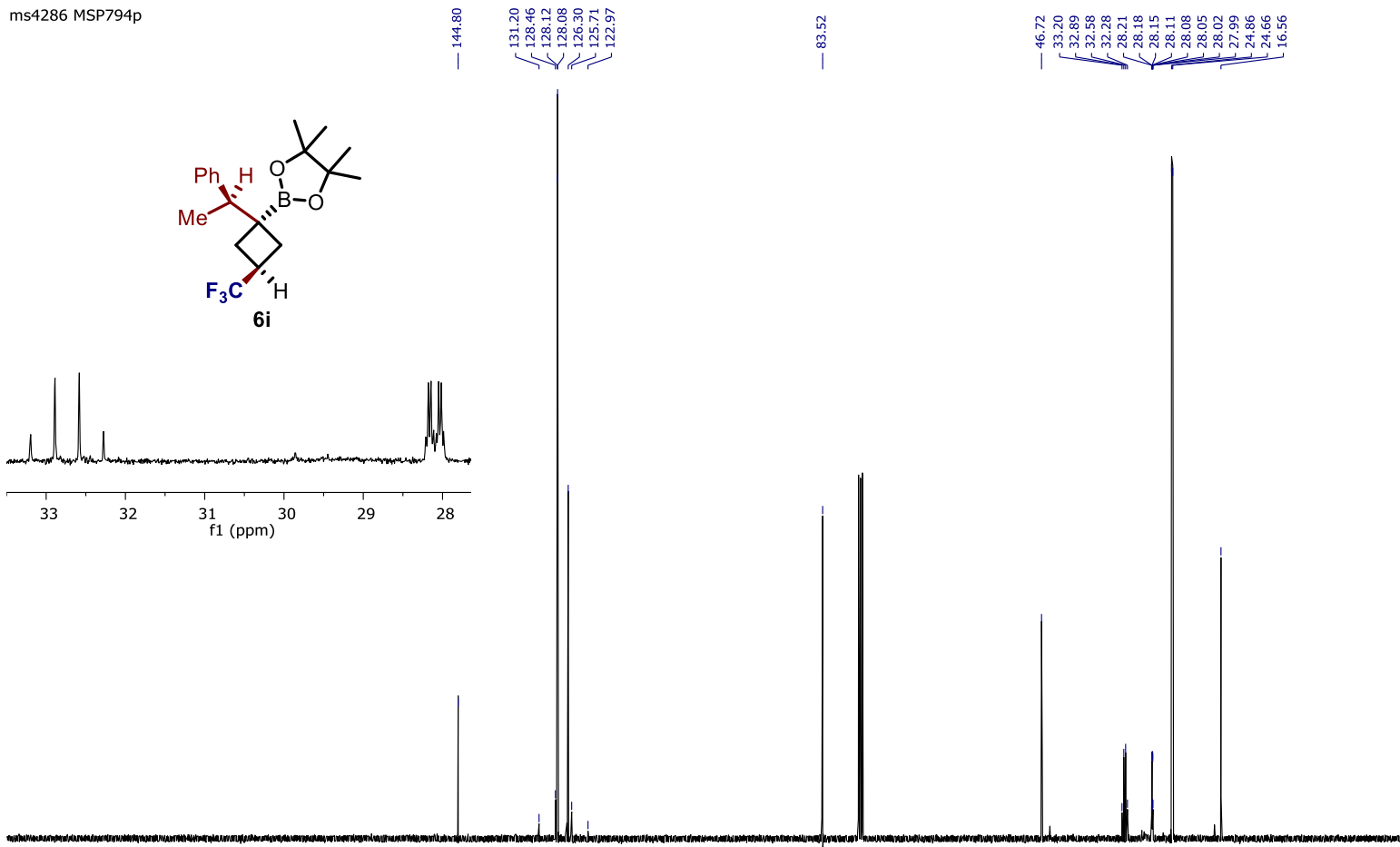

$\begin{array}{llllllllllllllllllllllllll}120 & 210 & 200 & 190 & 180 & 170 & 160 & 150 & 140 & 130 & 120 & 110 & 100 & 90 & 80 & 70 & 60 & 50 & 40 & 30 & 20 & 10 & 0 & -10\end{array}$ 
${ }^{19} \mathrm{~F}$ NMR of compound $\mathbf{6 i}\left(377 \mathrm{MHz}, \mathrm{CDCl}_{3}\right)$

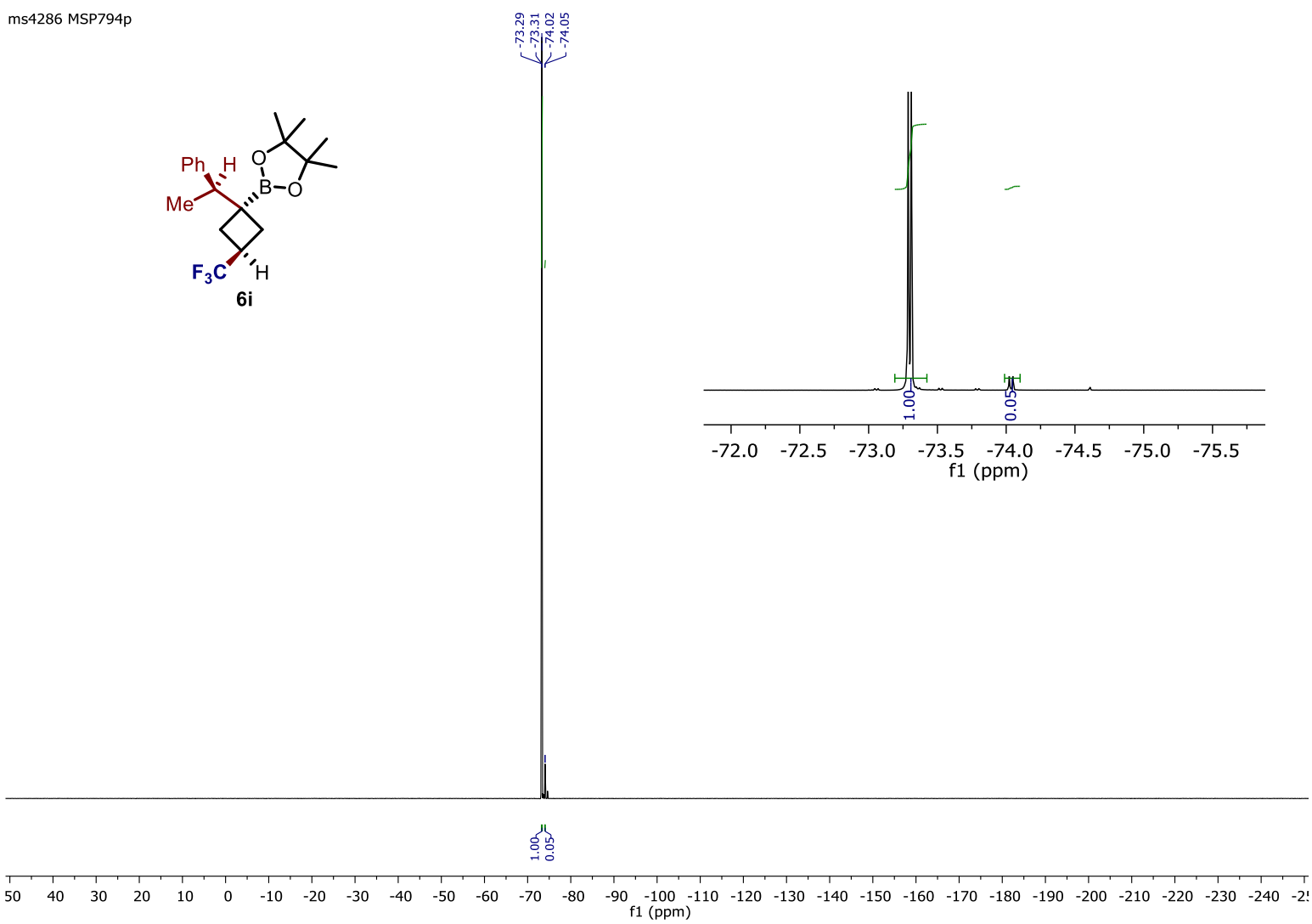


${ }^{1} \mathrm{H}$ NMR of compound $\mathbf{6 j}\left(500 \mathrm{MHz}, \mathrm{CDCl}_{3}\right.$ )

ms17102_MSP796P_PROTON01
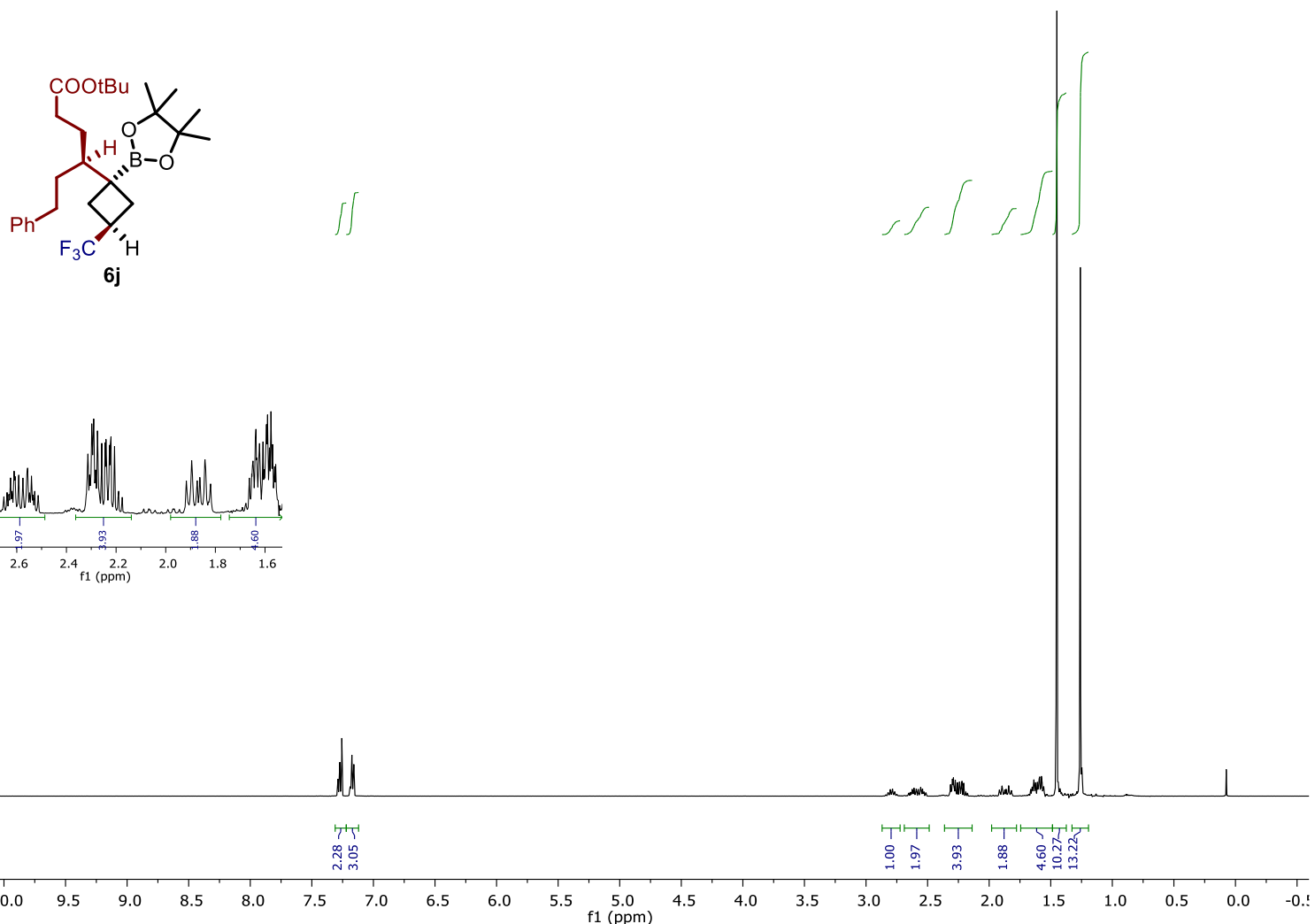

${ }^{13} \mathrm{C}$ NMR of compound $\mathbf{6 j}\left(125 \mathrm{MHz}, \mathrm{CDCl}_{3}\right)$

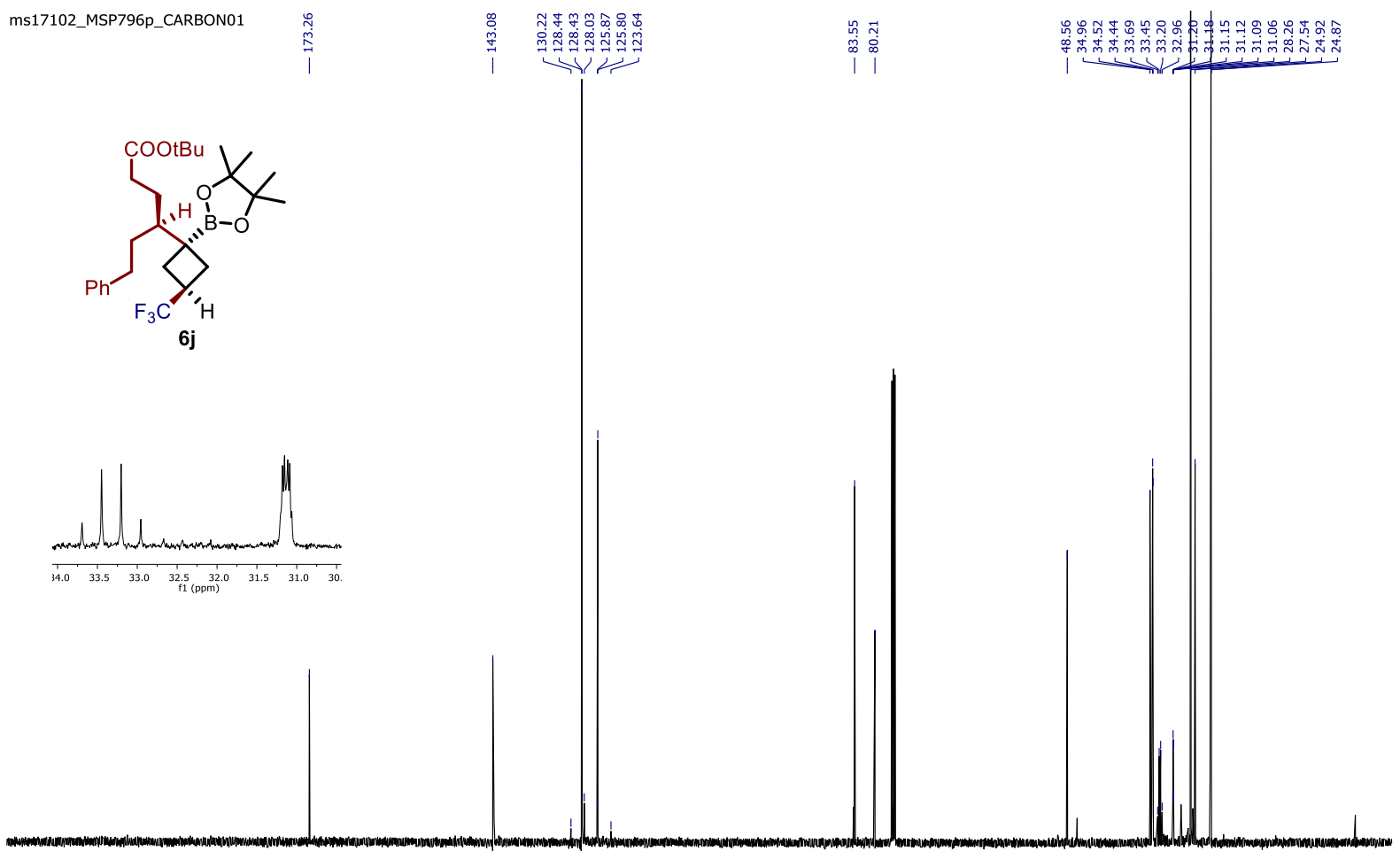

$\begin{array}{lllllllllllllllllllllllllllllll} & 220 & 210 & 200 & 190 & 180 & 170 & 160 & 150 & 140 & 130 & 120 & 110 & 100 & 90 & 80 & 70 & 60 & 50 & 40 & 30 & 20 & 10 & 0\end{array}$ 
${ }^{19} \mathrm{~F}$ NMR of compound $\mathbf{6 j}\left(377 \mathrm{MHz}, \mathrm{CDCl}_{3}\right.$ )

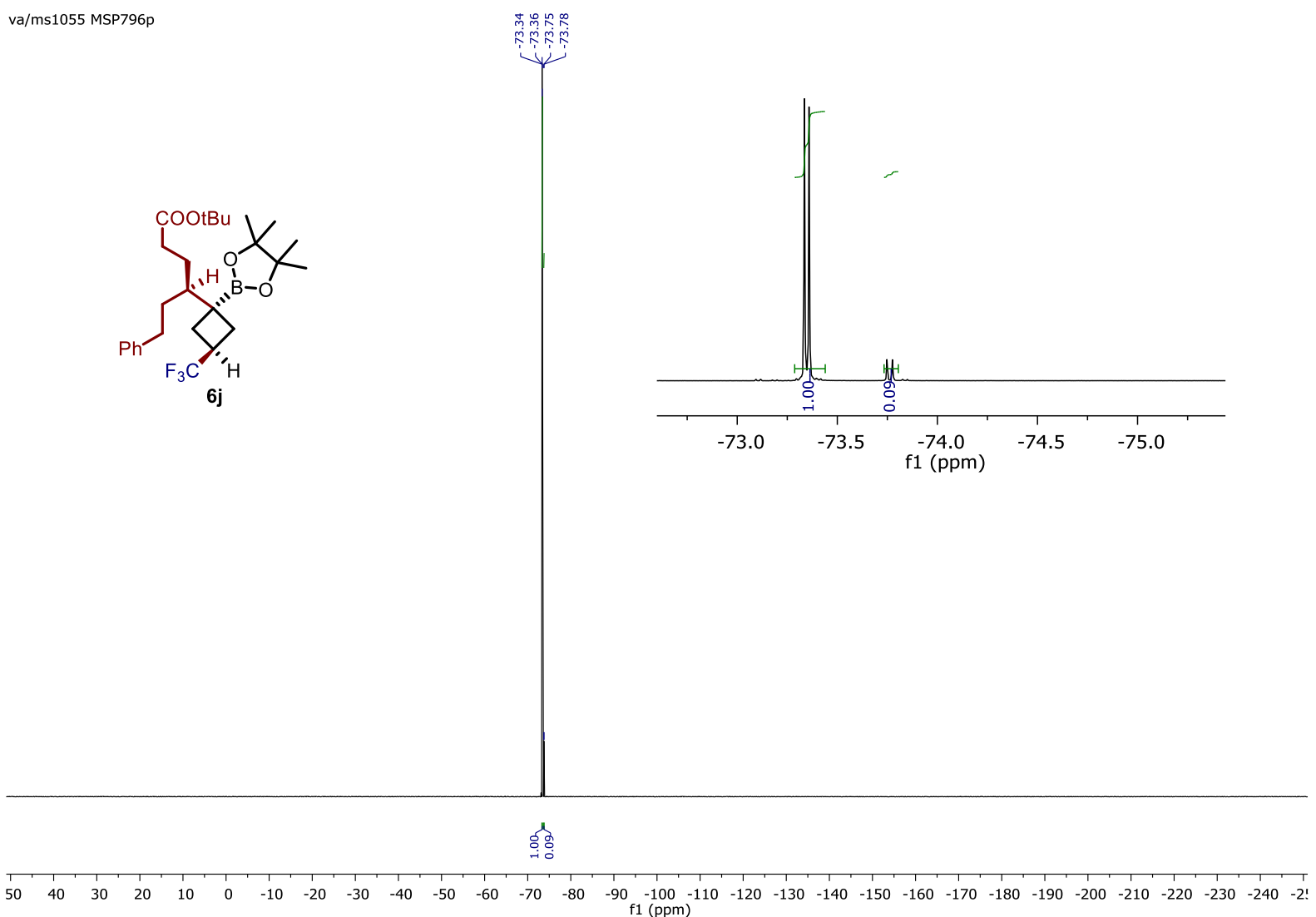


${ }^{1} \mathrm{H}$ NMR of compound $\mathbf{6 k}\left(400 \mathrm{MHz}, \mathrm{CDCl}_{3}\right)$

9573 MSP789p
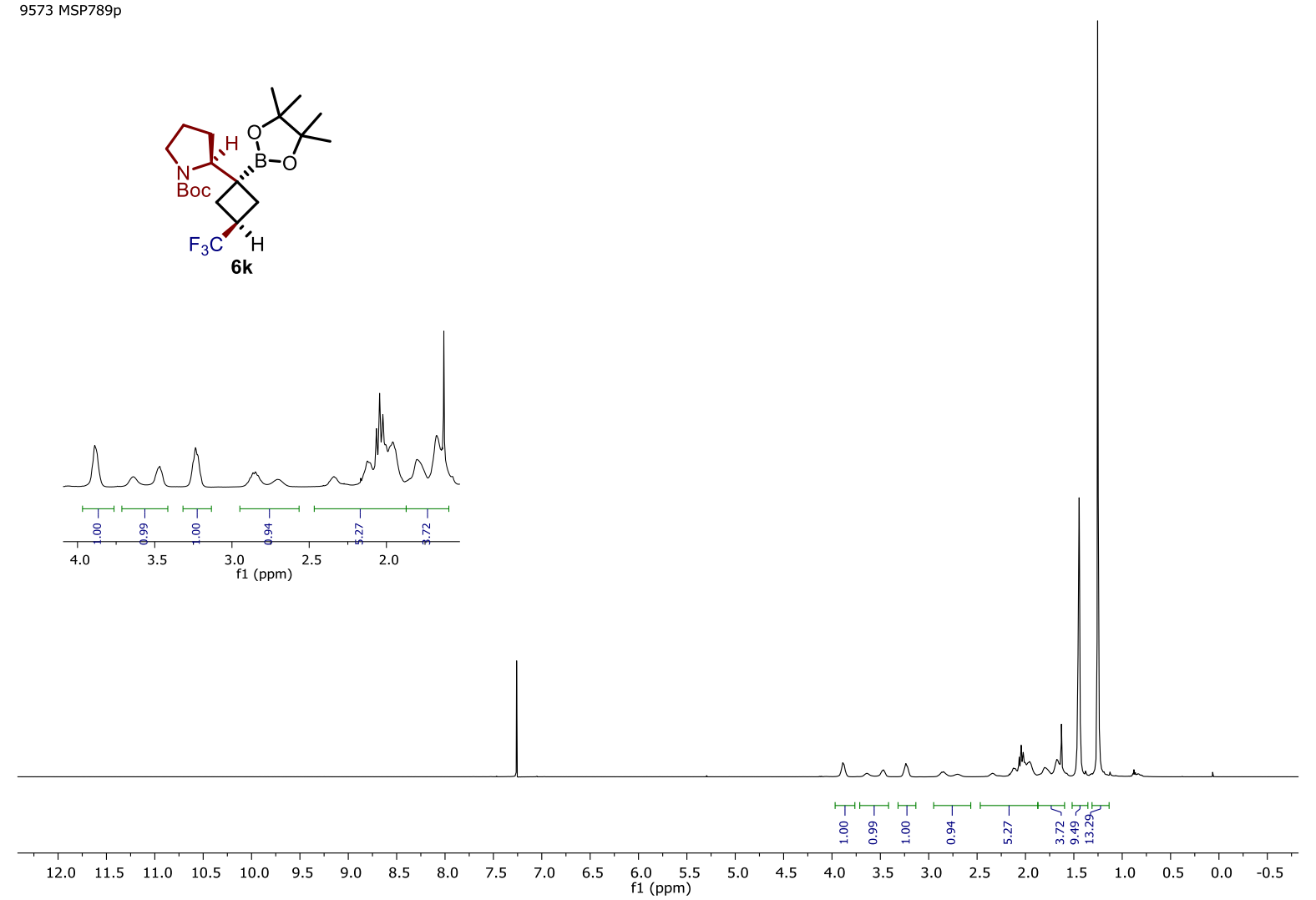

${ }^{13} \mathrm{C}$ NMR of compound $6 \mathbf{k}\left(125 \mathrm{MHz}, \mathrm{CDCl}_{3}\right)$

9573 MSP789p

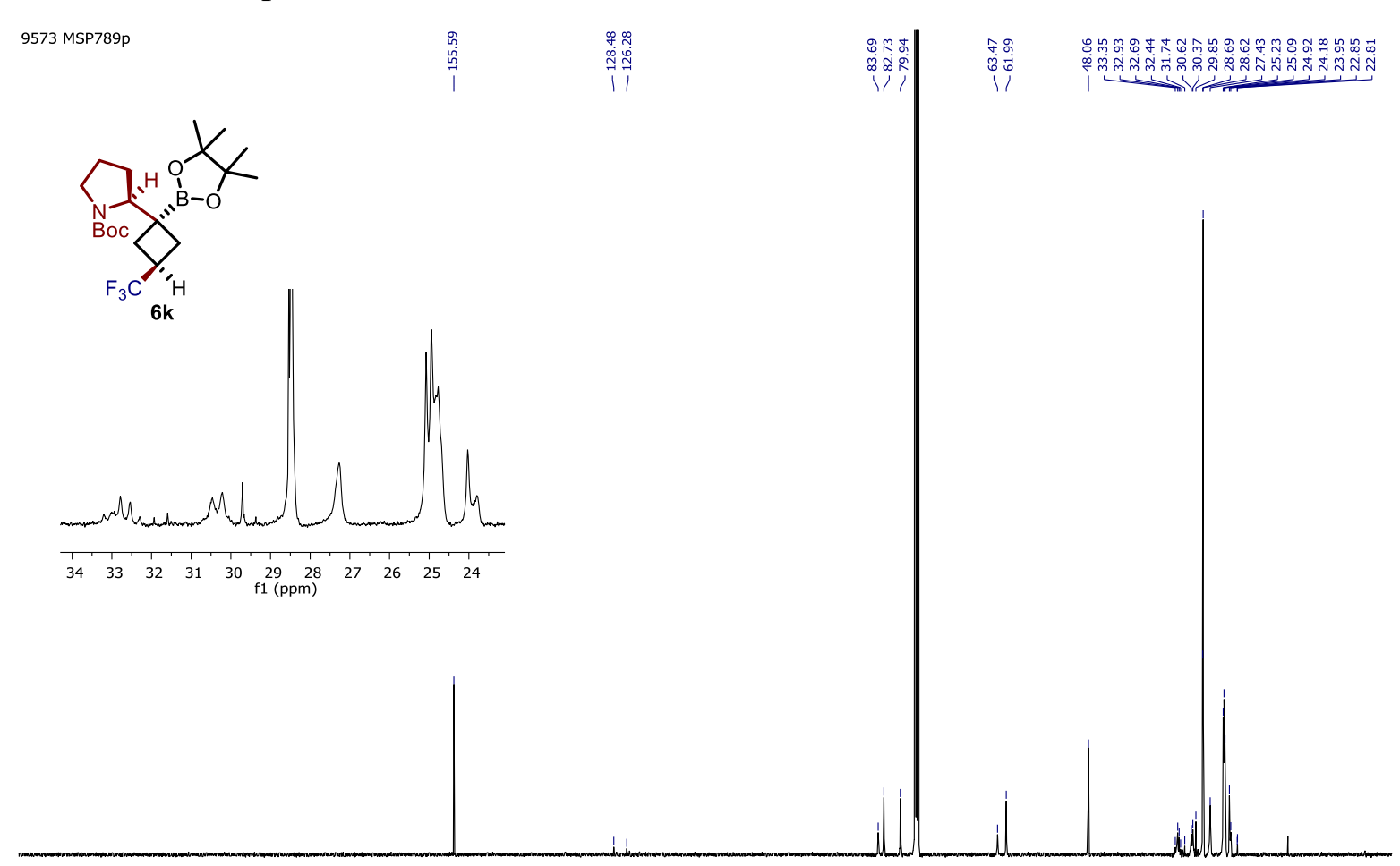

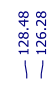

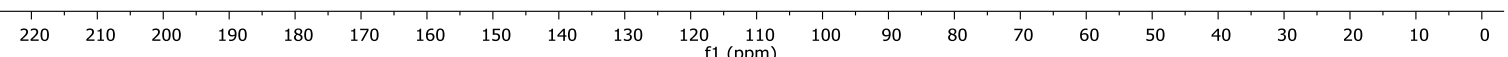


${ }^{19} \mathrm{~F}$ NMR of compound $\mathbf{6 k}\left(366 \mathrm{MHz}, \mathrm{CDCl}_{3}\right)$

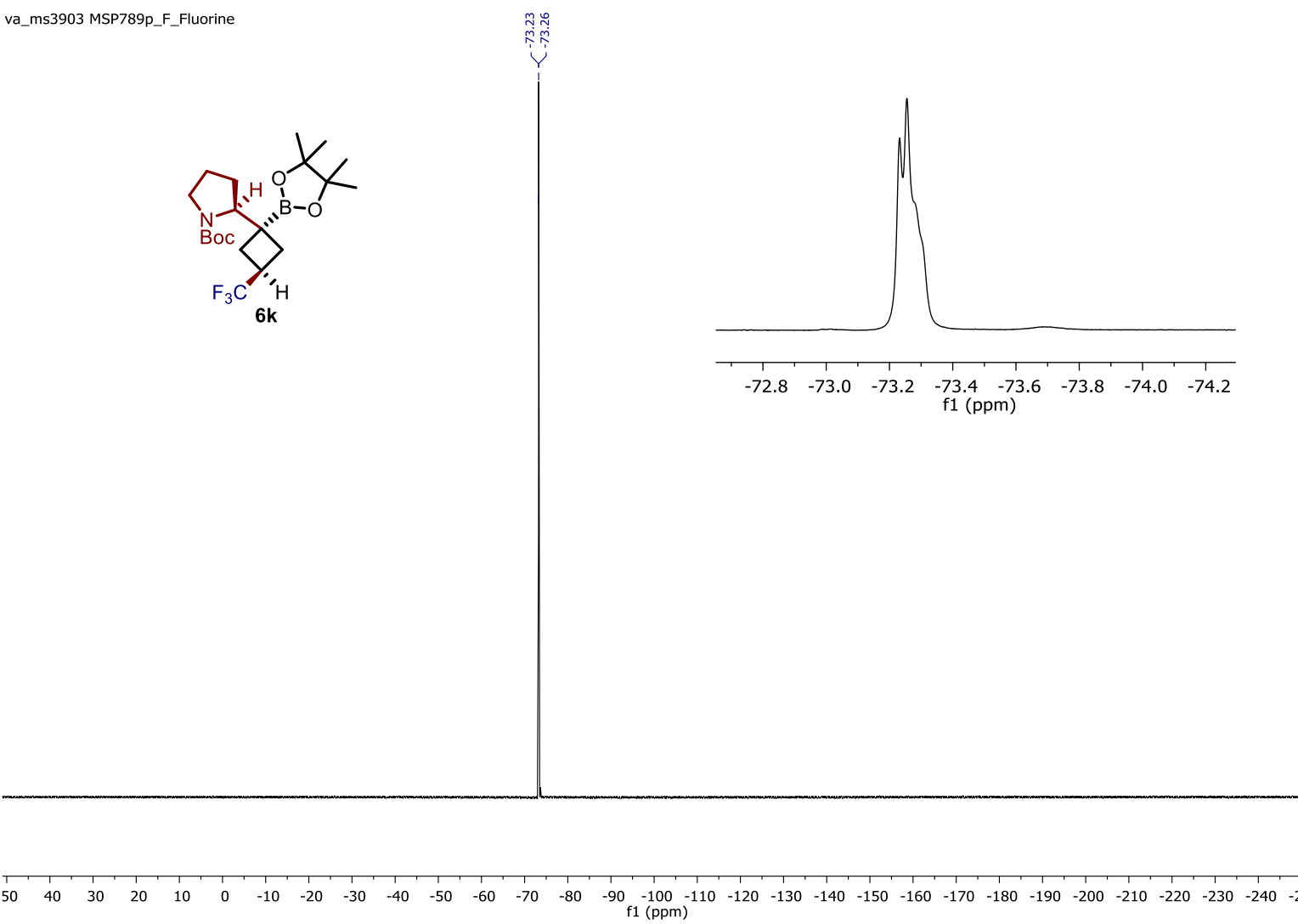


${ }^{1} \mathrm{H}$ NMR of compound 6k-1 (500 MHz, $\left.\mathrm{CDCl}_{3}\right)$

9617 MSP793p.10.fid

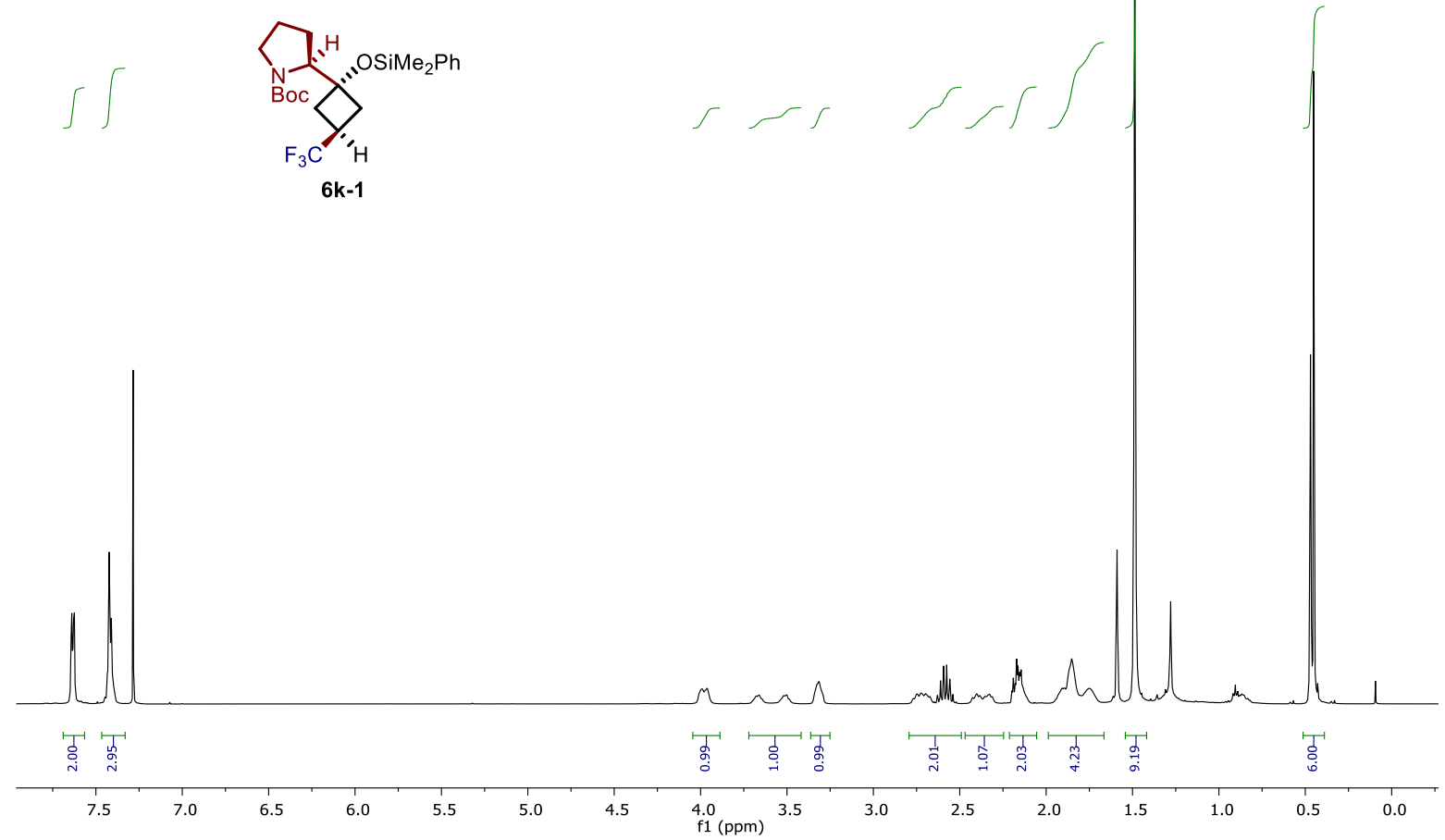

${ }^{1} \mathrm{H}$ NMR of compound 6k-1 (125 MHz, $\mathrm{CDCl}_{3}$ )

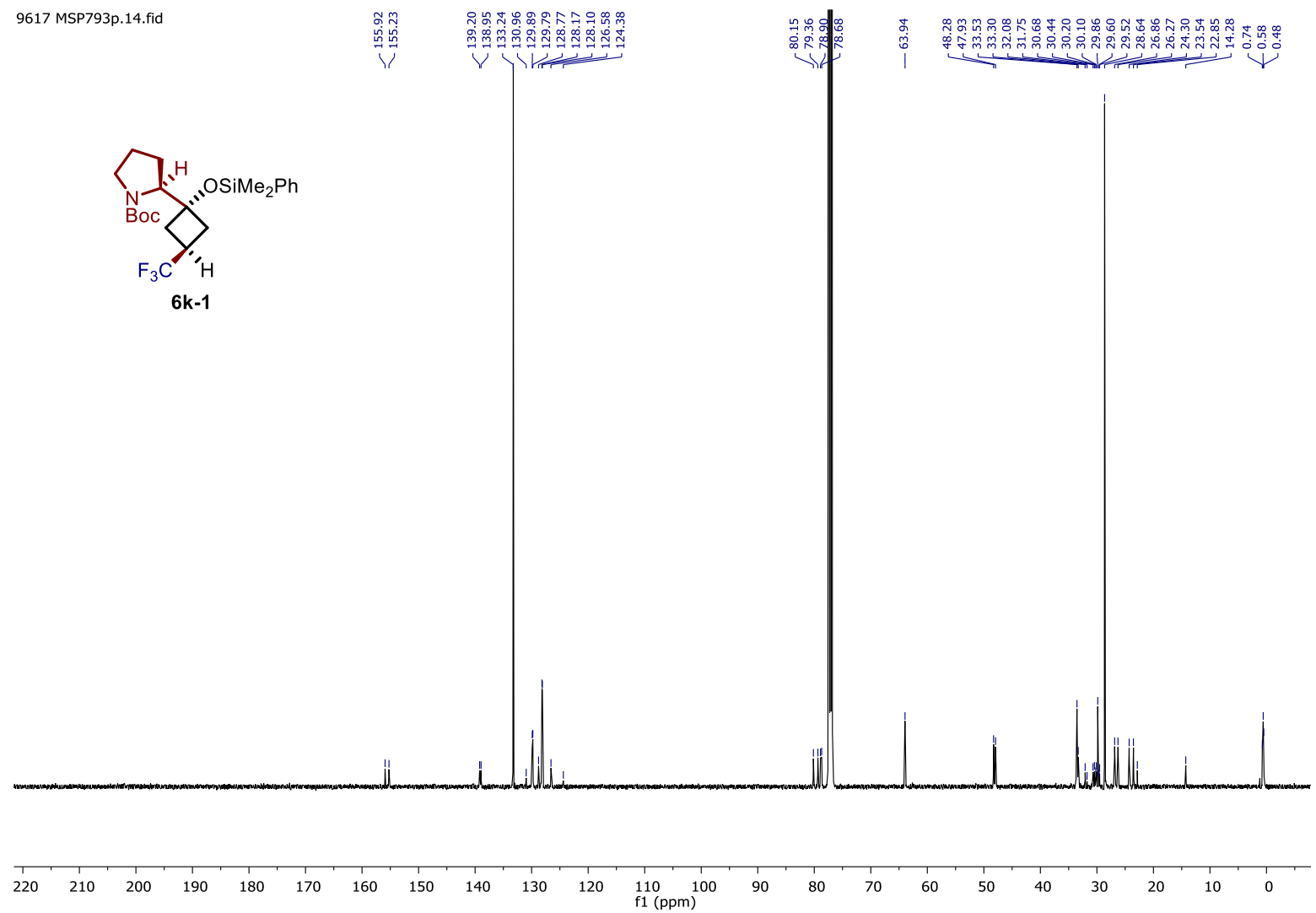


${ }^{19} \mathrm{~F}$ NMR of compound $\mathbf{6 k - 1}\left(470 \mathrm{MHz}, \mathrm{CDCl}_{3}\right)$

ms27380_MSP793P_FLUORINE_00 促
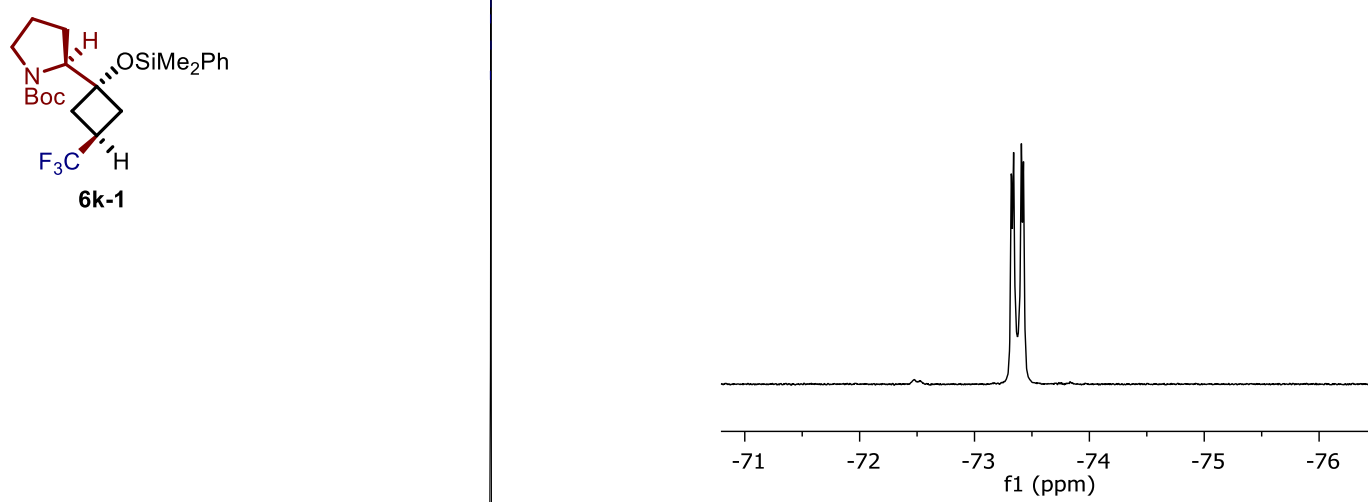

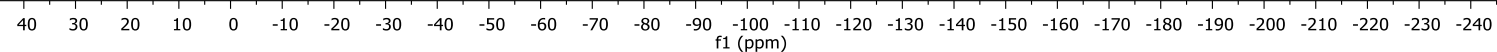


${ }^{1} \mathrm{H}$ NMR of compound $6 \mathbf{6}\left(500 \mathrm{MHz}, \mathrm{CDCl}_{3}\right)$

ms17109_MSP800p

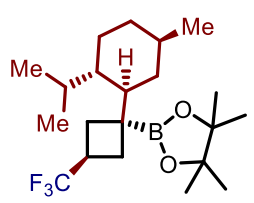

6I
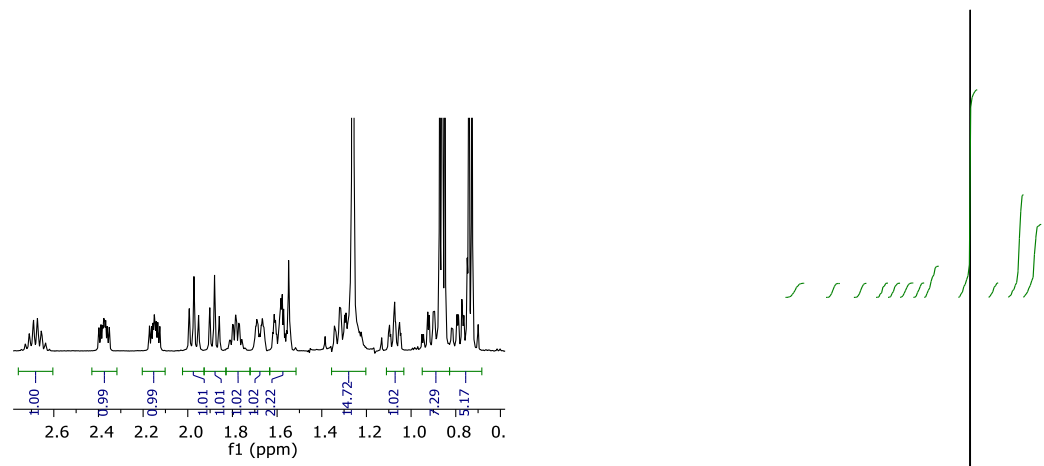

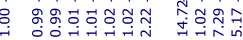

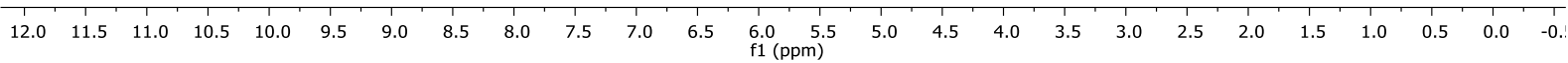

${ }^{13} \mathrm{C}$ NMR of compound $6 \mathbf{l}\left(125 \mathrm{MHz}, \mathrm{CDCl}_{3}\right)$

ms17109_MSP800

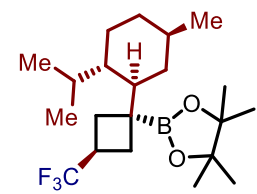

6I

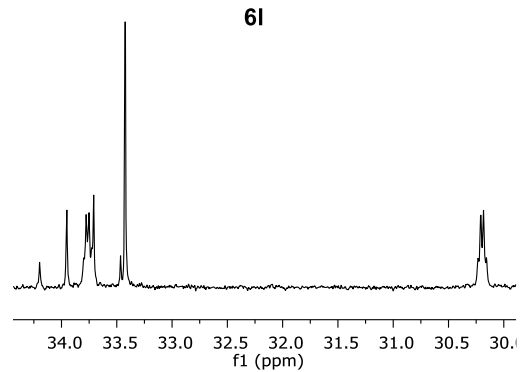

it

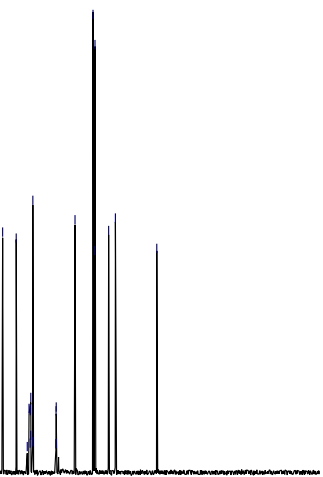

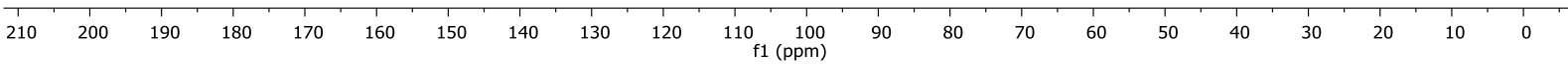


${ }^{1} \mathrm{~F}$ NMR of compound $6 \mathbf{1}\left(376 \mathrm{MHz}, \mathrm{CDCl}_{3}\right)$

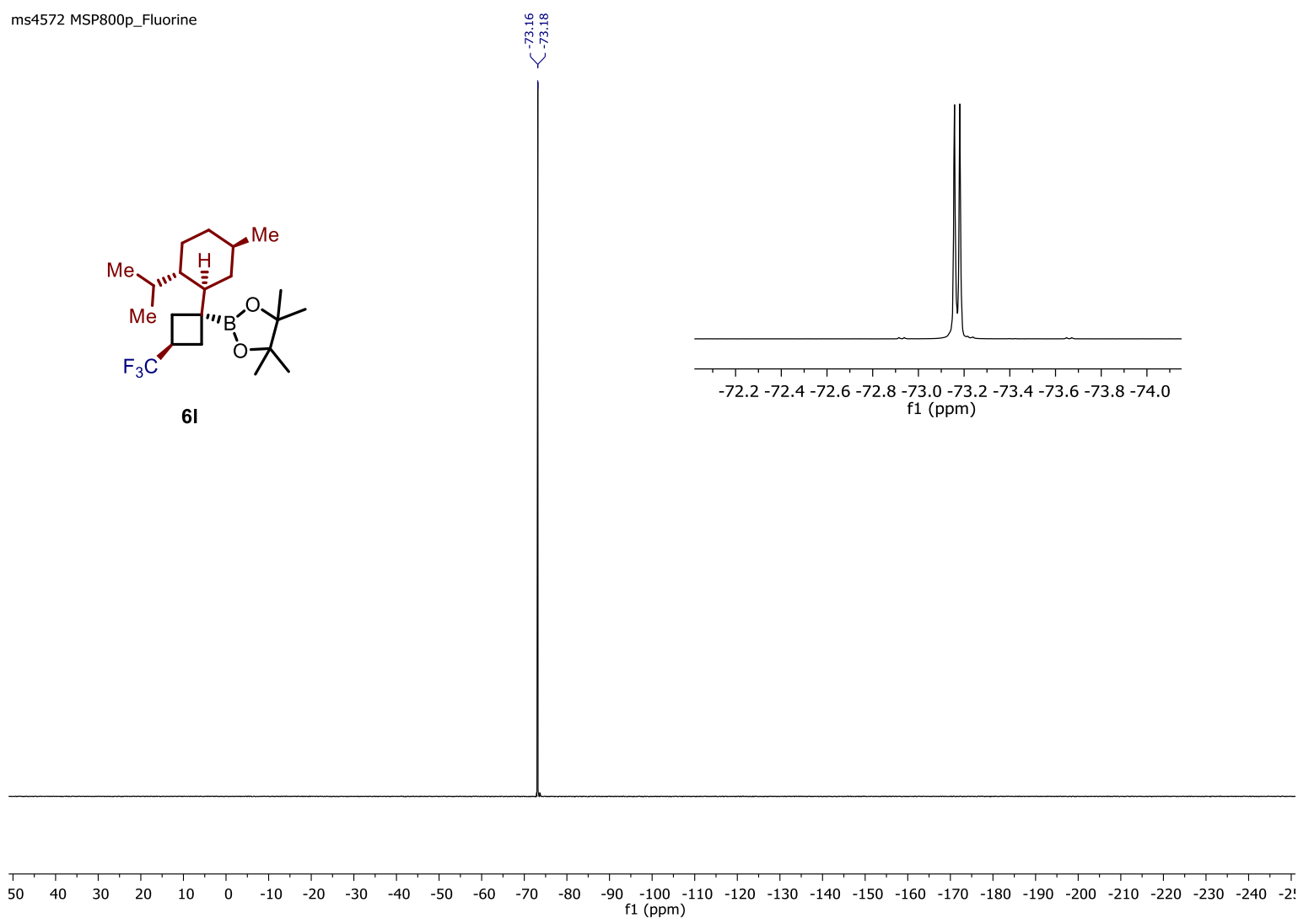


${ }^{13} \mathrm{C}$ NMR of compound $\mathbf{6 m}\left(100 \mathrm{MHz}, \mathrm{CDCl}_{3}\right)$

ms17408_MSP786bis_P_PROTONO1

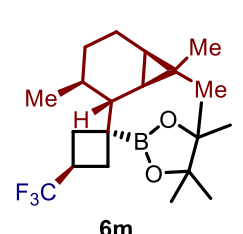

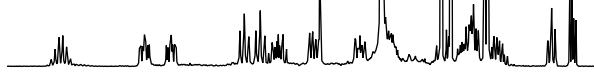

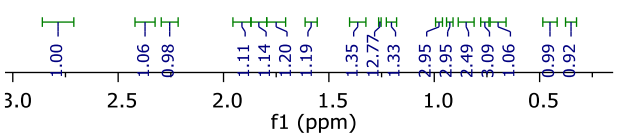

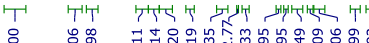

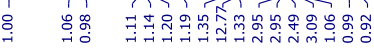

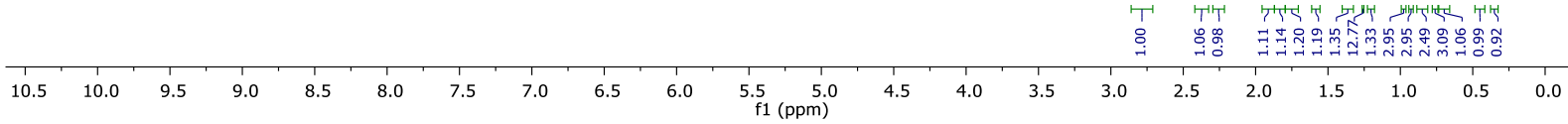

${ }^{13} \mathrm{C}$ NMR of compound $6 \mathbf{m}\left(125 \mathrm{MHz}, \mathrm{CDCl}_{3}\right)$

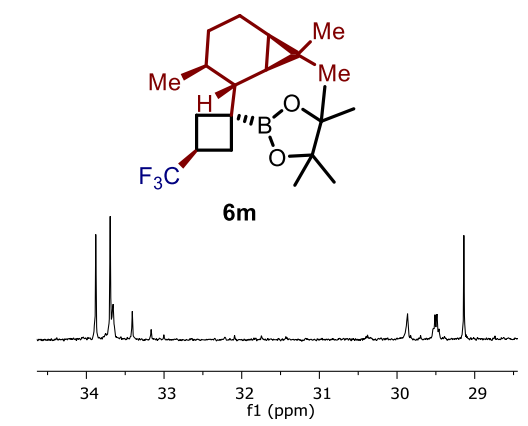

$6 m$

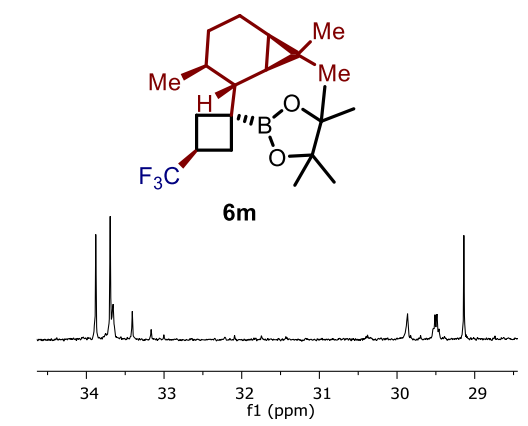

$\begin{array}{lllllllllll}210 & 200 & 190 & 180 & 170 & 160 & 150 & 140 & 130 & 120 & 110 \begin{array}{r}100 \\ \mathrm{f} 1(\mathrm{ppm})\end{array}\end{array}$

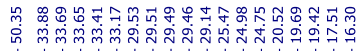

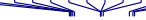
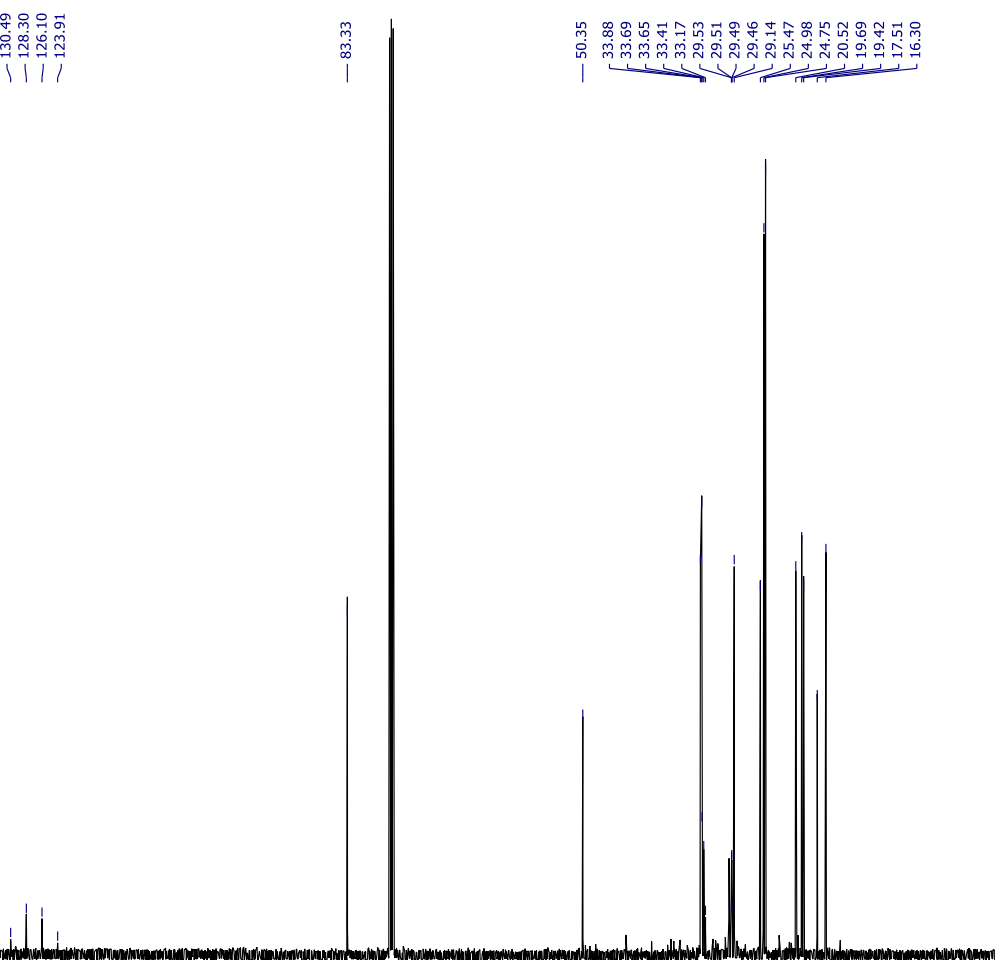
${ }^{19} \mathrm{~F}$ NMR of compound $\mathbf{6 m}\left(470 \mathrm{MHz}, \mathrm{CDCl}_{3}\right)$

ms27339_MSP786bis_P2_FLUORINE

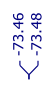

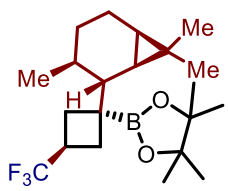

$6 \mathrm{~m}$

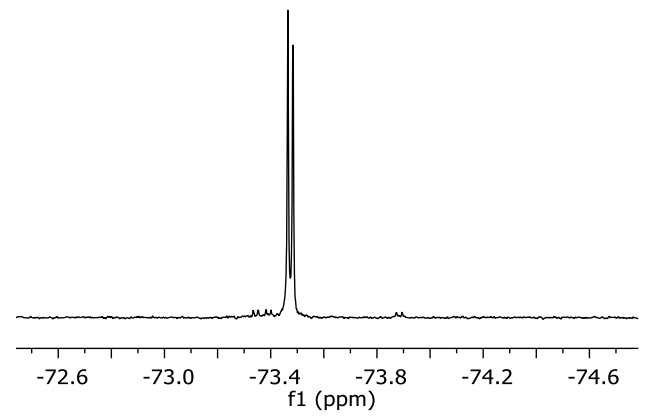

$\begin{array}{lllllllllllllllllllllllllllllll}40 & 30 & 20 & 10 & 0 & -10 & -20 & -30 & -40 & -50 & -60 & -70 & -80 & -90 & -100 & -110 & -120 & -130 & -140 & -150 & -160 & -170 & -180 & -190 & -200 & -210 & -220 & -230 & -240\end{array}$ 
${ }^{1} \mathrm{H}$ NMR of compound $\mathbf{6 n}\left(400 \mathrm{MHz}, \mathrm{CDCl}_{3}\right)$

va/ms4516 MSP799p
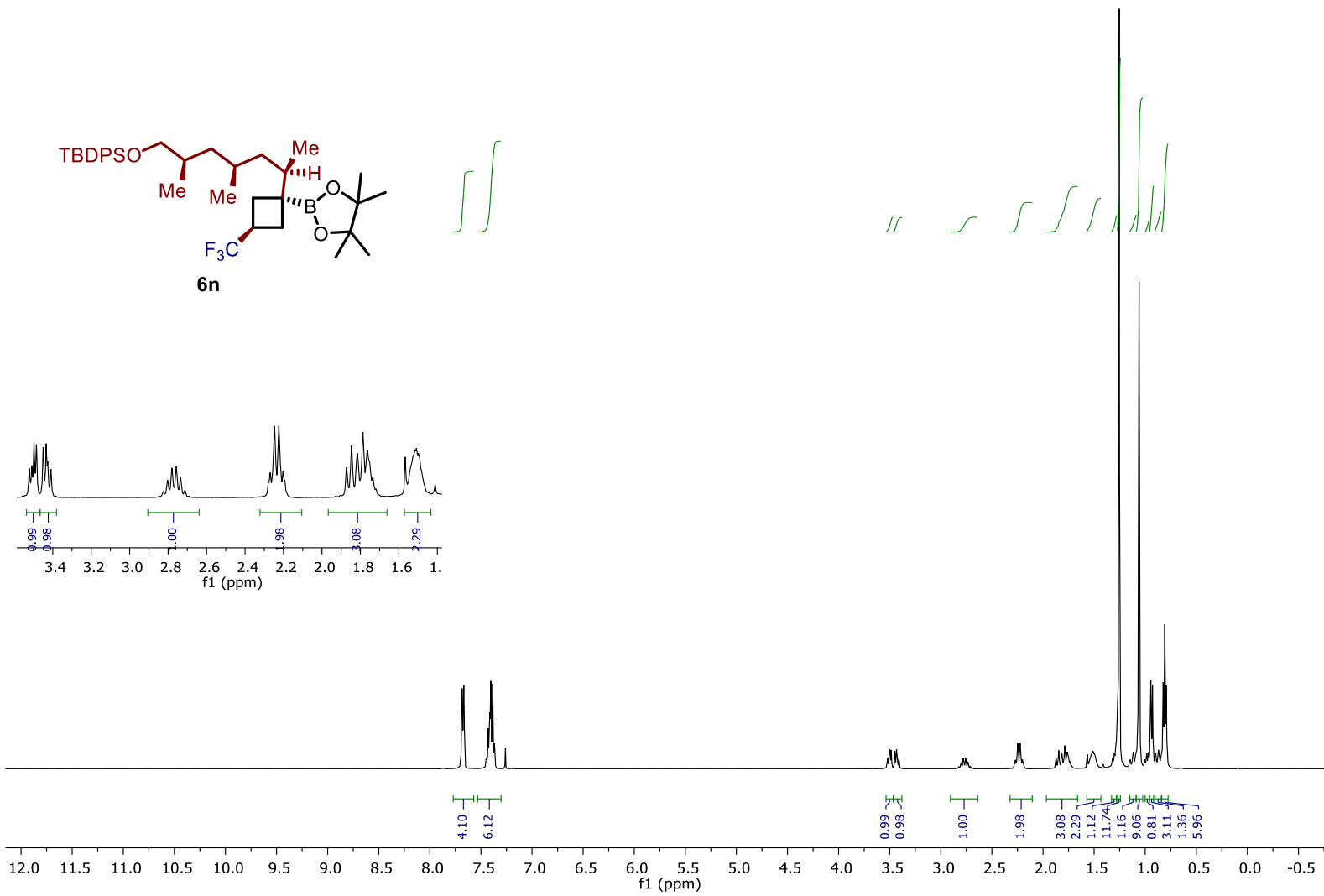

${ }^{13} \mathrm{C}$ NMR of compound $\mathbf{6 n}\left(125 \mathrm{MHz}, \mathrm{CDCl}_{3}\right)$

va/ms4516 MSP799p

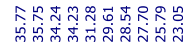

의

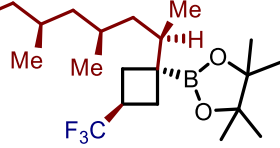

$6 n$
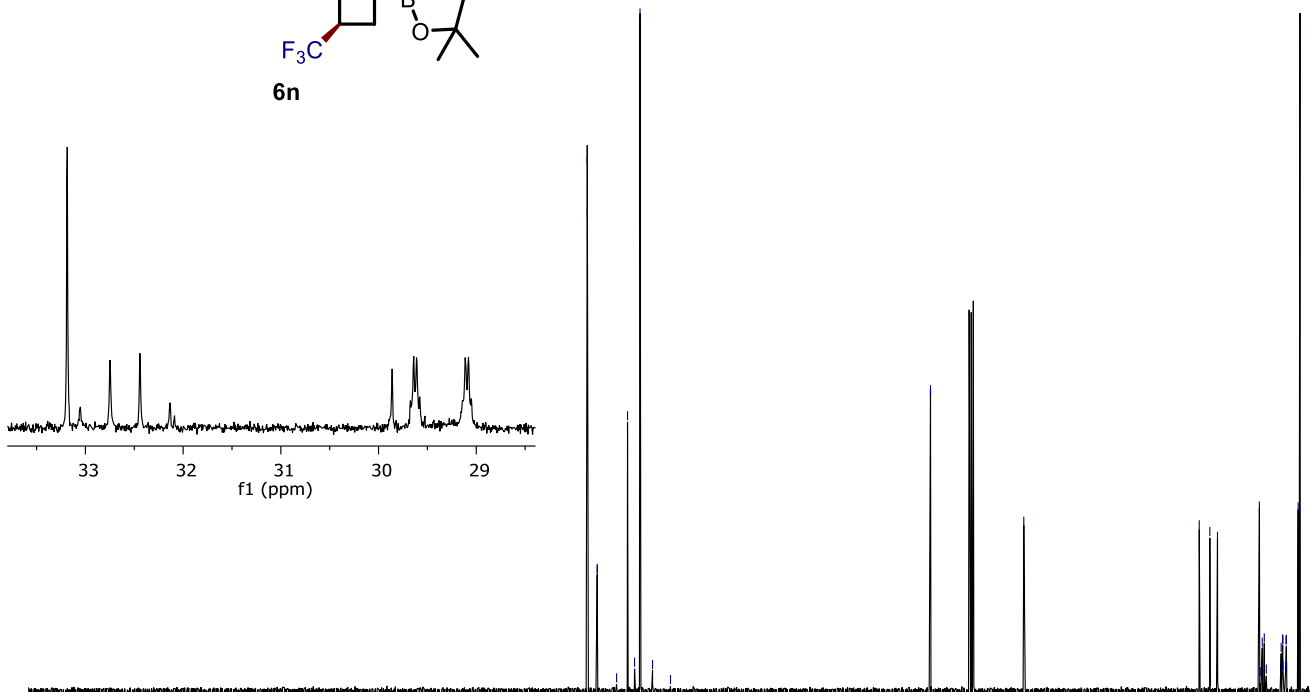

$\begin{array}{lll}210 & 200 \quad 190\end{array}$

$\begin{array}{llllllll}180 & 170 & 160 & 150 & 140 & 130 & 120 & \begin{array}{c}110 \\ \mathrm{f} 1(\mathrm{ppm})\end{array}\end{array}$ 
${ }^{19} \mathrm{~F}$ NMR of compound $6 \mathbf{n}\left(376 \mathrm{MHz}, \mathrm{CDCl}_{3}\right)$

va/ms4516 MSP799p

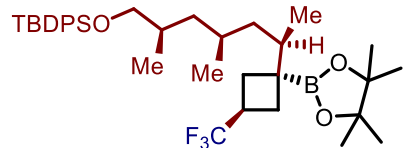

6n

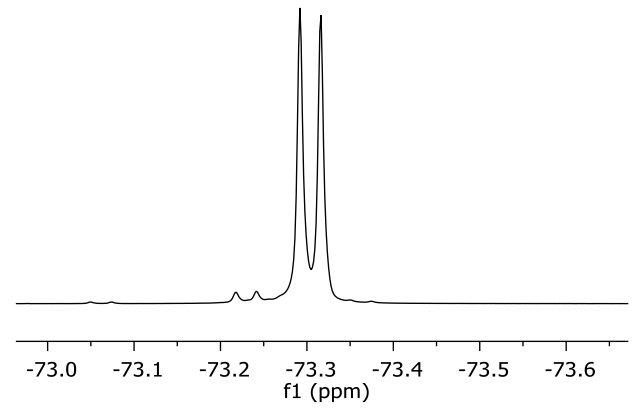

조ำำ

\&

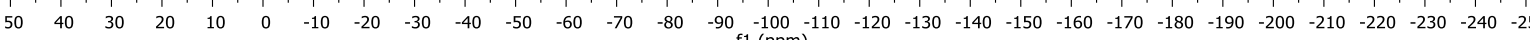


${ }^{1} \mathrm{H}$ NMR of compound $60\left(500 \mathrm{MHz}, \mathrm{CDCl}_{3}\right)$

ms17349_MSP784P_PROTON01
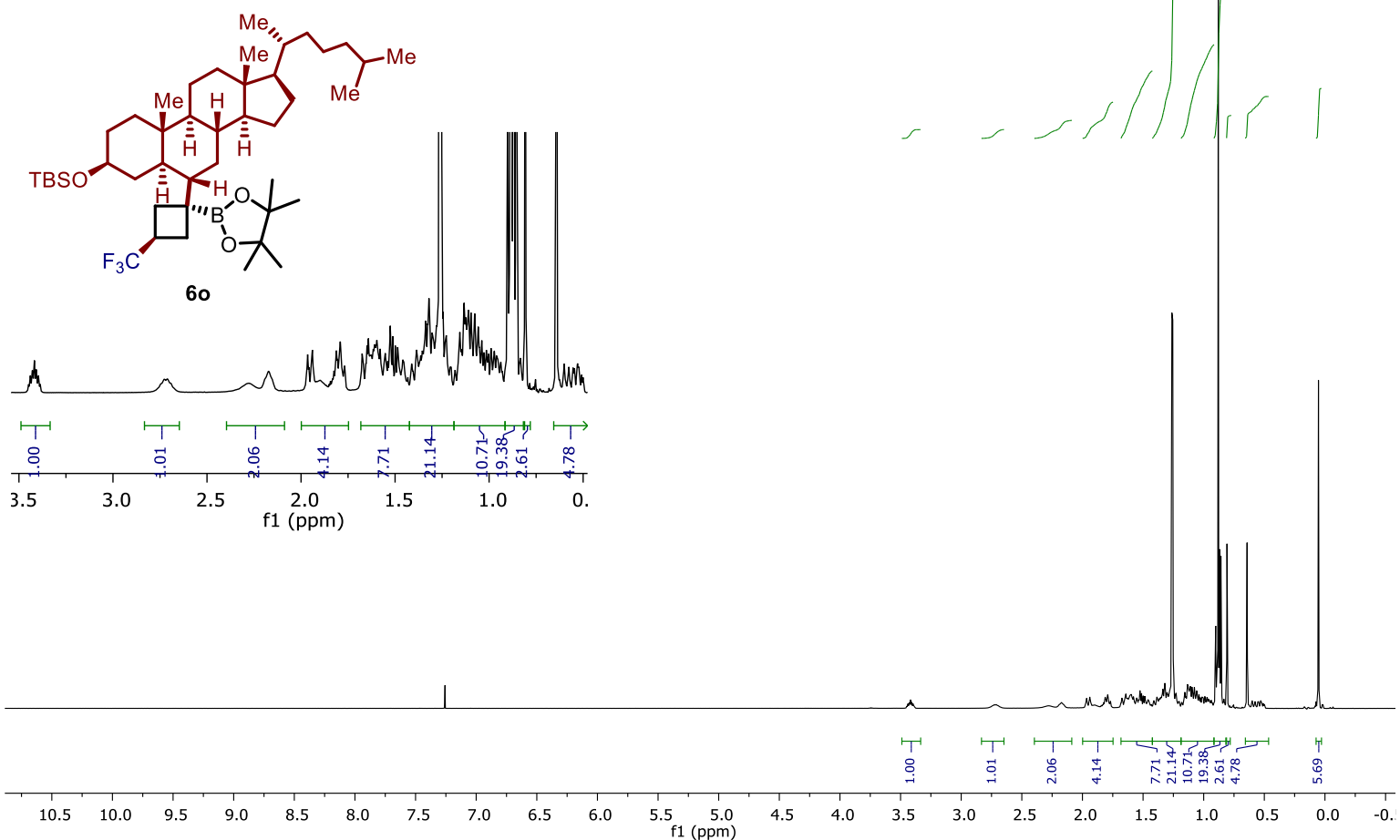

${ }^{13} \mathrm{C}$ NMR of compound $60\left(125 \mathrm{MHz}, \mathrm{CDCl}_{3}\right)$

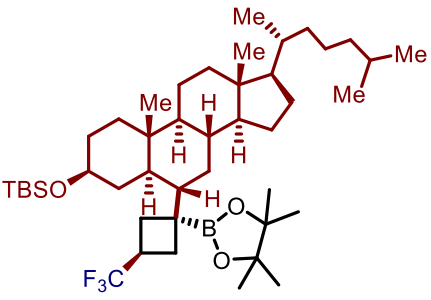

60

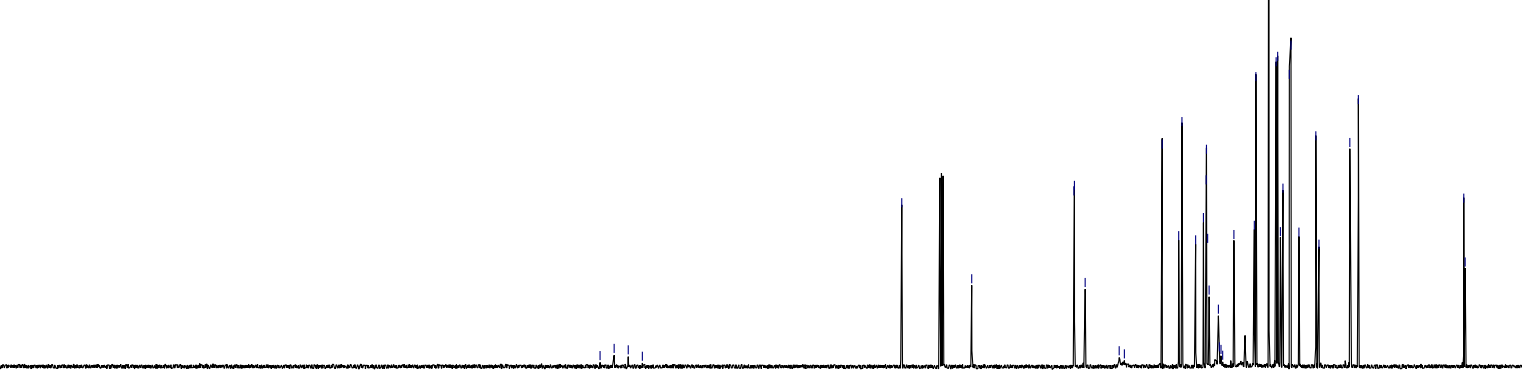

$\begin{array}{lllllllllllllllllllllllll}220 & 210 & 200 & 190 & 180 & 170 & 160 & 150 & 140 & 130 & 120 & 110 & 100 & 90 & 80 & 70 & 60 & 50 & 40 & 30 & 20 & 10 & 0 & -10\end{array}$ 
${ }^{1} \mathrm{H}$ NMR of compound $\mathbf{6 o}\left(400 \mathrm{MHz}, \mathrm{CDCl}_{3}\right)$

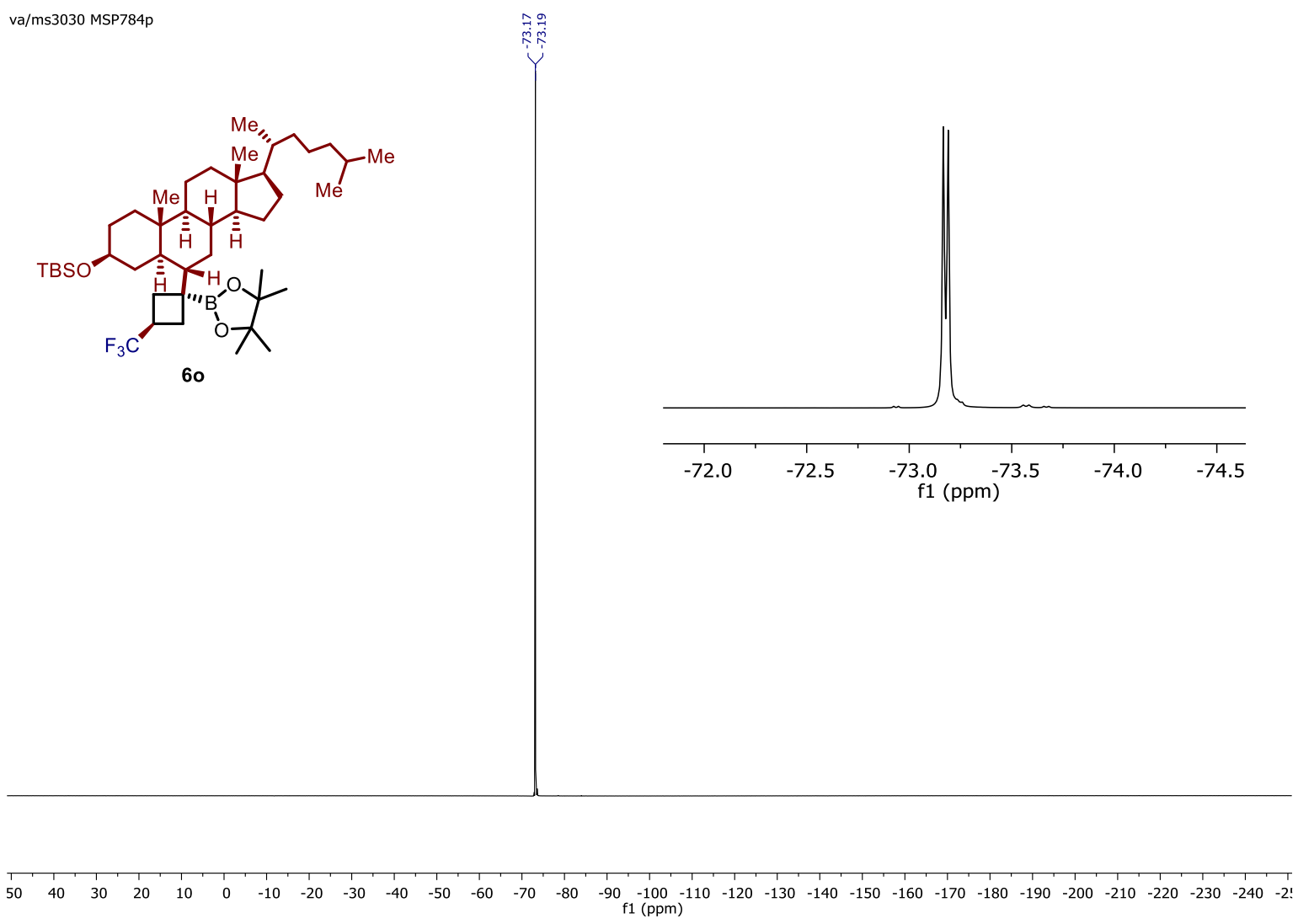


${ }^{1} \mathrm{H}$ NMR of compound $\mathbf{6 p}\left(500 \mathrm{MHz}, \mathrm{CDCl}_{3}\right)$

ms26319_MSP686p_PROTON_001

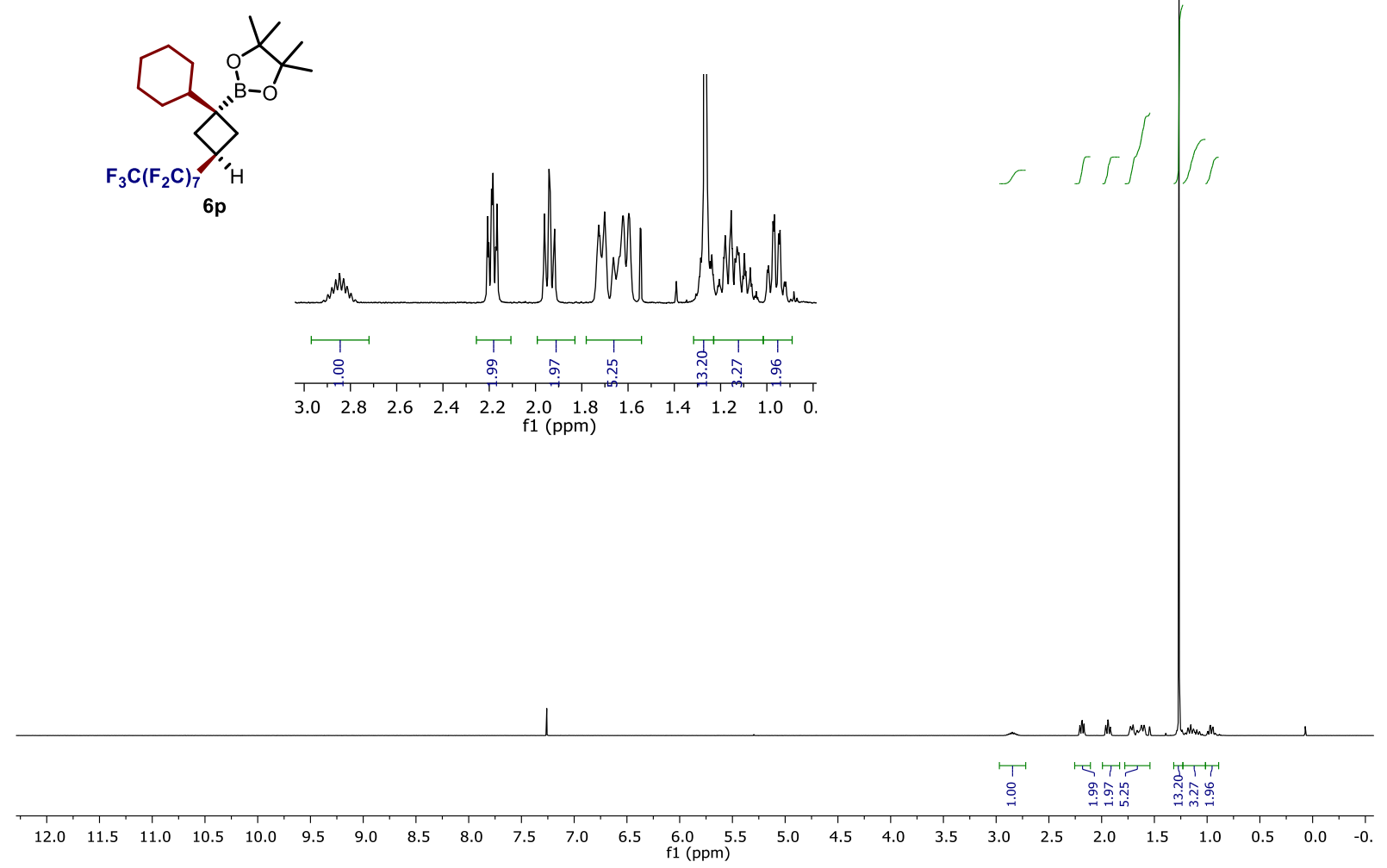

${ }^{13} \mathrm{C} \mathrm{NMR}$ of compound $\mathbf{6 p}\left(125 \mathrm{MHz}, \mathrm{CDCl}_{3}\right)$

ms26319_MSP686p_CARBON_001
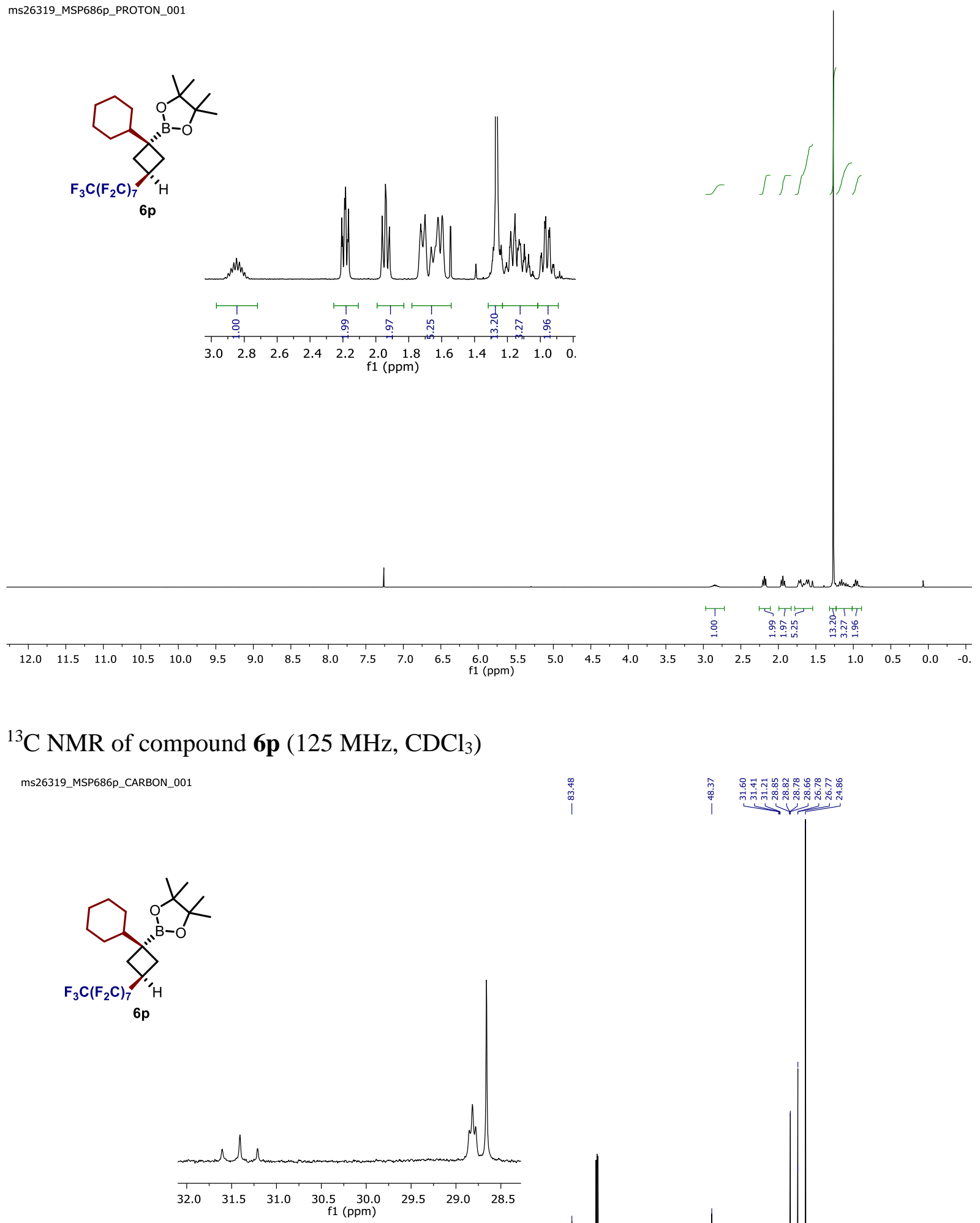
${ }^{19} \mathrm{~F}$ NMR of compound $\mathbf{6 p}\left(470 \mathrm{MHz}, \mathrm{CDCl}_{3}\right)$

ms26319_MSP686p_FLUORINE_00 STANDARD PROTON PARAMETERS

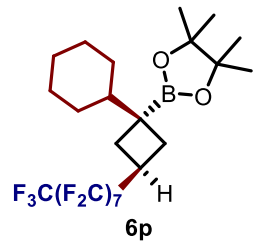
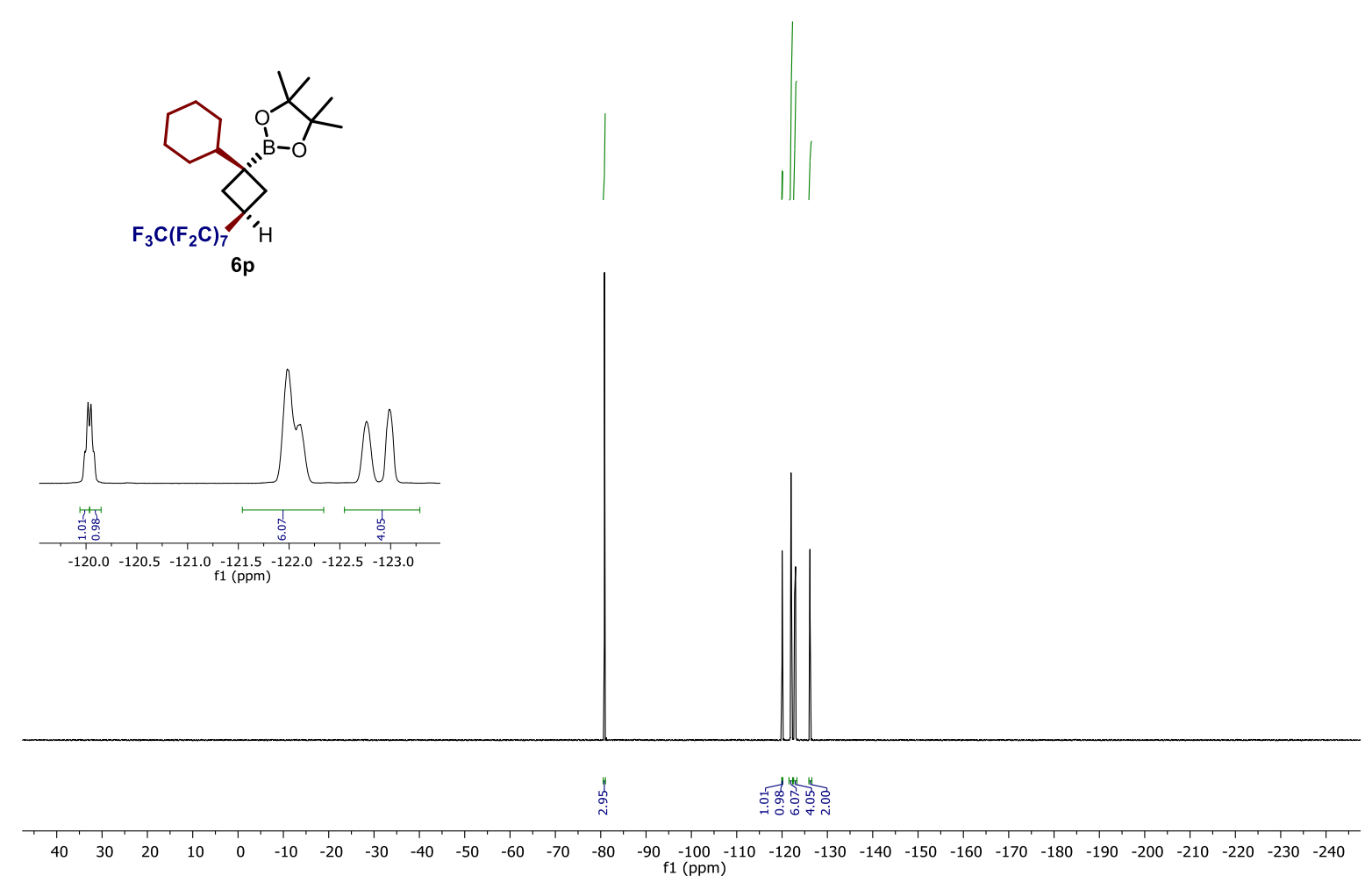
${ }^{1} \mathrm{H}$ NMR of compound $\mathbf{6 q}\left(500 \mathrm{MHz}, \mathrm{CDCl}_{3}\right)$

9884 MSP767_p2.10.fid
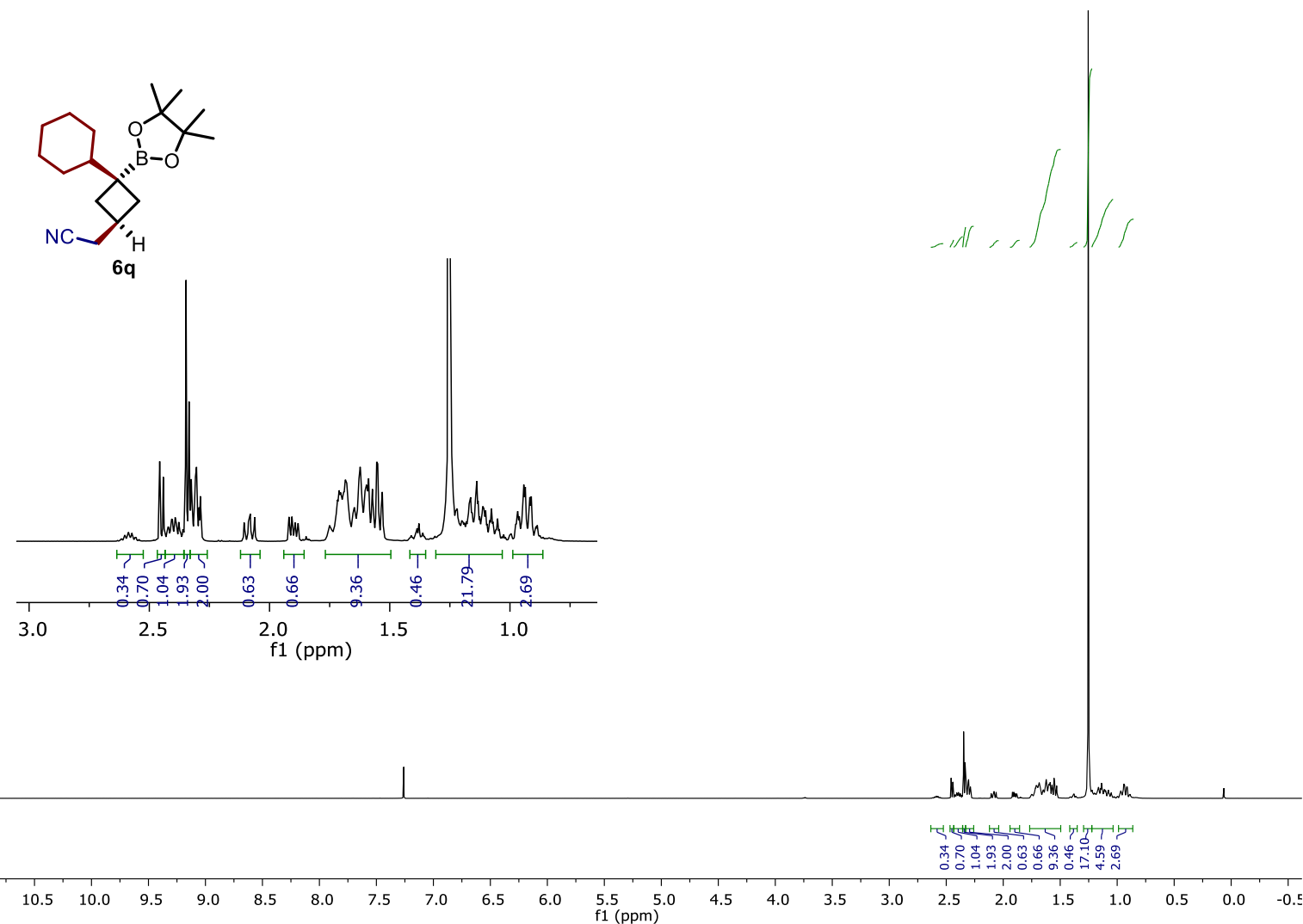

${ }^{13} \mathrm{C}$ NMR of compound $\mathbf{6 q}\left(125 \mathrm{MHz}, \mathrm{CDCl}_{3}\right)$

9884 MSP767_p2.11.fid

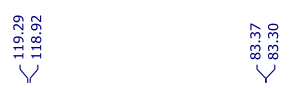

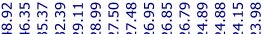

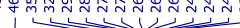

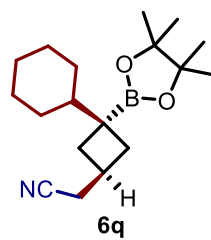


${ }^{1} \mathrm{H}$ NMR of compound $6 \mathbf{r}\left(500 \mathrm{MHz}, \mathrm{CDCl}_{3}\right)$

ms27116_MSP768p_PROTON_001
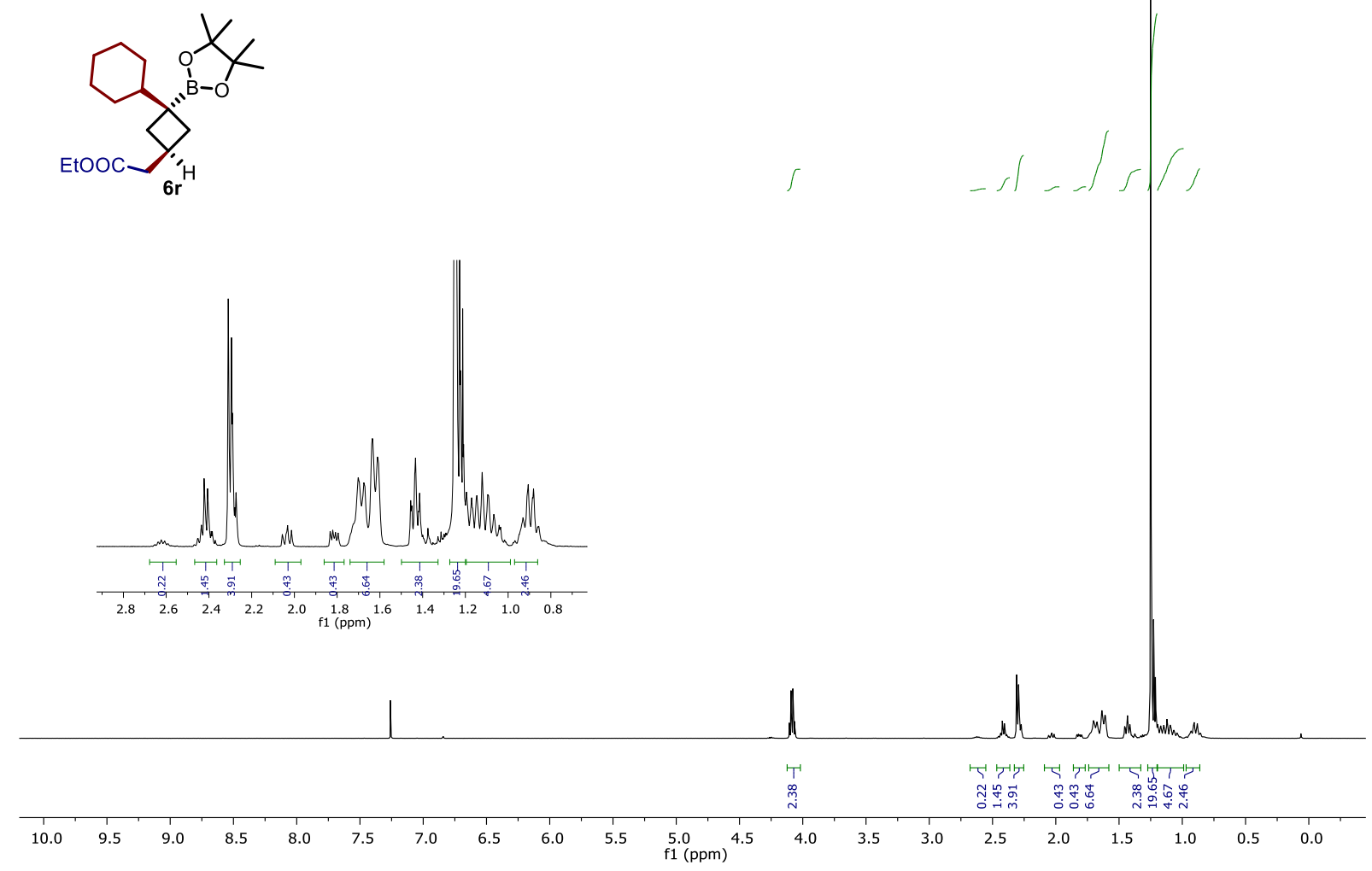

${ }^{13} \mathrm{C}$ NMR of compound $6 \mathbf{r}\left(125 \mathrm{MHz}, \mathrm{CDCl}_{3}\right)$

ms27116_MSP768p_CARBON_001 울
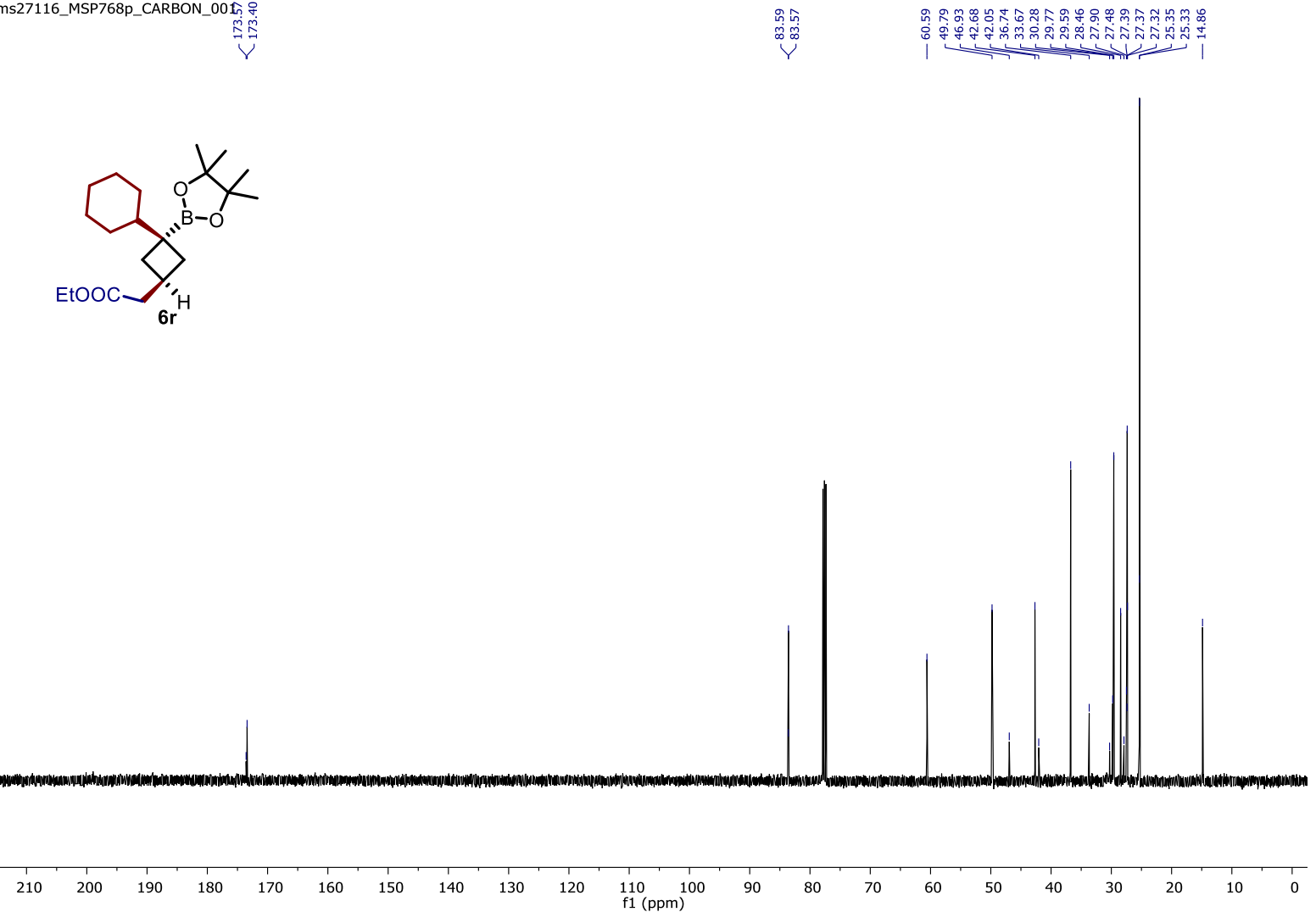
${ }^{1} \mathrm{H}$ NMR of compound $\mathbf{6 s}\left(400 \mathrm{MHz}, \mathrm{CDCl}_{3}\right)$

va/ms1803 MSP778p

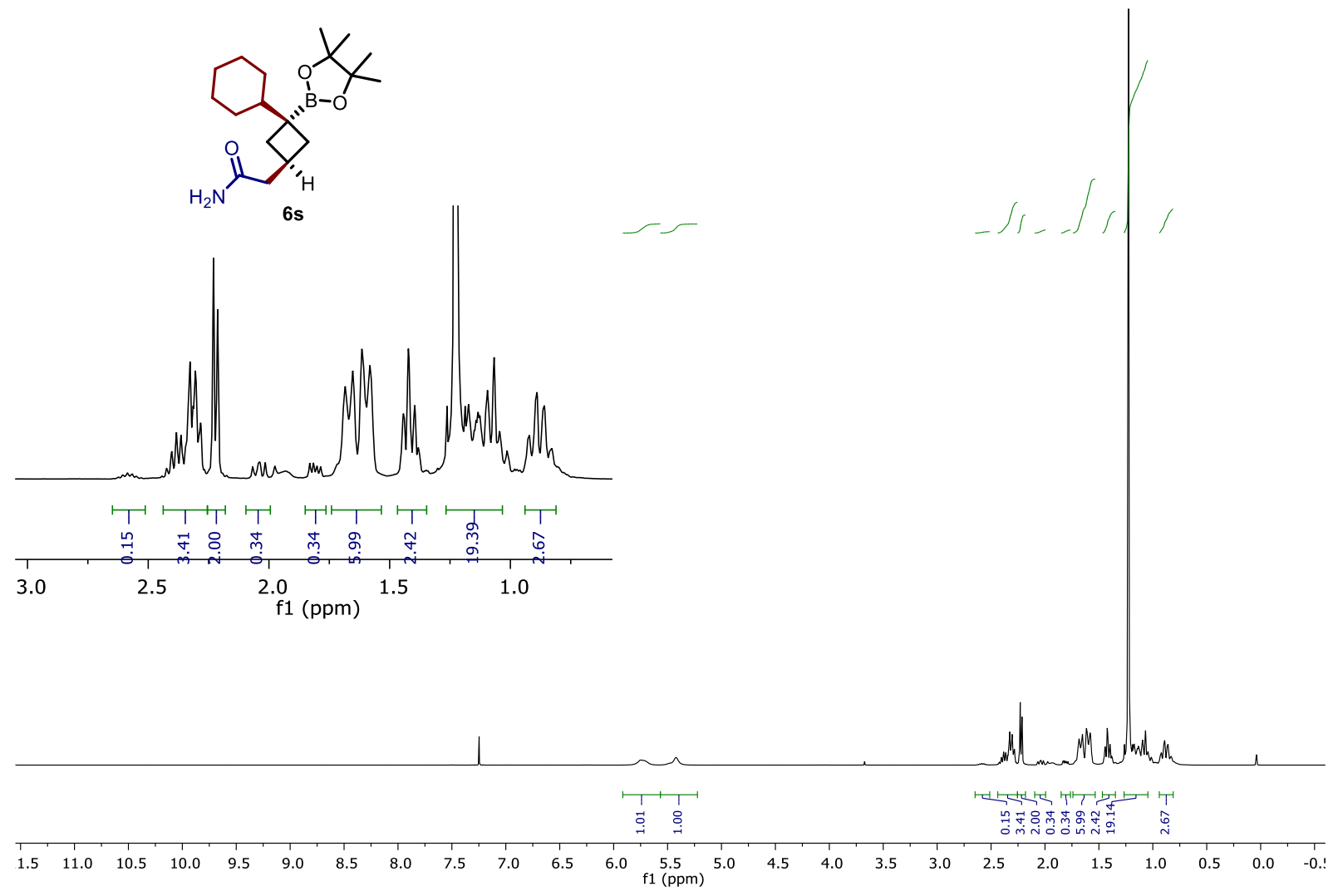

${ }^{13} \mathrm{C}$ NMR of compound $\mathbf{6 s}\left(100 \mathrm{MHz}, \mathrm{CDCl}_{3}\right)$

va/ms1803 MSP778p
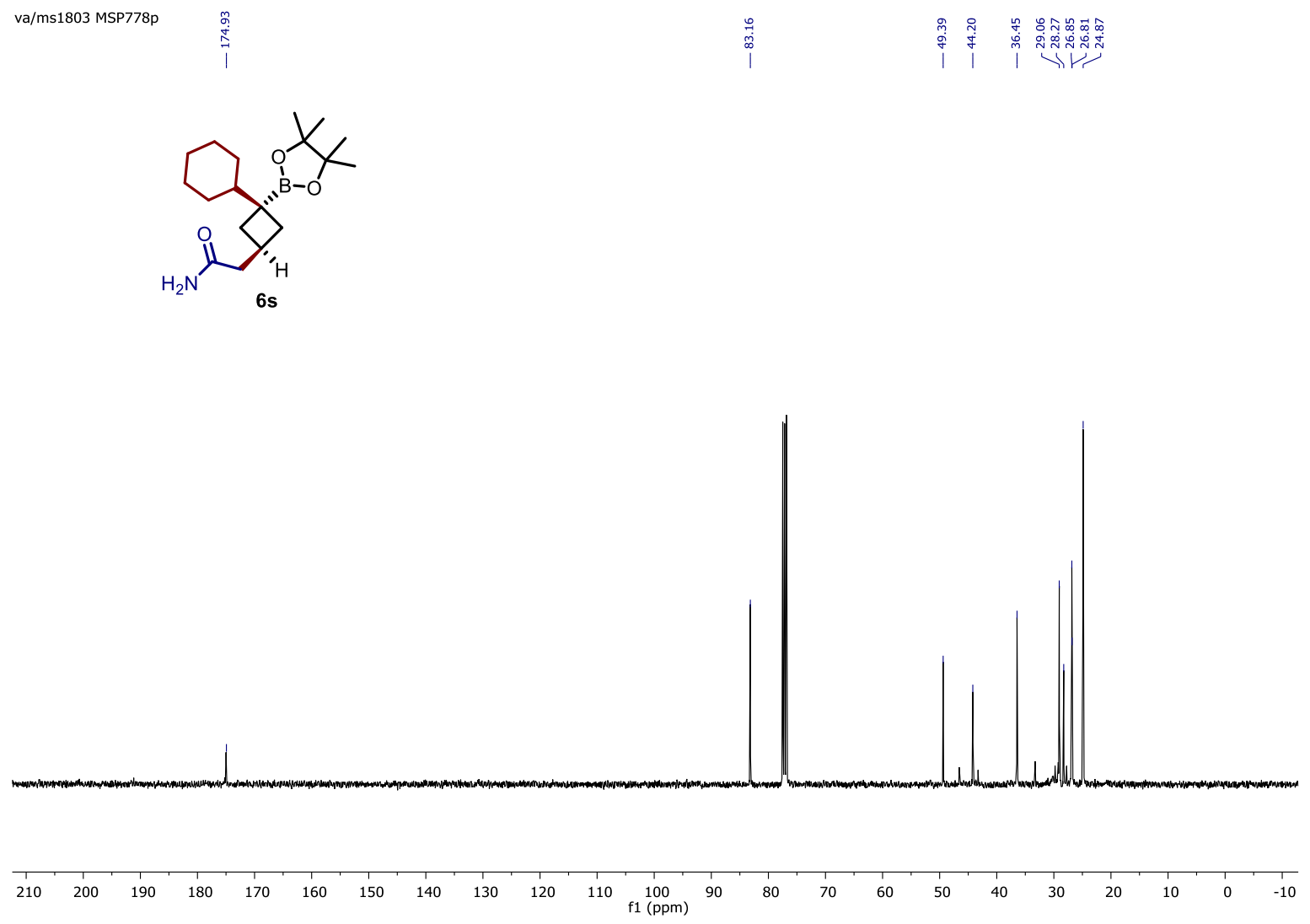

S80 
${ }^{1} \mathrm{H}$ NMR of compound $\mathbf{6 t}\left(400 \mathrm{MHz}, \mathrm{CDCl}_{3}\right)$

ms48029_MSP774p_PROTON_01

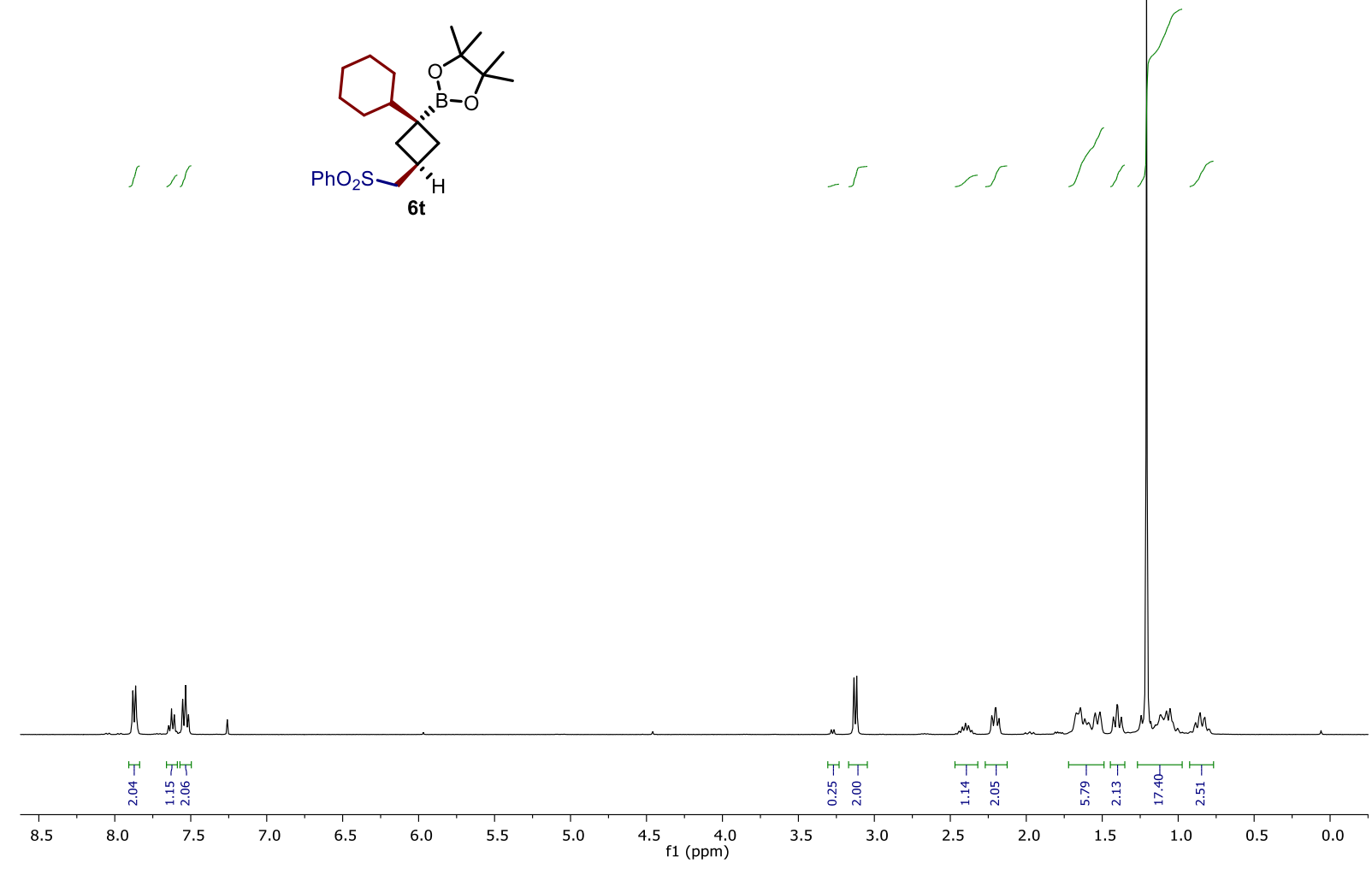

${ }^{13} \mathrm{C}$ NMR of compound $6 \mathbf{t}\left(100 \mathrm{MHz}, \mathrm{CDCl}_{3}\right)$

ms48029_MSP774p_CARBON_01

0
0
0

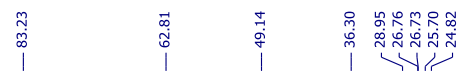

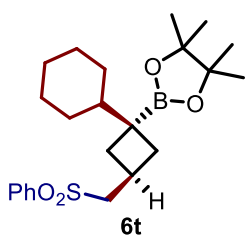

$\begin{array}{lllllllllll}210 & 200 & 190 & 180 & 170 & 160 & 150 & 140 & 130 & 120 & 110 \\ \mathrm{f} 1(\mathrm{ppm}) & 100\end{array}$ 
${ }^{1} \mathrm{H}$ NMR of compound $\mathbf{6 w}\left(500 \mathrm{MHz}, \mathrm{CDCl}_{3}\right)$

ms17196_MSP820p_PROTONO1
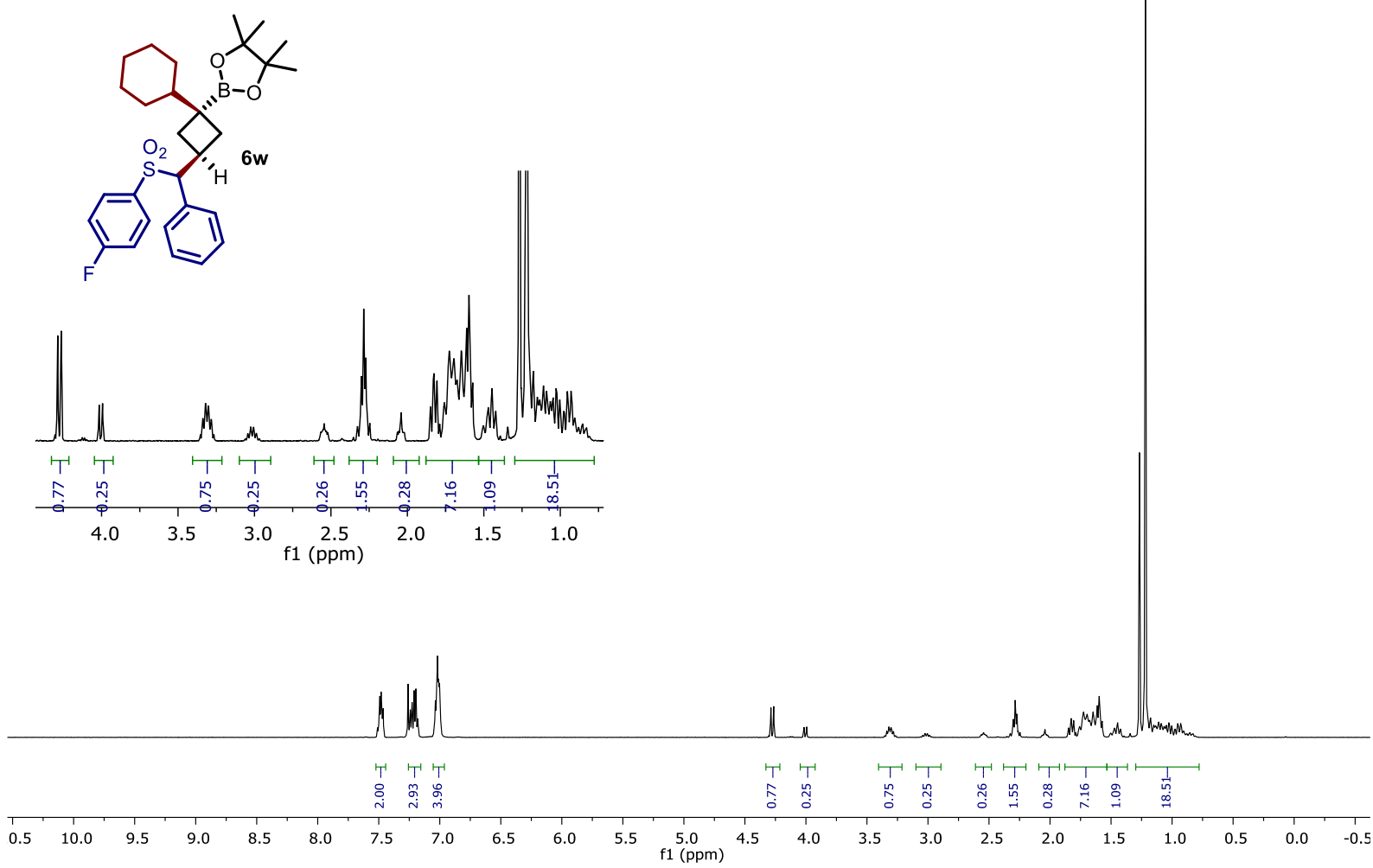

${ }^{13} \mathrm{C}$ NMR of compound $\mathbf{6 w}\left(125 \mathrm{MHz}, \mathrm{CDCl}_{3}\right)$

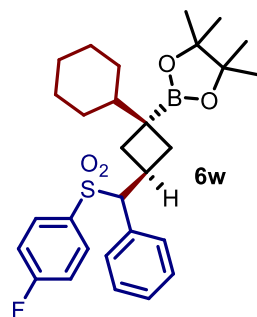


${ }^{1} \mathrm{H}$ NMR of compound $\mathbf{6 u}\left(400 \mathrm{MHz}, \mathrm{CDCl}_{3}\right)$

42968 MSP816p
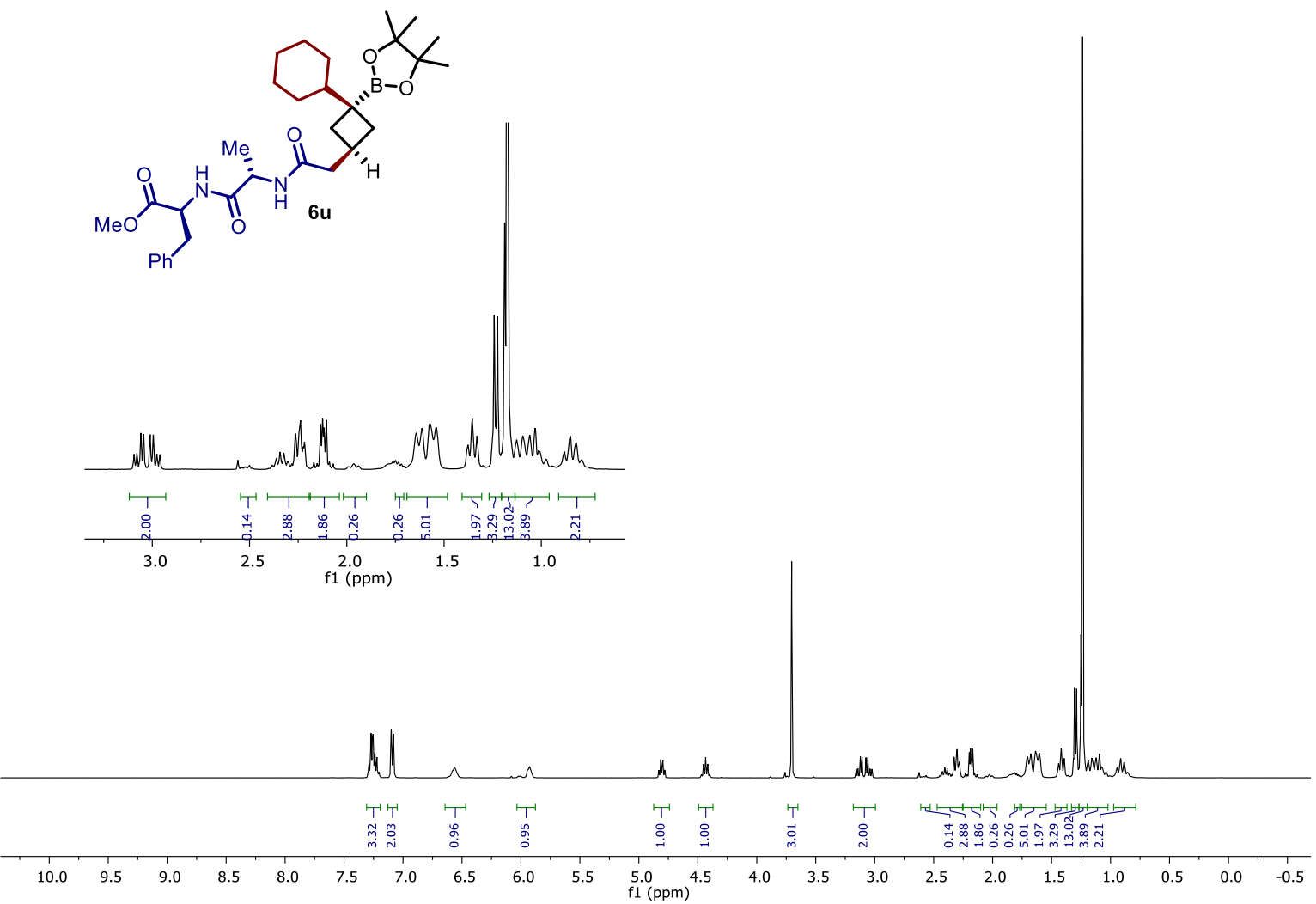

${ }^{13} \mathrm{C}$ NMR of compound $6 \mathbf{u}\left(100 \mathrm{MHz}, \mathrm{CDCl}_{3}\right)$
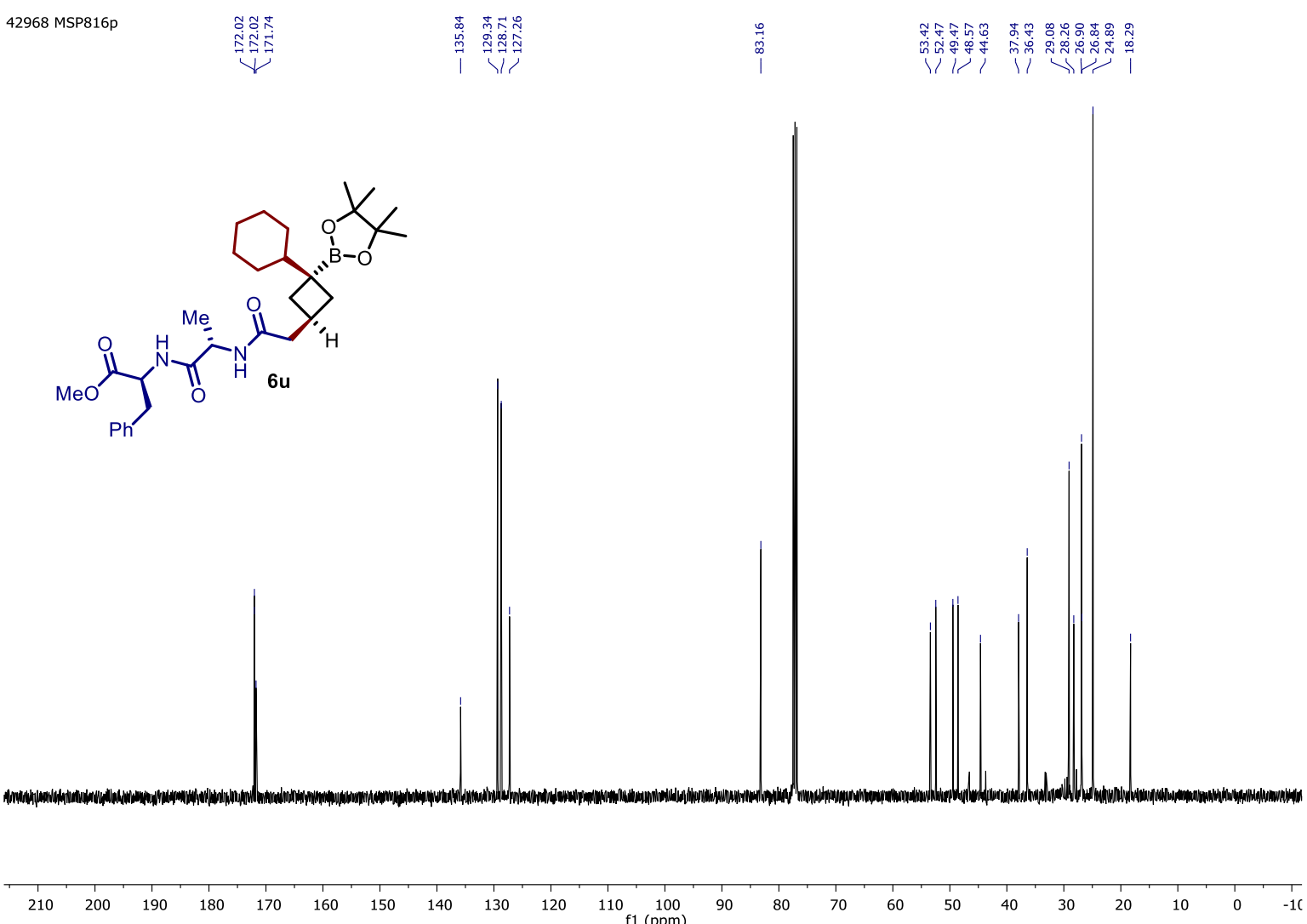
${ }^{1} \mathrm{H}$ NMR of compound $\mathbf{6 v}\left(500 \mathrm{MHz}, \mathrm{CDCl}_{3}\right)$

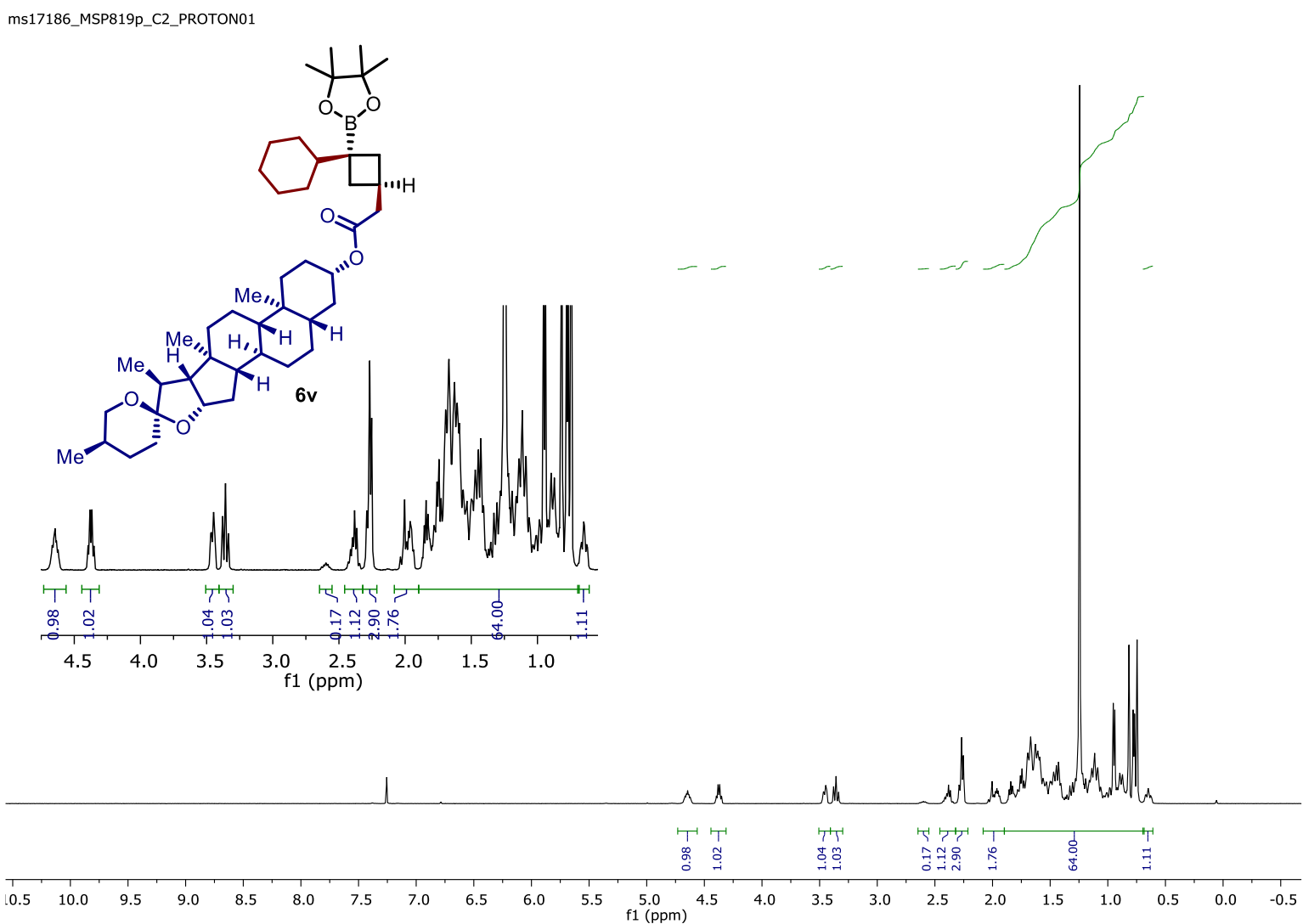

${ }^{13} \mathrm{C}$ NMR of compound 6v (125 MHz, $\left.\mathrm{CDCl}_{3}\right)$

ms17186_MSP819p_C2_CARBONOQ

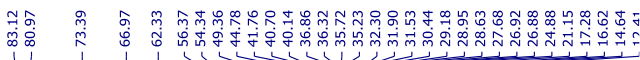

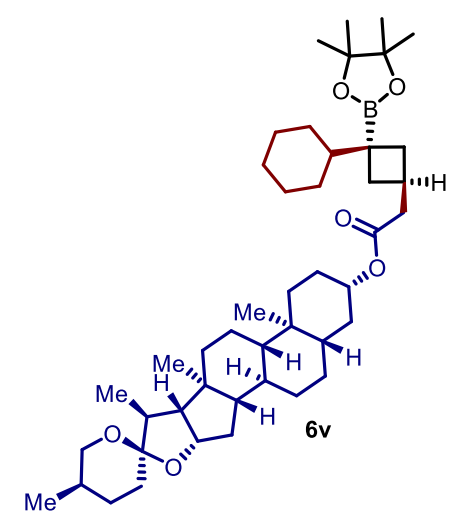

$\begin{array}{llllllllllll}210 & 200 & 190 & 180 & 170 & 160 & 150 & 140 & 130 & 120 & 110 & 100\end{array}$ 
${ }^{1} \mathrm{H}$ NMR of compound 9a (400 MHz, $\mathrm{CDCl}_{3}$ )

va/ms83845 MSP811p

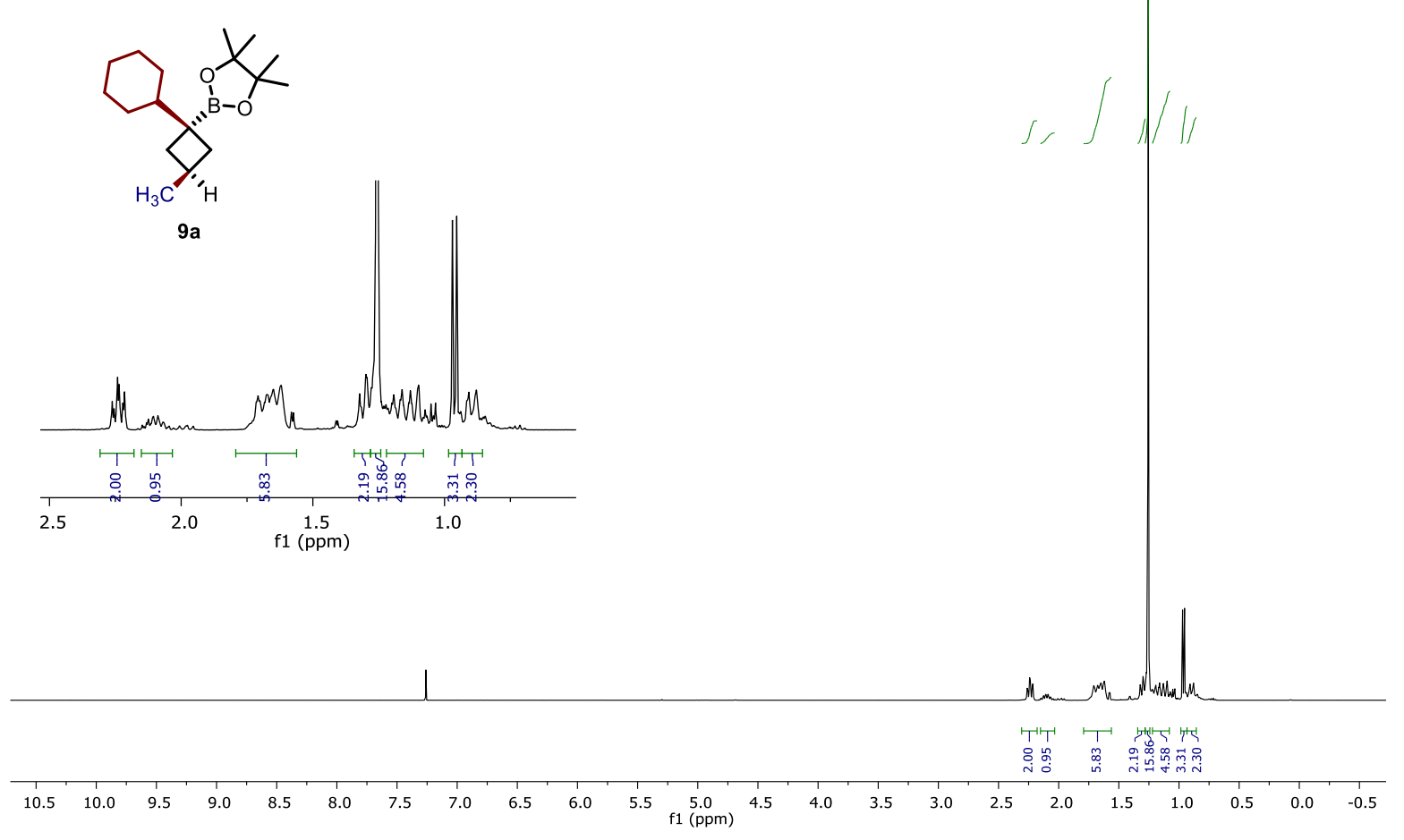

${ }^{13} \mathrm{C}$ NMR of compound $9 \mathbf{a}\left(100 \mathrm{MHz}, \mathrm{CDCl}_{3}\right)$

va/ms83845 MSP811p
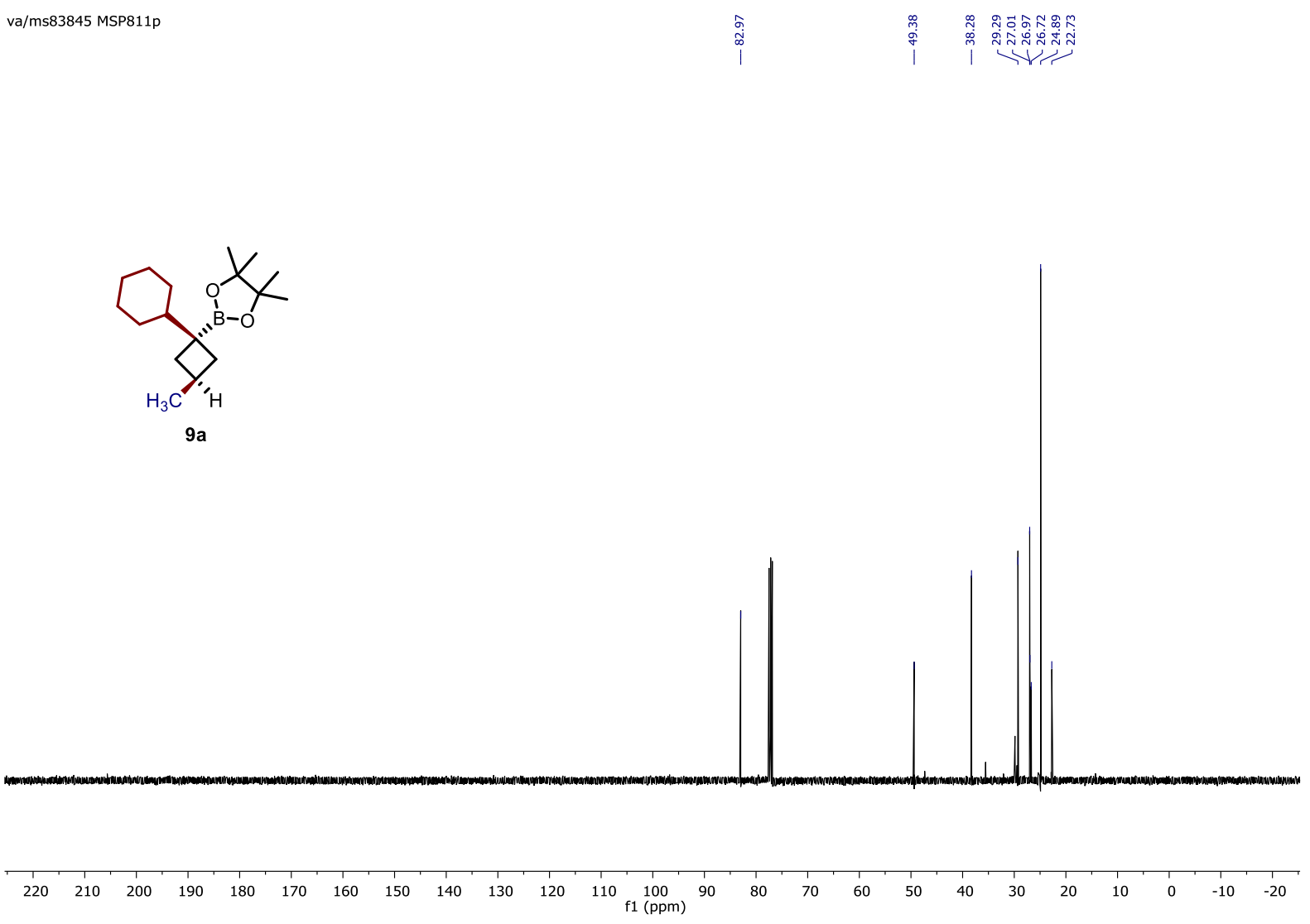

S85 
${ }^{1} \mathrm{H} \mathrm{NMR}$ of compound $9 \mathbf{b}\left(400 \mathrm{MHz}, \mathrm{CDCl}_{3}\right)$

va/ms1770 MSP825p2

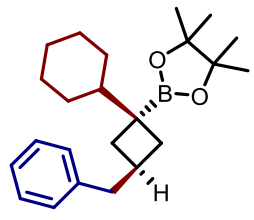

$9 b$

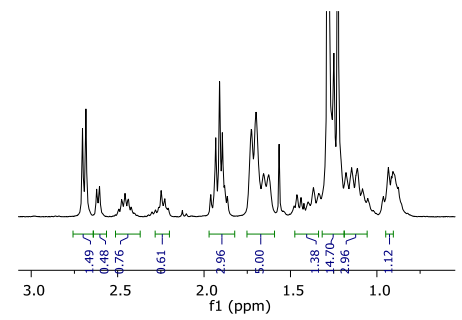

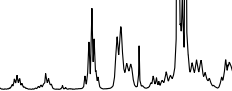

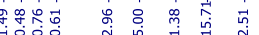

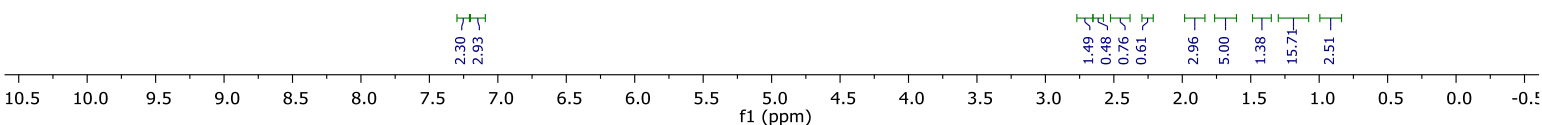

${ }^{13} \mathrm{C}$ NMR of compound $9 \mathrm{~b}\left(100 \mathrm{MHz}, \mathrm{CDCl}_{3}\right)$

$\mathrm{va} / \mathrm{ms} 1770 \mathrm{MSP} 825 \mathrm{p} 2$

封

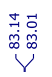

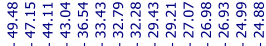

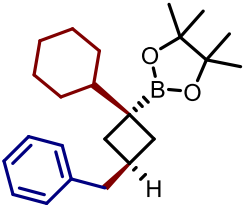

$9 b$

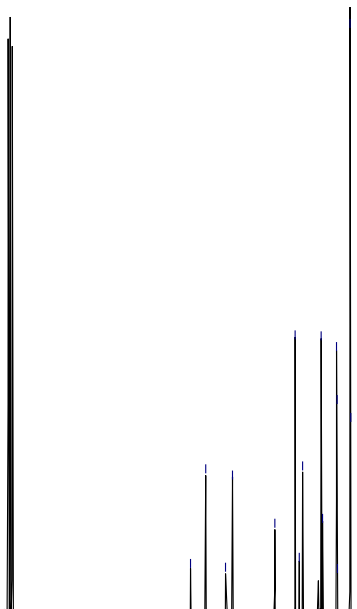

$\begin{array}{llllllllllll}220 & 210 & 200 & 190 & 180 & 170 & 160 & 150 & 140 & 130 & 120 & \begin{array}{l}110 \\ \mathrm{f} 1(\mathrm{ppm})\end{array}\end{array}$ 
${ }^{1} \mathrm{H} \mathrm{NMR}$ of compound $\mathbf{1 0 a}\left(500 \mathrm{MHz}, \mathrm{CDCl}_{3}\right)$

ms17464_MSP845P_PROTON01

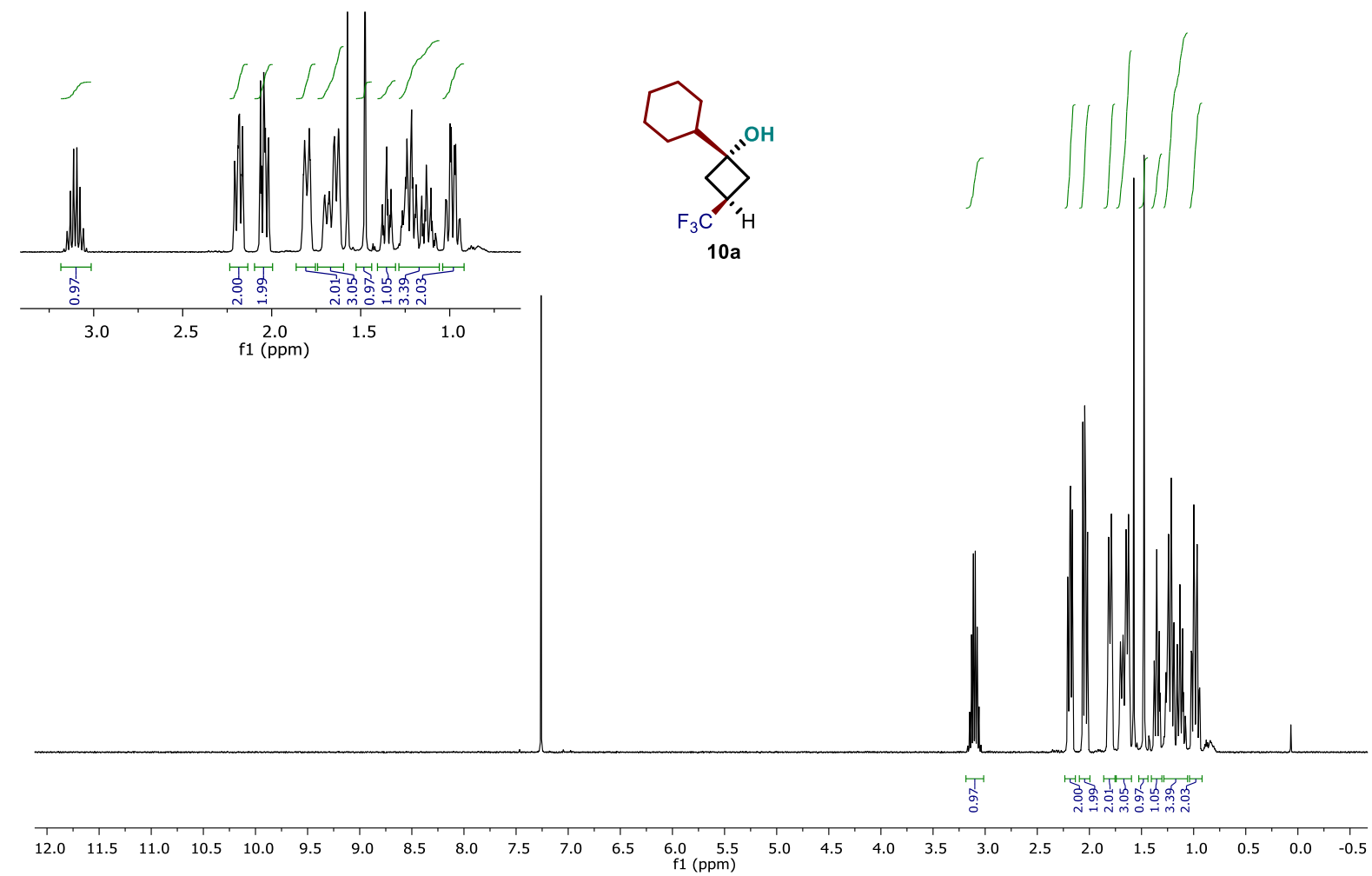

${ }^{13} \mathrm{C} \mathrm{NMR}$ of compound $\mathbf{1 0 a}\left(125 \mathrm{MHz}, \mathrm{CDCl}_{3}\right)$

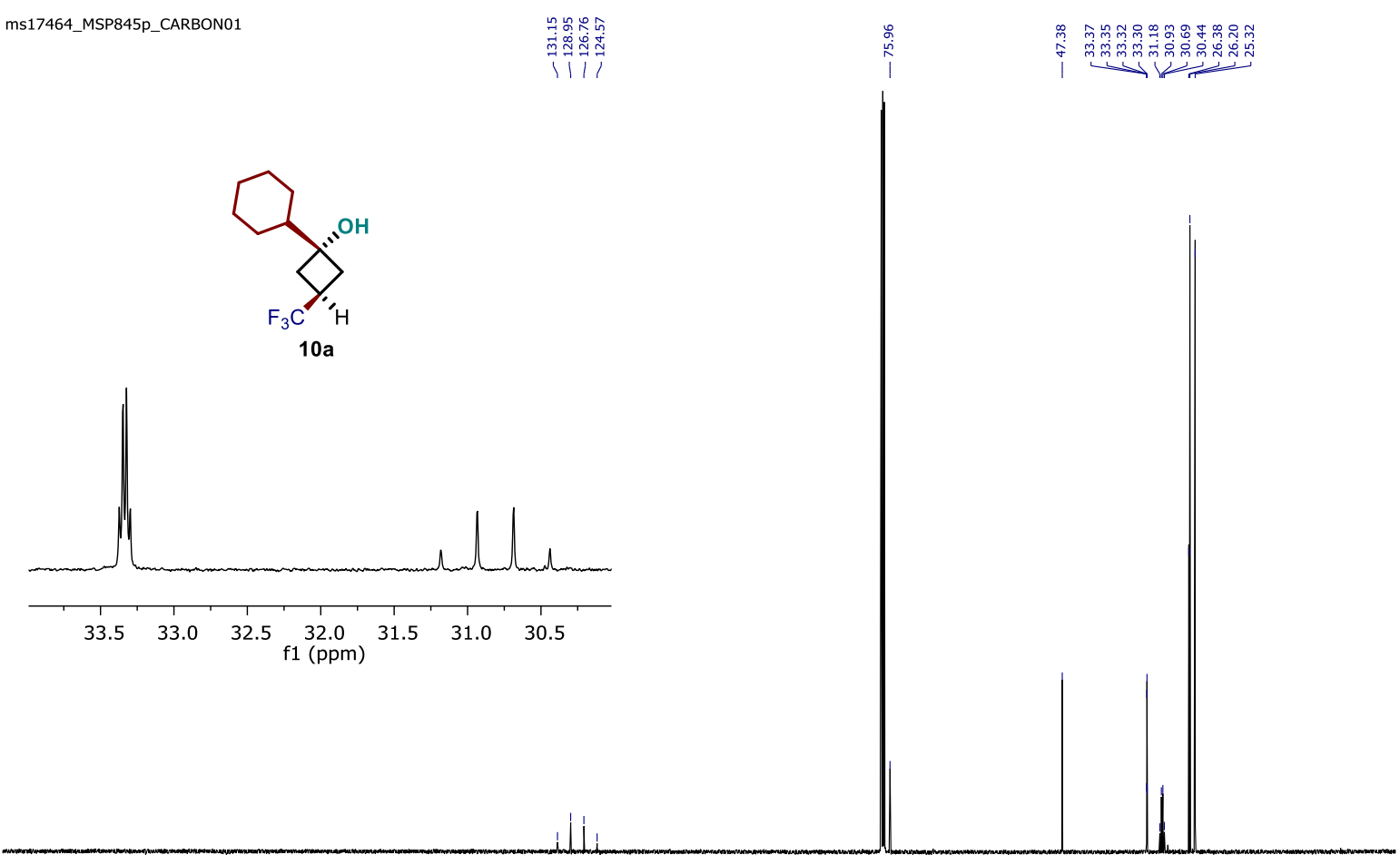

$\begin{array}{lllllllllllllllllllllllllll} & 220 & 210 & 200 & 190 & 180 & 170 & 160 & 150 & 140 & 130 & 120 & 110 & 100 & 90 & 80 & 70 & 60 & 50 & 40 & 30 & 20 & 10 & 0\end{array}$ 
${ }^{19} \mathrm{~F}$ NMR of compound $\mathbf{1 0 a}\left(376 \mathrm{MHz}, \mathrm{CDCl}_{3}\right)$

$\mathrm{va} / \mathrm{ms} 88287$ MSP845p 19F_single_pulse
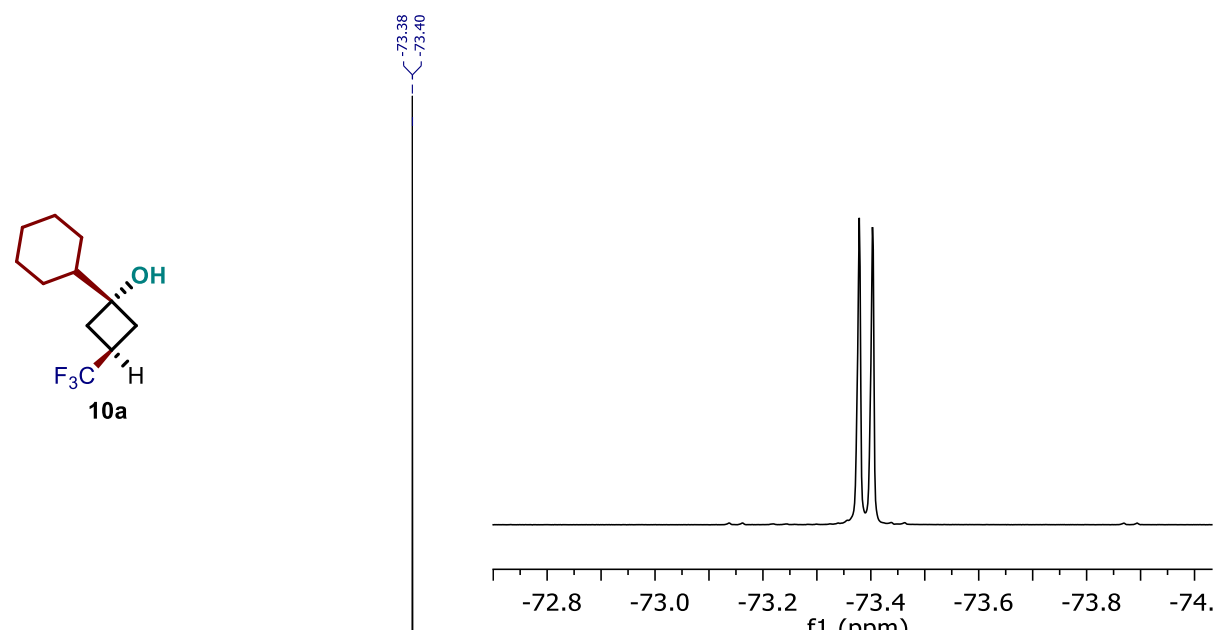

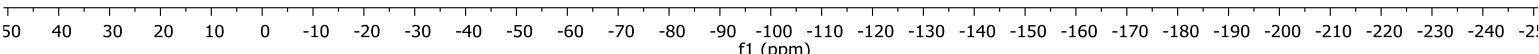


${ }^{1} \mathrm{H}$ NMR of compound $\mathbf{1 0 b}\left(500 \mathrm{MHz}, \mathrm{CD}_{3} \mathrm{CN}\right)$

ms17466_MSP847c_PROTONO1

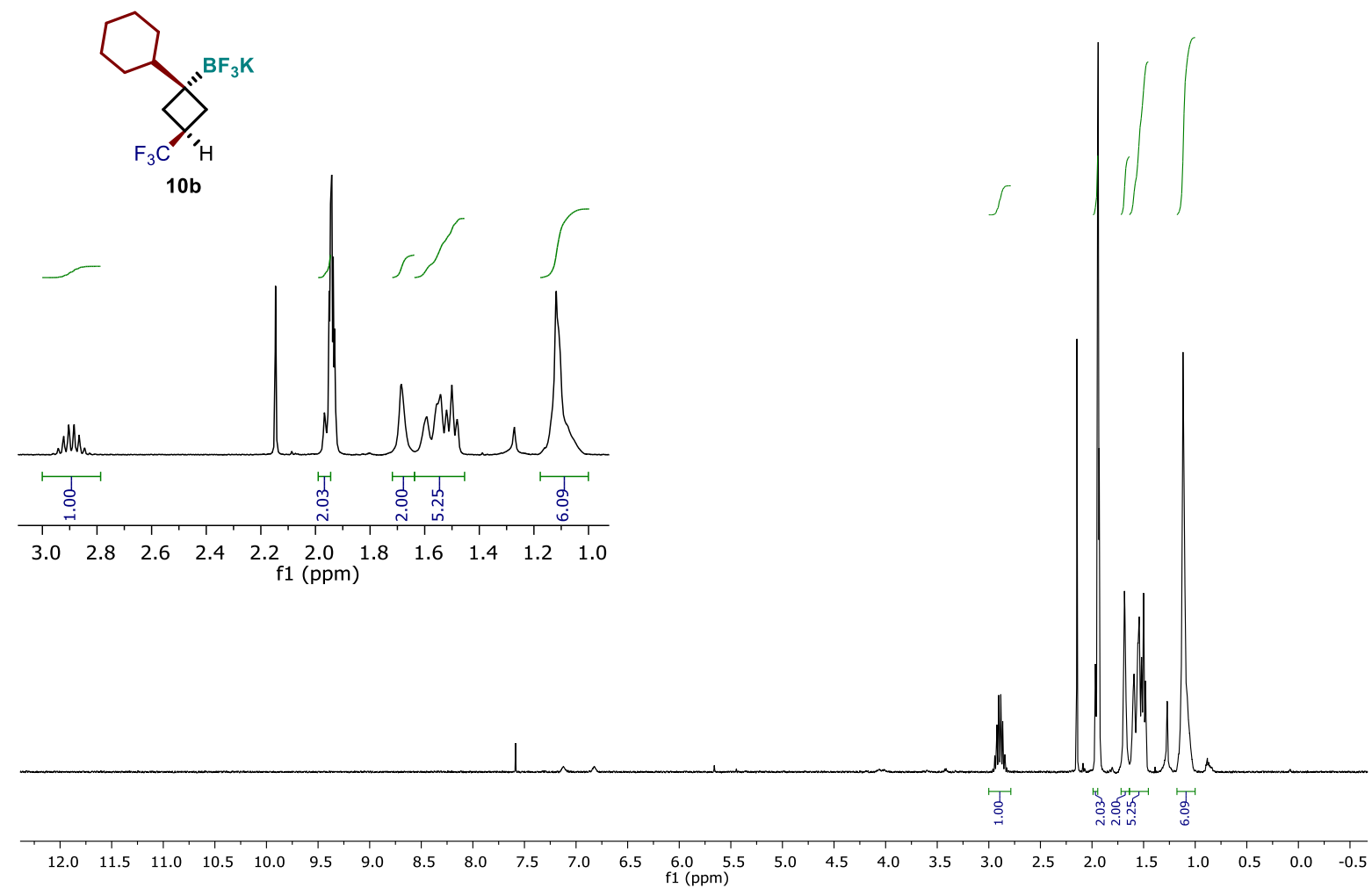

${ }^{13} \mathrm{C}$ NMR of compound $\mathbf{1 0 b}\left(125 \mathrm{MHz}, \mathrm{CD}_{3} \mathrm{CN}\right)$

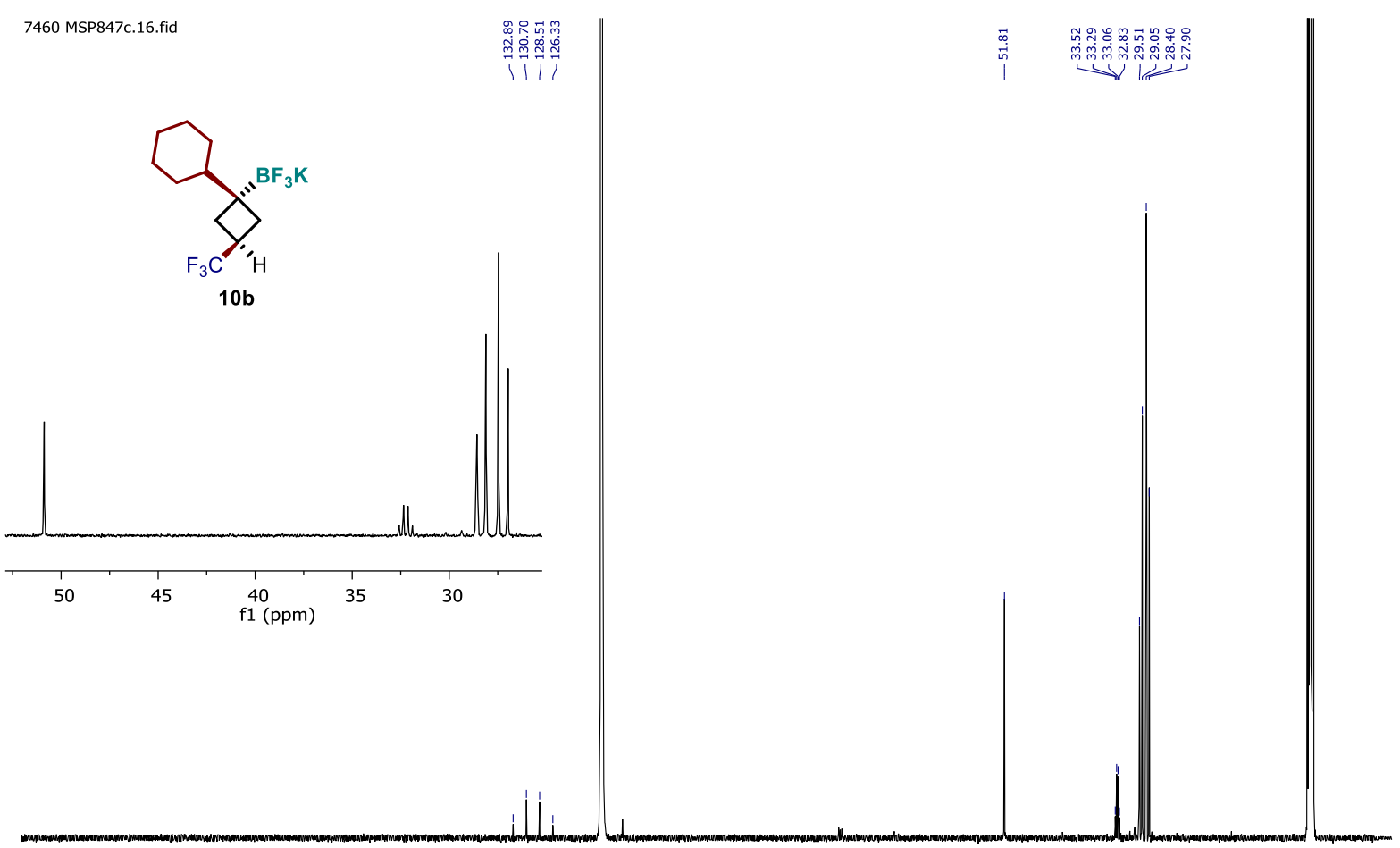

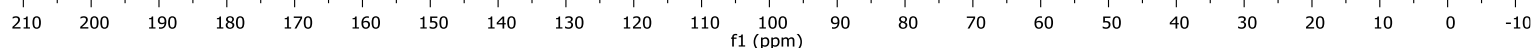


${ }^{19} \mathrm{~F}$ NMR of compound $\mathbf{1 0 b}\left(376 \mathrm{MHz}, \mathrm{CD}_{3} \mathrm{CN}\right)$

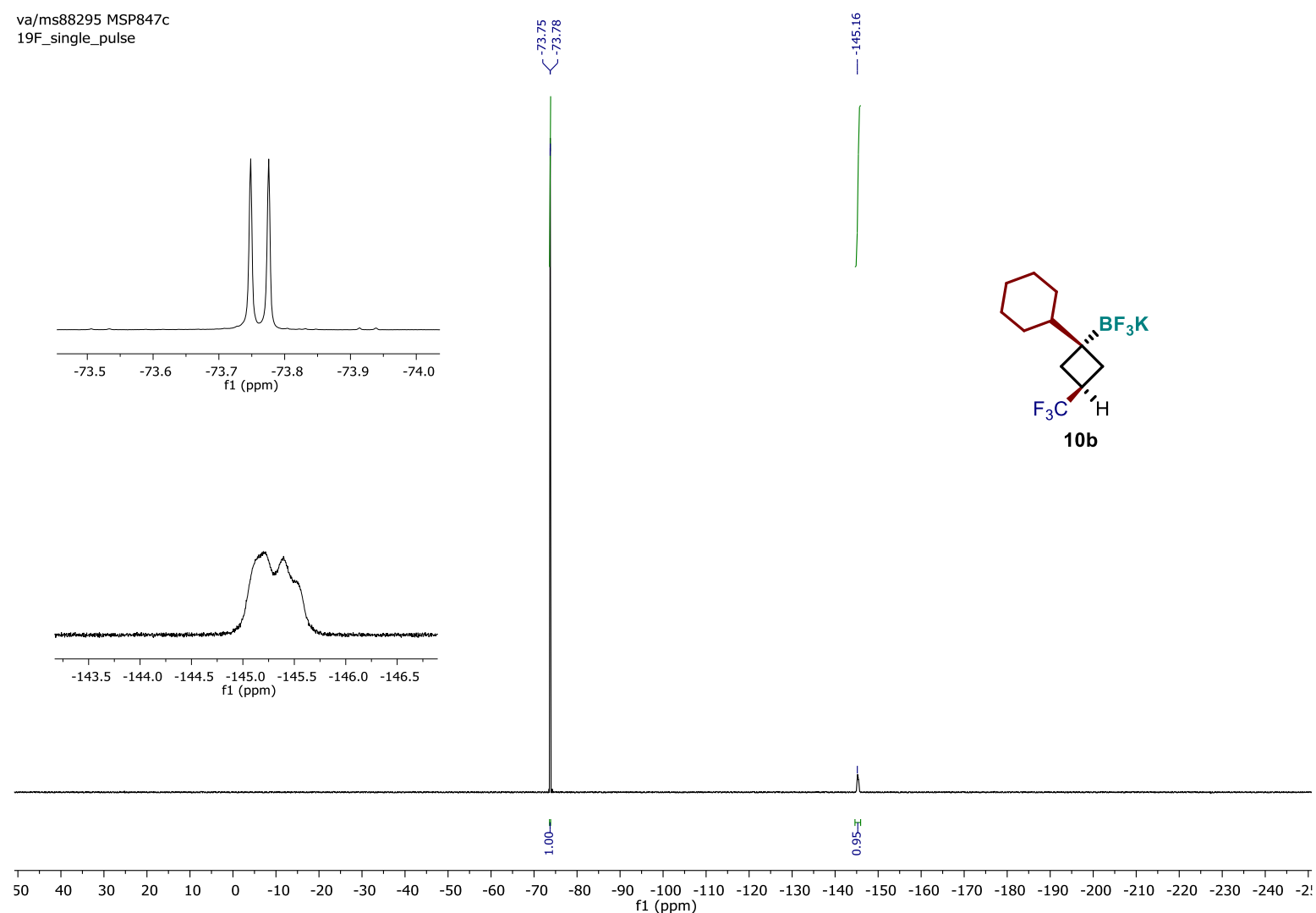

${ }^{11} \mathrm{~B}$ NMR of compound $\mathbf{1 0 b}\left(128 \mathrm{MHz}, \mathrm{CD}_{3} \mathrm{CN}\right)$
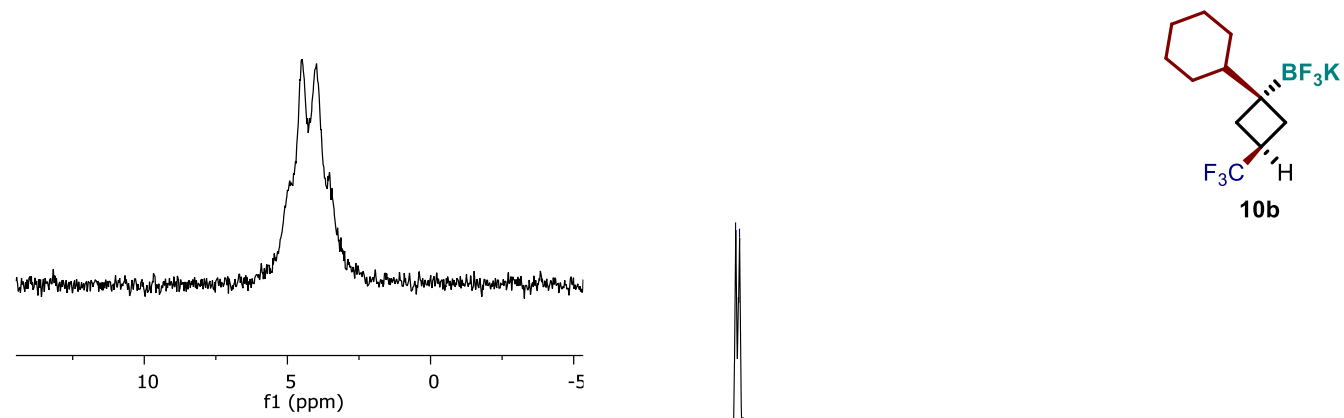

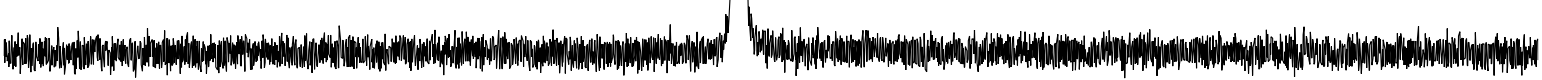

\begin{tabular}{lllllllllllllllllllllll}
\hline 00 & 90 & 80 & 70 & 60 & 50 & 40 & 30 & 20 & 10 & $\underset{\mathrm{f} 1(\mathrm{ppm})}{0}$ & -10 & -20 & -30 & -40 & -50 & -60 & -70 & -80 & -90 & -1
\end{tabular}


${ }^{1} \mathrm{H} \mathrm{NMR}$ of compound $10 \mathrm{c}\left(500 \mathrm{MHz}, \mathrm{CDCl}_{3}\right)$

ms17463_MSP846P_PROTON01

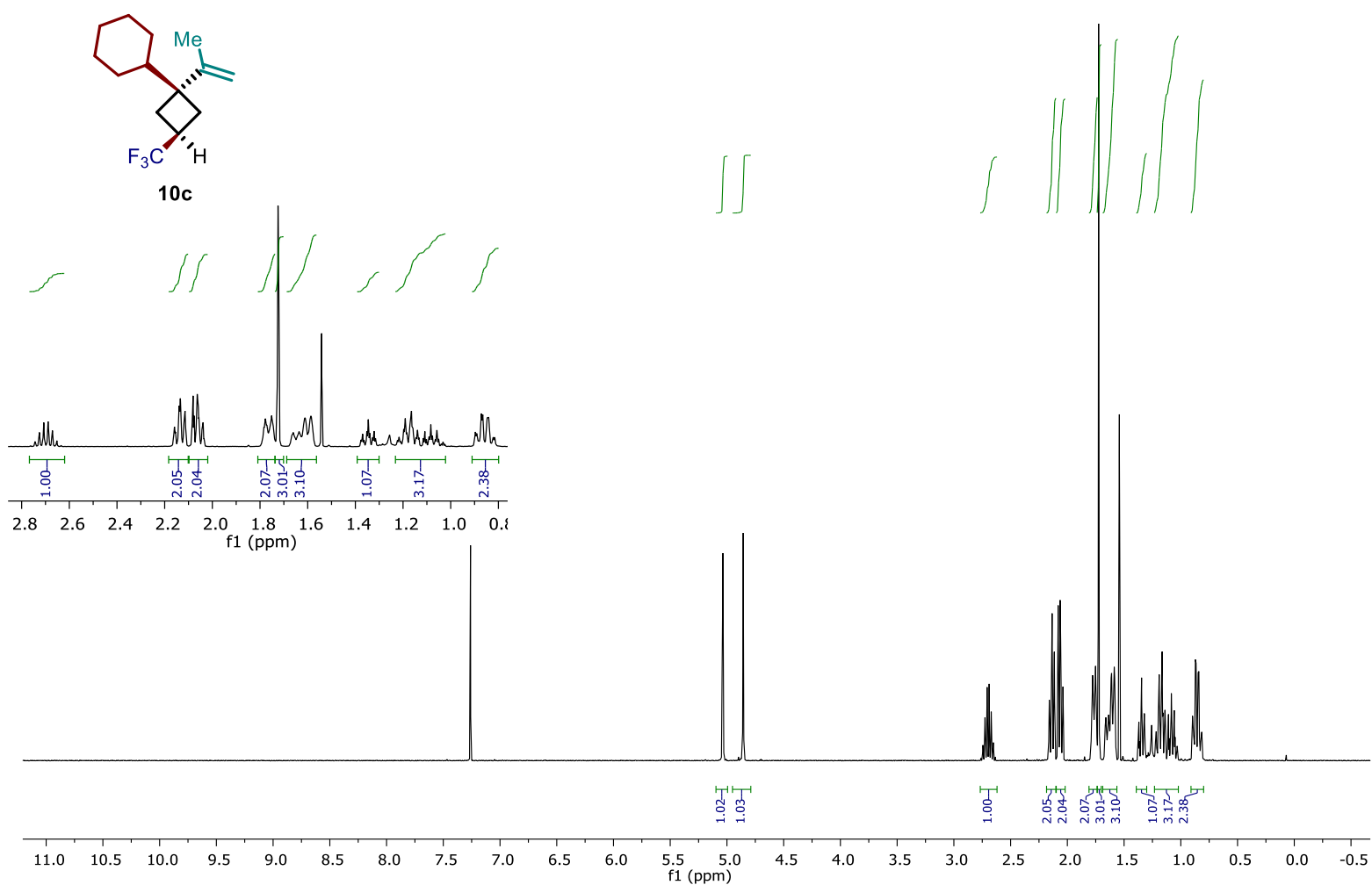

${ }^{13} \mathrm{C} \mathrm{NMR}$ of compound 10c $\left(125 \mathrm{MHz}, \mathrm{CDCl}_{3}\right)$

ms17463_MSP846P_CARBON01
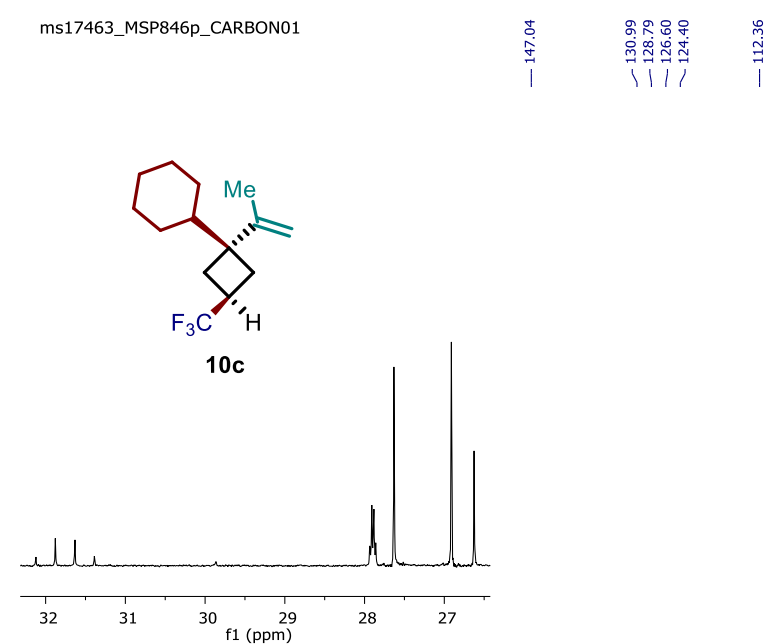

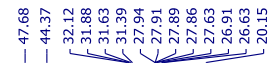

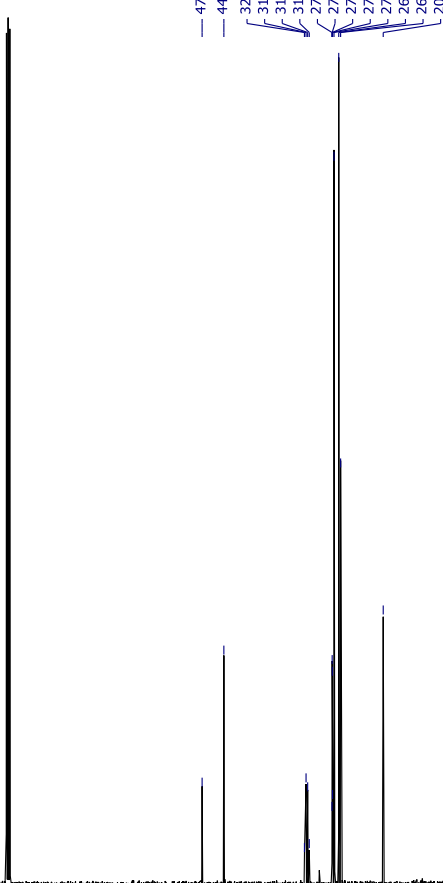

$\begin{array}{lllllllllllll}220 & 210 & 200 & 190 & 180 & 170 & 160 & 150 & 140 & 130 & 120 & 110 & 100 \\ f 1(\mathrm{ppm})\end{array}$ 
${ }^{19} \mathrm{~F}$ NMR of compound $10 \mathrm{c}\left(376 \mathrm{MHz}, \mathrm{CDCl}_{3}\right)$

va/ms88294 MSP846p_F 19F_single_pulse
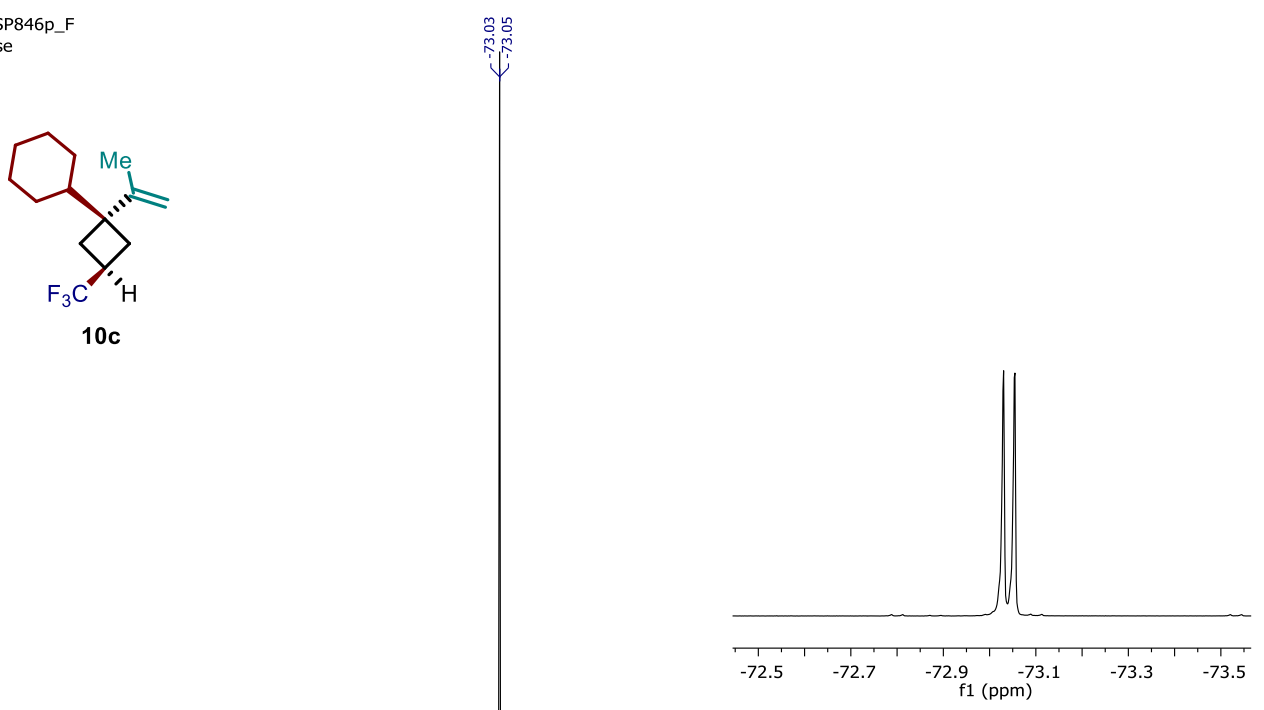

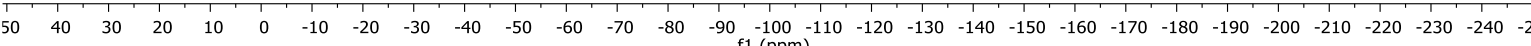


${ }^{1} \mathrm{H} \mathrm{NMR}$ of compound $\mathbf{1 0 d}\left(400 \mathrm{MHz}, \mathrm{CDCl}_{3}\right)$

48035 MSP848p.10.fid

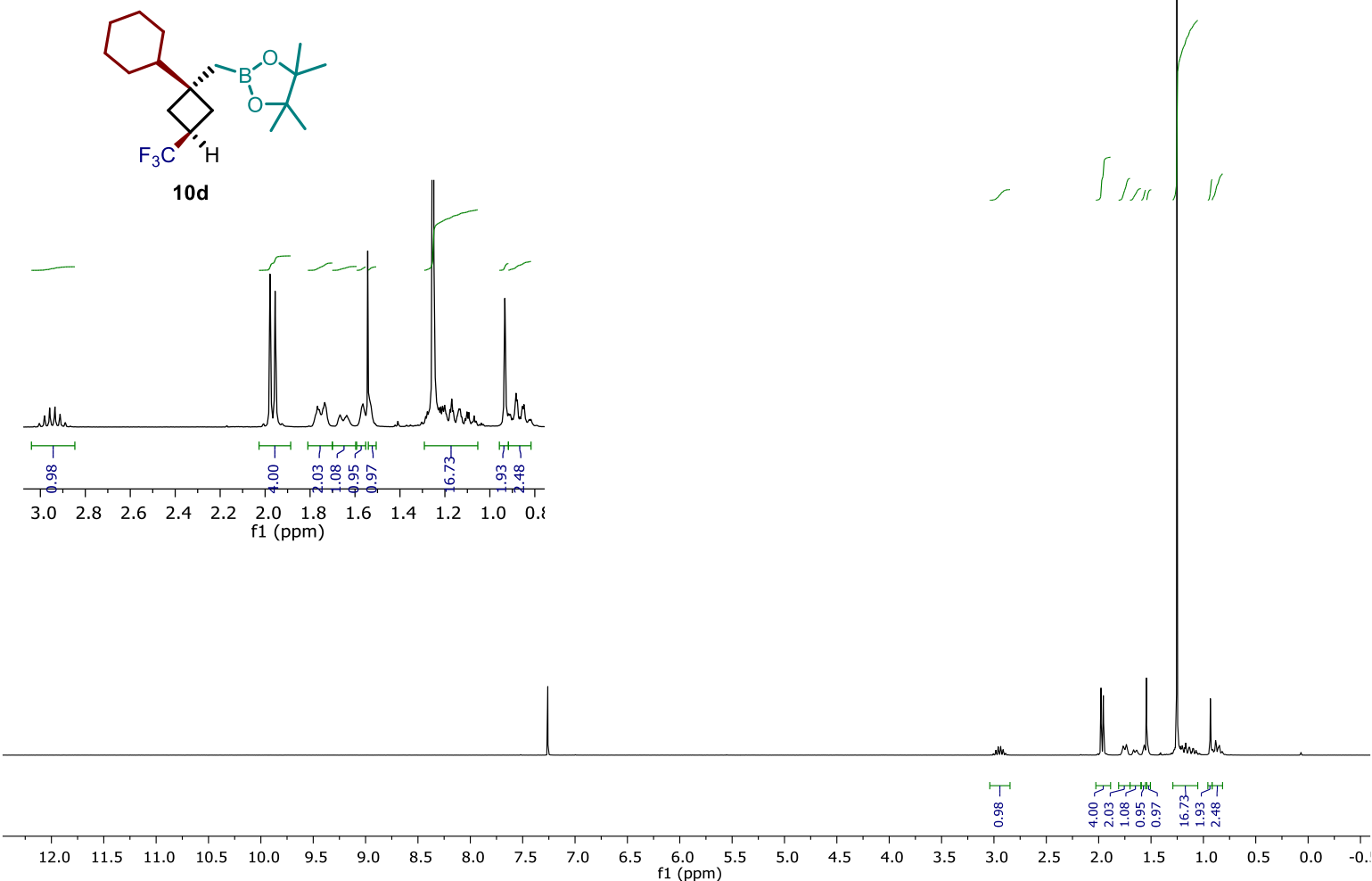

${ }^{13} \mathrm{C} \mathrm{NMR}$ of compound $\mathbf{1 0 d}\left(125 \mathrm{MHz}, \mathrm{CDCl}_{3}\right)$

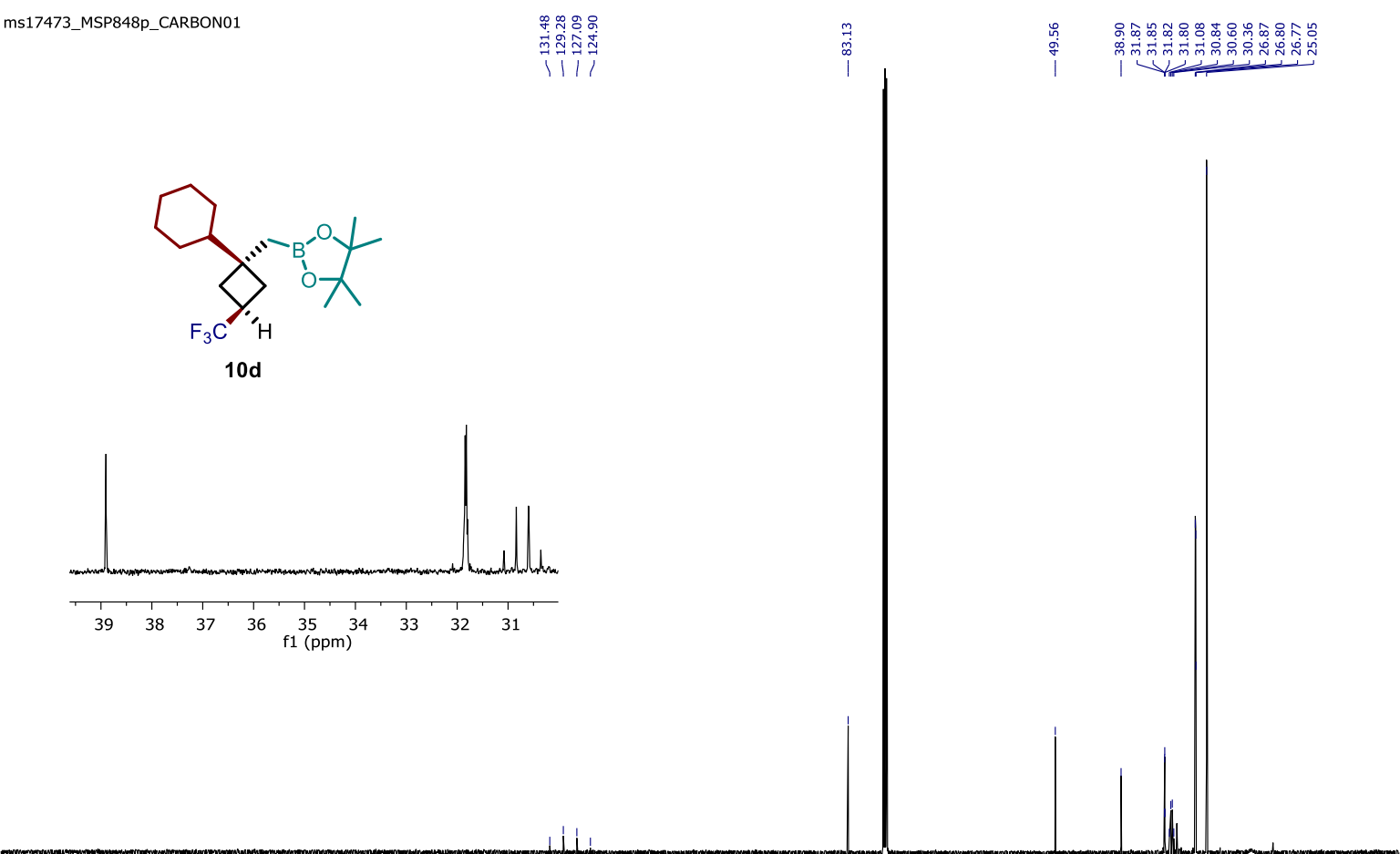

\begin{tabular}{llllllllllllllllllllllllllllll}
\hline 20 & 210 & 200 & 190 & 180 & 170 & 160 & 150 & 140 & 130 & 120 & 110 & 100 & 90 & 80 & 70 & 60 & 50 & 40 & 30 & 20 & 10 & 0
\end{tabular} 
${ }^{19} \mathrm{~F} \mathrm{NMR}$ of compound $\mathbf{1 0 d}\left(376 \mathrm{MHz}, \mathrm{CDCl}_{3}\right)$

48035 MSP848p.11.fid

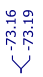
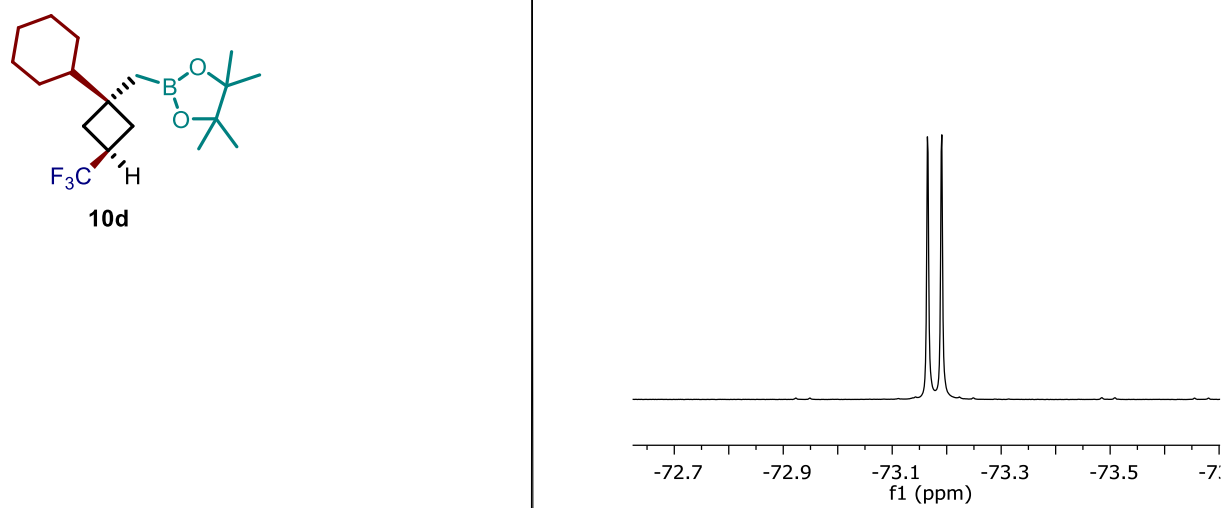

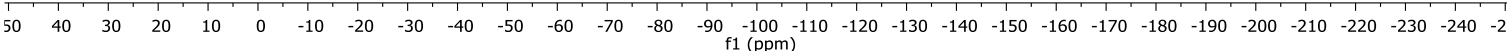

